\title{
The PIP-II Reference Design Report
}

\section{V1.00 \\ June 2015}

The PIP-II Collaboration:

Argonne National Laboratory

Brookhaven National Laboratory

Cornell University

Fermi National Accelerator Laboratory

Lawrence Berkeley National Laboratory

Michigan State University

North Carolina State University

Bhabha Atomic Research Center, Mumbai

Inter-University Accelerator Center, Delhi
Oak Ridge National Laboratory/SNS

Pacific Northwest National Laboratory

SLAC National Accelerator Laboratory

University of Tennessee

Thomas Jefferson National Accelerator

Facility

ILC/America's Regional Team

Raja Ramanna Center for Advanced

Technology, Indore

Variable Energy Cyclotron Center, Kolkota

Edited by:

Valeri Lebedev / Fermi National Accelerator Laboratory

This manuscript has been authored by Fermi Research Alliance, LLC under Contract No. DE-AC02-07CH11359 with the U.S. Department of Energy, Office of Science, Office of High Energy Physics. The U.S. Government retains and the publisher, by accepting the article for publication, acknowledges that the U.S. Government retains a non-exclusive, paid-up, irrevocable, world-wide license to publish or reproduce the published form of this manuscript, or allow others to do so, for U.S. Government purposes. 


\section{Contents}

1. PIP-II PERFORMANCE GOALS AND SUMMARY .........................................................................4

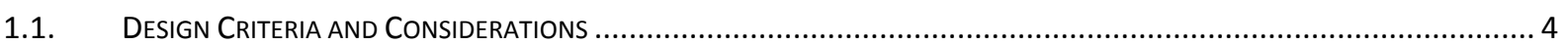

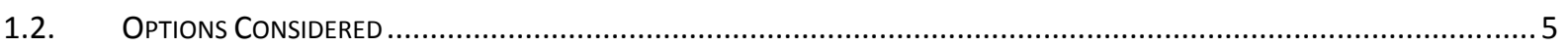

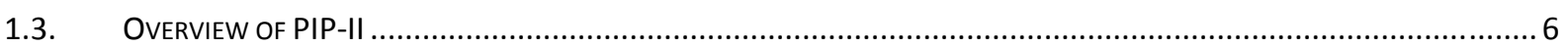

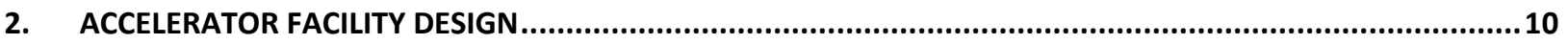

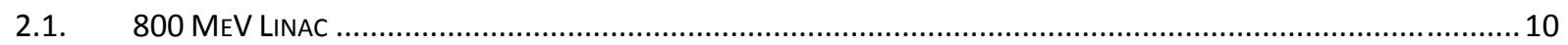

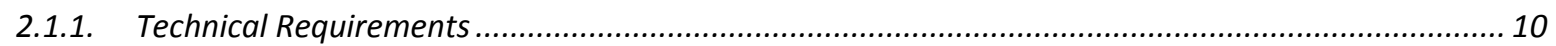

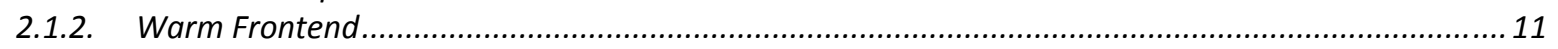

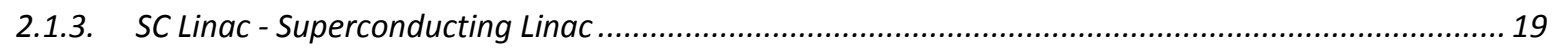

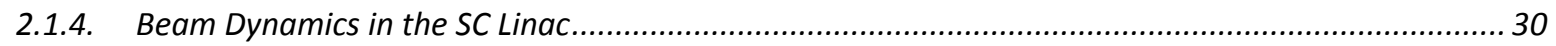

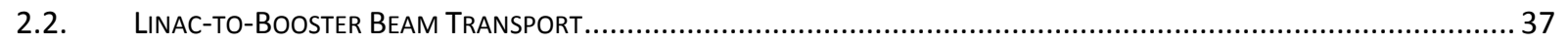

2.2.1. Particle Loss and Limitations on Beam Transport Parameters ..................................................37

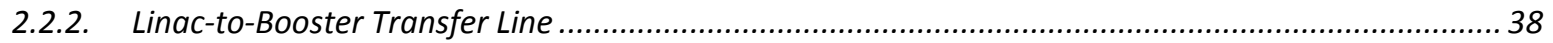

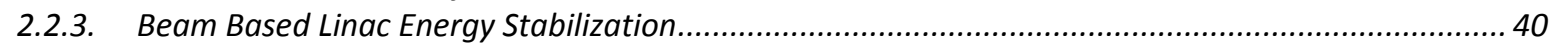

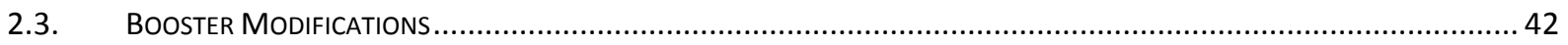

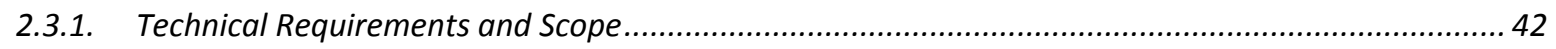

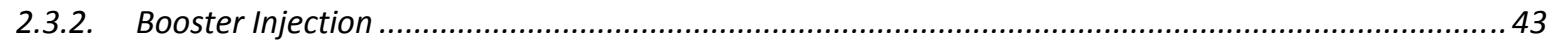

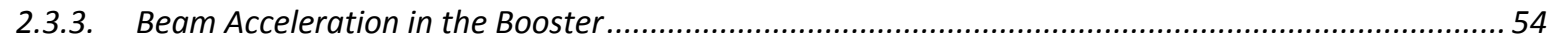

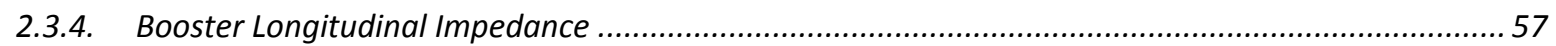

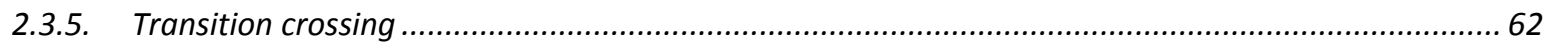

2.3.6. Modifications to the Magnet System Required for $20 \mathrm{~Hz}$ Operation ...........................................63

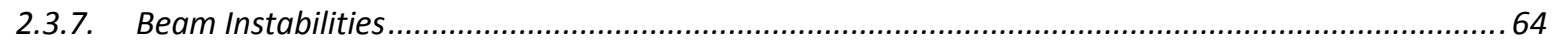

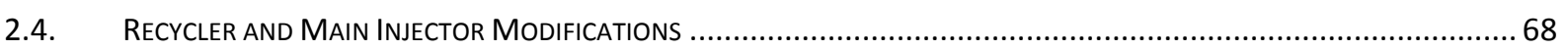

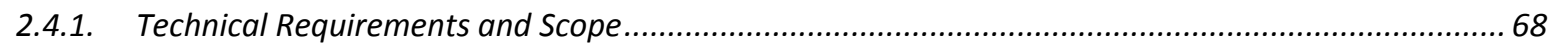

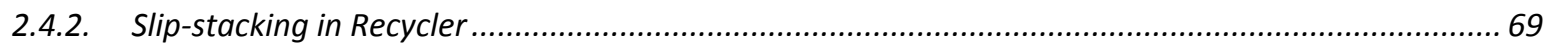

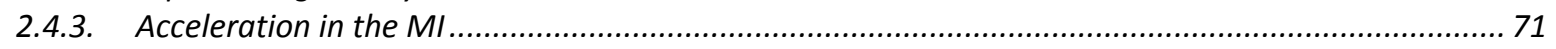

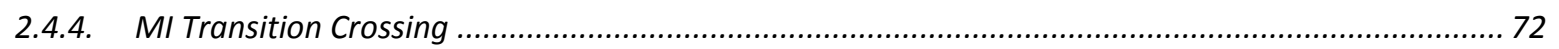

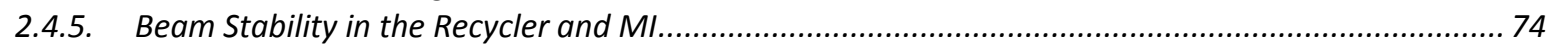

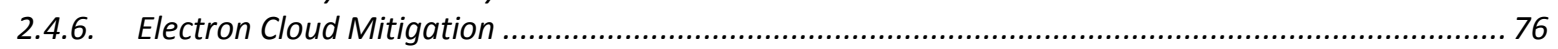

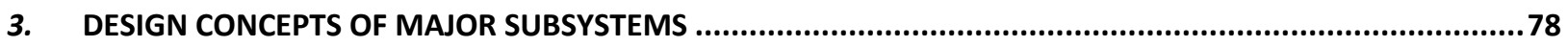

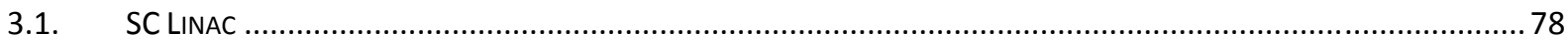

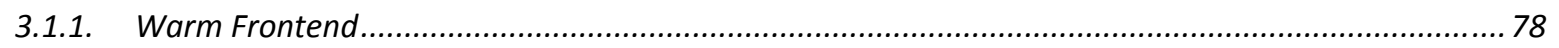

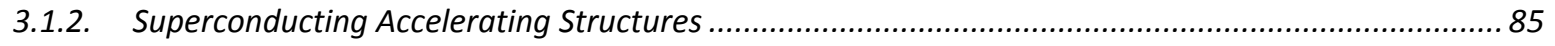

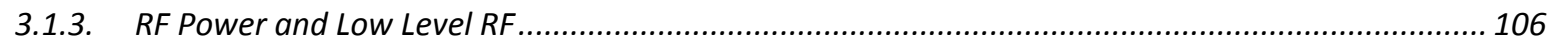

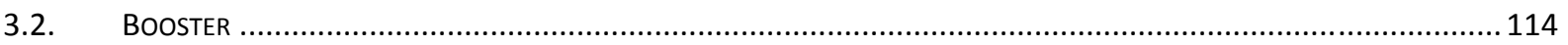

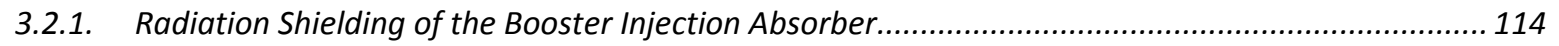

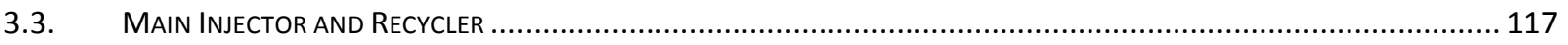

3.3.1. Hardware for Main Injector Transition Crossing ............................................................. 117

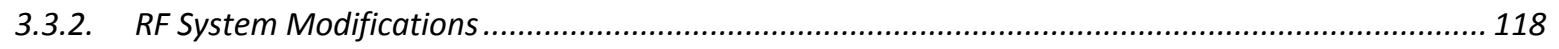

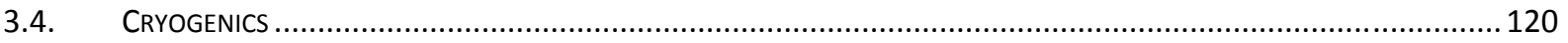

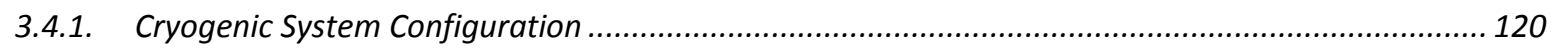

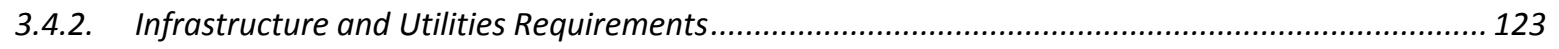

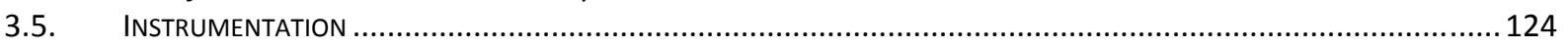

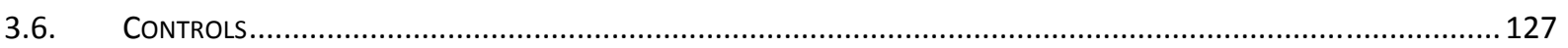

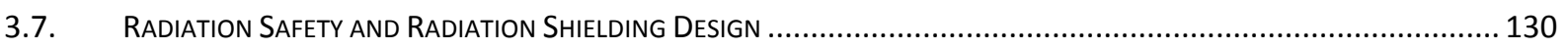

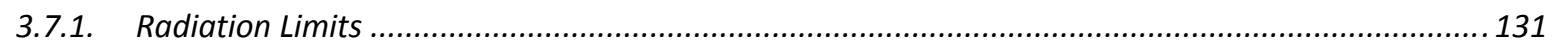

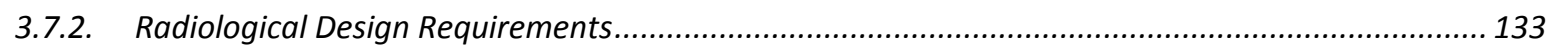

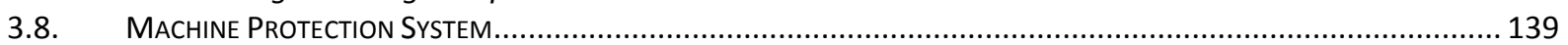


3.8.1. MPS Configuration .

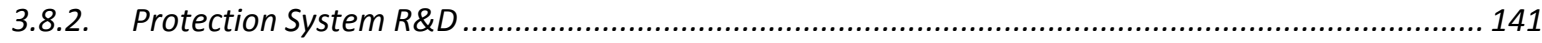

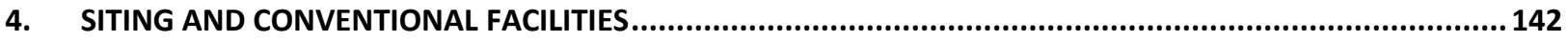

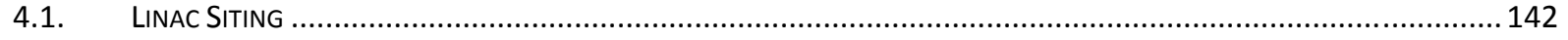

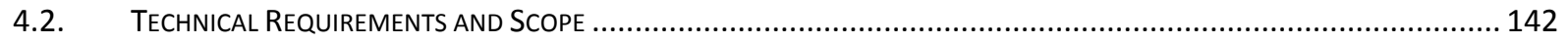

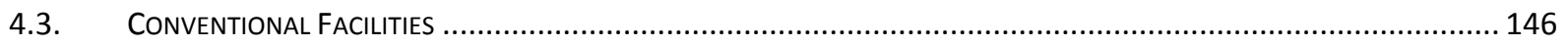

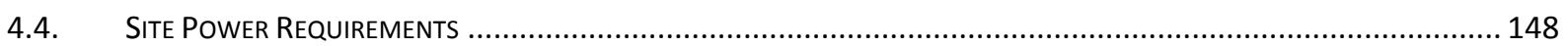

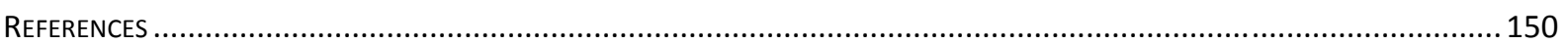




\section{PIP-II Performance Goals and Summary}

The Proton Improvement Plan-II (PIP-II) is a high-intensity proton facility being developed to support a world-leading neutrino program over the next two decades at Fermilab. PIP-II is an integral part of the U.S. Intensity Frontier Roadmap as described in the Particle Physics Project Prioritization Panel (P5) report of May 2014 [1]. As an immediate goal PIP-II is focused on upgrades to the Fermilab accelerator complex capable of providing a beam power in excess of 1 MW on target at the initiation of LBNF [1,2] operations. PIP-II is a part of a longer-term concept for a sustained campaign of upgrades and improvements to achieve multi-MW capabilities at Fermilab.

PIP-II is based on three major thrusts. They are (1) the recently completed upgrades to the Recycler and Main Injector (MI) for the NOvA experiment, (2) the Proton Improvement Plan [3] currently underway, and (3) the Project X Reference Design [4].

Note that:

- The Proton Improvement Plan (PIP) consolidates a set of improvements to the existing Linac, Booster, and Main Injector (MI) aimed at supporting $15 \mathrm{~Hz}$ Booster beam operation. In combination, the NOvA upgrades and PIP create a capability of delivering $700 \mathrm{~kW}$ beam power from the Main Injector at $120 \mathrm{GeV}$;

- The scope of the Project X Reference Design Report was aimed well beyond PIP. It described a complete concept for a multi-MW proton facility that could support a broad particle physics program based on neutrino, kaon, muon, and nucleon experiments $[5,6]$. The Project $\mathrm{X}$ conceptual design has evolved over a number of years, incorporating continuous input on physics research goals and advances in the underlying technology development programs [7,8,9]. PIP-II, to high degree, inherits these goals as the goals for future developments and upgrades.

This document (PIP-II Reference Design Report) describes an initial step in the development of the Fermilab accelerating complex. The plan described in this Report balances the far-term goals of the Laboratory's long baseline neutrino mission with the near- and mid-term goals identified at the Snowmass workshop [10] and endorsed by the P5 report [1].

\subsection{Design Criteria and Considerations}

The existing Fermilab accelerator complex could be upgraded using a number of different approaches in order to achieve beam power in excess of $1 \mathrm{MW}$ on the LBNF target. The challenge is to identify solutions that provide an appropriate balance between minimizing near-term costs and maintaining the flexibility to support longer-term physics goals. In order to constrain consideration to a modest number of options the following criteria are applied to possible solutions:

- The plan should support the delivery of at least $1 \mathrm{MW}$ of proton beam power from the Main Injector to the LBNF target at energies between $60-120 \mathrm{GeV}$;

- The plan should provide support to the currently envisioned $8 \mathrm{GeV}$ program, including the Mu2e and g-2 experiments, as well as the suite of short-baseline experiments [5,6];

- The plan should provide a platform for eventual extension of beam power to LBNF to more than $2 \mathrm{MW}$;

- The plan should include a future capability to support multiple rare processes experiments with high duty factor beams at high beam power. 
The primary bottleneck limiting beam power to the LBNF target is the existing Linac/Booster. Performance is limited to about $4.4 \times 10^{12}$ protons per Booster pulse by beam loss - primarily driven by space-charge at the $400 \mathrm{MeV}$ injection energy. The secondary potential bottleneck is slipstacking of twelve Booster pulses in the Recycler. This performance is determined jointly by characteristics of the Recycler itself and by the characteristics of beam delivered from the Booster.

The ideal facility meeting the above criteria would be a modern $8 \mathrm{GeV}$ superconducting linac for injection either into the Main Injector or Recycler as described in the Project X RDR, or the pairing of an $\sim 2 \mathrm{GeV}$ SRF linac with a modern Rapid Cycling Synchrotron. These options provide performance that would significantly exceed the first design criteria, and would meet all subsequent criteria, but also significantly exceed the likely available funding.

\subsection{Options Considered}

Two options were considered that we believe could meet the first design criterion listed above. Both options assume completion of the Proton Improvement Plan (PIP), enabling $15 \mathrm{~Hz}$ beam operations of the Booster at $4.4 \times 10^{12}$ protons per pulse. They are based on raising the injection energy of the Booster to provide a $50 \%$ increase in delivered protons per pulse. Paired with a modest decrease of the Main Injector cycle time (from 1.333 to 1.2 seconds) this provides $1.2 \mathrm{MW}$ beam power at $120 \mathrm{GeV}$. Another possibility based on proton accumulation within the existing antiproton source is not considered here because it is incompatible with lab's future plans.

\section{1. $800 \mathrm{MeV}$ Superconducting pulsed linac}

This option is a scaled down implementation of Stage 1 of the Project X Reference Design, focused on the neutrino mission. It consists of an $800 \mathrm{MeV}$ superconducting pulsed linac, injecting into the Booster, and located in an enclosure in close proximity to existing electrical, water, and cryogenic infrastructure. Compared to the Project X Reference Design, operations at low duty factor $(\sim 1 \%$ for beam pulses) and the choice of siting lead to significant cost savings. Constructing the linac from continuous wave $(\mathrm{CW})$ capable cavities and cryomodules offers a straightforward future upgrade path, at minimal additional cost, that would preserve the full breadth of scientific opportunities described in the Project X RDR. This approach meets all the design criteria listed above and is expected to be attractive to potential international partners.

\section{2. $400 \mathrm{MeV}$ "afterburner" to the existing $400 \mathrm{MeV}$ linac}

It is possible to contemplate the construction of a new superconducting pulsed linac at the end of the existing $400 \mathrm{MeV}$ pulsed linac. This implementation would require physical relocation of the existing linac, upstream by about $50 \mathrm{~m}$, to make space for the superconducting extension. The advantage of this approach is that it would cost less than option 1. The disadvantages are as follows:

a) Upgrade paths to $\mathrm{CW}$ operations are problematic because of the extended room temperature section;

b) The linac frequency $(805 \mathrm{MHz})$ is not consistent with the significant $\mathrm{R} \& \mathrm{D}$ investment already made at $650 \mathrm{MHz}$;

c) A significant contribution from our international partners would probably not be possible due to items a) and b);

d) Vulnerabilities would remain in the existing linac. In particular, the drift tube linac portion currently relies on RF sources obtainable only from a single vendor and for which there is a minimal market demand; and 
e) This approach would require a significant interruption to the operating program ( $\sim 1$ year) for relocation and installation.

Option 1 is preferred, and will be described in this report, because it provides the most robust accelerator complex in support of the neutrino programs, and because it offers straightforward and cost-effective extensions to the multi-MW, high duty factor, capabilities required to support a world-leading research program based on intense beams in the longer term. This approach also minimizes disruption to the ongoing operating program, removes inherent reliability risks in linac operations, and directly capitalizes on a large amount of conceptual and technological development undertaken as part of the Project X, ILC, and LCLS-II programs. Because this option represents a natural continuation of the performance improvements being implemented within the PIP, it has been named Proton Improvement Plan-II (PIP-II).

\subsection{Overview of PIP-II}

The goal of Proton Improvement Plan-II is to enhance the capabilities of the existing accelerator complex at Fermilab to support delivery of $1.2 \mathrm{MW}$ beam power to the LBNF production target, while simultaneously providing a platform for subsequent upgrades of the accelerator complex to multi-MW capability. High-level goals, and supporting beam performance parameters, for PIP-II and their comparison to PIP parameters are given in Table 1-1. The central element of PIP-II is a new $800 \mathrm{MeV}$ superconducting linac accelerating $\mathrm{H}^{-}$ions and located in close proximity to the existing Booster as shown in Figure 1-1. This siting offers several advantages in terms of minimizing cost while retaining options for future development; in particular, the site affords direct access to significant electrical, water, and cryogenic infrastructure.

The scope encompassed by the PIP-II and described in this document includes:

- An $800 \mathrm{MeV}$ superconducting linac (SC Linac), constructed of CW-capable accelerating structures and cryomodules, operating with a peak current of $2 \mathrm{~mA}$ and a beam duty factor of $1.1 \%$;

- Beam transport from the end of the SC Linac to the new Booster injection point, and to a new $800 \mathrm{MeV}$ beam dump;

- Upgrades to the Booster to accommodate $800 \mathrm{MeV}$ injection, and acceleration of $6.5 \times 10^{12}$ protons per pulse;

- Upgrades to the Recycler to accommodate slip-stacking of $7.7 \times 10^{13}$ protons delivered by twelve Booster batches;

- Upgrades to the Main Injector to accommodate acceleration of $7.6 \times 10^{13}$ protons per pulse to $120 \mathrm{GeV}$ with a 1.2 second cycle time, and to $60 \mathrm{GeV}$ with a 0.7 second cycle time.

The linac energy is selected to support a 50\% increase in Booster beam intensity, accompanied by a $30 \%$ reduction in the space-charge tune shift as compared to the current operations. This choice is conservative and will ensure lower fractional beam loss required at the higher operating intensities and higher injection energy. The linac is constructed nearly entirely of components that are capable of operating in $\mathrm{CW}$ mode with the cryogenic system being the primary exception ${ }^{1}$. The incremental cost in constructing the linac from $\mathrm{CW}$ compatible components is minimal.

The linac is followed by a beam transport line to bring the beam to the Booster. The line includes an arc bending the beam by about $210^{\circ}$. The bending radius of the arc is maintained above

\footnotetext{
${ }^{1}$ This choice is based on existing Tevatron cryogenic infrastructure resulting in significant cost reduction. CW operation will require a new cryogenic plant.
} 
$23 \mathrm{~m}$ to prevent stripping of the $\mathrm{H}^{-}$beam prior to Booster injection. There is a provision for installation of an RF separator and septum at the linac end required to support the operation of multiple experiments following future linac upgrades.

The Booster repetition rate will be increased from 15 to $20 \mathrm{~Hz}$. It is extremely helpful for reduction of beam loss during slip-stacking in the Recycler due to the larger momentum difference between the two streams of slip-stacked bunches. The repetition rate increase also increases overall particle flux through the Booster and yields higher power for the $8 \mathrm{GeV}$ experimental program. The operation of Booster dipoles at $20 \mathrm{~Hz}$ was recently verified [11]. The transition to the higher rate will be achieved by decreasing the value of capacitors in the dipole resonance circuit. Although the acceleration rate increases proportionally to the repetition rate the required peak RF voltage stays approximately the same due to the smaller slip-factor at the injection. Doubling the RF power transferred to the beam will require minor modifications for the RF power amplifiers.

Table 1-1: PIP-II high level performance goals

\begin{tabular}{llll}
\hline \hline Performance Parameter & PIP & PIP-II & Unit \\
\hline Linac Beam Energy & 400 & 800 & $\mathrm{MeV}$ \\
Linac Beam Current & 25 & 2 & $\mathrm{~mA}$ \\
Linac Pulse Length & 0.03 & 0.55 & $\mathrm{~ms}$ \\
Linac Pulse Repetition Rate & 15 & 20 & $\mathrm{~Hz}$ \\
Linac Upgrade Potential & $\mathrm{N} / \mathrm{A}$ & $\mathrm{CW}$ & \\
Booster Protons per Pulse (extracted) & 4.2 & 6.5 & $10^{12}$ \\
Booster Pulse Repetition Rate & 15 & 20 & $\mathrm{~Hz}$ \\
Booster Beam Power @ 8 GeV & 80 & 160 & $\mathrm{~kW}$ \\
8 GeV Beam Power to LBNF & N/A & $80-120^{*}$ & $\mathrm{~kW}$ \\
Beam Power to 8 GeV Program & 30 & $80-40^{*}$ & $\mathrm{~kW}$ \\
Main Injector Protons per Pulse (extracted) & 4.9 & 7.6 & $10^{13}$ \\
Main Injector Cycle Time @ $120 \mathrm{GeV}$ & 1.33 & 1.2 & $\mathrm{sec}$ \\
Main Injector Cycle Time @ 60 GeV & N/A & 0.7 & $\mathrm{sec}$ \\
Beam Power @ 60 GeV & N/A & 1 & $\mathrm{MW}$ \\
Beam Power @ 120 GeV & $0.7^{\star}$ & $1.2^{\star}$ & $\mathrm{MW}$ \\
Upgrade Potential @ 80-120 GeV & N/A & 2.4 & $\mathrm{MW}$ \\
\hline \hline
\end{tabular}

*First number refers to Main Injector operations at $120 \mathrm{GeV}$; second number to $80 \mathrm{GeV}$.

- Applicable to $120 \mathrm{GeV}$ operation only.

• Beam power grows approximately linear for energy change from 60 to $120 \mathrm{GeV}$.

The beam is injected into the Booster using multi-turn strip-injection similar to the injection method used in the SNS [12]. The number of injection turns is equal to 300. Although the number of injection turns is much larger than what is presently used in Booster, it is still about three times less than that used in the SNS and is well within the presently used range of parameters. Large number of injection turns and small emittances of the SC Linac beam allow painting of transverse and longitudinal distributions resulting in a significant reduction of space-charge effects. In contrast to present operations, beam injection will proceed at non-zero RF voltage. This allows one 
to avoid adiabatic bunching, which would be problematic because of the long bunching time ${ }^{2}$. To reduce beam loss during Booster injection the linac bunches arriving at the RF bucket boundaries are removed by a bunch-by-bunch chopper located in the Medium Energy Beam Transport (MEBT) of the linac. The same chopper creates a three bunch long extraction gap. Slip-stacking in the Recycler and acceleration in the MI will be done in a manner similar to that presently used for NOvA [13].

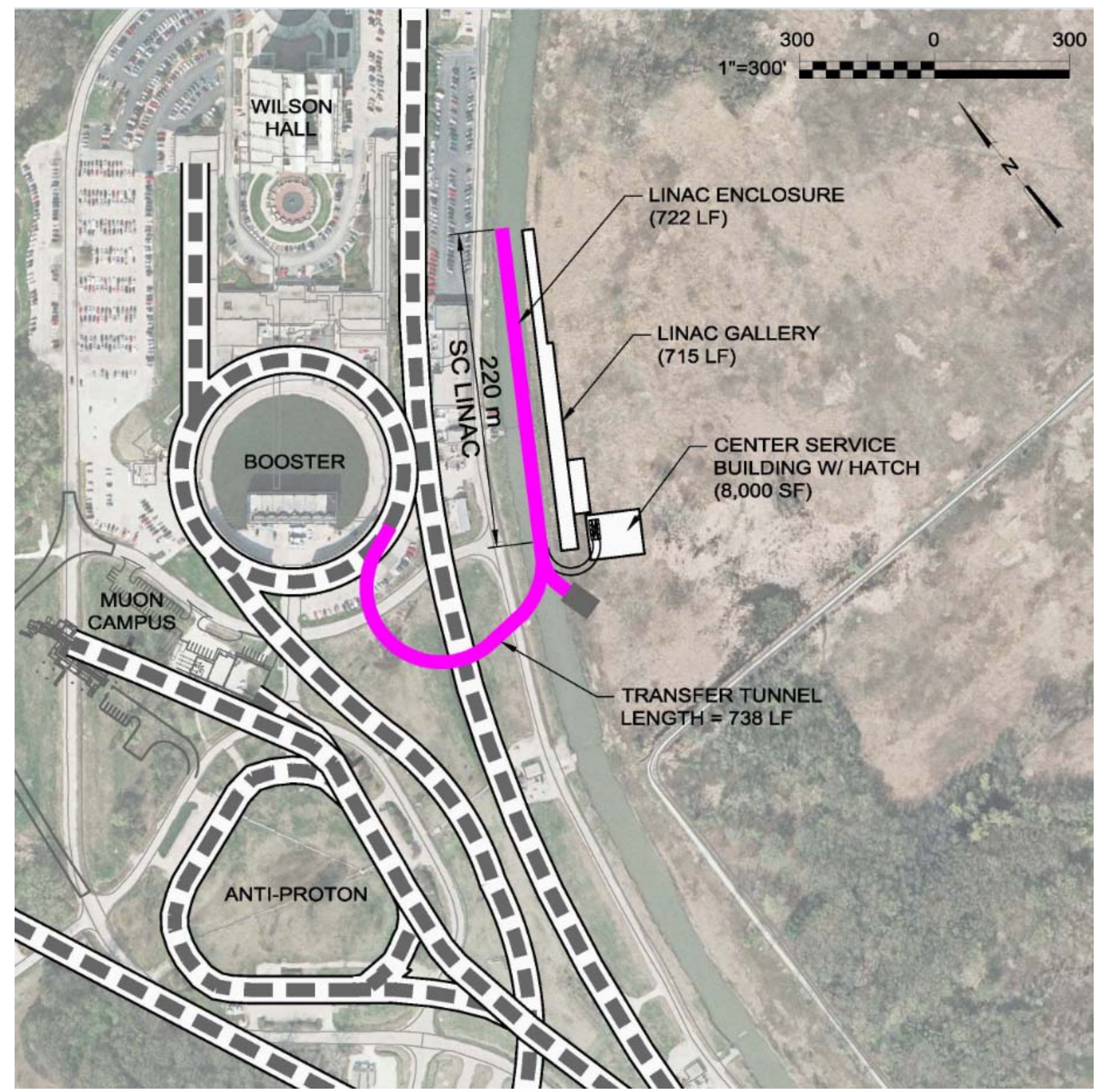

Figure 1.1: Site layout of PIP- II. New construction includes the linac enclosure, transfer line enclosure, linac gallery, center service building, utility corridor, and cryo building. Grey dashed areas represent existing underground enclosures.

\footnotetext{
${ }^{2}$ The long bunching time is related to the smaller value of the slip-factor at the higher injection energy. The higher Booster repetition rate additionally magnifies this problem.
} 
Upgrades to a number of systems in the Booster, Recycler, and Main Injector will be required in order to support the higher Booster injection energy and higher beam intensities. These include upgrades to the Booster injection system, the RF systems in all rings, and various feedback systems. The upgrade to the Booster injection system is the most significant of these.

Modifications to the LBNF target facility to accept $1.2 \mathrm{MW}$ protons are assumed to be undertaken by the LBNF project. However, requirements for the beam delivery are described in this document. Note that the concept presented here is capable of delivering from 1 to $1.2 \mathrm{MW}$ of beam power to LBNF for the energy change from 60 to $120 \mathrm{GeV}$. Although the LBNF operation is expected to take a major fraction of Booster intensity, considerable power will still be available at 8 $\mathrm{GeV}$. In particular, it is expected to be $82 \mathrm{~kW}$ for $120 \mathrm{GeV}$ operations and $23 \mathrm{~kW}$ for $60 \mathrm{GeV}$.

PIP-II provides a variety of straightforward and cost effective upgrade paths. Delivery of more than $2 \mathrm{MW}$ to the LBNF target will require replacement of the existing Booster. The most effective strategy would be to extend the $0.8 \mathrm{GeV}$ linac to $8 \mathrm{GeV}$ and inject directly into the Main Injector at the MI-10 straight section. This linac would be based on the superconducting technologies developed for PIP-II, and would have significant technological overlap with the $1.3 \mathrm{GHz}$ cryomodules Fermilab will be supplying to the LCLS-II Project [14] over the next five years. Alternatively, the linac could be extended to $2-3 \mathrm{GeV}$, followed by an RCS. Upgrading the linac to $\mathrm{CW}$ operations is achievable by upgrading the PIP-II cryogenic system. CW operations of the linac could support MW-class beam delivery to a variety of rare processes experiments, including Mu2e.

The estimated cost of PIP-II is $\sim \$ 600 \mathrm{M}$ in 2020 dollars, including both development and construction costs, related accelerator improvement projects (AIPs), direct and indirect costs, and $40 \%$ contingency. Potential offsets to this number in the form of possible international in-kind contributions are valued at $\sim \$ 150 \mathrm{M}$.

It is worth noting that while the configuration described here is cost-effective, no system-wide (Linac, Booster, Recycler, Main Injector) cost optimization has been completed at this time. It is anticipated that such an optimization will become possible once more details emerge with further design work. 


\section{Accelerator Facility Design}

It is envisioned that the PIP-II construction project will include only the superconducting linac (SC Linac) and the transfer line connecting the linac and the Booster. However, the upgrades to the Booster, Main injector and Recycler are an integral part of the plan and therefore are described below in the same detail as the linac.

\section{1. $800 \mathrm{MeV}$ Linac}

\subsubsection{Technical Requirements}

The linac includes the following major elements:

- Ion source,

- Low Energy Beam Transport (LEBT),

- RFQ,

- Medium Energy Beam Transport (MEBT), including the chopper and bunching cavities,

- One accelerating section composed of 162.5 MHz Half-Wave Resonators (HWR),

- two accelerating sections composed of $325 \mathrm{MHz}$ Single-Spoke Resonators (SSR1 and SSR2),

- Two accelerating sections of $650 \mathrm{MHz}$ elliptical cavities, one at low beta (0.647) and one at high beta (0.971) (LB650 and HB650).

Figure 2.1 shows the structure of the linac. A room temperature (RT) section accelerates the beam to $2.1 \mathrm{MeV}$ and creates the desired bunch structure for injection into the SC Linac. The RFQ and the first $\mathrm{SC}$ section (HWR) operate in the $\mathrm{CW}$ mode. To reduce the required cryogenic power the other accelerating structures operate in the pulsed mode. However they are designed and built to be $\mathrm{CW}$ compatible in order to accommodate future upgrades. Operation with a peak current of up to $10 \mathrm{~mA}$ is supported by the ion source, LEBT and RFQ. The bunch-by-bunch chopper located in the MEBT removes undesired bunches leaving the beam current at up to $2 \mathrm{~mA}$ (averaged over a few $\mu \mathrm{s}$ ) for further acceleration. There is also a "slow" chopper in the LEBT with rise and fall times of about $100 \mathrm{~ns}$. It allows forming a macro-structure in the beam timing required for machine commissioning and allows one to avoid unnecessary beam loading in normal operations. Together the LEBT and MEBT choppers form the desired bunch structure.

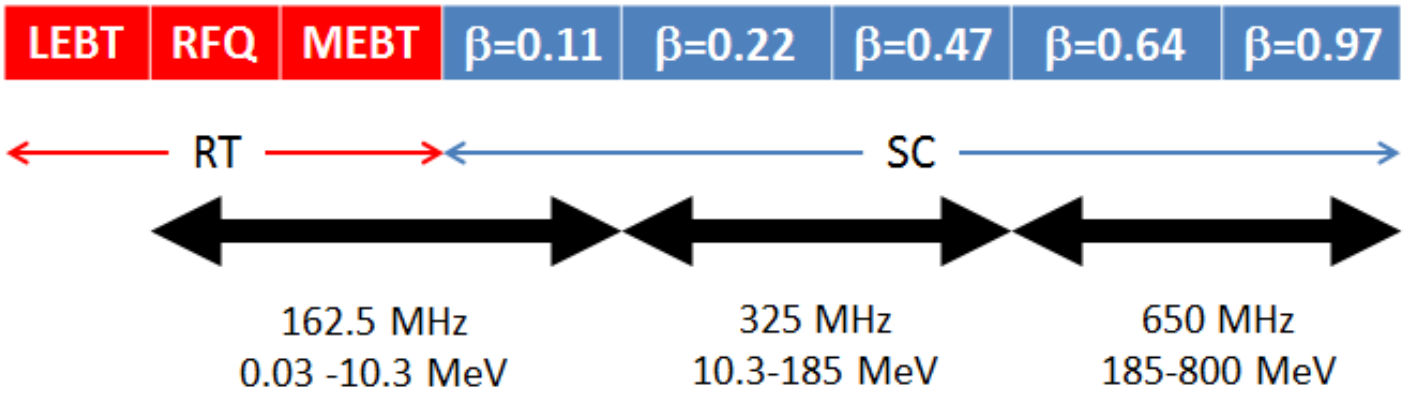

Figure 2.1: The linac technology map.

The energy stored in the SC cavities is quite large. Consequently, the accelerating voltage fluctuations due to beam loading are below $10^{-3}$ if the bunch structure is repetitive with period below about $3 \mu \mathrm{s}$. The SC Linac accelerates to $800 \mathrm{MeV}$ up to $2 \mathrm{~mA}$ of beam current with peak 
currents of up to $10 \mathrm{~mA}$ for periods of less than a few $\mu \mathrm{s}$. The operational parameters for the SC Linac are given in Table 2.1.

Table 2.1: SC Linac Parameters

\begin{tabular}{l|l|l}
\hline Parameter & Requirement & \\
\hline Particle species & $\mathrm{H}^{-}$ & \\
\hline Input beam energy (Kinetic) & 2.1 & $\mathrm{MeV}$ \\
\hline Output beam energy (Kinetic) & 0.8 & $\mathrm{GeV}$ \\
\hline Pulse repetition rate & 162.5 & $\mathrm{MHz}$ \\
\hline RF pulse length & pulsed-to-CW & \\
\hline Sequence of bunch pulses & Programmable & \\
\hline Average beam current in SC Linac & 2 & $\mathrm{~mA}$ \\
\hline Final rms norm. transverse emittance, $\varepsilon_{x}=\varepsilon_{y}$ & $<0.3$ & $\mathrm{~mm}-\mathrm{mrad}$ \\
\hline Final rms norm. longitudinal emittance & $<0.35(1.1)$ & $\mathrm{mm}-\mathrm{mrad}(\mathrm{keV}-\mathrm{ns})$ \\
\hline Rms bunch length at the SC Linac end & 4 & $\mathrm{ps}$ \\
\hline
\end{tabular}

To support beam injection into the Booster, pulsed operation of the linac is sufficient. In this case the linac operates at $20 \mathrm{~Hz}$ with a beam pulse duration of $0.55 \mathrm{~ms}$ resulting in $1.1 \%$ beam duty factor. RF cavity filling requires a significantly longer time. The effective cryogenic duty factor is about $6.6 \%$ while the effective duty factor for high power RF is about $15 \%$. To reduce the cryogenic power the phase of the RF amplifiers can be shifted by $180 \mathrm{deg}$. after a beam pulse to accelerate voltage decay in cavities.

Maintaining sufficiently small emittances through the entire linear accelerator and the beam transport to the Booster is essential for minimizing the beam loss both in the linac and at an injection to the Booster. The maximum allowed rms emittances for the ion source beam current in the range of $2 \div 10 \mathrm{~mA}$ are presented in Table 2.2 .

Table 2.2: Maximum allowed rms normalized emittances through the accelerator

\begin{tabular}{l|c|c}
\hline \multirow{2}{*}{} & \multicolumn{2}{|c}{ Normalized rms beam emittance (mm mrad) } \\
\cline { 2 - 3 } & Transverse & Longitudinal \\
\hline Ion source & 0.14 & - \\
\hline RFQ entrance & 0.18 & - \\
\hline RFQ exit & 0.20 & 0.28 \\
\hline MEBT exit & 0.23 & 0.31 \\
\hline Exit of SC linac & 0.3 & 0.35 \\
\hline
\end{tabular}

\subsubsection{Warm Frontend}

The warm frontend of the PIP-II linac provides an $\mathrm{H}^{-}$beam to the first superconducting module. The frontend beam current specifications go well beyond what is required for PIP-II operation in order to enable high proton flux experiments without significant future upgrades. While the 
nominal PIP-II peak current for the Booster injection is about $4 \mathrm{~mA}$, the nominal average current of the RFQ is $5 \mathrm{~mA}$ (10 mA maximum). The frontend design incorporates a fast chopper to provide bunch-by-bunch selection for in-bucket injection into the Booster. This capability will also be used in future multi-user operation modes.

The frontend consists of a $30 \mathrm{keV} \mathrm{H}^{-}$ion source, a Low Energy Beam Transport (LEBT) delivering up to $10 \mathrm{~mA}$ DC beam to the entrance of a $2.1 \mathrm{MeV} \mathrm{CW}$ Radio Frequency Quadrupole (RFQ) accelerator, and a Medium Energy Beam Transport (MEBT). This is shown schematically in Figure 2.1.

The choice for the LEBT energy of $30 \mathrm{keV}$ is a compromise between considerations of beam space charge effects that may increase the transverse emittance at low energy and RFQ adiabatic bunching, where the longitudinal emittance improves with decreasing the injection energy. This choice balances the final warm frontend emittance among the three degrees of freedom.

The RFQ energy of $2.1 \mathrm{MeV}$ is chosen because it is below the neutron production threshold for most materials, thereby simplifying the RFQ and MEBT maintenance. At the same time, this energy is sufficiently large to mitigate space charge effects in the MEBT at currents as high as 10 mA.

\subsubsection{LEBT - Low Energy Beam Transport}

The layout of the ion source and LEBT is shown in Figure 2.2. Two ion sources are installed to maximize the beam availability. Each source can be removed for repairs, reinstalled, and conditioned without interrupting the operation of the other source. The LEBT transports the beam from the exit of the ion source to the entrance of the RFQ. It also matches the beam envelopes at its end to the ones required for low loss acceleration in the RFQ. In addition, the LEBT forms a lowduty factor beam during commissioning and tuning of the downstream beam line in a pulsed mode. It also interrupts the beam as part of the machine protection system (MPS), and prohibits beam generation accordingly to the safety system status.

The LEBT Functional Requirement Specifications (FRS) are listed in [15]. The LEBT includes 3 solenoids (for each leg), a slow switching dipole magnet, a chopper assembly, and diagnostics to characterize and tune the beam. The $x$ and $y$ dipole correctors are mounted inside each solenoid. The edge focusing of the switching dipole is adjusted to minimize the asymmetry between horizontal and vertical focusing. The $\sim 2 \mathrm{~m}$ beam line length ensures that the gas migration from the ion source to the RFQ is kept at a manageable level. Fast machine protection and pulsed beam operation are achieved via the chopper assembly, which consists of a kicker combined with an absorber. In some scenarios, the LEBT chopper assembly can be used also as a pre-chopper to assist the MEBT chopping system. Note that the primary machine protection mechanism is to disable the beam from the ion source by turning off its extraction and bias voltages. The LEBT chopper serves as a fast beam switch during the ion source turn-off time.

The LEBT optics design (Figure 2.3) incorporates two regions. First, the beam is nearly fully neutralized from the exit of the ion source to a point immediately upstream of the chopper kicker. Then, depending on the operational mode the beam can be either neutralized or un-neutralized from downstream of the kicker to the RFQ entrance. In the un-neutralized mode, the secondary ions created in the downstream region are removed by a constant electric field on the kicker plates, and the upstream ions are trapped by a positive voltage on an electrode inside the second solenoid, referred below as insulated diaphragm \#2. In the neutralized mode, the kicker plates as well as insulated diaphragm \#2 are normally grounded, while the scraper located at the line end is biased positively to prevent ions from escaping longitudinally. 
The LEBT scheme is flexible enough to accommodate both versions of beam neutralization by adjusting potentials and solenoid currents. Figure 2.4 presents the results of simulations for the fully neutralized transport. The transport with the un-neutralized downstream section is beneficial for minimizing the difference between short pulse and $\mathrm{CW}$ operations, as well as for minimizing transition effects when the beam is switched on. However un-neutralized beam transport may result in an emittance growth as illustrated in Figures 2.5 and Figure 2.6. The relative benefits of each scenario will be clarified in the course of PXIE experiments.

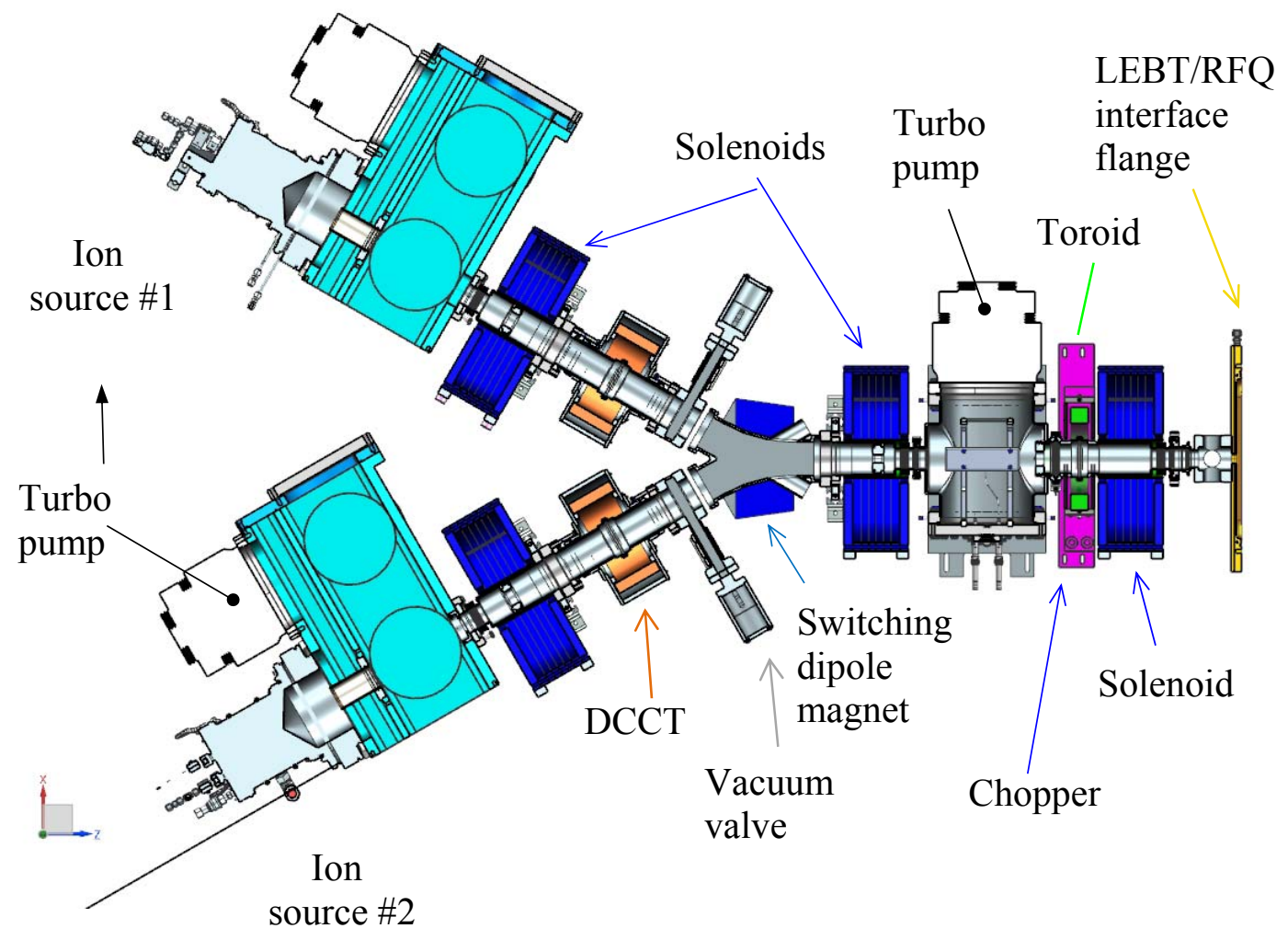

Figure 2 2. Schematic of the LEBT with two ion sources.

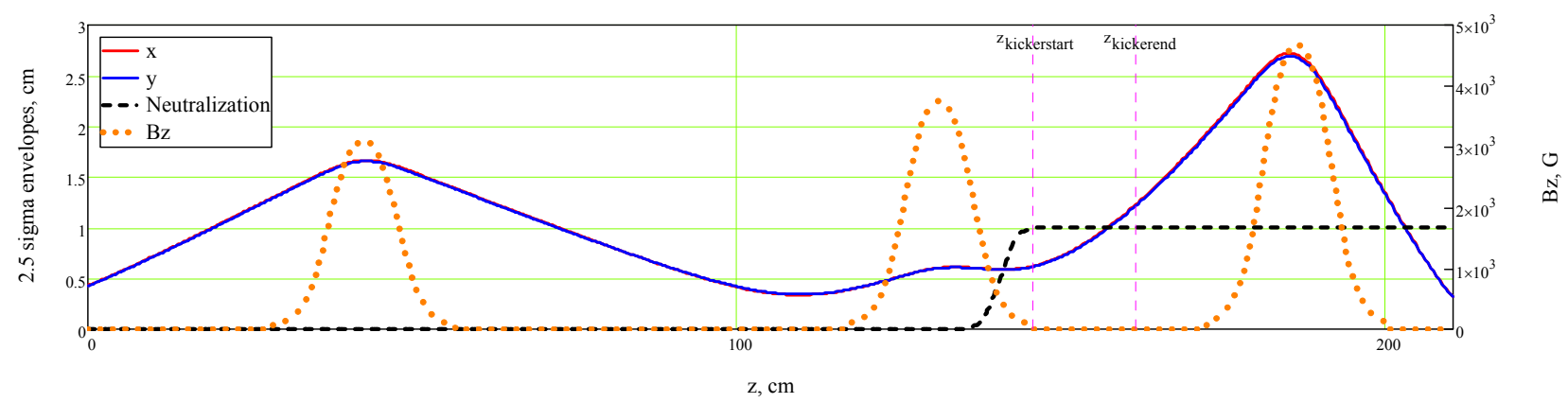

Figure 2.3: Beam horizontal (red) and vertical (blue) envelopes $(2.5 \sigma)$ for the partially unneutralized LEBT optics solution (computed with a PIC-like code written in MathCAD). The black dashed line indicates the level of neutralization $(0 \equiv$ fully neutralized; $1 \equiv$ full beam current of 10 $\mathrm{mA}$ ). The transition from 0 to 1 coincides with the position of isolated diaphragm \#2. The dotted orange line shows the longitudinal magnetic field on-axis along the beam line provided by the solenoids. 


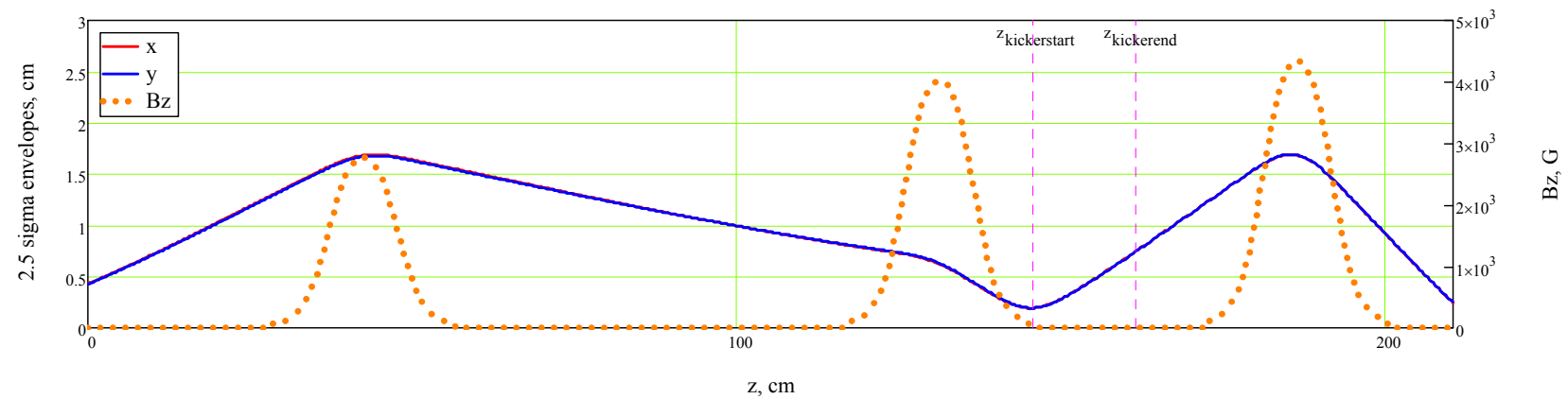

Figure 2.4: Beam horizontal (red) and vertical (blue) envelopes $(2.5 \sigma)$ in the LEBT with fully neutralized transport. All optical elements are identical to those in Figure 2.3; only the solenoid currents were adjusted.

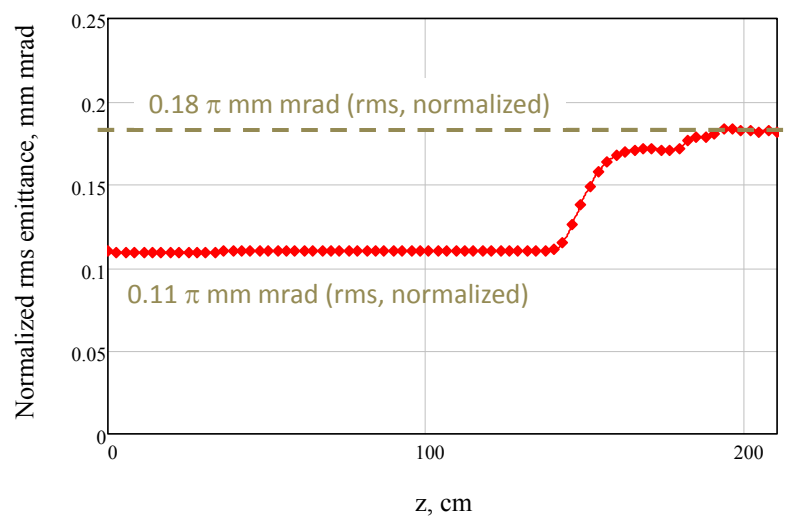

Figure 2.5: Emittance evolution along the LEBT for a $10 \mathrm{~mA}, 30 \mathrm{keV}$ beam for partially neutralized transport. The start of the emittance growth appears at the transition between the neutralized and un-neutralized beam transport. The initial distribution of the $\mathrm{H}^{-}$ions is axially symmetrical and Gaussian both in spatial and angle planes.
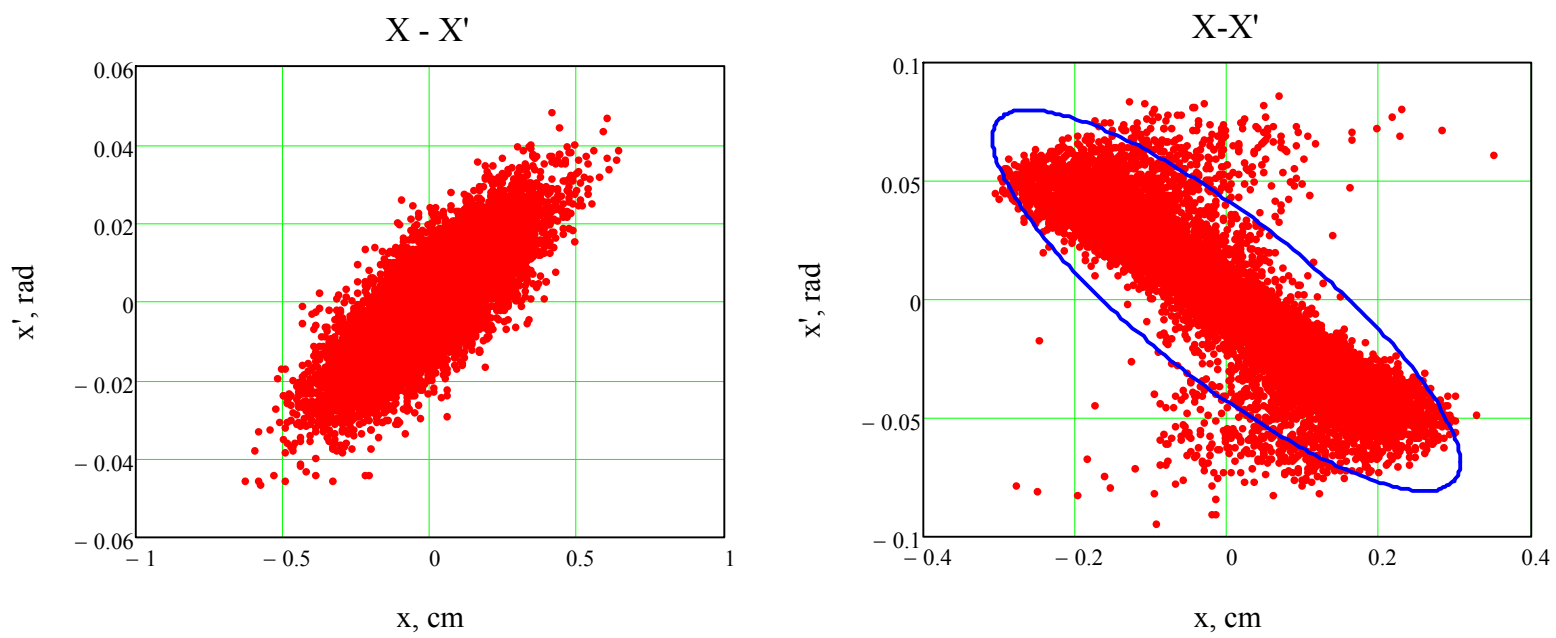

Figure 2.6: Phase space distribution $\left(x-x^{\prime}\right)$ at the entrance of the RFQ for fully (left) and partially (right) neutralized transports. The initial distribution was Gaussian in both planes. 


\subsubsection{RFQ - Radio Frequency Quadrupole Accelerator}

The $162.5 \mathrm{MHz} \mathrm{RFQ}$ accelerates the $30 \mathrm{keV} \mathrm{H}^{-}$ion beam to $2.1 \mathrm{MeV}$ for beam currents of up to $10 \mathrm{~mA} \mathrm{CW}$. Design parameters are presented in Table 2.3.

The beam dynamics of the RFQ was simulated using the beam distribution measured at the output of the D-Pace $\mathrm{H}^{-}$ion source [16], which is used to test $\mathrm{CW}$ operation of the warm frontend. Figure 2.7 presents the dependence of computed RFQ transmission on the beam current. The design has over $98 \%$ transmission for the beam current from 1 to $15 \mathrm{~mA}$. At the nominal current of $5 \mathrm{~mA}, 99.8 \%$ beam capture is achieved in this simulation.

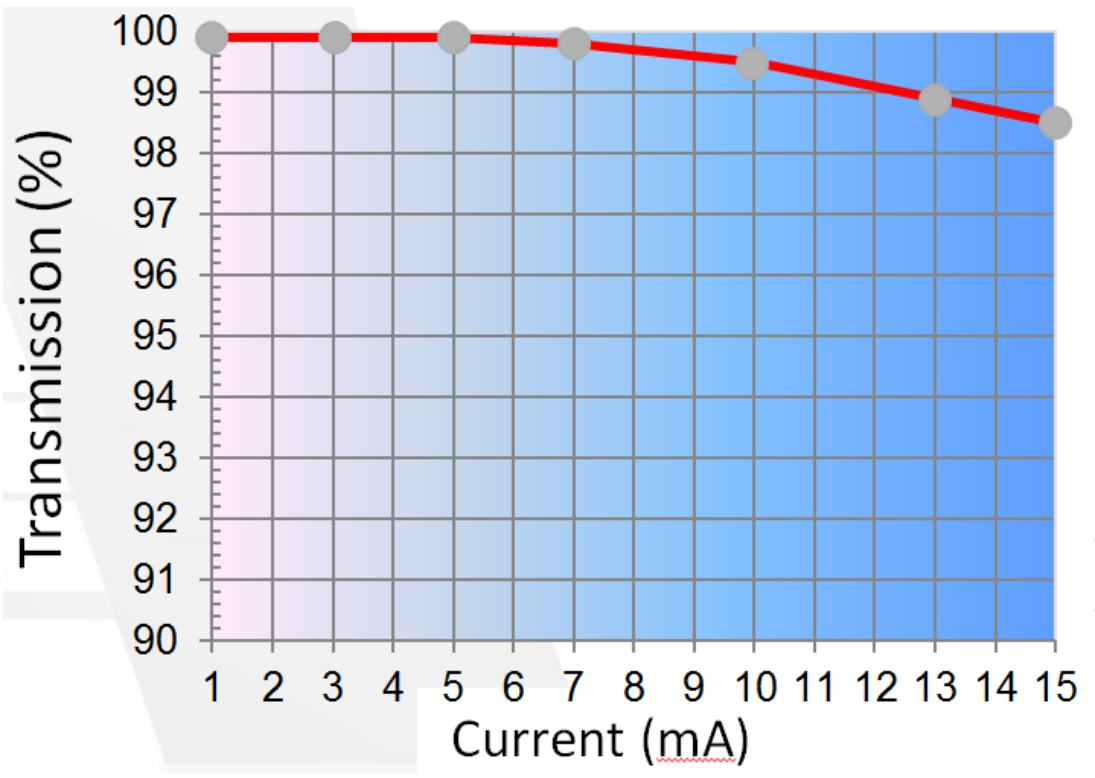

Figure 2.7: Dependence of the calculated RFQ transmission on the beam current.

Table 2.3: Main parameters of the RFQ

\begin{tabular}{lll}
\hline \hline Parameters & Value & Unit \\
\hline Input energy & 30 & $\mathrm{kV}$ \\
Output Energy & 2.1 & $\mathrm{MeV}$ \\
Duty factor & 100 & $\%$ \\
Frequency & 162.5 & $\mathrm{MHz}$ \\
Beam current & 5 (nominal); 1-10 & $\mathrm{mA}$ \\
Transmission at $5 \mathrm{~mA}$ & 99.8 & $\%$ \\
Output transverse Emittance at $5 \mathrm{~mA}$ & 0.15 & $\mathrm{~mm}-\mathrm{mrad}$ \\
Output longitudinal Emittance at $5 \mathrm{~mA}$ & 0.70 & $\mathrm{keV}$-nsec \\
\hline \hline
\end{tabular}



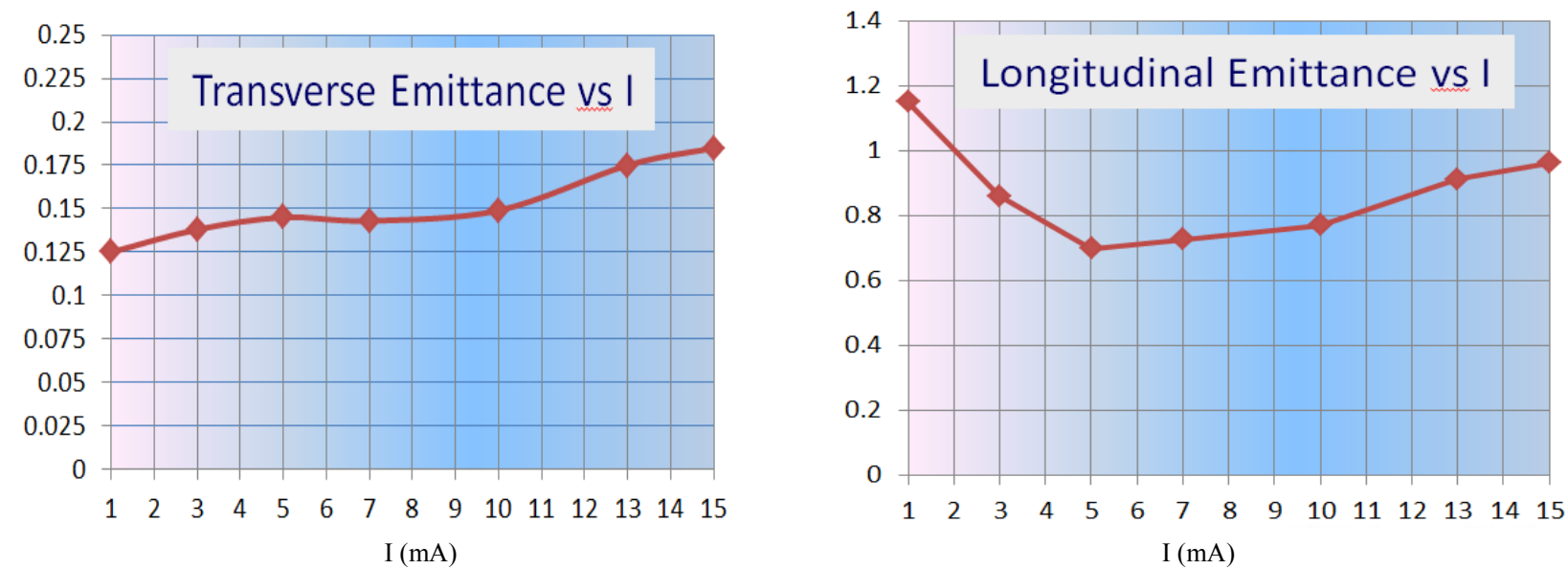

Figure 2.8: Dependence of the calculated transverse (left) and longitudinal (right) rms normalized emittances on the beam current; the transverse emittance is presented in $\mathrm{mm} \cdot \mathrm{mrad}$ and the longitudinal one in $\mathrm{keV} \cdot \mathrm{ns}(1 \mathrm{keV} \cdot \mathrm{ns} \approx 0.32 \mathrm{~mm} \mathrm{mrad})$.
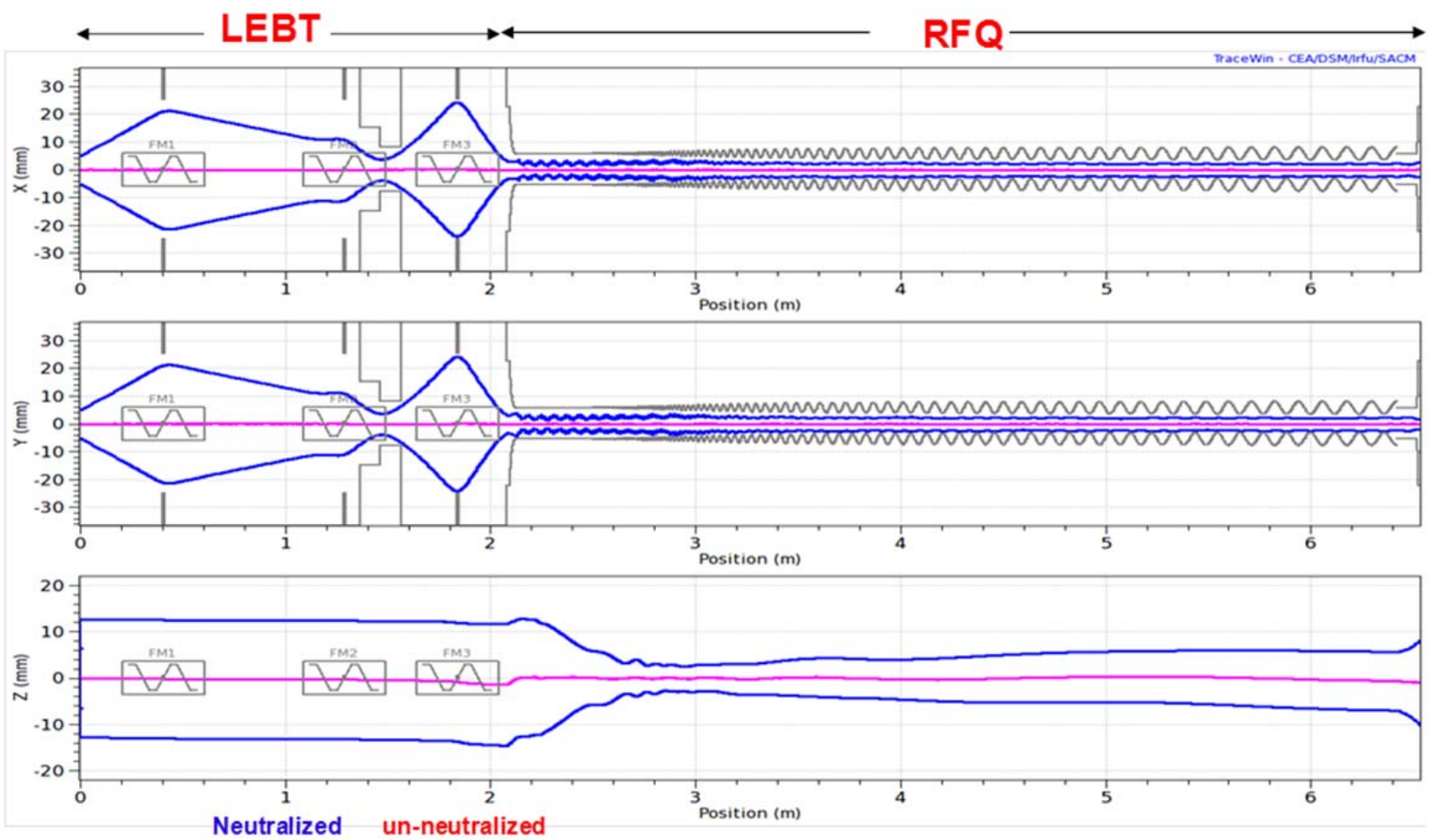

Figure 2.9: TraceWin simulations of $3 \sigma$ beam envelopes (hor, vert, and long.) through the LEBT and the RFQ for a 5-mA beam current. Magenta lines show displacements of bunch centroid.

Results of simulations of the transverse and longitudinal emittances as functions of the beam current (assuming a $0.11 \mathrm{~mm}$-mrad rms normalized emittance at the RFQ entrance) are presented in Figure 2.8. At the nominal beam current, the output rms normalized transverse and longitudinal emittances are $0.15 \mathrm{~mm}-\mathrm{mrad}$ and $0.7 \mathrm{keV}$-ns $(0.224 \mathrm{~mm}$-mrad $)$, respectively. The beam dynamics simulation was conducted using PARMTEQM and TraceWin [17, 18]. Figure 2.9 shows the simulated $3 \sigma$ beam envelopes at $5 \mathrm{~mA}$, starting at the exit of the ion source, for a fully neutralized beam current. The particles longitudinal distribution over longitudinal action is shown in Figure 2.10 . 


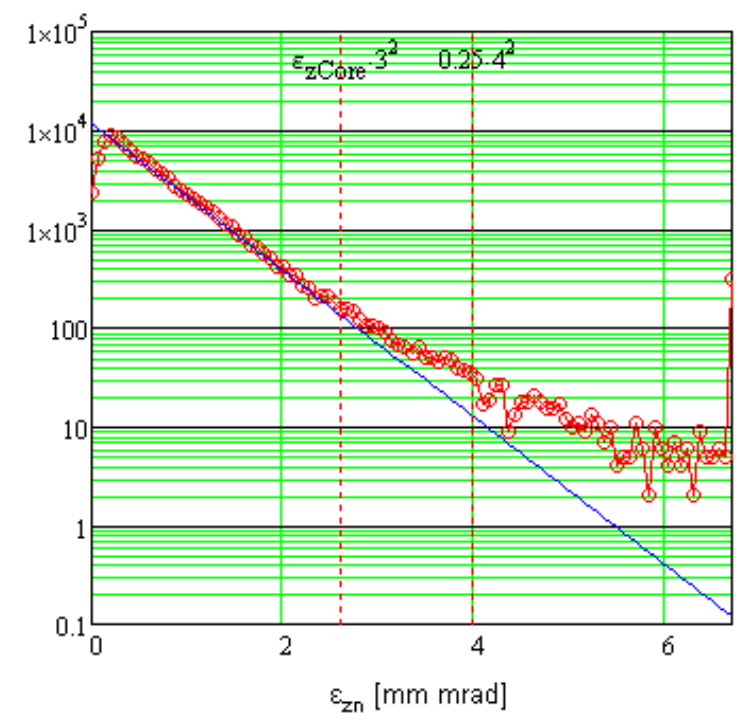

Figure 2.10: Particle longitudinal distribution at the end of the RFQ simulated for $5 \mathrm{~mA}$ beam current. Vertical lines mark the $1 \sigma$ and $4 \sigma$ boundaries for $0.25 \mathrm{~mm}$-mrad (rms) normalized longitudinal emittance.

\subsubsection{MEBT - Medium Energy Beam Transport}

The required bunch structure for PIP-II operations will be formed in the MEBT, the $\sim 10 \mathrm{~m}$ section between the RFQ and the HWR cryomodule. The heart of the MEBT is a wideband chopping system that directs unneeded bunches to an absorber according to a pre-selected pattern and transfers bunches chosen for further acceleration into the SC Linac with minimum distortions. Beam chopping in the MEBT is used in other facilities (e.g. SNS [19]), but the concept of bunchby-bunch selection results in significantly more demanding requirements to the chopping system. In addition, the MEBT provides the proper optical matching between the RFQ and the SRF section, includes tools to measure the properties of the beam coming out of the RFQ and transported to the SRF cavities, and has means of protecting the SRF section from excessive beam loss and flow of gas originating in the beam absorber.

The MEBT transports the $2.1 \mathrm{MeV}, 1-10 \mathrm{~mA} \mathrm{H}^{-}$beam with low emittance growth $(<10 \%)$ and low beam loss of the passing bunches. The complete list of functional requirements is presented in Ref. [20]. Transverse focusing in the MEBT is provided mainly by equidistantly placed quadrupole triplets with the exception of the two doublets at the RFQ exit (see Figures 2.11 and 2.12) matching the RFQ beam envelopes to the MEBT periodic focusing structure. Each triplet or doublet is followed by a pair of dipole correctors (one horizontal and one vertical). The specifications for the quadrupoles and correctors are listed in Ref. [21]. Below, the spaces between neighboring triplets or doublets are referred to as MEBT sections. The period in the regular part is $1140 \mathrm{~mm}$, which leaves a $650-\mathrm{mm}$ long (flange-to-flange) space for various equipment (only $350 \mathrm{~mm}$ in the section between doublets labeled \#0 in Figure 2.11).

The undesired beam bunches will be removed in the MEBT by a chopping system, shown in Figure 2.11 by the pink boxes. The chopper consists of two identical $50 \mathrm{~cm}$ long kickers separated by a $180^{\circ}$ betatron phase advance and an absorber $\left(90^{\circ}\right.$ from the last kicker). In the broadband, travelling-wave kicker, the transverse electric field propagates with the phase velocity equal to the speed of $\mathrm{H}^{-}$ions $(\sim 20 \mathrm{~mm} / \mathrm{ns}, \beta=0.0668)$ so that the ion vertical velocities change sufficiently to displace the ion bunches, that are designated for removal, onto the absorber. Detailed specifications 
for the kicker can be found in Ref. [22]. Figure 2.12 presents the simulated transverse beam envelopes in the MEBT for both passing and chopped bunches. The chopped bunches are directed onto an absorber which is displaced vertically from the beam trajectory. Presently two versions of the kicker, which differ by the structure's impedance, are being investigated [23]. To keep the beam properly bunched, the MEBT includes 3 identical bunching cavities [24].

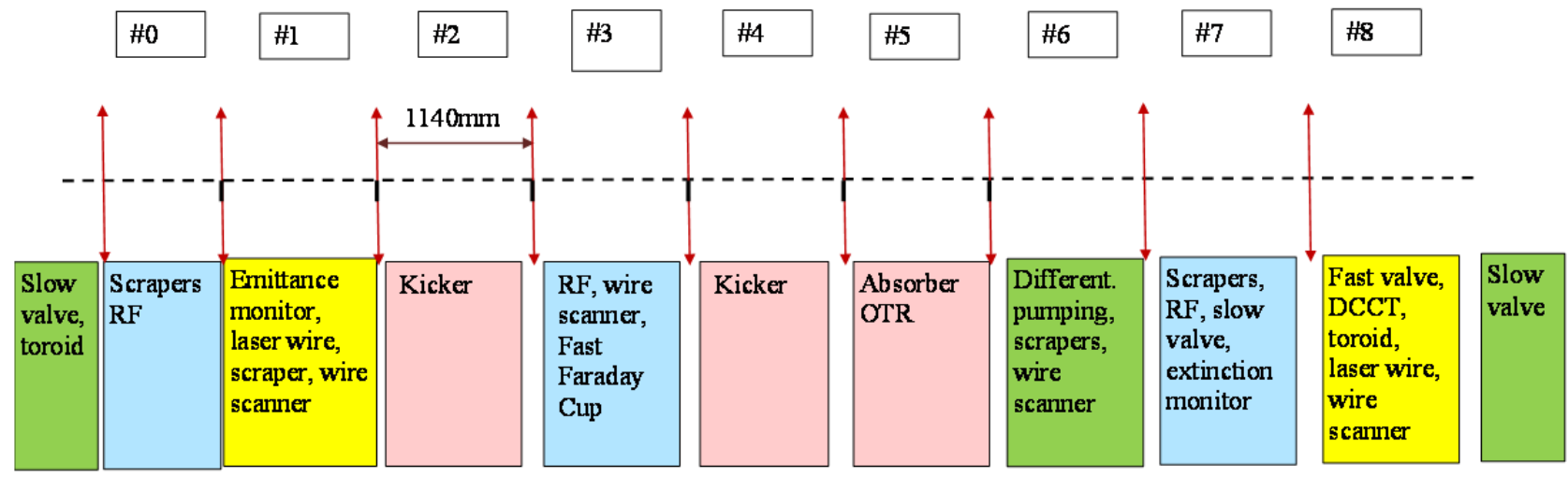

Figure 2.11: The MEBT structure. Sections are color-coded according to their main functions. The red vertical arrows schematically show the transverse focusing (doublets or triplets) elements.
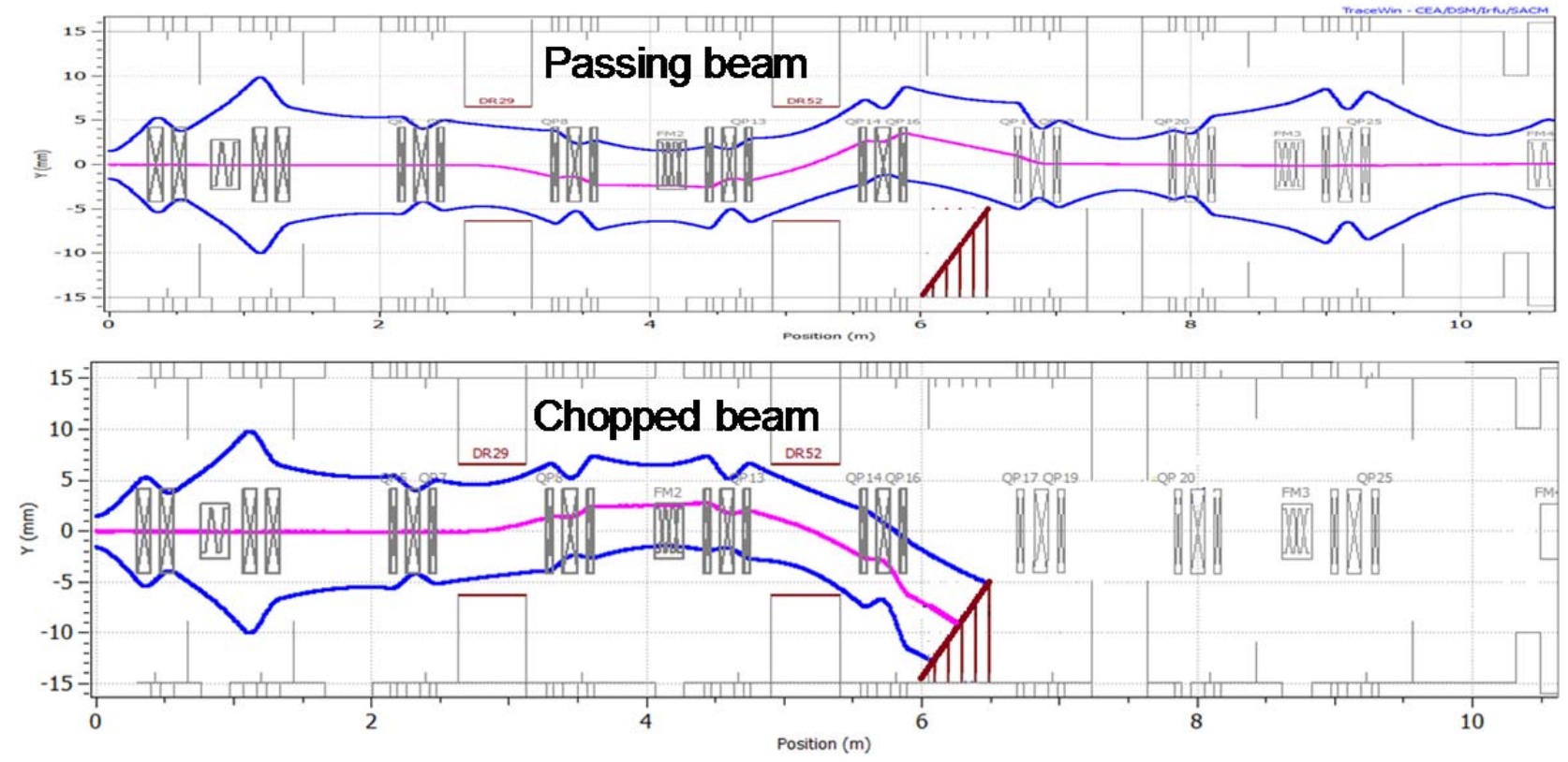

Figure 2.12: Simulated 3 $\sigma$ transverse beam envelopes in the MEBT. 


\subsubsection{SC Linac - Superconducting Linac}

The SC Linac starts immediately downstream of the MEBT. It accelerates the beam from 2.1 $\mathrm{MeV}$ to $0.8 \mathrm{GeV}$ and includes five types of superconducting (SC) cavities to cover the entire velocity range required for acceleration of $\mathrm{H}^{-}$(or protons).

\subsubsection{Accelerating Cavities}

The cavity frequencies and cell configuration are selected to maximize acceleration efficiency for each accelerating structure, to minimize cost of the accelerator and its operation, and to minimize the beam loss.

A primary efficiency factor for a cavity is the flight factor, $T(\beta)$, which has a dependence on the beam velocity, $\beta$, shown in Figure 2.13 for different numbers of cells in a cavity. The figure shows that the range in $\beta$ over which the beam can be efficiently accelerated increases with a decreasing number of cells per cavity. On the other hand, a too small number of cells reduces the effective gradient and increases costs, due to end effects. The maximum acceleration is achieved for a velocity larger than the geometric-beta, $\beta_{G}$. This velocity for maximal acceleration is called the optimal beta, $\beta_{\text {opt }}$. For a periodic structure with a harmonic distribution of electric field along the axis,

$$
E \propto \sin \left(\omega z / \beta_{G} c\right) \exp (i \omega t),
$$

the flight factor can be expressed by the following formula:

$$
T(\beta)=\frac{T_{0}(\beta)}{T_{0}\left(\beta_{\text {opt }}\right)}, \quad T_{0}(\beta)=\frac{2 \beta}{\pi n}\left(\frac{\sin \left(\pi n\left(\beta-\beta_{G}\right) /(2 \beta)\right)}{\beta-\beta_{G}}-(-1)^{n} \frac{\sin \left(\pi n\left(\beta+\beta_{G}\right) /(2 \beta)\right)}{\beta+\beta_{G}}\right),
$$

where $n$ is the number of cells in the cavity operating at $\pi$-mode. $T_{0}(\beta)$ is the transit-time factor, which is normalized so that $T_{0}\left(\beta_{G}\right)=1$, while the flight factor is normalized at $T\left(\beta_{\text {opt }}\right)=1$. The above expression approximates well the transit-time factors obtained by numerical integration of the actual time dependent electric field of the PIP-II cavities. The geometric betas, $\beta_{G}$, presented in Table 2.4 were obtained by fitting Eq. (2.2) to the numerical integration results. Note that for typical multicell elliptic resonators an accurate accounting of fields in the edge cavities results in a value of $\beta_{G}$ slightly larger than the other frequently used definition based on Eq. (2.1), where $\beta_{G}$ is defined as the ratio of cavity period to the half-wavelength. The corresponding numbers are presented in the note to Table 2.4. For a large number of cells per cavity the geometric and optimal betas of Eq. (2.2) are related by the following approximate equation:

$$
\beta_{\text {opt }} \approx \beta_{G}\left(1+\frac{6}{\pi^{2} n^{2}}\right) \text {. }
$$

Recent developments in $1300 \mathrm{MHz}$ ILC technology at Fermilab [25] and elsewhere have significantly improved SRF technology in general and have made it a preferable choice for the possible future extension of the PIP-II linac to higher energy. That forces the choice of accelerating frequencies to be subharmonics of the ILC frequency of $1300 \mathrm{MHz}$, and, consequently, yields 162.5, 325 and $650 \mathrm{MHz}$ as frequencies for PIP-II. This choice results in a comparatively smooth frequency increase in the course of acceleration, accommodating bunch compression due to adiabatic damping.

Table 2.4 and Figure 2.14 present parameters of the cavities for the linac. The acceleration starts with half-wave resonators (HWR) operating at $162.5 \mathrm{MHz}$. These are followed by two types of single spoke resonators operating at $325 \mathrm{MHz}$ (SSR1 and SSR2), and finally by two types of 
elliptical 5-cell cavities at $650 \mathrm{MHz}$ (LB650 and HB650). Figure 2.15 presents the flight-time factors for the SC Linac. The accelerating voltage in each next cavity type is significantly larger than in the previous one. That determines that the transition happens earlier than the transit-time factors for two types become equal.

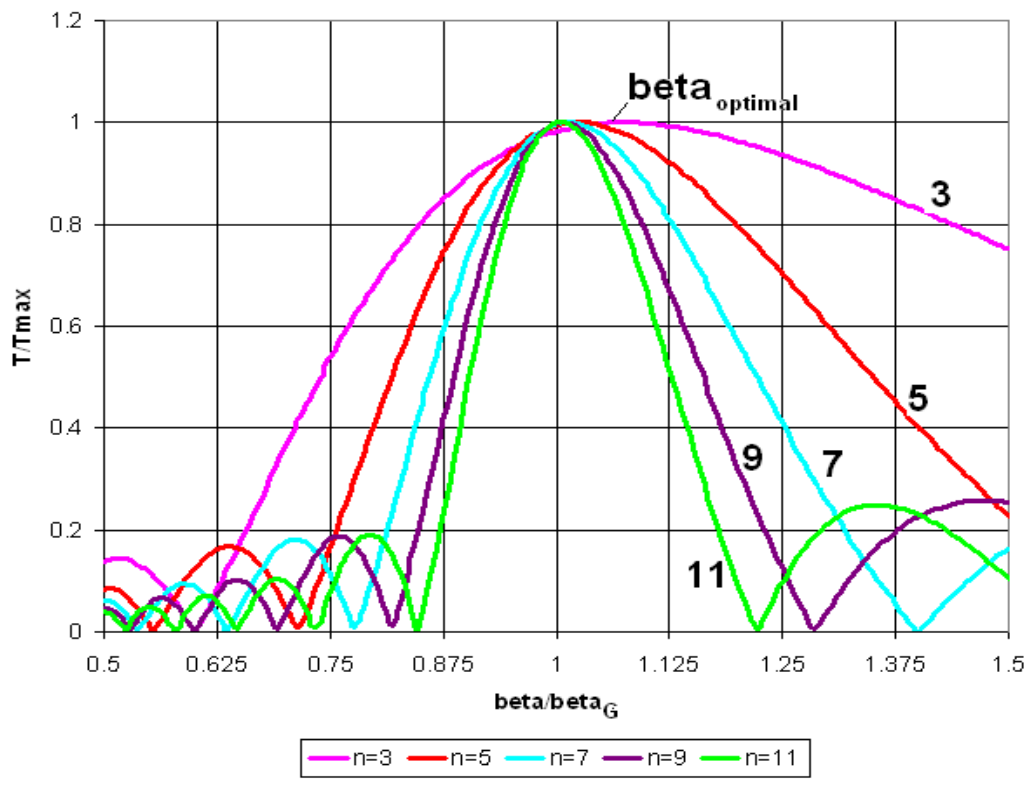

Figure 2.13: Transit-time factor versus the ratio of the beta to the geometrical beta, $\beta / \beta_{G}$, for different number of cells in a cavity, $n$.

Table 2.4: Accelerating cavities types for the SC Linac

\begin{tabular}{l|c|c|c|c|c|c}
\hline $\begin{array}{l}\text { Cavity } \\
\text { type }\end{array}$ & $\beta_{\mathrm{G}}$ & $\beta_{\mathrm{opt}}$ & $\begin{array}{c}\text { Freq. } \\
(\mathrm{MHz})\end{array}$ & $\begin{array}{c}\text { Type } \\
\text { of cavities }\end{array}$ & $\begin{array}{c}\text { Energy gain at } \beta_{\text {opt }} \\
\text { per cavity }(\mathrm{MeV})\end{array}$ & $\begin{array}{c}\text { Energy range } \\
(\mathrm{MeV})\end{array}$ \\
\hline HWR & 0.094 & 0.112 & 162.5 & Half wave resonator & 2 & $2.1-10.3$ \\
\hline SSR1 & 0.186 & 0.222 & 325 & $\begin{array}{c}\text { Single-spoke } \\
\text { resonator }\end{array}$ & 2.05 & $10.3-35$ \\
\hline SSR2 & 0.398 & 0.475 & 325 & $\begin{array}{c}\text { Single-spoke } \\
\text { resonator }\end{array}$ & $55-185$ \\
\hline LB650 & $0.631^{*}$ & 0.647 & 650 & Elliptic 5-cell cavity & $11.9\left(11.7^{\star}\right)$ & $185-500$ \\
\hline HB650 & $0.947^{*}$ & 0.971 & 650 & Elliptic 5-cell cavity & $19.9\left(19.6^{\star}\right)$ & $500-800$ \\
\hline \hline
\end{tabular}

* Note that $\beta_{G}$ for the elliptic cavities can be also defined as the ratio of regular cell length to halfwavelength. That yields $\beta_{G}=0.61$ for LB650 and yields $\beta_{G}=0.92$ for HB650.

- It represents a mean value among different field distributions with field flatness of $95 \%$.

The choice of the RFQ frequency was determined by a requirement for the possibility of bunchby-bunch chopping which would be beyond the present "state-of-the art" at $325 \mathrm{MHz}$ but is feasible for $162.5 \mathrm{MHz}$. The same frequency is used for the first superconducting cryomodule (HWR), because it results in reduced transverse defocusing and reduced longitudinal focusing from 
cavity fields, which otherwise would severely limit the accelerating gradient in the first SC cryomodule. The number of cavities, and the linac length, required to accelerate the beam to 11 $\mathrm{MeV}$ is reduced by more than a factor of 2, compared to cryomodules with $325 \mathrm{MHz}$ cavities. Note that even this frequency choice does not enable a usage of nominal voltage for the first few cavities. In particular, the first cavity uses about half of nominal voltage.

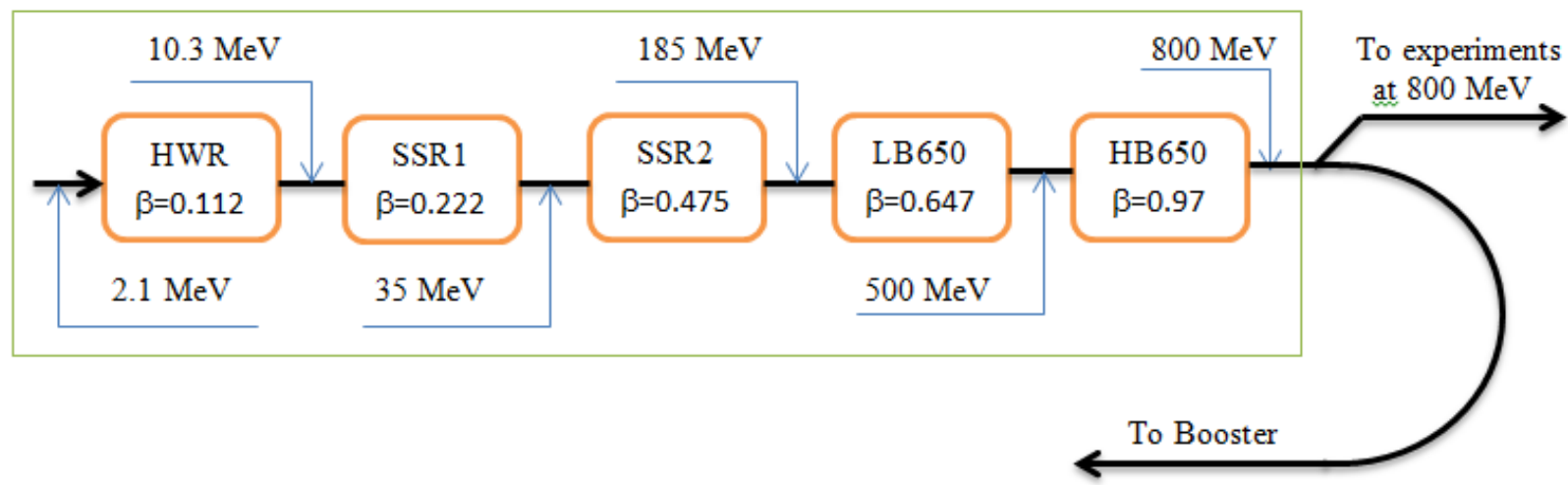

Figure 2.14: Technology map for SC part of PIP-II linac.

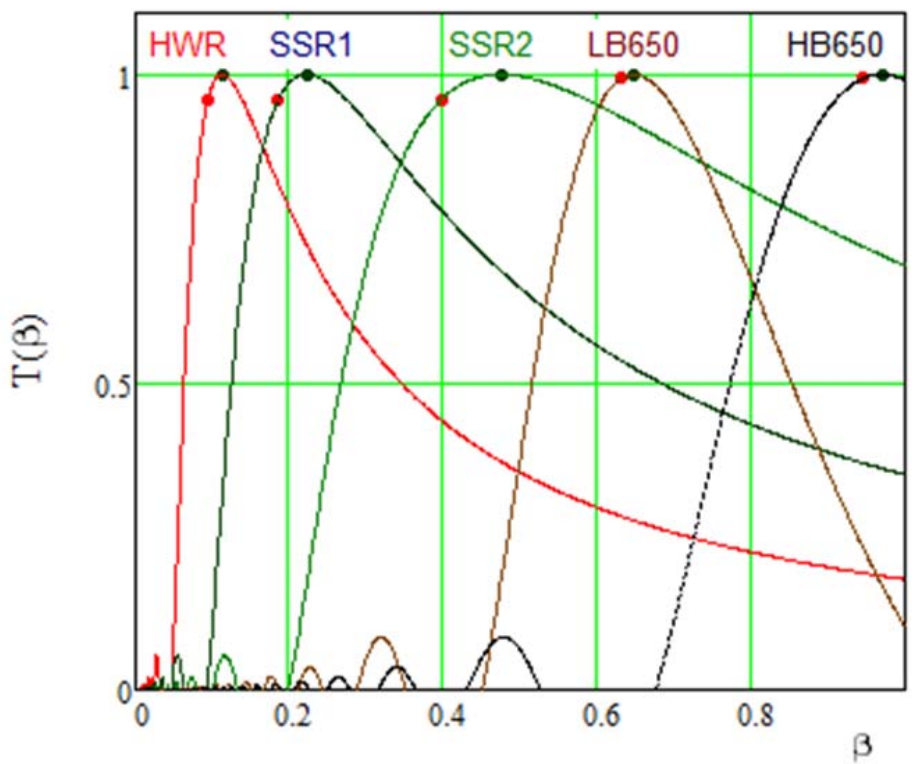

Figure 2.15: Transit-time factors for PIP-II SC cavities; red dots mark position of $\beta_{G}$, and blue dots position of $\beta_{\text {opt }}$.

The cavity arrangement described above also yields:

- simplified longitudinal beam dynamics at each transition from one cavity type to another due to the limitation of frequency jumps to a factor of two, and

- an increased aperture at the beginning of the linac due to the use of lower frequency sections.

The larger apertures reduce uncontrolled beam loss on low temperature surfaces to a tolerable level. We emphasize that the choice of cavities with lower frequencies reduces the effects of focusing/defocusing by accelerating cavities, and also decreases the number of cells per cavity, 
consequently widening the dependences of transit-time factors on beta, which increases effective accelerating gradients and acceleration efficiency.

However, there are also some disadvantages related to the preference for lower frequencies:

- Microphonics is a more serious issue at lower frequencies.

- Lower frequency cavities are more expensive (more niobium), but that cost increase is compensated (within presently known accuracy) by the use of a smaller number of cavities and RF sources. The latter is mainly related to a smaller number of cells for the elliptic cavities ( 5 versus 9 for the ILC cavities), and, consequently, smaller variation of the transittime factors.

The operating gradient is chosen to provide a peak surface magnetic field that allows operation below high-field Q-slope; see Figure 2.16 taken from Ref. [26] (see also [27]). For the frequency of 162.5 MHz we adopt a the maximum magnetic field of about $50 \mathrm{mT}$; while for the frequencies of $650 \mathrm{MHz}$ it increases to about $70 \mathrm{mT}$. For all frequencies the peak surface electric field is less than $40 \mathrm{MV} / \mathrm{m}$ [28] in order to avoid the risk of strong field emission (see details in Section 3). The transition from the $325 \mathrm{MHz}$ single-spoke cavities to the $650 \mathrm{MHz}$ section based on elliptical cavities is chosen at the energy of about $185 \mathrm{MeV}$, because at lower energies elliptical cavities lose efficiency. It is inefficient to accelerate $\mathrm{H}^{-}$ions from $170 \mathrm{MeV}$ to $0.8 \mathrm{GeV}$ using only one cavity type and, thus, two families of $650 \mathrm{MHz}$ cavities are chosen. Table 2.5 presents the main electrodynamical parameters of SC cavities. The effective length of a cavity is computed based on $\beta_{\text {opt }}$ so that: $L_{\text {eff }}=n_{\text {cell }} \beta_{\text {opt }} c /(2 f)$, where $n_{\text {cell }}$ is the number of cells in a cavity $\left(n_{\text {cell }}=2\right.$ for HWR, SSR1 and SSR2; $n_{\text {cell }}=5$ for LB650 and HB650). Consequently, the accelerating gradient is $\Delta E / L_{\text {eff }}$, where $\Delta E$ is the net energy gain at the optimal beta.

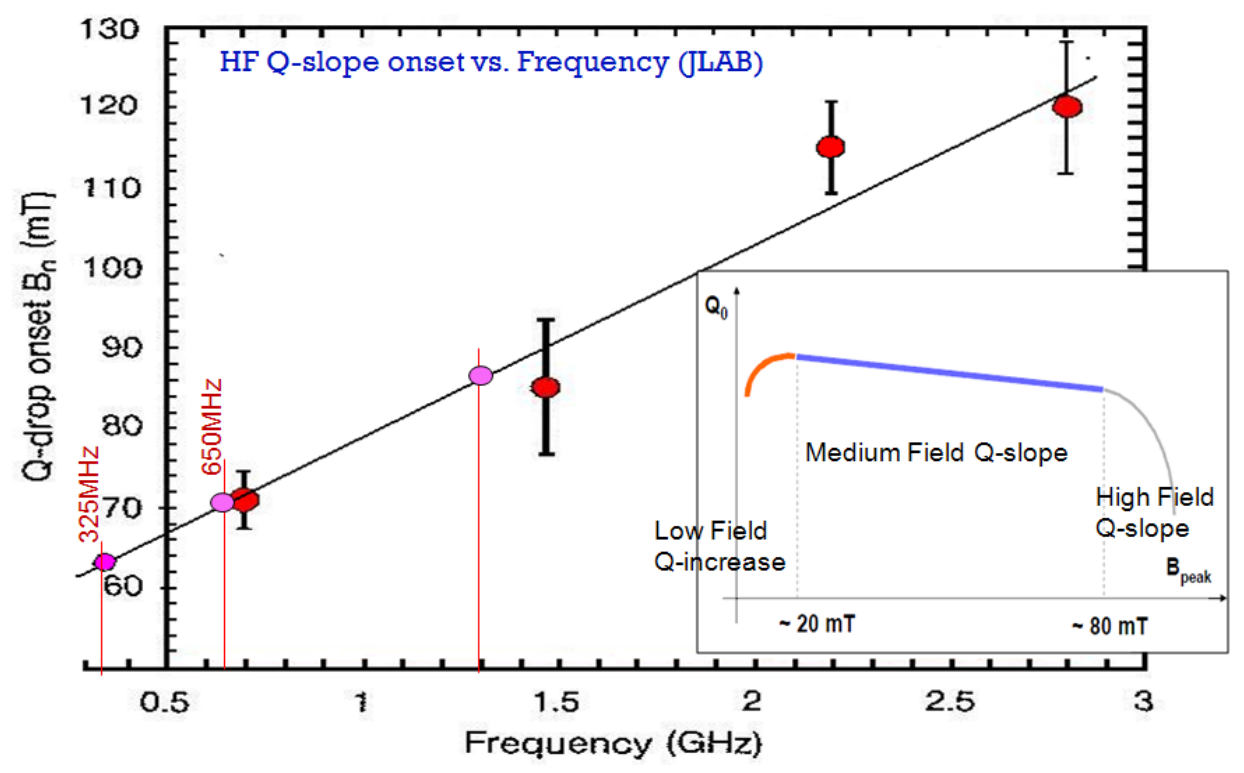

Figure 2.16: High field Q-slope versus frequency.

The transition energies between different types of cavities were optimized to minimize the total number of cavities. As an example of such optimization Figure 2.17 displays the number of cavities required as a function of geometrical betas for LB650 and HB650. Here we additionally assume a linear dependence of the field enhancement factors versus beta [29], that the initial synchronous phase is $-30^{\circ}$, and its modulus decreases inversely proportional to the square root of the energy to 
keep the desired RF bucket size. As one can see from the left pane of Figure 2.17 the number of cavities has a weak dependence on betas in vicinity of the minimum. The optimal geometrical betas for the two $650 \mathrm{MHz}$ sections are 0.64 and 0.9 respectively (left), and the optimal transition energy is $466 \mathrm{MeV}$ (right). More accurate simulations taking into account realistic enhancement factors obtain an optimal choice of betas at 0.61 and 0.9 .

Table 2.5: Main electro-dynamical parameters of SC cavities

\begin{tabular}{l|c|c|c|c|c|c|c}
\hline \hline $\begin{array}{l}\text { Cavity } \\
\text { type }\end{array}$ & $\begin{array}{c}\text { Aperture } \\
(\text { diameter }) \\
(\mathrm{mm})\end{array}$ & $\begin{array}{c}\text { Effective } \\
\text { length } \\
(\mathrm{cm})\end{array}$ & $\begin{array}{c}\text { Accelerating } \\
\text { gradient } \\
(\mathrm{MV} / \mathrm{m})\end{array}$ & $\begin{array}{c}\mathrm{E}_{\text {peak }}{ }^{*} \\
(\mathrm{MV} / \mathrm{m})\end{array}$ & $\begin{array}{c}\mathrm{B}_{\text {peak }}{ }^{*} \\
(\mathrm{mT})\end{array}$ & $\begin{array}{c}(\mathrm{R} / \mathrm{Q})^{3} \\
(\Omega)\end{array}$ & $\begin{array}{c}\mathrm{G} \\
(\Omega)\end{array}$ \\
\hline HWR & 33 & 20.7 & 9.7 & 44.9 & 48.3 & 275 & 48 \\
\hline SSR1 & 30 & 20.5 & 10 & 38.4 & 58.1 & 242 & 84 \\
\hline SSR2 & 40 & 43.8 & 11.4 & 40 & 64.5 & 296 & 115 \\
\hline LB650 & 83 & 74.6 & 15.9 & 38.5 & 72 & 375 & 191 \\
\hline HB650 & 118 & 111.9 & 17.8 & 38.3 & 72 & 609 & 260 \\
\hline \hline
\end{tabular}

${ }^{*}$ For energy gain per cavity presented in Table 2.4 .
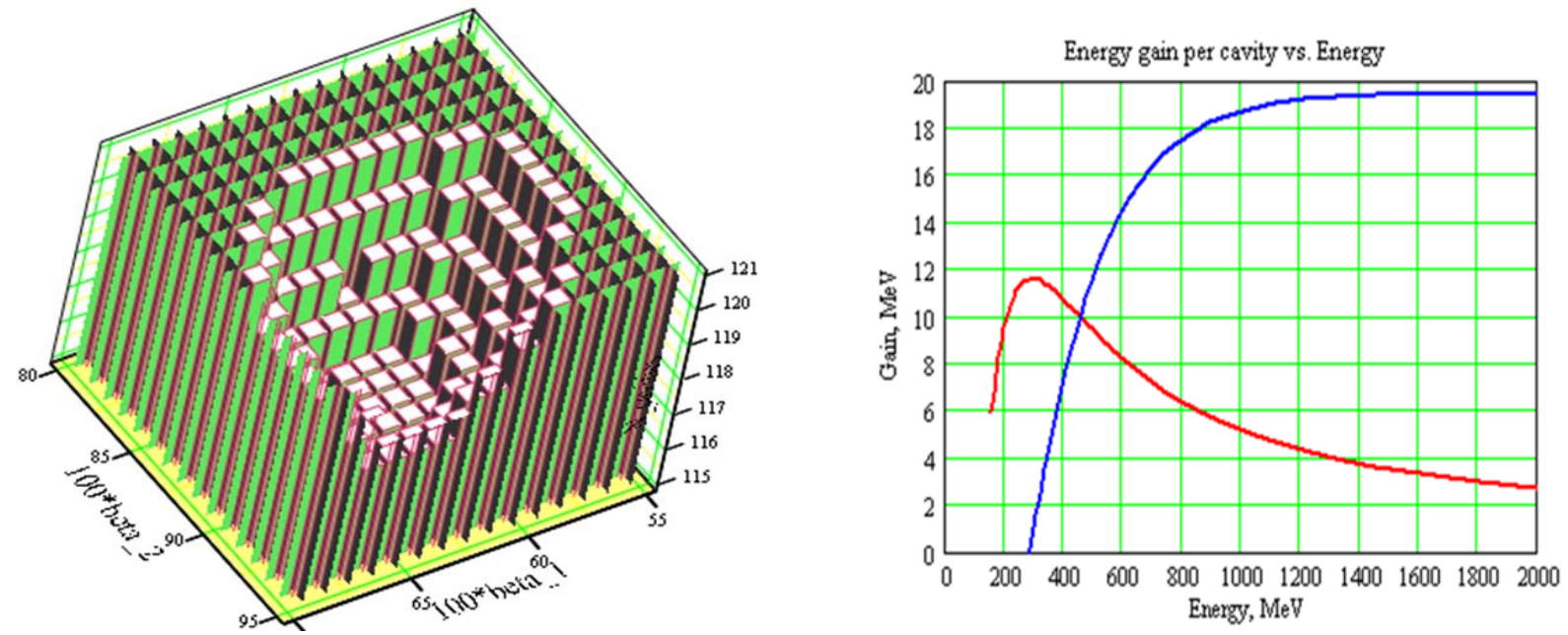

Figure 2.17: Number of cavities required versus cavity beta in the two $650 \mathrm{MHz}$ sections (left) and the energy gain per cavity versus particle energy (right) for LB650 (red curve) and HB650 (blue curve) cavities.

\subsubsection{SC Cryomodules and Requirements to their Cryogenics}

Cavities and focusing elements, as necessary, are grouped within cryomodules. In the 162.5 and $325 \mathrm{MHz}$ sections transverse focusing is provided by superconducting solenoids, while in the 650 $\mathrm{MHz}$ sections by normal conducting quadrupole doublets located outside of the cryomodules. The

${ }^{3}$ Through this document we define $(R / Q)$ so that in the absence of cavity detuning and beam current, the RF power required to create the voltage amplitude $U_{0}$ is equal to: $P_{g}=\left(1+\beta_{c}{ }^{2}\right)^{2} U_{0}{ }^{2} /\left(4 \beta_{c}(R / Q) Q_{0}\right)$, where $\beta_{c}$ is the coupling coefficient, and $Q_{0}$ is the cavity unloaded quality factor. 
main cryomodule parameters and the arrangement of focusing periods by cryomodule type are shown in Table 2.6. The periodicity of focusing elements is chosen to achieve sufficiently strong focusing required to reduce focusing variations due to variation of cavity transverse defocusing with longitudinal particle position inside bunch. The distance between cavities in the HWR, SSR1 and SSR2 cryomodules is minimized to avoid longitudinal overfocusing representing severe limitation on the accelerating gradients at the beginning of each cryomodule type.

For beam steering and optics measurements each magnet package (i.e. solenoid or quadrupole doublet) includes vertical and horizontal correctors and a 3-coordinates beam position monitor ${ }^{4}$ (BPM). All cryomodules are separated by warm sections. These warm sections are used for additional diagnostics (bunch transverse and longitudinal profile monitors, beam loss monitors, etc.) and for beam collimators required to avoid uncontrolled beam loss inside SC cryomodules. The makeup for each of the warm insertions will be determined by requirements of safe and reliable operations, diagnostics, collimation, and cryogenic segmentation constraints. Details of cryomodule designs are presented in Section 3.

Table 2.6: General parameters of SC cryomodules

\begin{tabular}{l|c|c|c|c|c|c|c}
\hline \hline $\begin{array}{l}\text { CM } \\
\text { type }\end{array}$ & $\begin{array}{c}\text { Cavities } \\
\text { per CM }\end{array}$ & $\begin{array}{c}\text { Number } \\
\text { of CMs }\end{array}$ & $\begin{array}{c}\text { CM configu- } \\
\text { ration }^{*}\end{array}$ & $\begin{array}{c}\text { CM length } \\
(\mathrm{m})\end{array}$ & $\begin{array}{c}Q_{0} \text { at } 2 \mathrm{~K} \\
\left(10^{10}\right)\end{array}$ & $\begin{array}{c}\text { Surface resis- } \\
\text { tance, }(\mathrm{n} \Omega)\end{array}$ & $\begin{array}{c}\text { Loaded } Q \\
\left(10^{6}\right)\end{array}$ \\
\hline HWR & 8 & 1 & $8 \times(\mathrm{sc})$ & 5.93 & 0.5 & $9.6\left(2.75^{\star}\right)$ & 2.7 \\
\hline SSR1 & 8 & 2 & $4 \times(\mathrm{csc})$ & 5.2 & 0.6 & $14\left(10^{\#}\right)$ & 3.7 \\
\hline SSR2 & 5 & 7 & sccscsc & $6.5^{\star}$ & 0.8 & 14.4 & 5.8 \\
\hline LB650 & 3 & 11 & $\operatorname{ccc}$ & $3.9^{\star}$ & 1.5 & 12.7 & 11.3 \\
\hline HB650 & 6 & 4 & ccccc & $9.5^{\star}$ & 2 & 13 & 11.5 \\
\hline
\end{tabular}

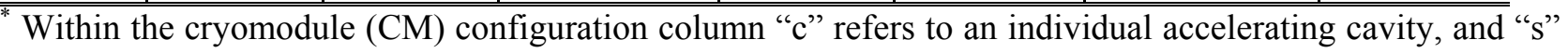
to a focusing solenoid.

- This number represents the present estimate of cryomodule length. It will be finalized with advances in the cryomodule design.

• Based on recent measurements of two HWR cavities at $2 \mathrm{MV}$ accelerating voltage.

${ }^{\#}$ Based on recent measurements of SSR1 cavities made of CABOT niobium. We expect to get better results for the SSR2 cavities to be made of material which satisfies Fermilab specifications [30].

The cavity $\mathrm{Q}_{0}$ 's are based on an operating temperature of $2 \mathrm{~K}$ and a conservative approach to the surface resistance based on values already obtained in operating cryomodules. As shown in Table 2.7 the dynamic cryo-loads in the $\mathrm{CW}$ regime significantly exceed the static loads.

The duty factor of cryogenic operation in the pulsed regime is mainly determined by the time required to pump the energy into the cavity and then to discharge it. For this estimate we assume that $Q_{0}$ does not depend on the field value. The duty factor for cryogenic operation is then equal to:

\footnotetext{
${ }^{4}$ The BPM has 4 plates and allows measurements of both transverse beam positions, as well as longitudinal bunch position measured by bunch arrival time.
} 


$$
\eta_{\text {cryo }}=\frac{f_{\text {rep }}}{E_{\max }^{2}} \int_{\substack{\text { over } \\ \text { pulse }}}(E(t))^{2} d t
$$

where $E_{\max }$ is the accelerating voltage of a cavity, and $f_{\text {rep }}$ is the repetition rate. Further we assume that the cavity voltage changes as: $E(t)=2 E_{\max }\left(1-e^{-t / 2 \tau}\right), 0<t<2 \tau \ln 2$ at cavity charging, and as $E(t)=E_{\max } e^{-t / 2 \tau}, t>0$ at cavity discharging, where $\tau=Q_{L} / 2 \pi f$ is the time constant for energy decay. The accelerating voltage stays constant during the beam pulse of $0.55 \mathrm{~ms}$.

Table 2.8 presents the cryo-duty factors and the dynamic cryo-loads assuming all cavities operate at the nominal voltages presented in Table 2.4. The static losses are the same as for CW operation and are presented in Table 2.7. As one can see for the normal cavity discharge described above the cryo-duty factor is almost an order of magnitude larger than the beam duty factor of $1.1 \%$. The cavity discharge can be accelerated by pumping the RF power with an inverted phase to the cavity after the beam pulse until the cavity voltage is zero, $E(t)=E_{\max }\left(e^{-t / 2 \tau}-2\left(1-e^{-t / 2 \tau}\right)\right), 0<t / \tau<0.8109 \ldots$. This reduces the dynamic cryo-loads by almost factor of 2 . The cavity couplers are designed to withstand the resulting four-time increase in RF power. The HWR cavities are designed so that they cannot be used in a pulsed regime and therefore are excluded from Table 2.8.

Table 2.7: Cryogenic loads in SC cryomodules for operation in the $\mathrm{CW}$ regime

\begin{tabular}{l|c|c|c|c|c|c}
\hline \hline \multirow{2}{*}{$\begin{array}{l}\text { CM } \\
\text { type }\end{array}$} & \multirow{2}{*}{$\begin{array}{l}\text { Number } \\
\text { of CMs }\end{array}$} & \multicolumn{3}{|c|}{ Static loads per CM, (W) } & $\begin{array}{c}\text { Dynamic loads per } \\
\text { CM, (W) }\end{array}$ & $\begin{array}{c}\text { Total load at 2 K } \\
\text { per CM, (W) }\end{array}$ \\
\cline { 3 - 7 } & $70 \mathrm{~K}^{*}$ & $5 \mathrm{~K}^{*}$ & $2 \mathrm{~K}$ & $2 \mathrm{~K}$ & $2 \mathrm{~K}$ \\
\hline HWR & 1 & 250 & 60 & 14 & 23.3 & 37 \\
\hline SSR1 & 2 & 194 & 71 & 12 & 23.1 & 35 \\
\hline SSR2 & 7 & 145 & 50 & 9 & 52.5 & 62 \\
\hline LB650 & 11 & 64 & 8.7 & 3 & 75.5 & 79 \\
\hline HB650 & 4 & 118 & 17.2 & 5 & 195 & 200 \\
\hline Total & & $\mathbf{2 8 2 8}$ & $\mathbf{7 1 5}$ & $\mathbf{1 4 6}$ & $\mathbf{2 0 4 8}$ & $\mathbf{2 1 9 4}$ \\
\hline \hline
\end{tabular}

- Cryo-loads are computed with nominal accelerating voltage for all cavities. The actual voltages required by the optics are smaller (see Figure 2.22) which yields somewhat smaller heating loads if all the cavities are operating at the design accelerating gradients.

* Static cryo-load includes heat flux from the couplers and current leads of magnets operating at their maximum currents.

To minimize cost of the cryogenic system the PIP-II linac will operate in pulsed mode, with the capability to be upgraded to $\mathrm{CW}$ operations at a later time. That allows utilizing some equipment of existing Tevatron cryogenic infrastructure. The projected cooling power of such a cryo-plant is presented in Table 2.9. Details of the technical implementation are presented in Section 3. A future upgrade to $\mathrm{CW}$ operation will require a new $2 \mathrm{~K}$ cryogenic plant. 
Table 2.8: Cavity parameters for operation in the pulsed regime

\begin{tabular}{|c|c|c|c|c|c|}
\hline \multirow{2}{*}{$\begin{array}{l}\mathrm{CM} \\
\text { type }\end{array}$} & \multirow{2}{*}{$\begin{array}{c}\text { Time } \\
\text { constant } \\
\tau,(\mathrm{ms})\end{array}$} & \multicolumn{2}{|c|}{ Normal cavity discharge } & \multicolumn{2}{|c|}{ Accelerated cavity discharge } \\
\hline & & $\begin{array}{l}\text { Cryo-duty } \\
\text { factor, } \%\end{array}$ & $\begin{array}{c}\text { Dynamic cryo-loads } \\
\text { per CM (W) }\end{array}$ & $\begin{array}{l}\text { Cryo-duty } \\
\text { factor, } \%\end{array}$ & $\begin{array}{l}\text { Dynamic cryo- } \\
\text { loads per CM (W) }\end{array}$ \\
\hline SSR1 & 1.8 & 6.8 & 1.6 & 3.8 & 0.88 \\
\hline SSR2 & 2.9 & 9.9 & 5.2 & 5.3 & 2.8 \\
\hline LB650 & 2.8 & 9.7 & 7.3 & 5.2 & 4.0 \\
\hline HB650 & 2.8 & 9.8 & 19.2 & 5.2 & 10.3 \\
\hline Total* & & & 220 & & 130 \\
\hline
\end{tabular}

* This value includes contribution of HWR cryomodule operating in CW mode.

Table 2.9: Cooling power of the cryo-plant for low duty factor operation

\begin{tabular}{l|c|c|c}
\hline \hline Temperature of cooling circuit, $\mathrm{K}$ & 40 to 80 & 5 to 8 & 2 \\
\hline Cooling power, W & 4000 & 1500 & 550 \\
\hline \hline
\end{tabular}

Assuming pulsed operation one obtains the total dynamic cryo-loads at $2 \mathrm{~K}$ for all cryomodules to be $220 \mathrm{~W}$ for normal cavity discharge and $130 \mathrm{~W}$ for accelerated cavity discharge. Adding the static cryo-loads yields the total cryo-load at $2 \mathrm{~K}$ to be 366 and $275 \mathrm{~W}$, respectively. As shown in Table 2.9 the cooling power of the cryo-plant at $2 \mathrm{~K}$ is $550 \mathrm{~W}$. That leaves a margin of only about $50 \%$ for the less optimistic case of operation with normal cavity discharge. Such margin is considered being insufficient and therefore the accelerated cavity discharge is planned to be used. Note, that the total cryogenic heat load at $2 \mathrm{~K}$ is almost equally distributed between the static load and dynamic loads and is less than $15 \%$ of the cryogenic load for $\mathrm{CW}$ operation. The margin for cryo-plant cooling powers for the $5 \mathrm{~K}$ and $70 \mathrm{~K}$ circuits is close to a factor of two as can be seen from comparison of total static loads of Table 2.7 with the cryo-plant powers presented in Table 2.9. The Low $\mathrm{Q}_{0}$ program described in Section 3 is expected to decrease the dynamic cryo-losses and, consequently, significantly increase the margin for $2 \mathrm{~K}$ circuit.

Table 2.10: Maximum allowed heat loads per cryomodule

\begin{tabular}{l|c|c|c}
\hline \hline CM type & $\mathbf{7 0 ~ K}$ & $\mathbf{5 ~ K}$ & 2 K \\
\hline HWR & $250 \mathrm{~W}$ & $80 \mathrm{~W}$ & $50 \mathrm{~W}$ \\
\hline SSR1 & $250 \mathrm{~W}$ & $80 \mathrm{~W}$ & $50 \mathrm{~W}$ \\
\hline SSR2 & $250 \mathrm{~W}$ & $80 \mathrm{~W}$ & $75 \mathrm{~W}$ \\
\hline LB650 & $100 \mathrm{~W}$ & $15 \mathrm{~W}$ & $100 \mathrm{~W}$ \\
\hline HB650 & $300 \mathrm{~W}$ & $25 \mathrm{~W}$ & $220 \mathrm{~W}$ \\
\hline \hline
\end{tabular}

In conclusion we note that the requirements for the maximum cooling power specified by the 
Functional Requirements Specifications (FRS) exceed the values of cryo-loads presented in Table 2.7. Table 2.10 summarizes the FRS requirements for maximum cooling power which have to be supported by cryomodule design.

\subsubsection{RF Power and Suppression of Microphonics}

The RF system has to support $2 \mathrm{~mA}$ beam delivered in a $0.55 \mathrm{~ms}$ pulse at $20 \mathrm{~Hz}$. The system is based on a single RF source driving each RF cavity, for a total of 113 separate RF sources for SC cavities. It is anticipated that the amplifiers in the 162.5 and $350 \mathrm{MHz}$ sections will be solid state, while those in the $650 \mathrm{MHz}$ sections will be either inductive output tubes (IOTs) or based on the phase-locked magnetrons.

The average RF power delivered to the cavities consists of two contributions: 1) the energy transferred to the beam, and 2) the energy required to fill and discharge the accelerating cavities. The second contribution is about ten times larger than the first and, in general, the average power associated with this contribution does not depend on the peak RF power. For a fixed average power the RF cost increases with peak power and therefore the RF cost minimum is achieved with RF power equal to that required to accelerate the beam. Adopting this strategy yields a duty factor for the RF power amplifiers of about $9 \%$ for operation with the normal cavity discharge and about $13 \%$ for operation with the accelerated cavity discharge. One consequence of this strategy is that the cost savings associated with the pulsed power amplifiers in going from $\mathrm{CW}$ to low duty factor is modest and therefore $\mathrm{CW}$ capable RF amplifiers are planned. The RF requirements are summarized in Table 2.11. To estimate the peak RF power we assume that the maximum cavity detuning due to microphonics, $\delta f$, is equal to $20 \mathrm{~Hz}$ for all cryomodules [31]. That sets the optimal coupling,

$$
\beta_{c}=\sqrt{\left(1+\frac{I_{b} \cos \phi_{a}(R / Q) Q_{0}}{U_{0}}\right)^{2}+\left(\frac{2 \delta f Q_{0}}{f_{0}}\right)^{2}},
$$

the corresponding cavity bandwidth,

$$
\Delta f=\frac{f_{0}}{Q_{0}}\left(1+\beta_{c}\right)
$$

and the peak RF power,

$$
P_{\max }=\frac{U_{0}^{2}\left(1+\beta_{c}\right)^{2}}{4 \beta_{c}(R / Q) Q_{0}}\left(\left(1+\frac{I_{b} \cos \phi_{a}(R / Q) Q_{0}}{U_{0}\left(1+\beta_{c}\right)}\right)^{2}+\left(\frac{2 \delta f Q_{0}}{f_{0}\left(1+\beta_{c}\right)}\right)^{2}\right) .
$$

Here $I_{b}$ is the beam current, $U_{0}$ is the cavity voltage amplitude, and $\phi_{a}$ is the accelerating phase assumed to be equal to zero in these estimates.

The peak RF power presented in the last column of Table 2.11 sets the requirements on the power of RF power sources. It accounts for power loss in transmission (implying cable for 325 $\mathrm{MHz}$ and wave-guide for $650 \mathrm{MHz}$ ) and the power margin required for effective operation of voltage control system (low-level RF).

The large values of accelerating gradient and the comparatively small beam current determine a small cavity bandwidth and, consequently, high sensitivity of cavity detuning to microphonics and Lorentz Force Detuning (LFD). The major sources of cavity detuning are:

- Variations in the pressure of the surrounding helium bath, 
- Mechanical vibrations driven by external sources, and

- Radiation pressure on the walls from the electromagnetic field inside the cavity due to Lorentz force (Lorentz Force Detuning).

Table 2.11: Requirements for RF power ${ }^{*}$

\begin{tabular}{l|c|c|c|c|c|c}
\hline \hline $\begin{array}{l}\text { CM } \\
\text { type }\end{array}$ & $\begin{array}{c}\text { Power trans- } \\
\text { ferred to beam } \\
\text { per cav. }(\mathrm{kW})\end{array}$ & $\begin{array}{c}\text { Microphonics } \\
\text { amplitude } \\
(\mathrm{Hz})\end{array}$ & $\begin{array}{c}\text { Cavity half- } \\
\text { bandwidth, } \\
f / 2 Q_{L},(\mathrm{~Hz})\end{array}$ & $\begin{array}{c}\text { Power transfer } \\
\text { efficiency }\end{array}$ & $\begin{array}{c}\text { Power } \\
\text { margin }\end{array}$ & $\begin{array}{c}\text { Peak RF } \\
\text { power per } \\
\text { cavity }(\mathrm{kW})\end{array}$ \\
\hline HWR & 4 & 20 & 33 & $90 \%$ & $80 \%$ & 6.5 \\
\hline SSR1 & 4.1 & 20 & 43 & $90 \%$ & $80 \%$ & 6.1 \\
\hline SSR2 & 10 & 20 & 28 & $90 \%$ & $80 \%$ & 17 \\
\hline LB 650 & 23.8 & 20 & 29 & $94 \%$ & $80 \%$ & 38 \\
\hline HB 650 & 39.8 & 20 & 29 & $94 \%$ & $80 \%$ & 64 \\
\hline
\end{tabular}

As can be seen from Eq. (2.7) the power required to maintain a constant accelerating gradient in a detuned cavity rises rapidly as the cavity detunes. Providing sufficient reserve power to drive detuned cavities increases both the capital and the operating costs of an accelerator. If sufficient reserve is not available, the beam may be lost. Thus, all measures minimizing cavity detuning needs to be taken to keep RF power at a reasonable level. The measures can be separated into two broad categories: passive compensation and active compensation.

Passive compensation involves designing the machine and its components to minimize cavity detuning. In particular, the following design objectives are aimed:

- Minimization of the sensitivity of the cavity resonance frequency to variations in the helium bath pressure;

- The cryogenic system design has to minimize pressure variations in the $2 \mathrm{~K}$ helium bath; the target value is below 0.1 mbar for rms fluctuations and 1 mbar for maximum pressure deviation;

- The cryomodule design has to minimize transmission of external vibrations to the cavities;

- The civil engineering has to minimize vibrations in the tunnel and the transfer of these vibrations to cryomodules. In particular, large compressors have to be well isolated from ground and be located far enough from the tunnel.

Active compensation involves sensing cavity detuning and using feed-forward or feed-back to drive an actuator to compensate detuning in real-time. The detuning of each cavity can be determined in real-time from the base-band forward, reflected, and probe RF signals and used to drive a piezo actuator in a combination of adaptive feed-forward and feed-back loops.

Table 2.12 presents requirements to cavity detuning due to helium pressure variations [31], estimates for Lorentz force detuning and recently measured values for the HWR and SSR1 cavities. Measurements for SSR1 were done with a dummy tuner installed. As one can see the LFD detuning exceeds the cavity bandwidth by about one order of magnitude. That determines that 
operation in the pulsed regime is impossible without active frequency control with fast piezo-based tuners. Note that the HWR has no piezo tuner, and thus, cannot be used in a pulsed regime. As it was already stated the cryo-load in the HWR cryo-module is negligible compared to the total cryoload and the HWR will always operate with CW RF.

Table 2.12: Functional requirement specifications on cavity detuning due to helium pressure variations and Lorentz force detuning (LFD)

\begin{tabular}{r|c|c|c|c|c}
\hline \hline CM type & HWR & SSR1 & SSR2 & LB650 & HB650 \\
\hline Sensitivity to He pressure (FRS), $d f / d P, \mathrm{~Hz} /$ Torr & $<25$ & $<25$ & $<25$ & $<25$ & $<25$ \\
\hline$\ldots$. (measurements), $d f / d P, \mathrm{~Hz} / \mathrm{Torr}$ & 13 & 4.0 & - & - & - \\
\hline Estimated LFD sensitivity, $d f / d E^{2}, \mathrm{~Hz} /(\mathrm{MV} / \mathrm{m})^{2}$ & - & -5.0 & - & -0.8 & -0.5 \\
\hline$\ldots$ (measurements), $d f / d E^{2}, \mathrm{~Hz} /(\mathrm{MV} / \mathrm{m})^{2}$ & $-1.5^{*}$ & -4.4 & - & - & - \\
\hline Estimated LFD at nominal voltage (FRS), Hz & - & -500 & - & -192 & -136 \\
\hline$\ldots$ (measurements ) at nominal voltage, Hz & -122.4 & -440 & - & - & - \\
\hline \hline
\end{tabular}

Two cavities were measured in a test stand. The results are: -1.82 and $-1.3 \mathrm{~Hz} /(\mathrm{MV} / \mathrm{m})^{2}$. 


\subsubsection{Beam Dynamics in the SC Linac}

The high efficiency multi-turn injection to the Booster requires small transverse emittance of the injected beam. That determines quite strict requirements to the beam emittance. The rms normalized beam emittance budget for the SC Linac has been established at $0.15 \mathrm{~mm}-\mathrm{mrad}$ at the ion source and $0.3 \mathrm{~mm}$-mrad at the linac exit linac. The lattice design and the beam dynamics optimization are obtained using the TRACK, TraceWin and GenLinWin codes, which accurately account for effects of beam space charge making profound influence on the particle motion. A considerable effort was carried to benchmark the codes and ensure that they produce reliable calculations.
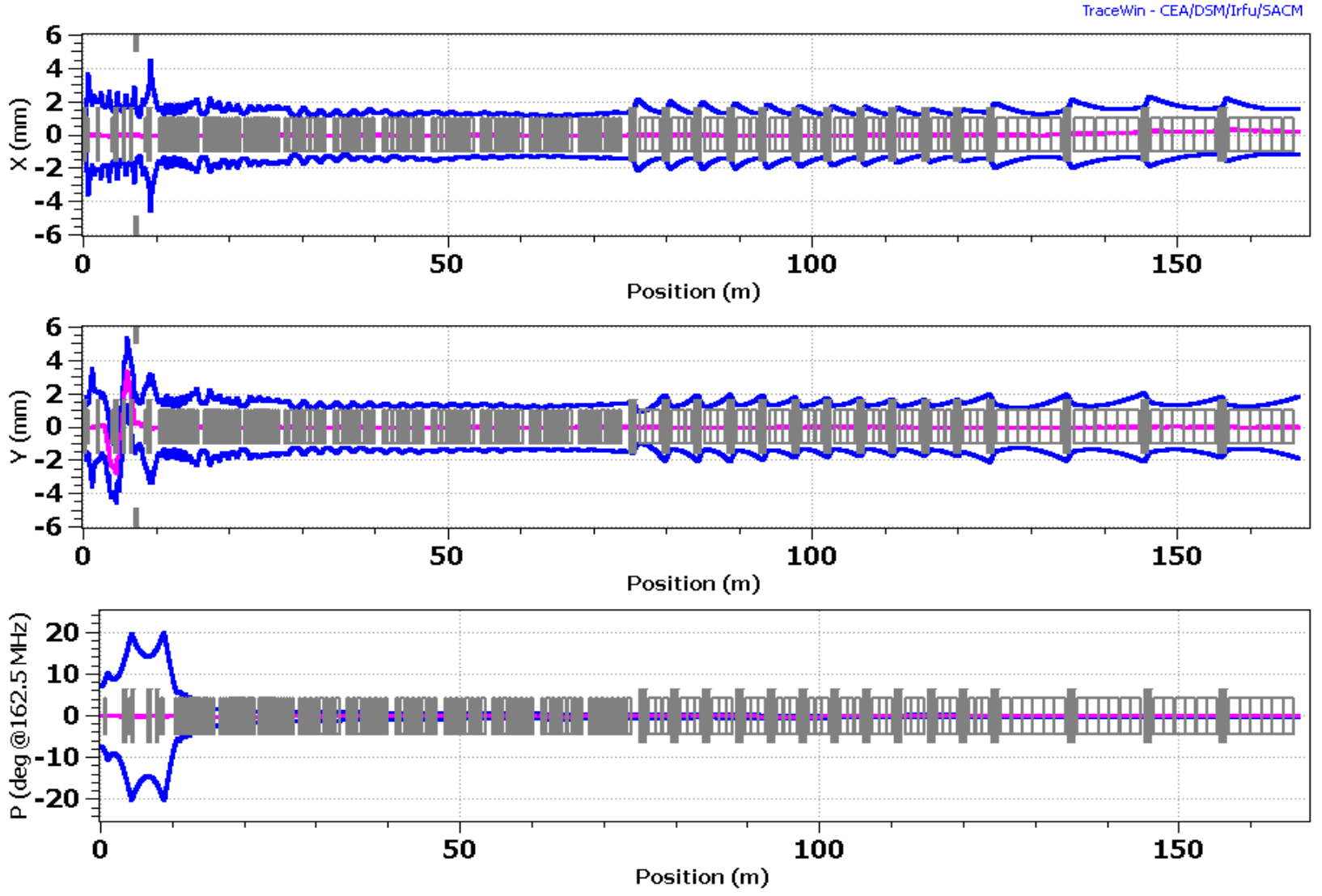

Figure 2.18: Horizontal (top) and vertical (center)) rms bunch envelopes and rms bunch length (bottom) along entire linac (from beginning of MEBT to the end of $0.8 \mathrm{GeV}$ linac); bunch population corresponds to the RFQ beam current of $5 \mathrm{~mA}$. Magenta lines show displacements of bunch centroid.

Figures 2.18 and 2.19 present the evolution of $1 \sigma$ beam envelopes and the corresponding rms longitudinal and transverse normalized emittances through the entire SC Linac. As one can see there is an emittance growth at the linac beginning where the space charge effects are large. Their effect diminishes with energy. Simulations show considerable margin in the value of transverse emittance, which is critically important for multi-turn strip injection into Booster. Figure 2.20 presents the phase space density of a bunch at the linac end. As one can see there are no significant distortions in the bunch phase space. Simulations show that there are no particles beyond $\sim 6 \sigma$. That result is supported by measurements performed at the SNS which has a bunch brightness similar to what is expected in the PIP-II. As in the SNS, the intrabeam stripping $[32,32]$ is expected to be the 
major source of particle loss. Figure 2.21 shows the beam loss power density due to intrabeam stripping. As one can see the losses due to this mechanism are below $0.1 \mathrm{~W} / \mathrm{m}$ everywhere even for $\mathrm{CW}$ operation. Figures $2.22-24$ show the strengths of focusing elements, the accelerating voltage and its phase. Finally, Figure 2.25 presents the beam energy along the linac, and Figure 2.26 presents the beta-functions obtained from the rms beam sizes and emittances, and hence describes the beam transport with the beam space charge forces included.

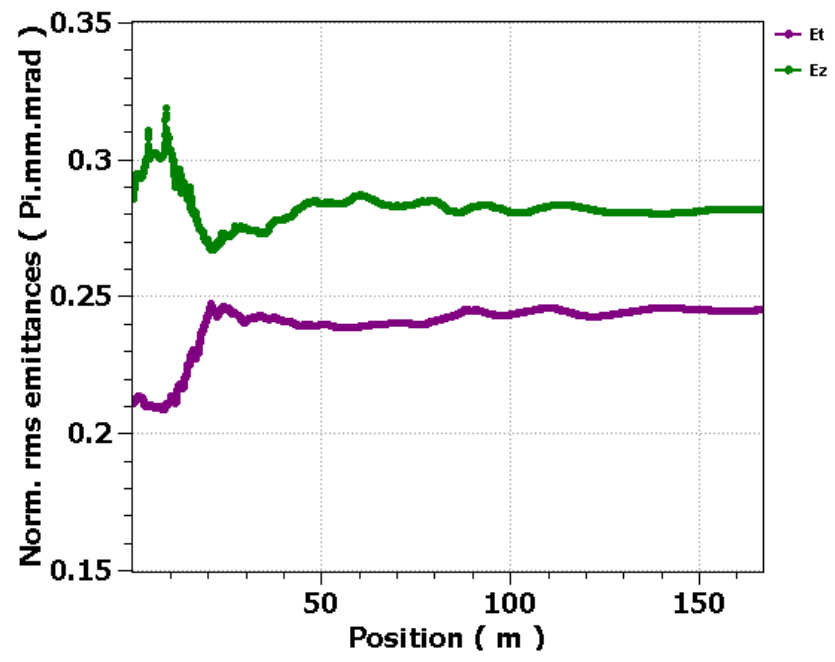

Figure 2.19: Rms normalized transverse (magenta, $\varepsilon_{x}=\varepsilon_{y}$ ) and longitudinal (green) emittances along the linac (from the RFQ exit to the linac end); the beam optics is the same as for Figure 2.18.
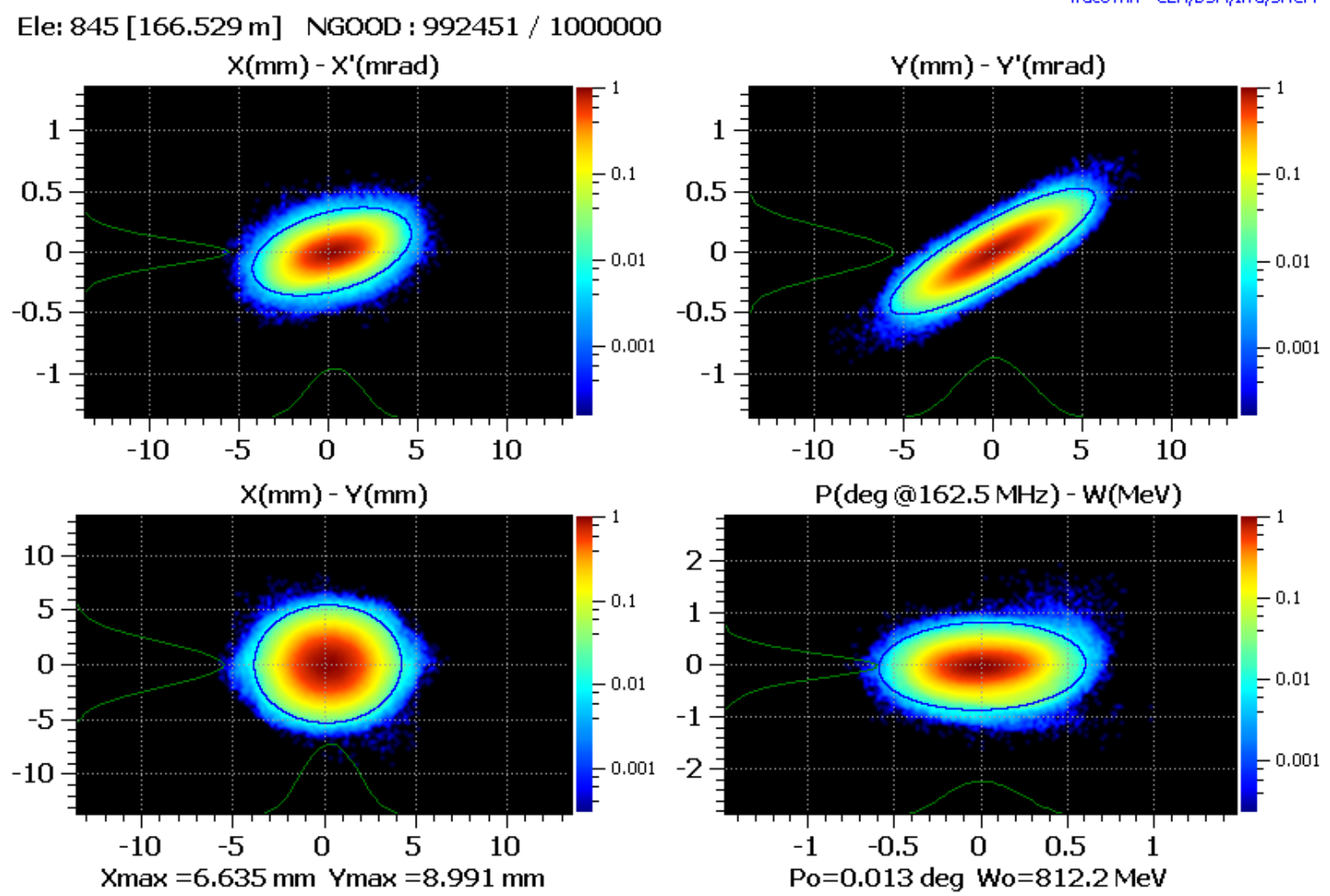

Figure 2.20: Phase space density of a bunch at the linac end; the beam optics is the same as for Figure 2.18. 


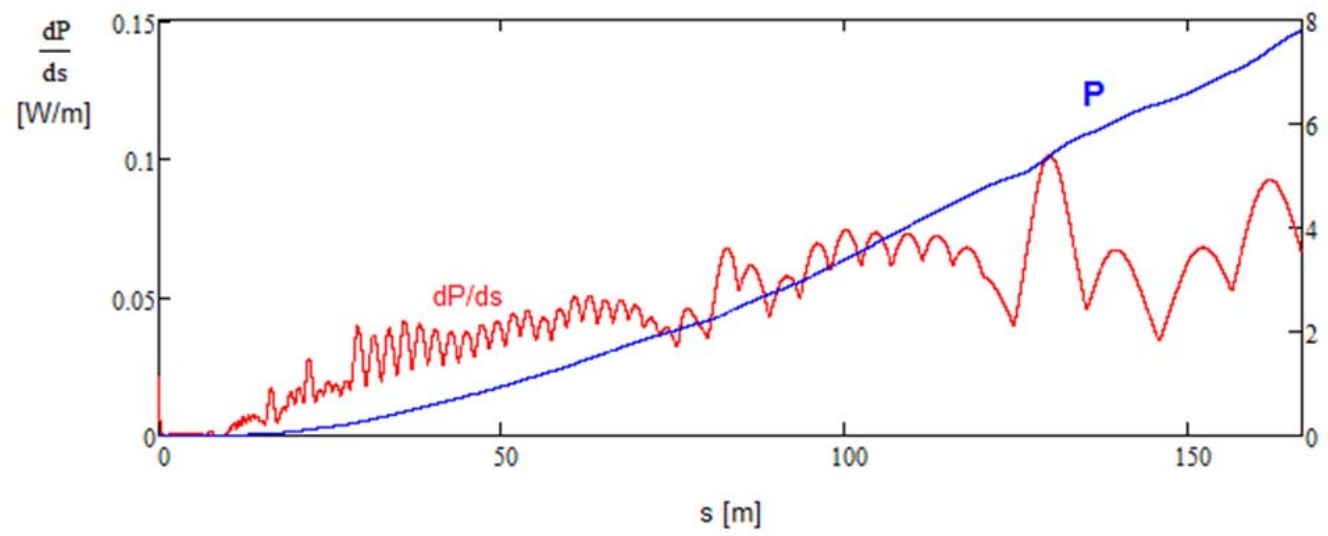

[W]

Figure 2.21: The beam power loss per unit length caused by intra-beam stripping (red) and its value integrated along the linac (blue); RFQ beam current $5 \mathrm{~mA}$, CW beam of $2 \mathrm{~mA}$ in the SC Linac (60\% of bunches are chopped off).
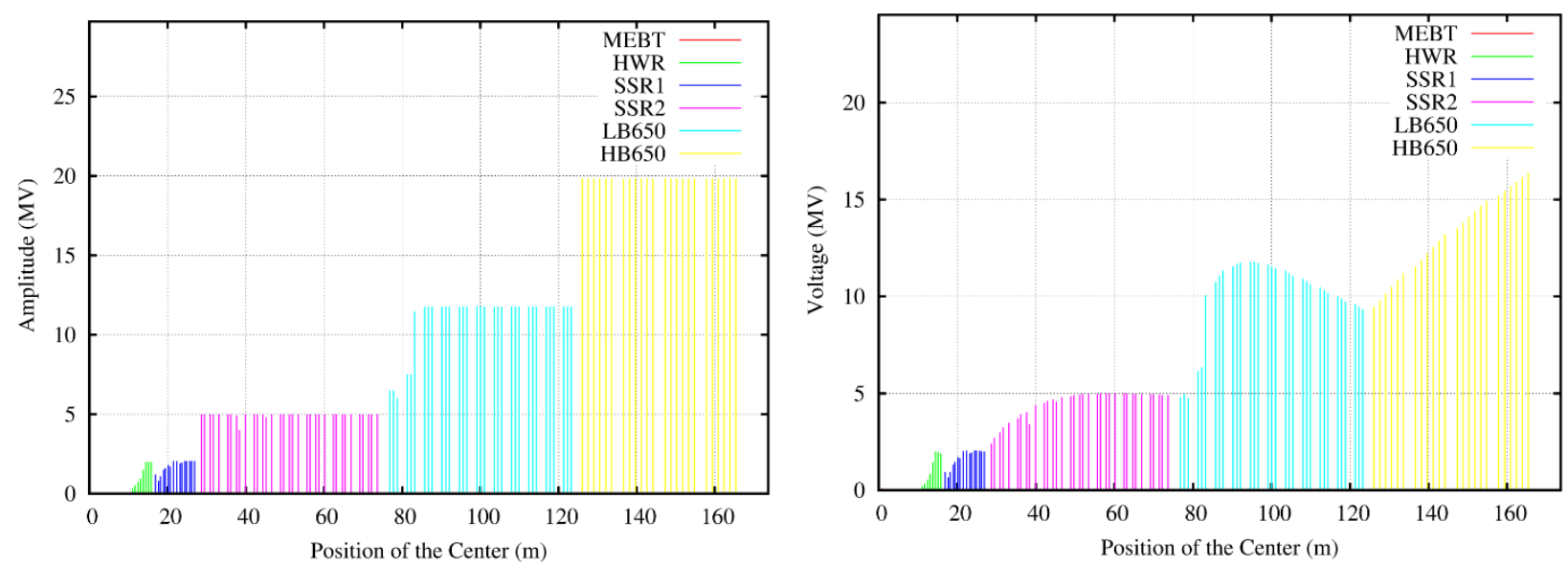

Figure 2.22: Accelerating voltage per cavity along the SC Linac corresponding to the beam optics presented in Figures 2.18 and 2.19; left - the voltage amplitude at the optimal beta, right - the voltage amplitude with the transit-time factors accounted.
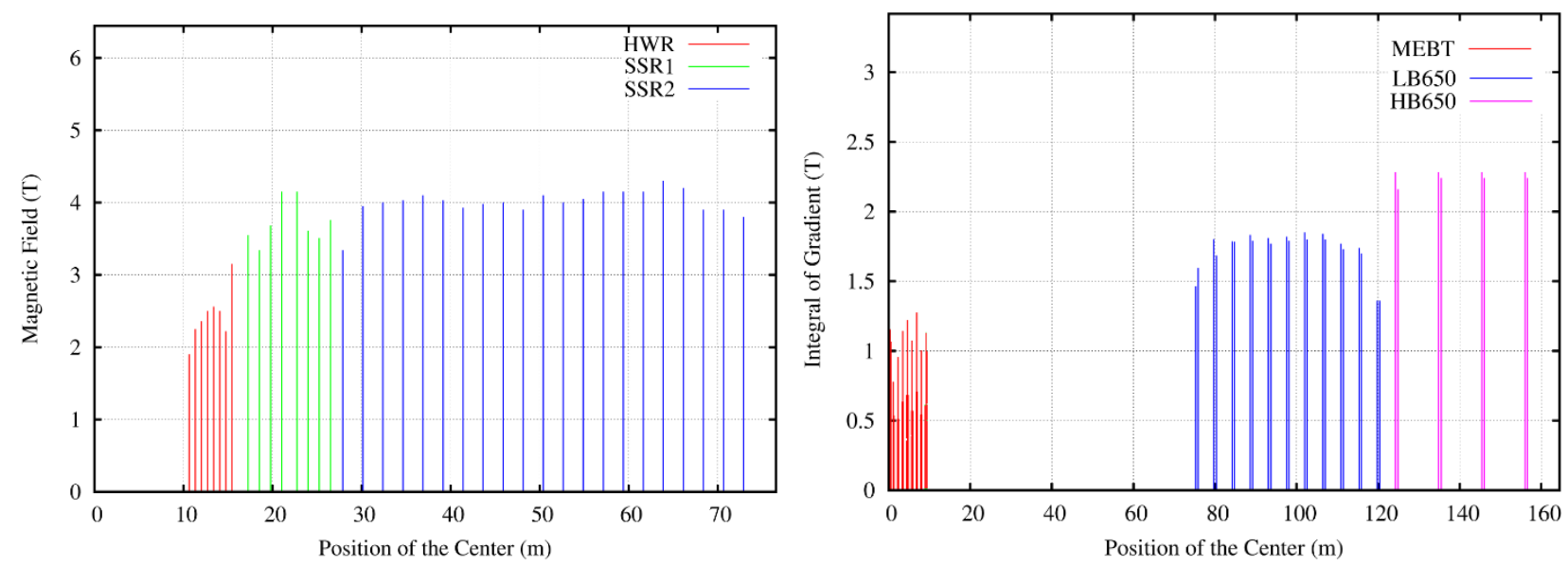

Figure 2.23: Magnetic field of focusing solenoids (left) and integral strength of quadrupoles (right) along the linac corresponding to the beam optics presented in Figures 2.18 and 2.19. 


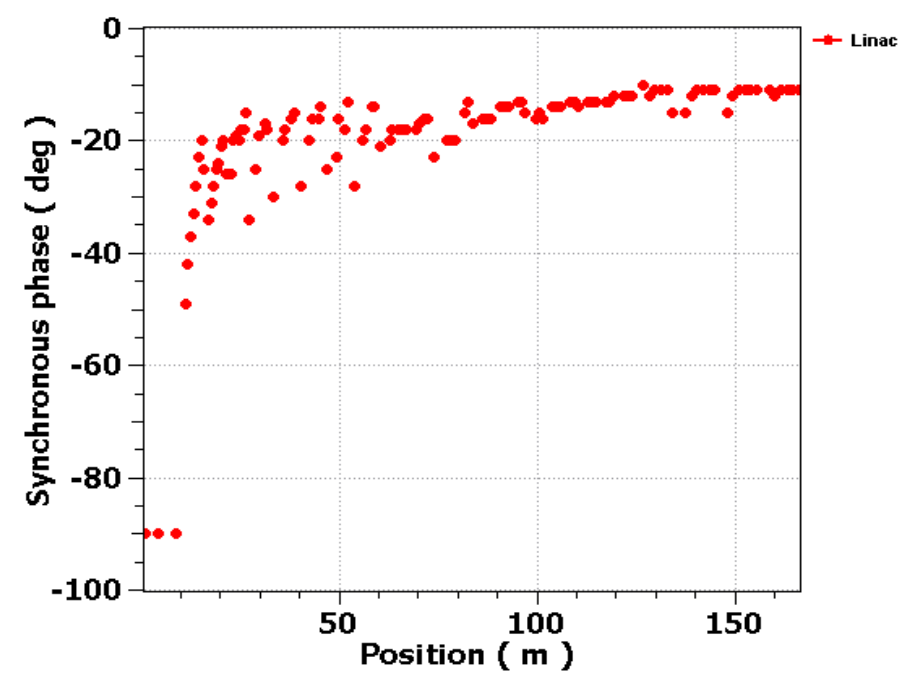

Figure 2.24: Accelerating phase along the linac corresponding to the beam acceleration presented in Figures 2.18 and 2.19. The first three dots belong to the MEBT bunching cavities which do not produce acceleration.

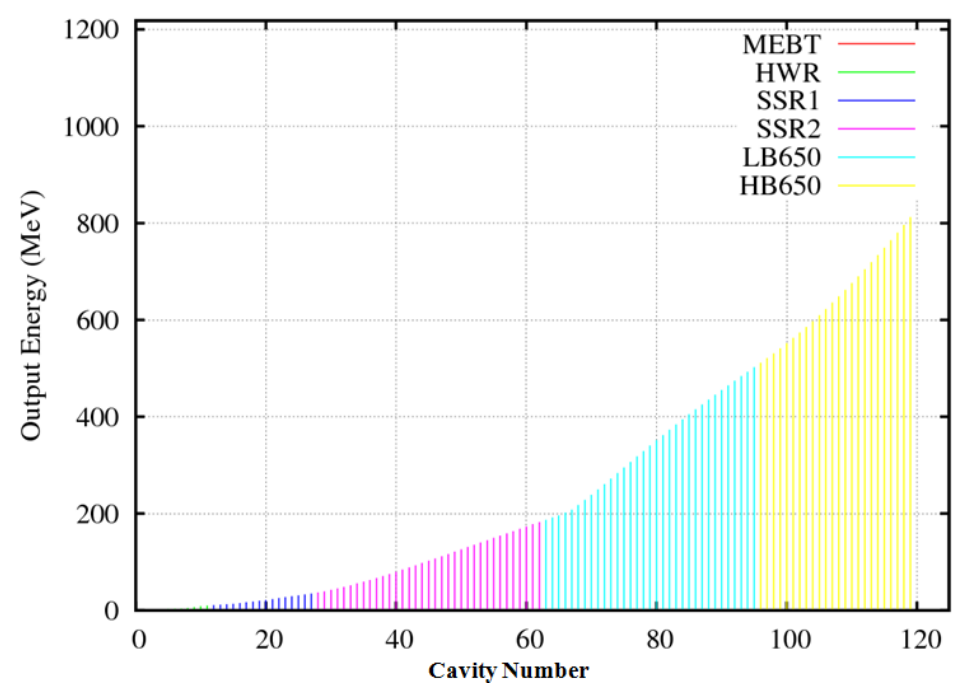

Figure 2.25: The beam energy along the linac.

As one can see from Figure 2.18 the transverse rms beam sizes change comparatively little along the linac, and their values do not exceed $3 \mathrm{~mm}$; i.e. the beam size reduction due to adiabatic damping with beam acceleration is compensated by corresponding increase in the beta-functions. Figure 2.27 presents the beam density projection to the $x$-plane and the aperture limitations along the linac. For the HWR, SSR 1 and SSR2 cryomodules the aperture is limited by apertures in the SC cavities of 33, 30 and $40 \mathrm{~mm}$, respectively. For the LB650 and HB650 cryomodules the cavity apertures are 83 and $118 \mathrm{~mm}$, correspondingly. They are sufficiently large and the aperture limitations are determined by the aperture of the vacuum pipe in the quadrupoles of $46 \mathrm{~mm}$ (standard 2" pipe). As stated above, in the case of a well-tuned machine the intra-beam stripping represents the main source of beam loss. To avoid the beam loss to cryogenic surfaces fixed aperture beam collimators are installed between each cryomodule for HWR, SSR1 and SSR2. Apertures in the collimators are chosen to be $5 \mathrm{~mm}$ smaller than the apertures of the downstream cryomodules. Their thickness increases with energy, reaching $4 \mathrm{~cm}$ of steel at the end of SSR2 
section. There are no dedicated collimators in the LB650 and HB650 sections. As in the SNS, the vacuum chambers in the quadrupoles will perform this role. Taking into account the comparatively small beam loss it appears unnecessary to have additional radiation shielding around the collimators which otherwise would require quite a large amount of shielding material.

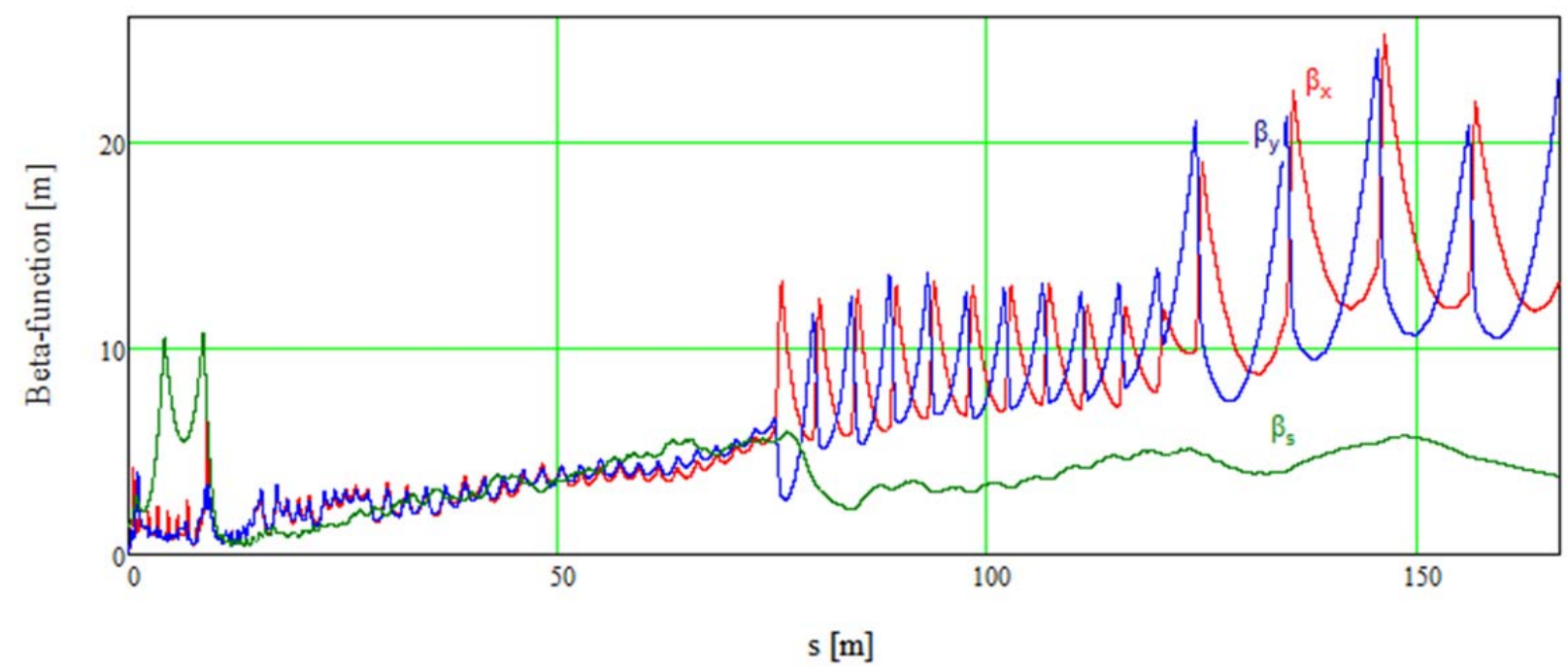

Figure 2.26: The beta-functions for $\mathrm{x}, \mathrm{y}$ and s planes. The values were computed from rms beam sizes and emittances obtained by beam tracking with TraceWin for $5 \mathrm{~mA}$ RFQ current.

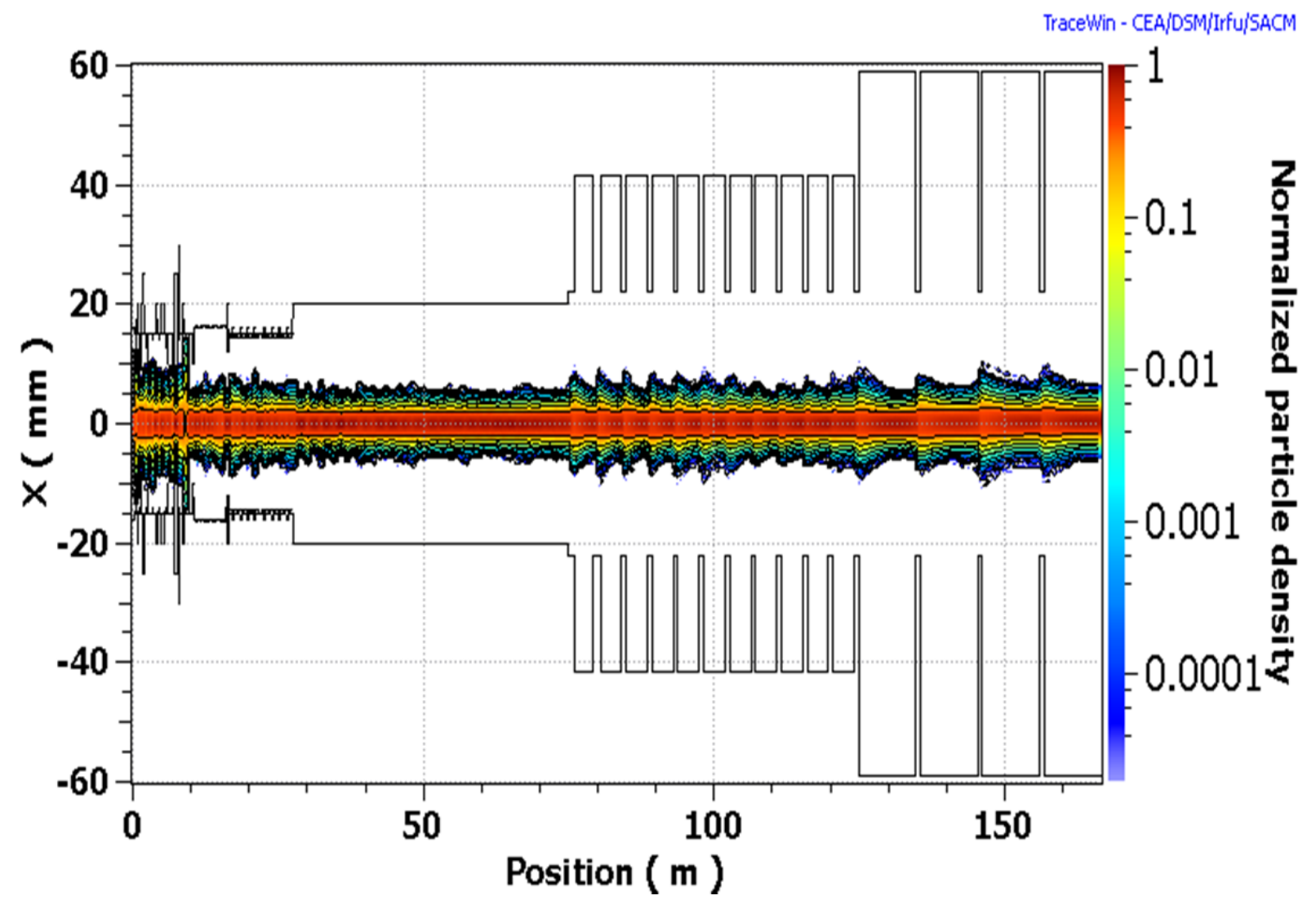

Figure 2.27: Beam density projection to the $x$-plane and aperture limitations along the linac from RFQ exit to the linac end. 

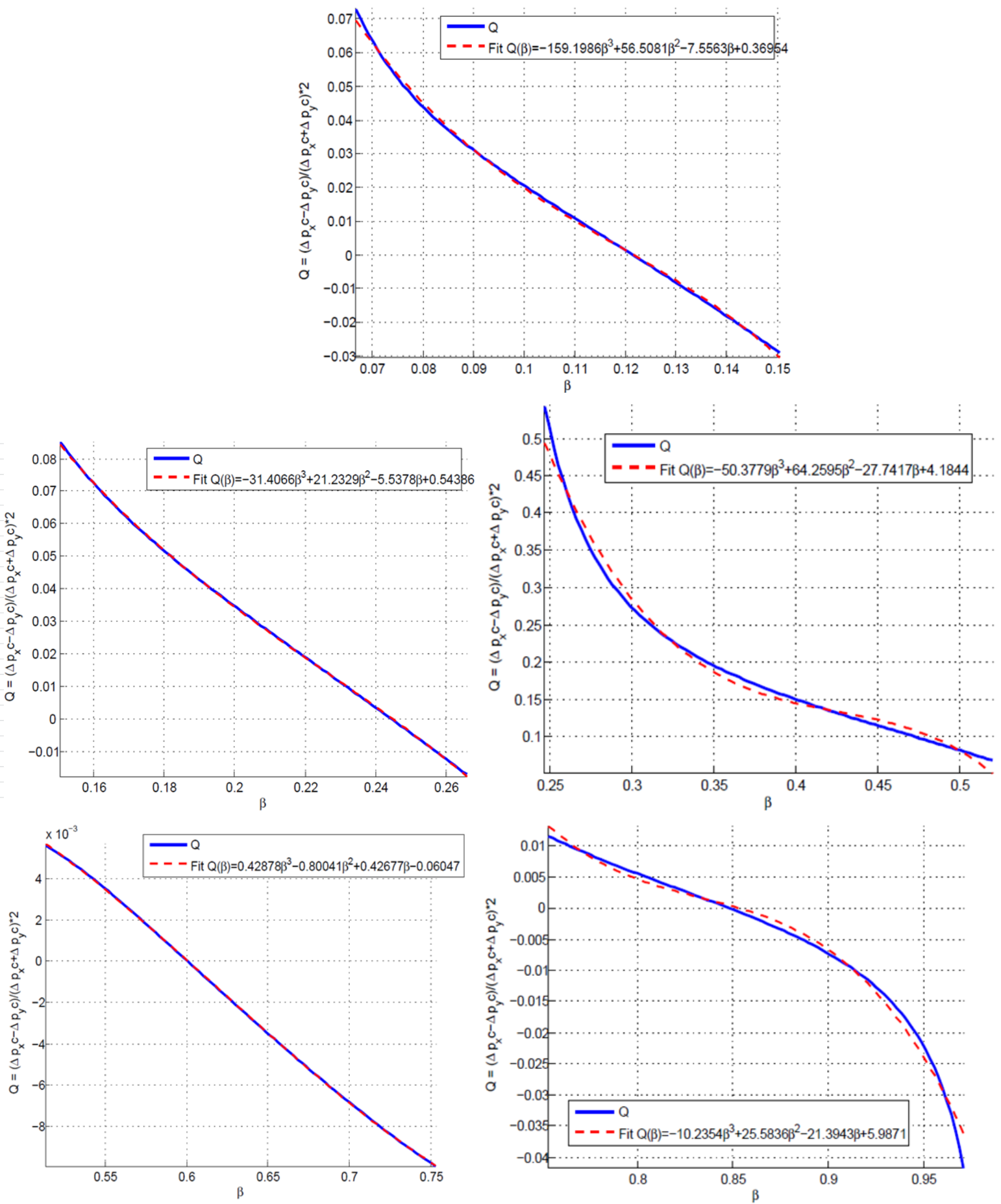

Figure 2.28. Ratio of quadrupole focusing to axial symmetric focusing for HWR (top), SSR1 (middle-left), SSR2 (middle-right), LB650 (bottom-left) and HB650 (bottom-right) versus the particle velocity $\beta$ in the operating domain; blue and red lines present simulation results and a polinomial fit, respectively.

The HWR, SSR1 and SSR2 cavities are not axially symmetric. Therefore their quadrupole components cannot be compensated over the entire range of cavity operation. Figure 2.28 presents the dependence of the quadrupole effect on the beam velocity. Numeric simulations verified that in 
the range of PIP-II parameters the strength of quadrupole field is proportional to the strength of axially-symmetric cavity defocusing and, consequently, is proportional to the sine of accelerating phase. That determined the choice of values presented in Figure 2.28. The stem in the HWR cavity is located in the horizontal plane and therefore this quadrupole field represents a normal quadrupole. Due to engineering limitations, mainly related to the RF couplers, the SSR1 and SSR2 cavities are rolled by $45^{\circ}$. Consequently, their quadrupole field is also rolled and is equivalent to a skew-quadrupole field. The cavity quadrupole and skew-quadrupole fields have comparatively small effect on the beam dynamics. To compensate these fields the SSR1 and SSR2 cryomodules will have skew-quadrupole correction coils located inside focusing solenoids. The skewquadrupole field will be created by a misbalance in the currents of independently powered coils of $x$ - and $y$-dipole correctors. Note that the focusing solenoids located in the HWR, SSR1 and SSR2 cryomodules rotate the plane of betatron motion. That allows one to improve quality of compensation by choice of appropriate signs of the magnetic fields in solenoids. The HWR cryomodule has only 8 cavities which introduce acceptable coupling between $x$ - and $y$-planes. Therefore it does not have coupling correction.

The quadrupole fields in LB650 and HB650 cavities are related to the RF couplers and compared to the HWR, SSR1 and SSR2 are significantly smaller as can be seen in the bottom row of Figure 2.28. Quadrupole fields of the elliptic cryomodules will be corrected by the main focusing quadrupoles located between cryomodules. The RF couplers also create dipole fields resulting in dipole kicks which values are also dependent on the beam velocity. The peak values of the kick, $\Delta \mathrm{p}_{\perp} \mathrm{c}$, are about $3.6 \mathrm{keV}$ for LB650 and $2.6 \mathrm{keV}$ for HB650 cavities. These values are small and are not expected to produce any visible effect on the beam motion in the linac. 


\subsection{Linac-to-Booster Beam Transport}

\subsubsection{Particle Loss and Limitations on Beam Transport Parameters}

Low loss beam transport is critical in the operation of a MW class facility. The $\mathrm{H}^{-}$transport should have sufficiently small loss to minimize residual radiation in the tunnel. It is highly desirable to keep the residual radiation level below $15 \mathrm{mrem} / \mathrm{hr}$ at $30 \mathrm{~cm}$ from component surface. Many facilities use the metric of $1 \mathrm{~W} / \mathrm{m}$ as a limit for "hands on" maintenance; however, at energies about or above $1 \mathrm{GeV}$, a $1 \mathrm{~W} / \mathrm{m}$ loss rate produces a peak contact residual dose rate of $\sim 150 \mathrm{mrem} / \mathrm{hr}$ at $30 \mathrm{~cm}$ on a bare beam pipe [34]. Although magnets shield the radiation and significantly reduce the residual activation on their external surfaces the radiation of unshielded pieces including magnet interfaces and instrumentation locations has to be sufficiently small. That sets the maximum acceptable loss rate. These levels are based upon MARS calculations and used for order of magnitude estimations. A more accurate estimation will be required once a detailed model of the transport line is available. Setting a desirable activation level to $15 \mathrm{mrem} / \mathrm{hr}$ at $30 \mathrm{~cm}$ results in a loss goal of $\sim 0.1 \mathrm{~W} / \mathrm{m}$ at energies about or above $1 \mathrm{GeV}$. Initial linac operation will be in a short-pulse regime where the total beam power is about $18 \mathrm{~kW}$. Consequently, a fractional loss rate of $5 \times 10^{-6} \mathrm{~m}^{-1}$ is required. Future $\mathrm{CW}$ operation will require a fractional loss rate limit of about $5 \times 10^{-8} \mathrm{~m}^{-1}$.

The primary loss mechanisms for $0.8 \mathrm{GeV}$ transport are the $\mathrm{H}^{-}$intra-beam stripping, Lorentz stripping, inelastic beam-gas scattering, and scraping of beam halo on the apertures. Stripping due to black-body radiation inside the room temperature beam pipe is negligible at this energy.

As shown in Figure 2.21 the intra-beam stripping in the linac results in an acceptable loss rate even in the case of $\mathrm{CW}$ beam. The particle momentum spread results in natural debunching in the course of beam transport from the linac to the Booster, so that the bunch length increases by more than an order of magnitude (from $1.1 \mathrm{~mm}$ to about $14 \mathrm{~mm}$ ). The strength of transverse focusing in the beam line is similar to the focusing strength at the linac end. That leads to the reduction of intrabeam stripping in the same proportion resulting in its contribution at the level of $100 \mu \mathrm{W} / \mathrm{m}$ at the end of the transport line for $1 \%$ duty factor.

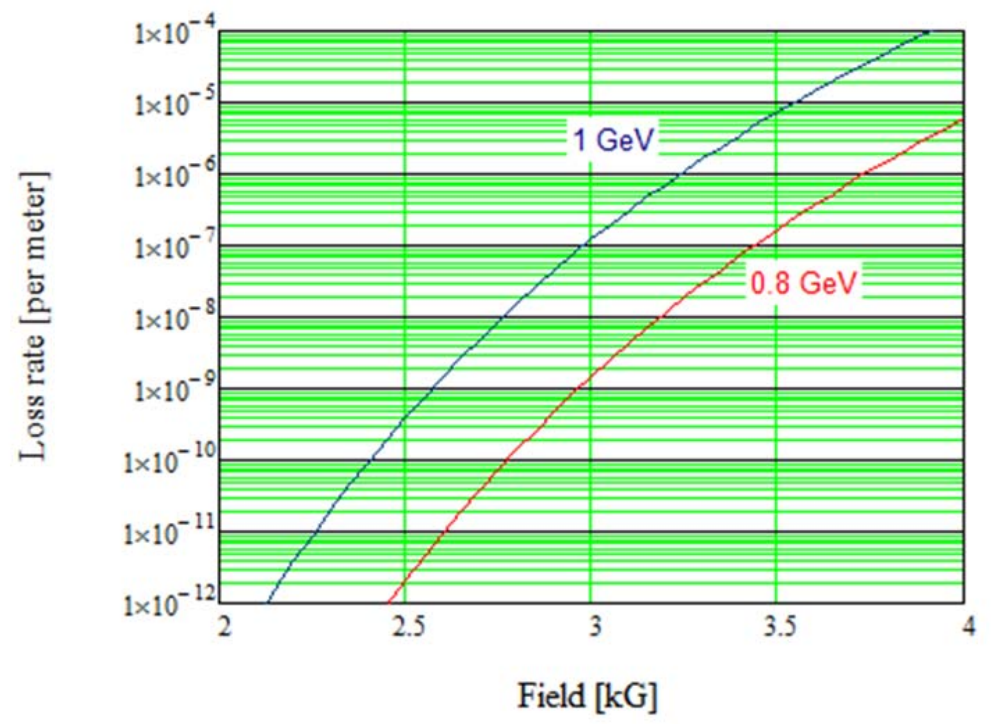

Figure 2.29: Fractional loss due to Lorentz stripping for 0.8 and $1 \mathrm{GeV} \mathrm{H}-$ beams traveling in a dipole field. 
Beam motion in a magnetic field excites an electric field in the beam frame. If this electric field is sufficiently strong, it can detach the weakly bound outer electron (Lorentz stripping) from $\mathrm{H}^{-}$ion. The results of experimental measurements for $\mathrm{H}^{-}$lifetime are presented in Ref. [35] and can be approximated by following equation:

$$
\tau_{E}(E)=\frac{A_{\tau}}{E} e^{B_{\tau} / E}
$$

where the constants are $A_{\tau}=2.47 \cdot 10^{-8} \mathrm{~s} \mathrm{~V} / \mathrm{cm}, B_{\tau}=44.94 \cdot 10^{6} \mathrm{~V} / \mathrm{cm}$. Figure 2.29 shows the corresponding loss rates per meter for a 0.8 and $1 \mathrm{GeV} \mathrm{H}^{-}$beams as a function of magnetic field. The bending radius in dipoles was chosen so that the loss rate would not exceed $10^{-8} \mathrm{~m}^{-1}$ if the 1 $\mathrm{GeV}$ beam send through the line. That results in magnetic field of $2.77 \mathrm{kG}$ and the bending radius of $20.697 \mathrm{~m}$. Scaling magnetic field to the $0.8 \mathrm{GeV}$ beam energy yields its value to be $2.36 \mathrm{kG}$ and a loss rate of $3 \cdot 10^{-13}$.

The loss rate due to $\mathrm{H}^{-}$scattering on residual gas molecules is proportional to their density and their ionization cross sections. The cross sections decrease proportionally to $\beta^{-2}$ and therefore are weakly dependent on energy for energies about or above $1 \mathrm{GeV}$. The cross section of $\mathrm{H}^{-}$stripping for $0.8 \mathrm{GeV}$ beam on residual gas is about $10^{-19} \mathrm{~cm}^{2}$ for atomic hydrogen [36] and grows approximately proportional to $Z$ for heavier atoms. The requirement of $10^{-8} \mathrm{~m}^{-1}$ for partial loss rate yields a vacuum requirement of $10^{-8}$ Torr or better for $\mathrm{H}_{2}$ and about an order of magnitude better for heavy molecules (hydrocarbons, water, etc.). Consequently, an application of vacuum practices developed in Fermilab for not-baked vacuum systems, which routinely achieve low $10^{-8}$ Torr, should be sufficient.

\subsubsection{Linac-to-Booster Transfer Line}

The Linac-to-Booster Transfer Line transfers the beam from the exit of the SC Linac to the injection magnet of the Booster. The linac and Booster will have the same elevation. Therefore the major part of the Transfer Line will be located at the same elevation with a vertical rise shortly before the Booster injection area.

The Booster beam plane elevation is $3.98 \mathrm{ft}$. $(1.213 \mathrm{~m})$ above the enclosure floor or at $722.5 \mathrm{ft}$. $(220.218 \mathrm{~m})$ above the sea level. The SC Linac beam plane elevation is planned to be $4.265 \mathrm{ft}$. $(1.300 \mathrm{~m})$ above the floor of the linac enclosure, consequently, the estimated elevation of the linac floor is $722.215 \mathrm{ft}$. $(220.131 \mathrm{~m})$. The Transfer Line and its enclosure elevation are planned to be the same as for the linac. The injection into the Booster will be vertical, with an injection C-magnet placed 13.2 in. $(33.4 \mathrm{~cm})$ above the Booster beam line. The direction of the SC Linac is directed at an angle of $-217 \mathrm{deg}$. with respect to the injection straight in the Booster, consequently, the total bending angle of the Transfer Line of $217 \mathrm{deg}$.

The momentum spread of the linac is sufficiently small. Therefore, RF debunching is not planned and, consequently, no debunching RF cavities are anticipated. To keep the dispersion and beta-functions comparable to their values in the SC Linac and the Booster, a FODO lattice with a total of 32 dipoles is chosen. Consequently, each dipole has to provide a bending angle of 6.781 deg. and be $2.45 \mathrm{~m}$ long.

To minimize the total length of the line the entire bend is split into two arcs. That also minimizes undesired intersections with existing tunnel enclosures, utility/communication corridors and cryo lines. The first arc drives the beam off the linac axis, away from the linac enclosure and the dump area. The second arc completes the bend. The two arcs are connected by a straight transport line. Figure 1.1 shows the PIP-II site layout which includes the SC Linac, the transfer line 
and Booster.

The lattice consists of FODO cells with 90 deg. phase advance per cell both horizontally and vertically. The cell lengths in the arcs and the straight section are equal. Such choice automatically assures that arcs and straight sections are matched to each other, and allows one to connect all quads serially and power them from a single power supply. The two arcs are composed of 4 and 12 cells, making both of them achromatic. The straight section downstream the first arc consists of 2 cells. The exact cell length is determined by the geometrical constraints of the accelerator complex and is equal to $12 \mathrm{~m}$. A low power collimator is installed in the center of the first arc where the maximum dispersion is achieved. It scrapes off-momentum particles, leaving a momentum window of about $\pm 1.5 \cdot 10^{-3}$. The length allocated for the collimator is about $1 \mathrm{~m}$. Since each arc cell includes 2 bends, the packing factor of the arcs is $41 \%$. This choice leaves sufficient space for horizontal and vertical correctors after each focusing and defocusing quadrupole, respectively, as well as for ion pumps, BPMs and other instrumentation. It also foresees space for a possible installation of debunching RF cavities and additional collimators if needed in the future. The integrated strength of the quadrupoles is fixed by the constraints on the phase advance per cell and results in about $0.24 \mathrm{~m}^{-1}\left(L=20 \mathrm{~cm}, G=0.58 \mathrm{kG} / \mathrm{cm}\right.$ at $\left.E_{k i n}=800 \mathrm{MeV}\right)$.

The vacuum chamber in the Transfer Line is manufactured from 1.5" stainless steel pipe. It has an aperture of $34 \mathrm{~mm}$ in diameter.

There is a matching section between the end of the linac and the first arc. It continues the periodic doublet lattice of the linac with the distance between the doublets equal to the period length in the HB650 section of the linac. Such choice allows an installation of four additional cryomodules in the future, so that the linac energy could be upgraded to about $1.2 \mathrm{GeV}$.

The last section of the Transfer Line starts downstream of the second arc and is aligned with the Booster injection straight. The injection will be in the vertical plane with a $\mathrm{C}$-magnet bending the beam downward as shown in Figure 2.30. To bring the beam to the elevation of the C-magnet a vertical dogleg is realized using 2 bending magnets of $1.8 \mathrm{~m}$ length and magnetic field of $2.5 \mathrm{kG}$. Quadrupoles are placed before the first vertical bend and a triplet is placed after the second bend to match the Twiss parameters at the entrance of the C-magnet.

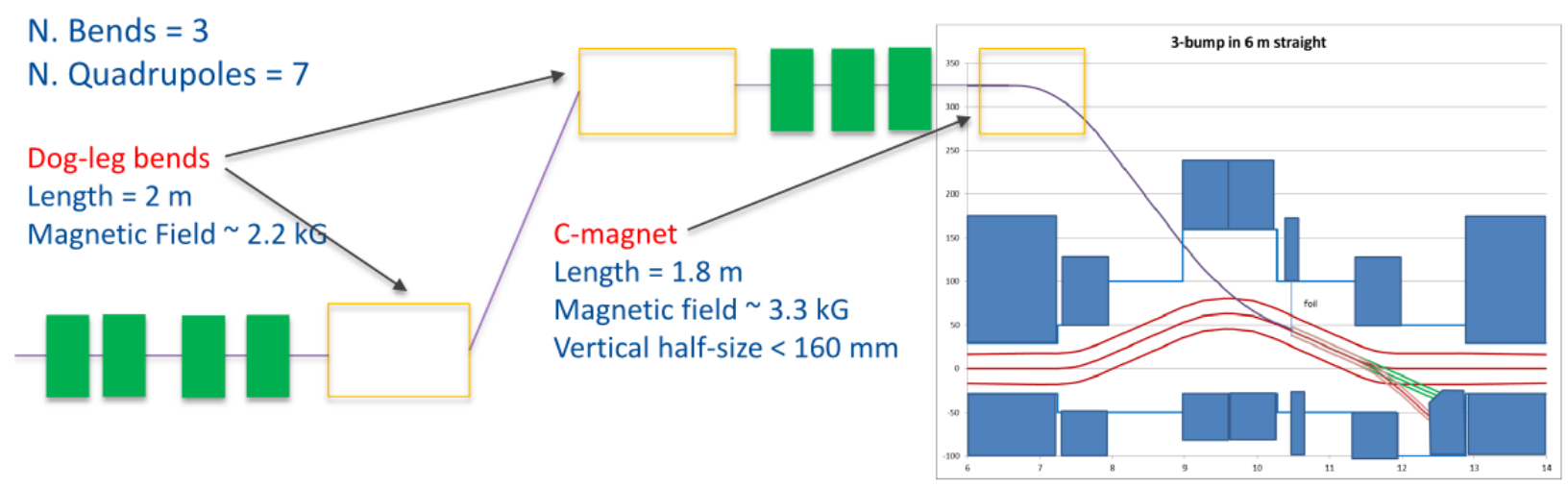

Figure 2.30: Schematic layout of the injection beam line.

The optical functions of the entire Transfer Line are presented in Figure 2.31. The maximum horizontal dispersion in the arcs is about $3.8 \mathrm{~m}$. The $\pm 1.5 \mathrm{~cm}$ aperture limitation in the momentum collimation corresponds to a $\pm 18 \sigma$ momentum aperture for the nominal rms momentum spread at the linac end $\left(\sigma_{p}=2 \cdot 10^{-4}\right)$. 


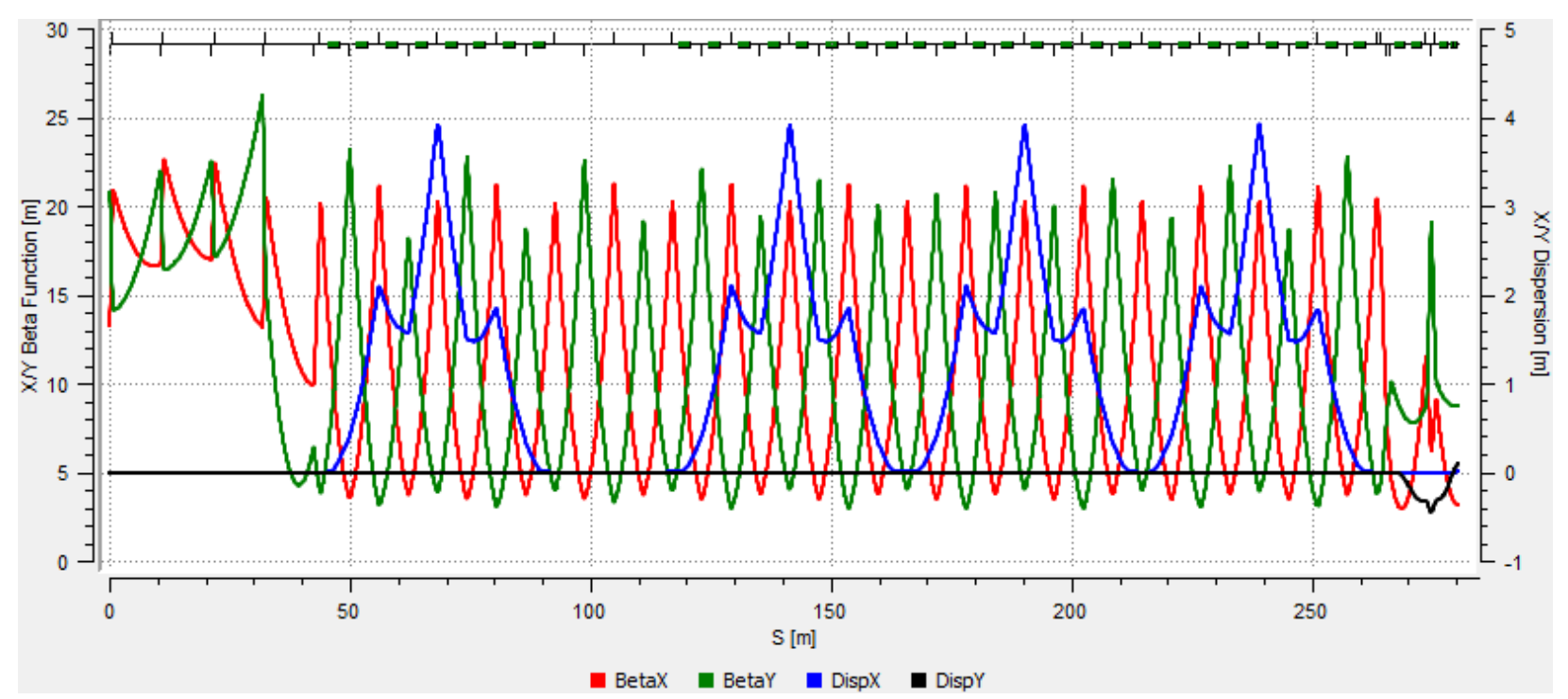

Figure 2.31: Optics of the Transfer Line from the SC linac end to the stripping target.

\subsubsection{Beam Based Linac Energy Stabilization}

As shown below a high quality injection to the Booster requires the energy stability better than $10^{-4}$. Present experience does not guarantee that such energy stability can be achieved if the beam energy is stabilized only by internal feedbacks separately stabilizing each cavity voltage. In this case the beam based energy stabilization is the only possibility to address the problem. Similar approach is used in the present Fermilab linac. The energy correction is applied to the cavities of the last SC cryomodule where the synchrotron motion is greatly slowed down and voltage variation will have little effect on beam dynamics. It is expected that such a system will improve the energy stability from the $10^{-3}$ level achieved with local feedbacks to the desired value of better than $10^{-4}$.

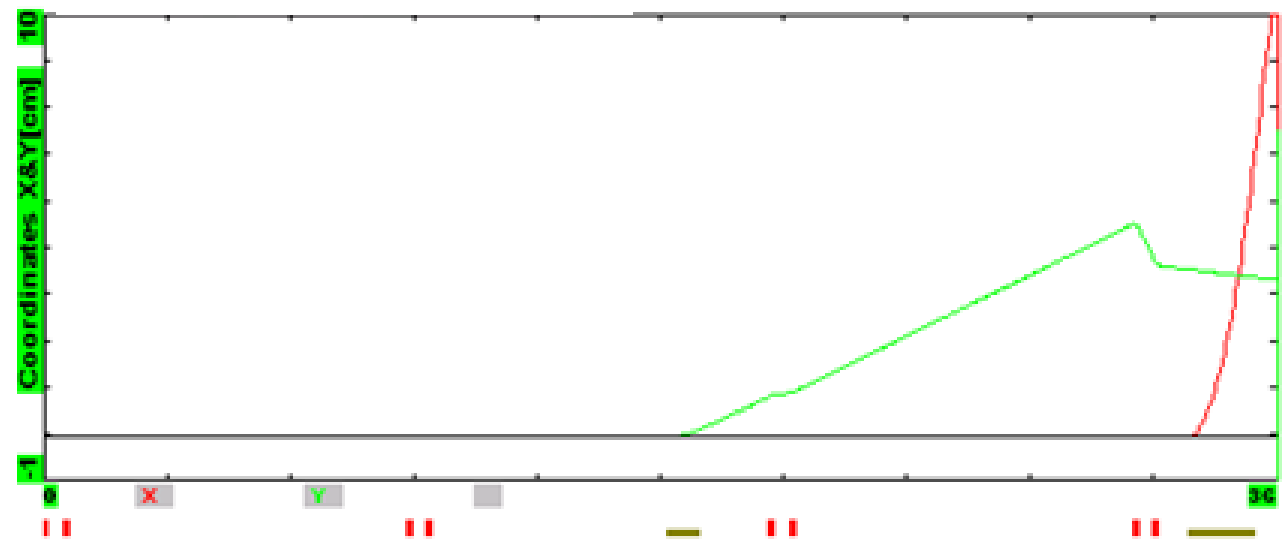

Figure 2.32: Transverse (vertical, green) beam displacement for the beam extracted to the beam dump in the straight line following the end of the last SC cryomodule. The red line presents the horizontal beam displacement due to field of the Lambertson septum. Longitudinal coordinate is the same as in Figure 2.31 .

To avoid unnecessary beam loss in the transfer line and the Booster the beam will be initially directed to the beam dump located at the linac end (see Figure 1.1). After the energy is stabilized to the required level $(10-20 \mu$ s later $)$ the beam is switched to the transport line and delivered to the Booster. The switching has to be done within $\sim 0.5 \mu$ s to avoid changes of accelerating voltage 
during switching time. It will be performed by a fast vertical kicker located at the end of the second empty cryomodule slot as shown in Figure 2.32. The beam extraction from the transport line to the beam dump will be performed by a Lambertson septum magnet located in the fourth empty cryomodule slot (the last slot before the arc). The extraction will be in the horizontal plane. The strength of the kicker and septum are 0.18 and $3.4 \mathrm{kG} \cdot \mathrm{m}$, respectively.

The beam energy will be measured by a time-of-flight system based on $2.6 \mathrm{GHz}$ resonant cavities excited by the beam. With a baseline of about $20 \mathrm{~m}$ it supports the required relative accuracy of energy measurement. The absolute energy measurement is not required.

An alternative choice for energy measurement can be based on the beam position measurements in the first arc of the Transport Line. Its $4 \mathrm{~m}$ dispersion supports the needed accuracy. Such a choice will require an additional beam dump in the straight transport line between the two arcs. This straight line has enough space for installation of the kicker and septum. 


\subsection{Booster Modifications}

\subsubsection{Technical Requirements and Scope}

The performance requirements of the Booster are summarized in Table 2.13. The $800 \mathrm{MeV}$ injection energy is selected to provide an increase in beam intensity of about $50 \%$ beyond current operations, accompanied by a 30\% decrease in the space-charge (Laslett) tune shift at injection. This choice is made to provide more efficient beam capture and acceleration, in order to minimize losses at the higher beam intensity required in PIP-II. The requirements on longitudinal beam emittance are set by slip-stacking in the Recycler.

Table 2.13: Performance requirements for the Booster

\begin{tabular}{l|c}
\hline \hline Performance Parameter & Requireme \\
\hline Particle Species & Protons \\
\hline Input (H') Beam Energy (Kinetic) & $800 \mathrm{MeV}$ \\
\hline Output Beam Energy (Kinetic) & 8.0 \\
\hline Protons per Pulse (injected) & $6.7 \times 10^{12}$ \\
\hline Acceleration efficiency & $97 \%$ \\
\hline Protons per Pulse (extracted) & $6.5 \times 10^{12}$ \\
\hline Beam Pulse Repetition Rate & $20 \mathrm{~Hz}$ \\
\hline RF Frequency (injection) & $44.7 \mathrm{MHz}$ \\
\hline RF Frequency (extraction) & $52.8 \mathrm{MHz}$ \\
\hline Total RF voltage & $1 \mathrm{MV}$ \\
\hline Injection Efficiency & $98 \%$ \\
\hline Injection Time & $0.55 \mathrm{~ms}$ \\
\hline Injection Turns & 292 \\
\hline Beam Emittance (95\%, normalized; $\left.\varepsilon_{x}=\varepsilon_{y}\right)$ & $16 \mu \mathrm{m}$ \\
\hline Maximum Laslett Tune Shift (space charge) & -0.17 \\
\hline Delivered Longitudinal Emittance $(97 \%)$ & $0.08 \mathrm{eV} \cdot \mathrm{s}$ \\
\hline Delivered Momentum Spread (97\% full height) & $12.2 \mathrm{MeV}$ \\
\hline Delivered Bunch Length (97\% full length) & $8.2 \mathrm{~ns}$ \\
\hline \hline
\end{tabular}

The primary areas that need to be addressed in order to reach the performance goals listed above are given in Table 2.14. Among these injection and beam quality are expected to present the primary challenges.

This section will describe concepts and approaches in the areas listed above. These descriptions assume successful completion of the PIP tasks currently underway. However, this discussion is preliminary and may change after more extensive investigations are completed. It is required that Booster beam losses be maintained at less-than-or-equal-to present levels. The current operating limit is 525 watts ring-wide, augmented by independently set beam loss 
monitor (BLM) trip points in each long and short straight section.

Table 2.14: Booster areas requiring consideration as part of PIP-II.

\begin{tabular}{l|l}
\hline \hline Topic & Associated Items \\
\hline Injection & Injection girder and loss control \\
\hline Capture & RF capture, timing and emittance control \\
\hline Acceleration and Transition & Loss control, RF requirements and transition control \\
\hline Extraction & Loss control, timing and beam manipulations \\
\hline Beam Quality & MI/Recycler requirements \\
\hline Operations & Shielding, Booster Hardware \\
\hline \hline
\end{tabular}

\subsubsection{Booster Injection}

\subsubsection{Present Booster Injection}

The Booster lattice contains 24 periods and can be described as a FDooDFo lattice utilizing combined function dipoles with long straight sections $(\sim 5.7 \mathrm{~m})$ between the defocusing (D) dipoles and a short straight section $(\sim 0.9 \mathrm{~m})$ between the focusing (F) dipoles. Horizontal beta-function varies from about 6 meters in the long straight to $33 \mathrm{~m}$ in the short straights while the vertical beta function varies from $20 \mathrm{~m}$ in the long straights to $\sim 5.3 \mathrm{~m}$ in the short straights. The horizontal dispersion varies between approximately 1.2 (in the long straights) and 2.2 meters (in the short straights). Optical functions for one period are shown in Figure 2.33. It should be noted that the optical functions for all periods are almost equal. Differences appear due to optics errors and the focusing effect of the extraction dogleg. The latter is quickly reduced with acceleration and has a negligible effect on the beam optics above $1.5 \mathrm{GeV}$ energy.

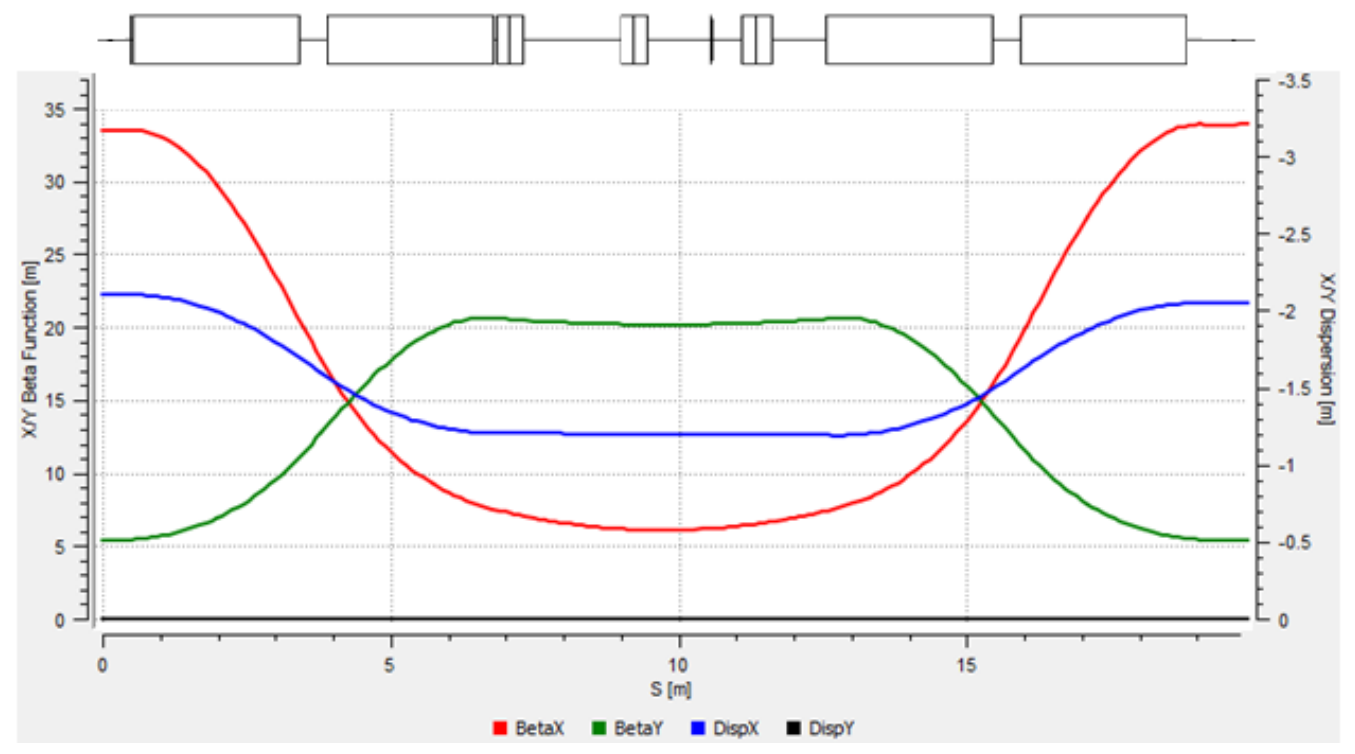

Figure 2.33: Optical functions in the vicinity of existing Booster Long 1 straight section. An arrangement of present injection straight is shown at the top of the plot. The location of pulsed injection dipoles (ORBUMP dipoles) are shown by double rectangles. The vertical line in the center marks position of stripping foil. 
The top part of Figure 2.33 also shows the magnet configuration in the vicinity of the present injection straight section which is considered to be the ring beginning and therefore is named Long 1. The combined function dipoles, pulsed injection dipoles (ORBUMP magnets), and correctors are shown at the top of the figure. The layout of injection area is shown in Figure 2.34. It is based on the three bump system installed in $2006[37,38]$. The center dipole of this insert is used to merge the incoming $\mathrm{H}^{-}$with the proton beam circulating in the ring. The orbit bump is produced by the three pulsed dipoles which displace the closed orbit by $\sim 44 \mathrm{~mm}$ to the stripping carbon foil for the duration of injection ( $\sim 35 \mu \mathrm{s})$. The center dipole runs at twice the field (current) of the outer dipoles. The injection foil is located immediately after the middle ORBUMP magnet. The field in the ORBUMP dipoles does not change during the injection, and there is no phase space painting in any degree of freedom. All three dipoles are powered by a single resonant power supply. The angle produced by the center dipole is approximately $44 \mathrm{mrad}$ which corresponds to an integrated field of $1.4 \mathrm{kG}-\mathrm{m}$. The separation of the ORBUMP magnets is approximately $1.75 \mathrm{~m}$. Lorentz stripping in these magnets is not an issue at $400 \mathrm{MeV}$.

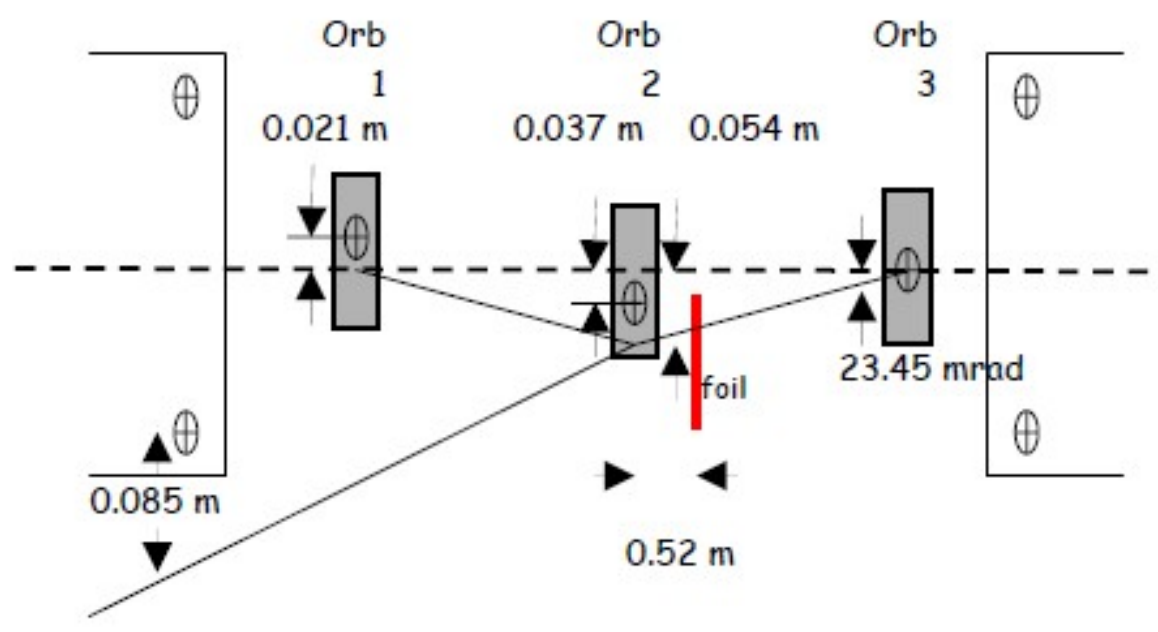

Figure 2.34: The current $400 \mathrm{MeV}$ Horizontal injection insert showing the layout of the three chicane dipoles, foil and injection beam line.

The existing ORBUMP magnets cannot be utilized for $800 \mathrm{MeV}$ injection. Their use would require a magnetic field in the center dipole of $\sim 4.5 \mathrm{kG}$, resulting in unacceptably large Lorentz stripping. There is also insufficient space for the beam dump. Addressing these problems requires a complete redesign of the injection straight.

\subsubsection{Conceptual Design of Booster Injection at $800 \mathrm{MeV}$}

The injection into the Booster during the PIP-II era will be moved from "Long 1" to "Long 11" straight section to facilitate injection from the SC Linac located in the Tevatron infield (Figure 2.35). As already noted, all long straight sections have the same geometry and optics. The flangeto-flange length available for the injection insert is $5.6804 \mathrm{~m}$.

The beam current of the SC Linac $(2 \mathrm{~mA})$ is more than an order of magnitude smaller than that of the present $400 \mathrm{MeV}$ linac. It will require much longer injection time ( 300 turns over $\sim 500 \mathrm{us})$ which together with much smaller linac emittance $\left(\varepsilon_{\mathrm{n}-95 \%} \approx 1.5 \mathrm{~mm}-\mathrm{mrad}\right.$ versus $16 \mathrm{~mm}-\mathrm{mrad}$ specified for the Booster beam) allows us to perform transverse phase space painting. It is expected to be a very efficient cure for suppression of the harmful effects of beam space charge and 
improvement of the longitudinal beam stability. During the phase space painting process, the Booster closed orbit in each plane will be moved by $\sim 2 \sigma$ of the final Booster accumulated phase space (see details below). The vertical displacement will be performed by pulsed magnets of the injection 3-bump, the horizontal displacement by regular Booster dipole correctors.

The conceptual design for the straight section is patterned after the present $400 \mathrm{MeV}$ injection 3bump design by adding a second dipole to the middle bend center. The layout of the injection concept, beam envelopes, and apertures are shown in Figure 2.36. The vertical dimensions of the chicane dipoles and foil changer are represented by the blue boxes and are not to scale. The horizontal dimensions of these devices are roughly to scale. The physical aperture is represented by the black line and the absorber is labeled in the open box. The aperture in the D dipoles is set by the pole tip separation on the central orbit of $2.25 "(+/-28.6 \mathrm{~mm})$. The aperture in the central chicane magnets, PM-2a and PM-2b, is determined by the injected beam trajectory.

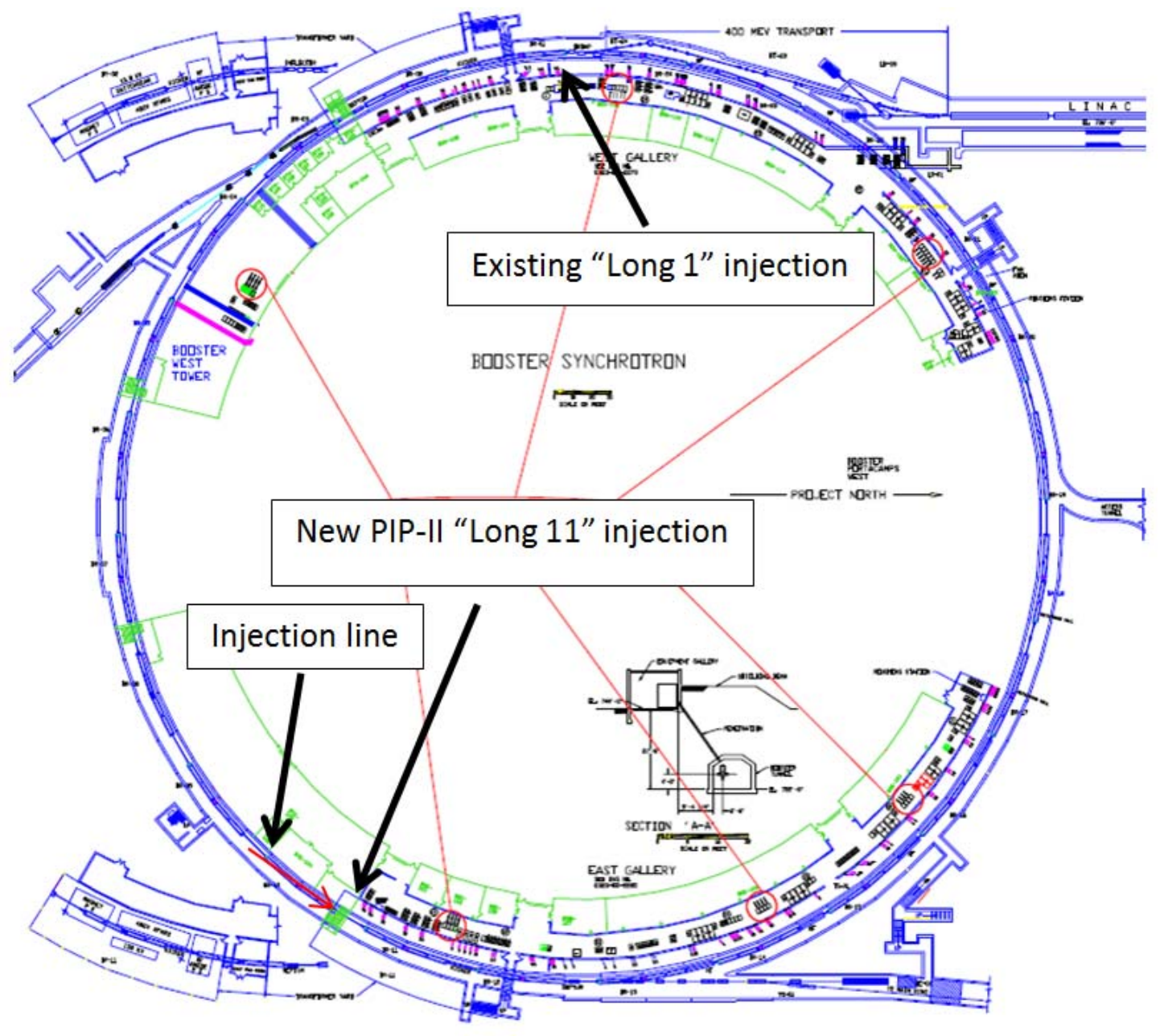

Figure 2.35: Plan view of Booster showing the location of existing and new PIP-II injection insert.

The chicane dipoles move the Booster closed orbit to $45 \mathrm{~mm}$ at the foil location. During the injection process this closed orbit is collapsed by $\sim 2 \sigma$ of the final vertical rms beam size or $\sim 17$ $\mathrm{mm}$ down to $\sim 28 \mathrm{~mm}$. After the end of injection the chicane dipoles return the closed orbit back to zero. The closed orbit displacement in the center of the two central dipoles is approximately 68 $\mathrm{mm}$. The $\mathrm{H}^{-}$injected beam at the foil position is $45 \mathrm{~mm}$ with a vertical angle of $-40 \mathrm{mrad}$. 
Incompletely stripped $\mathrm{H}^{-}$ions (mostly excited states of $\mathrm{H}^{0}$ ) and $\mathrm{H}^{-}$ions which miss the foil will be intercepted by the beam absorber. It is located downstream of the last chicane dipole and upstream of the Booster combined function dipole. For $800 \mathrm{MeV}$ the required space for the dump is $0.5 \mathrm{~m}$ at minimum. There are also other equipment (i.e. correction element package, $0.6 \mathrm{~m}$; vacuum bypass and valve, $0.45 \mathrm{~m}$; ion pumps, $0.2 \mathrm{~m}$; horizontal painting dipole, $\sim 0.2 \mathrm{~m}$, and diagnostics/instrumentation, $\sim 0.25 \mathrm{~m}$ ) which are located in the space between the dipoles.

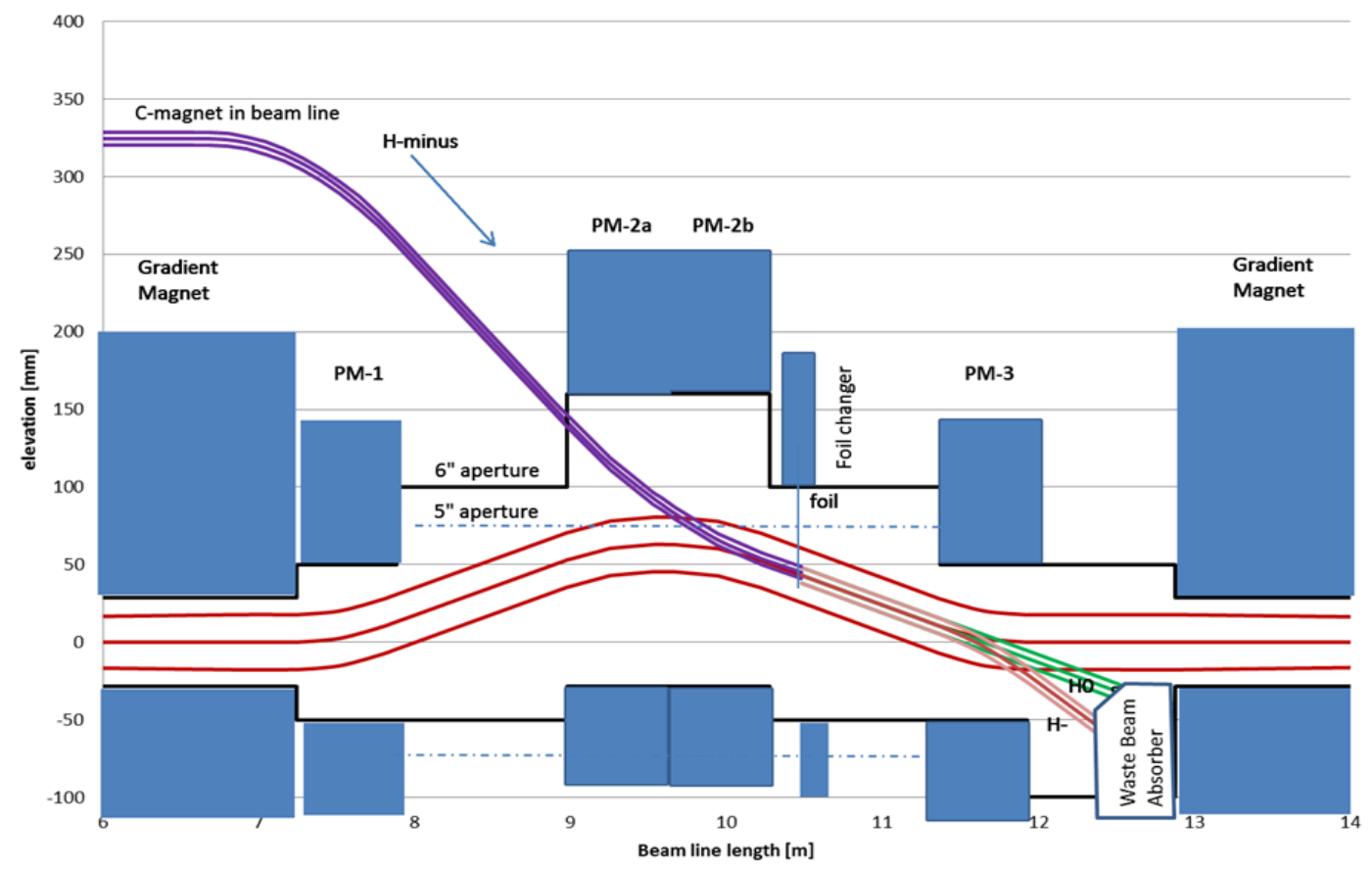

Figure 2.36: Conceptual design for $800 \mathrm{MeV}$ injection using a three-bump chicane within the unmodified length of the long straight section.

Table 2.15: Parameters of elements located in the injection straight

\begin{tabular}{l|l|c|c|c|c}
\hline \hline$\#$ & \multicolumn{1}{|c|}{ Name } & $\begin{array}{c}\text { Accumulated } \\
\text { Length }(\mathrm{m})\end{array}$ & $\begin{array}{c}\text { Flange-Flange or Drift } \\
\text { Length }(\mathrm{m})\end{array}$ & $\begin{array}{c}\text { Magnetic } \\
\text { Length }(\mathrm{m})\end{array}$ & $\begin{array}{c}\text { Magnetic } \\
\text { Field (kG) }\end{array}$ \\
\hline 1 & Drift 1 & .07067 & 0.07067 & & \\
\hline 2 & ORBUMP 1 & 0.7624 & 0.69171 & 0.5585 & 3.496 \\
\hline 3 & Drift 2 & 1.7724 & 1.01 & & \\
\hline 4 & ORBUMP2a & 2.4641 & 0.69171 & 0.5585 & 3.496 \\
\hline 5 & ORBUMP2b & 3.1558 & 0.69171 & 0.5585 & 3.496 \\
\hline 6 & Drift 3 & 3.2570 & 0.1012 & & \\
\hline 7 & Foil changer & 3.5617 & 0.3047 & & \\
\hline 8 & Drift 4 & 4.1667 & 0.6050 & & 3.496 \\
\hline 9 & ORBUMP 3 & 4.8584 & 0.69171 & 0.5585 & \\
\hline 10 & Drift 5 & 5.1584 & 0.3 & & \\
\hline 11 & Absorber & 5.6584 & .05 & & \\
\hline 12 & Drift & 5.6804 & 0.02202 & & \\
\hline \hline
\end{tabular}


The current placement of the C-dipole in the transport beam line is just over the upstream Booster dipole with beam elevation of $\sim 0.15 \mathrm{~m}$ above the top of steel of the Booster dipole. The bend angle of the $\mathrm{C}$-dipole is 3 times the angle of the chicane dipoles or approximately $120 \mathrm{mrad}$. The length of this magnet will be chosen to keep the peak dipole field at a level to minimize Lorentz stripping of the incoming $\mathrm{H}^{-}$ions, i.e. less than $4 \mathrm{kG}$. Table 2.15 present lengths allocated for different elements and their parameters. The accumulated length is between flanges of the gradient magnets at either end of the straight section and is given at the end of each element. In addition to the elements in the Booster ring, the last magnet in the transfer line (C-dipole) ends 0.505 meters upstream of the ORBUMP2 magnet. It has a magnetic length of 1.8 meters with an angle of $-120 \mathrm{mrad}$ and field of $3.26 \mathrm{kG}$.

\subsubsection{Phase Space Painting}

Small values of beam emittances of the linac beam allow us to perform phase space painting in all degrees of freedom. It reduces harmful effect of the beam space charge on the transverse particle motion due to reduction of space charge incoherent tune shifts and is expected to be helpful in achieving longitudinal stability (see below).
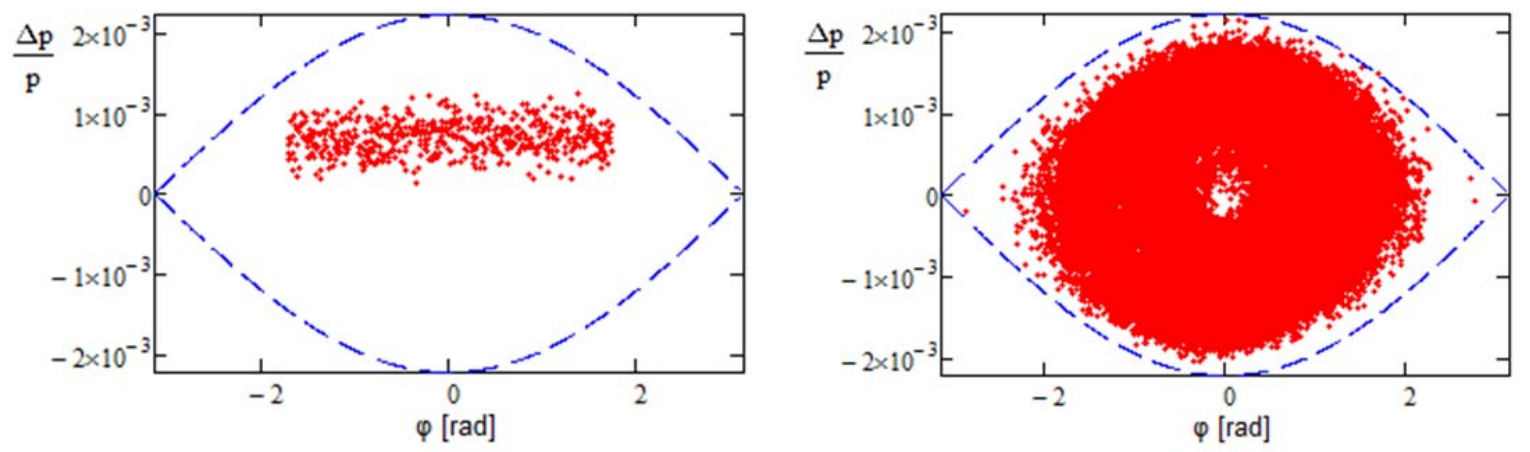

Figure 2.37: The longitudinal phase space of particles incoming to the Booster (left) and the phase space at the end of injection process (right).

The rms momentum spread of linac beam of $2 \cdot 10^{-4}$ is an order of magnitude smaller than the RF bucket height in the Booster $\left(2.2 \cdot 10^{-3}\right)$. Together with the long duration of the injection process corresponding to 7 synchrotron periods it enables static longitudinal painting, which greatly simplifies the procedure. In this case the linac energy is offset relative to the Booster reference energy and the synchrotron motion mixes particles in the longitudinal phase space in the course of injection. Linac bunches that would be outside of RF buckets are removed by the bunch-by-bunch chopper in the MEBT. Figure 2.37 presents the longitudinal phase spaces of injected beam and the Booster beam at the injection end. The value of the momentum offset $\left(7 \cdot 10^{-4}\right)$ and the duration of the injection window (55\%) were optimized to minimize the bunching factor. Figure 2.38 presents the longitudinal density along a Booster bunch. The corresponding bunching factor is 2.5. In average each Booster RF bucket receives two linac bunches per injection turn. The linac bunch frequency of $162.5 \mathrm{MHz}$ and the Booster RF frequency of $44.705 \mathrm{MHz}$ are not related as integers and therefore the injection process is asynchronous. It results in a variation in number of injected linac bunches in the range of $\pm 0.34 \%$ ( \pm 2 bunches out of 588 bunches injected into an RF bucket per turn). The rms bunch lengthening in the course of beam transport from linac to Booster is about $14 \mathrm{~mm}$. This value is much smaller that the RF bucket length of $5.65 \mathrm{~m}$ and can be neglected in 
most practical considerations.

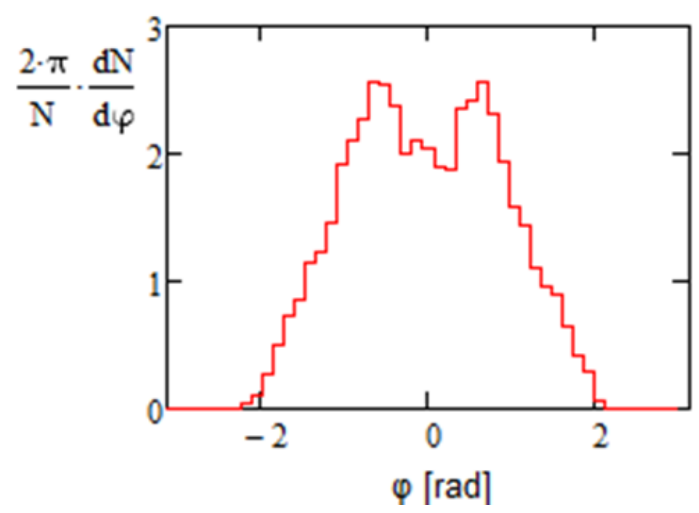

Figure 2.38: The longitudinal density of an injected bunch after injection.

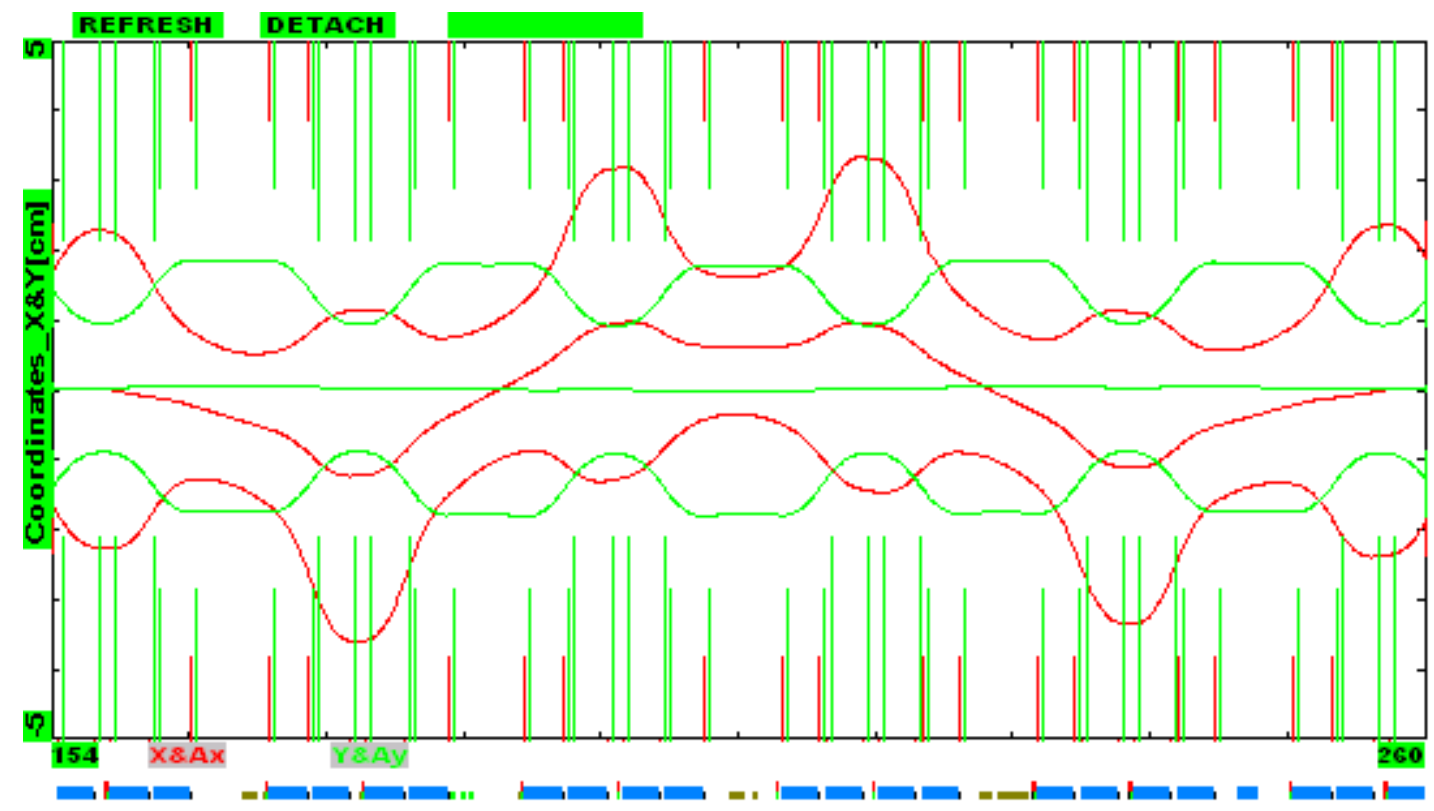

Figure 2.39: Horizontal orbit bump for beam painting at the maximum horizontal (red) and zero vertical (green) displacements. Beam envelopes for the normalized acceptance of $25 \mathrm{~mm}$ mrad are also shown. The injection straight (Long 11) is in the center of the plot $(s \in[204.3-210.3] \mathrm{m})$. Vertical lines show aperture limitations in the dipoles (horizontal - red, vertical green).

As mentioned above, the phase space painting in the vertical plane is performed by the dipoles of the injection chicane. Phase space painting in the horizontal plane will be performed by regular Booster correctors located outside of the injection straight. The correctors should create a closed orbit bump with maximum beam displacement on the foil of $6.1 \mathrm{~mm}$. The orbit bump implemented with only the correctors closest to the injection straight (HL10, HS10, HS11, HL12) has minimal extent outside of the injection straight. However, it requires considerable corrector strength of 6.3 $\mathrm{kG} \cdot \mathrm{cm}$. Although the strength of the present horizontal correctors of $9 \mathrm{kG} \cdot \mathrm{cm}$ is sufficient, their slew rate of $3.24 \mathrm{kG} \cdot \mathrm{cm} / \mathrm{ms}$ is about 5 times less than the slew rate required for painting. Therefore a longer bump using correctors from nearby short straights looks preferable. Table 2.16 presents the corrector strengths and Figure 2.39 shows the corresponding beam displacement together with 
the beam envelope in the vicinity of injection straight. The envelope is plotted for the normalized emittance of $25 \mathrm{~mm} \cdot \mathrm{mrad}$ which includes close to $100 \%$ of the particles (see below). The horizontal beta-function in the short straights is about 5 times smaller than in the long ones. It makes the main contribution to a reduction of corrector strengths by $\sim 3.4$ times. The required slew rate of $4.5 \mathrm{kG} \cdot \mathrm{cm} / \mathrm{ms}$ still exceeds the slew rate for present correctors by about 1.4 times. This problem can be addressed by an upgrade of corrector power supplies or by usage of a longer orbit bump.

Table 2.16: Corrector strengths required to create horizontal injection orbit bump

$\begin{array}{lccccc}\text { Name } & \text { HS08 } & \text { HS09 } & \text { HS011 } & \text { HS12 } & \text { HS13 } \\ \text { BdL }[\mathrm{kG} \cdot \mathrm{cm}] & -1.867 & 0.72 & 0.192 & 0.72 & -1.723\end{array}$
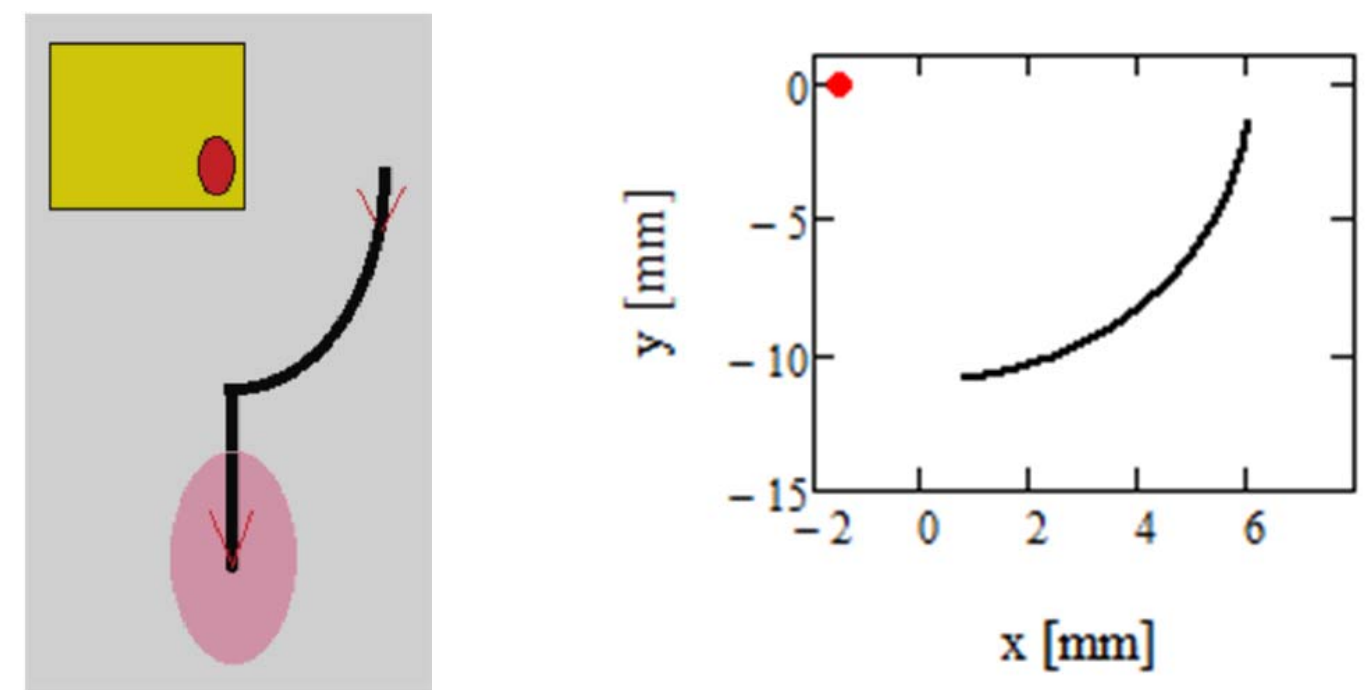

Figure 2.40: Schematic of injection painting (left pane): square (yellow) - stripping foil, small ellipse (red) - linac beam hitting the foil, large ellipse (pink) - circulating beam after the end of injection process, black line - trajectory of closed orbit displacements in the course of beam painting. Right pane - plot of closed orbit displacements in the course of beam painting relative to the linac beam center (shown by the red dot). The reference orbit stays at zero $x$-coordinate. The linac beam is displaced outward by $1.5 \mathrm{~mm}$ to account for the energy offset $\left(\Delta p / p=7 \cdot 10^{-4}\right)$ required for the longitudinal painting. Positive values of beam displacement in the horizontal and vertical planes correspond to inward and upward displacements, respectively (consequence of the righthand coordinate frame chosen for description of ring orbit and optics).

The stripping foil and the injected $\mathrm{H}^{-}$beam do not move during injection (see Figure 2.40). Similar to the beam injection in the SNS the rectangular foil is hanged in the vacuum and the linac beam hits it near the corner to minimize number of secondary foil hits by particles of already injected beam. Both betatron and synchrotron oscillations in average push particles out of the foil.

The horizontal and vertical displacements of the closed orbit are correlated and follow the ellipse as shown in Figure 2.40. The ellipse semi-axes are 6.1 and $11 \mathrm{~mm}$ for horizontal and vertical amplitudes, correspondingly. To reduce number of foil secondary hits and improve the uniformity of the distribution the painting curve covers only $84 \%$ of the ellipse quarter as shown in the right pane of Figure 2.40. That results in the actual beam displacements of 5.3 and $9.5 \mathrm{~mm}$ for 
horizontal and vertical planes, respectively. After painting the closed orbit and, consequently, the beam are moved vertically down to the nominal (central) orbit. As can be seen in Figure 2.40 the center of linac beam is additionally displaced outward by $1.5 \mathrm{~mm}$ to account for the energy offset required for the longitudinal painting. It also reduces the number of foil hits because the synchrotron motion keeps the center of accumulated beam at zero horizontal coordinate.

The limited space available for the beam injection and small vertical aperture of Booster dipoles forces us to make vertical painting by changing magnetic field of the chicane. Unfortunately it also affects the vertical position of linac beam on the foil. This displacement is induced by changing magnetic field of the central chicane dipole (see Figure 2.36) as the linac beam has to come through it. This beam position change is corrected by fast dipole correctors located at the end of transport line.

To minimize the number of passages through the foil the beta- and alpha-functions of the linac beam are scaled from the corresponding values of the Booster by factor of 0.483 so that the linac phase space would be inscribed into the $x$ and $y$ machine acceptances as shown in Figure 2.41. The offsets of linac beam relative to the Booster beam presented in Figure 2.41 are equal to the painting offsets discussed above. Table 2.17 presents Twiss parameters for the linac and Booster beams on the stripping foil. To minimize displacements of linac beam position on the stripping foil we require its dispersions and their derivatives to be equal to zero. The emittance increase related to this dispersion mismatch is negligible. The number of secondary foil hits increases quickly with distance between the linac beam center and the foil edges, i.e. if the foil is moved closer to the circulating beam. To minimize the foil hits we assumed that one percent of the linac beam can miss the foil. For a Gaussian beam it determines that the distances has to be 2.58 times of the corresponding rms beam sizes or $1.95 \mathrm{~mm}$ and $3.51 \mathrm{~mm}$ for the horizontal and vertical planes, respectively.
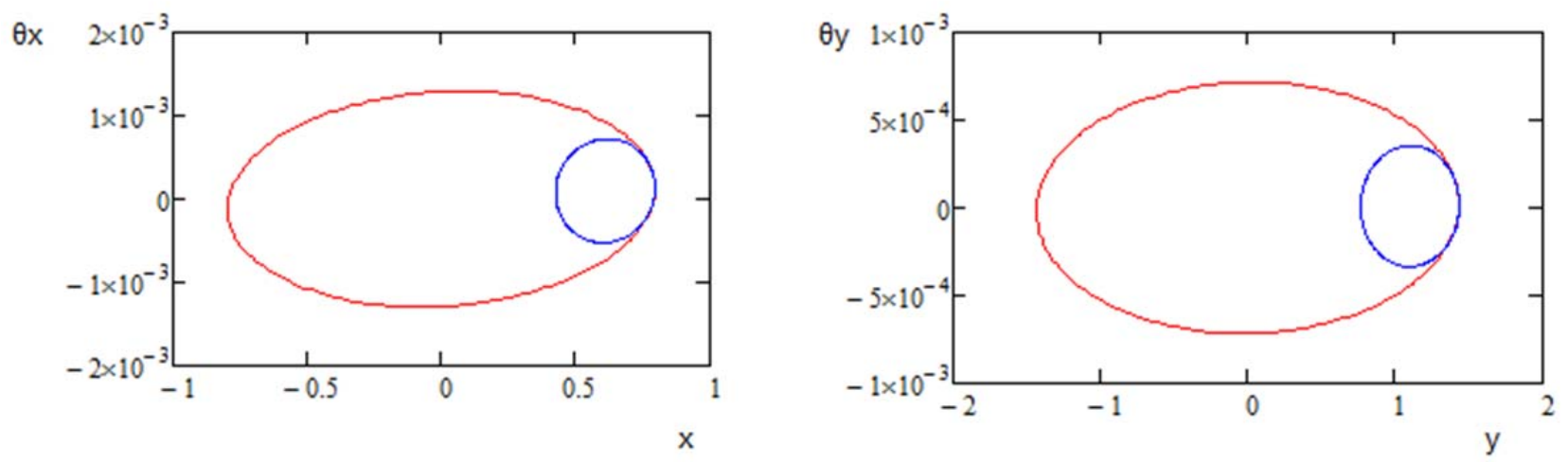

Figure 2.41: Phase space boundaries of the linac (blue lines) and RCS (red lines) beams. The linac beam boundaries correspond to the normalized boundary emittance of $1.8 \mathrm{~mm} \cdot \mathrm{mrad}(95 \%$ normalized linac emittance) the RCS beam boundaries correspond to the normalized emittances of $16 \mathrm{~mm}$ mrad.

Figure 2.42 presents results of experimental measurements of $\mathrm{H}^{-}$stripping by a carbon foil at $800 \mathrm{MeV}$, as presented in Ref. [39], with their extrapolation for a thicker foil. The extrapolation assumes the following cross-sections for transitions: $\sigma_{\mathrm{H}-\rightarrow \mathrm{H} 0}=67.6 \cdot 10^{-16} \mathrm{~cm}^{-2}, \sigma_{\mathrm{H}-\rightarrow \mathrm{H}^{+}}=1.2 \cdot 10^{-16} \mathrm{~cm}^{-2}$ and $\sigma_{\mathrm{H} 0 \rightarrow \mathrm{H}^{+}}=26.4 \cdot 10^{-16} \mathrm{~cm}^{-2}$ [40]. It also assumes that there are no transitions with electron capture i.e. $\mathrm{H}^{0} \rightarrow \mathrm{H}^{-}, \mathrm{H}^{+} \rightarrow \mathrm{H}^{-}$and $\mathrm{H}^{+} \rightarrow \mathrm{H}^{0}$. This assumption is well justified for $800 \mathrm{MeV}$ energy. The thickness of stripping foil was chosen to be $600 \mu \mathrm{g} / \mathrm{cm}^{2}$. This is thick enough to strip the major 
fraction of $\mathrm{H}^{-}$to protons leaving less than $0.1 \%$ particles as $\mathrm{H}^{0}$ and a negligible fraction as $\mathrm{H}^{-}$. As shown below, this thickness does not cause problems with particle scattering in the foil and foil overheating but should support longer foil operation than a thinner foil. Spattering resulting in foil evaporation is expected to be a major mechanism limiting the foil lifetime. As one can see from Figure 2.42, operation with foil thickness as thin as $400 \mu \mathrm{g} / \mathrm{cm}^{2}$ is still possible.

Table 2.17: Twiss parameters for the Booster and linac beams at the stripping foil

\begin{tabular}{l|c|c|c|c|c|c}
\hline \hline & $\beta_{x}(\mathrm{~m})$ & $\alpha_{x}$ & $\beta_{y}(\mathrm{~m})$ & $\alpha_{y}$ & $D_{x}(\mathrm{~m})$ & $D^{\prime}{ }_{x}$ \\
\hline Booster & 6.17 & -0.095 & 20.03 & -0.028 & 2.18 & 0 \\
\hline Linac & 2.98 & -0.046 & 9.67 & -0.014 & 0 & 0 \\
\hline \hline
\end{tabular}
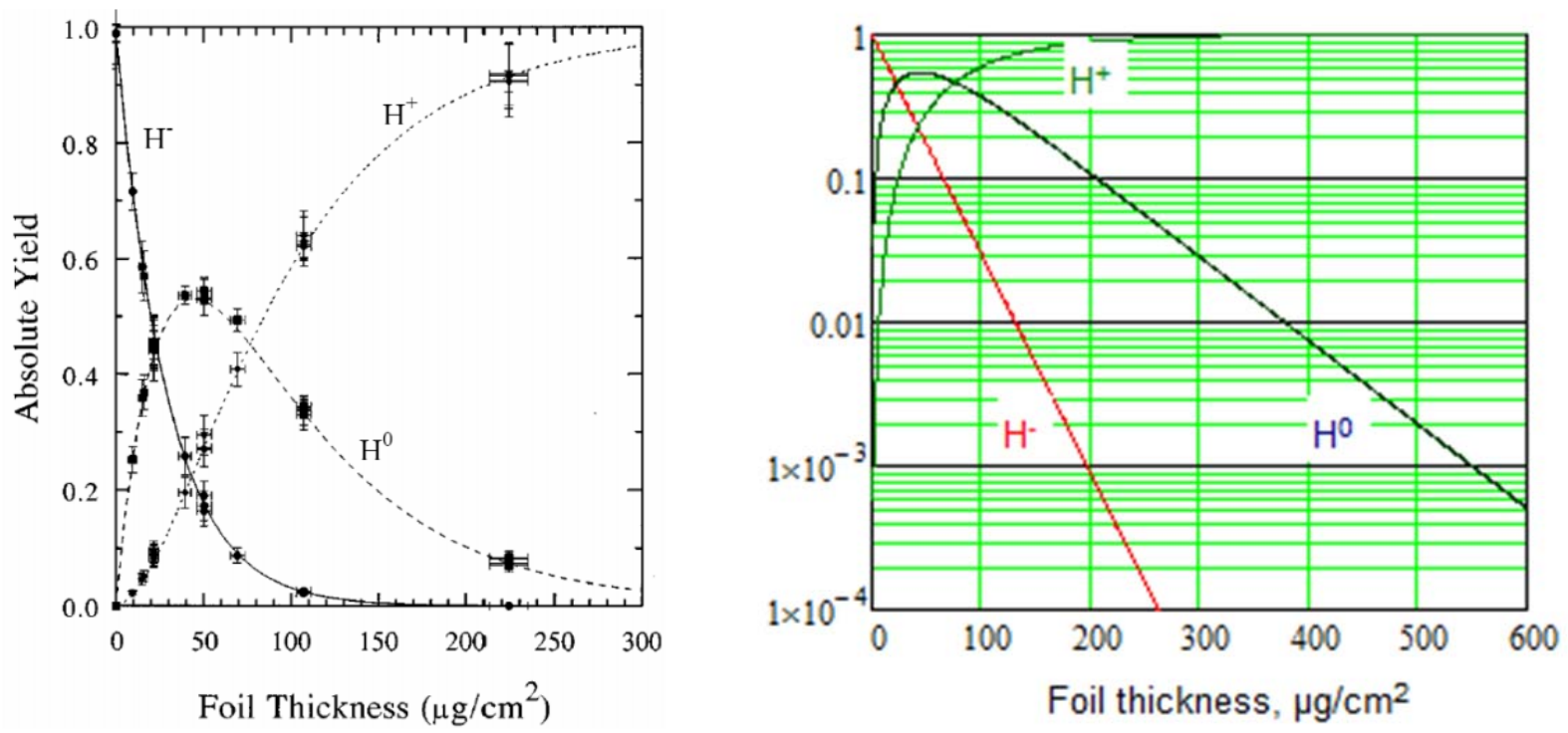

Figure 2.42: Measurement of $\mathrm{H}^{-}$stripping by carbon foil at $800 \mathrm{MeV}$ presented in Ref. [39] (left pane) and their extrapolation for a thicker foil (right pane).
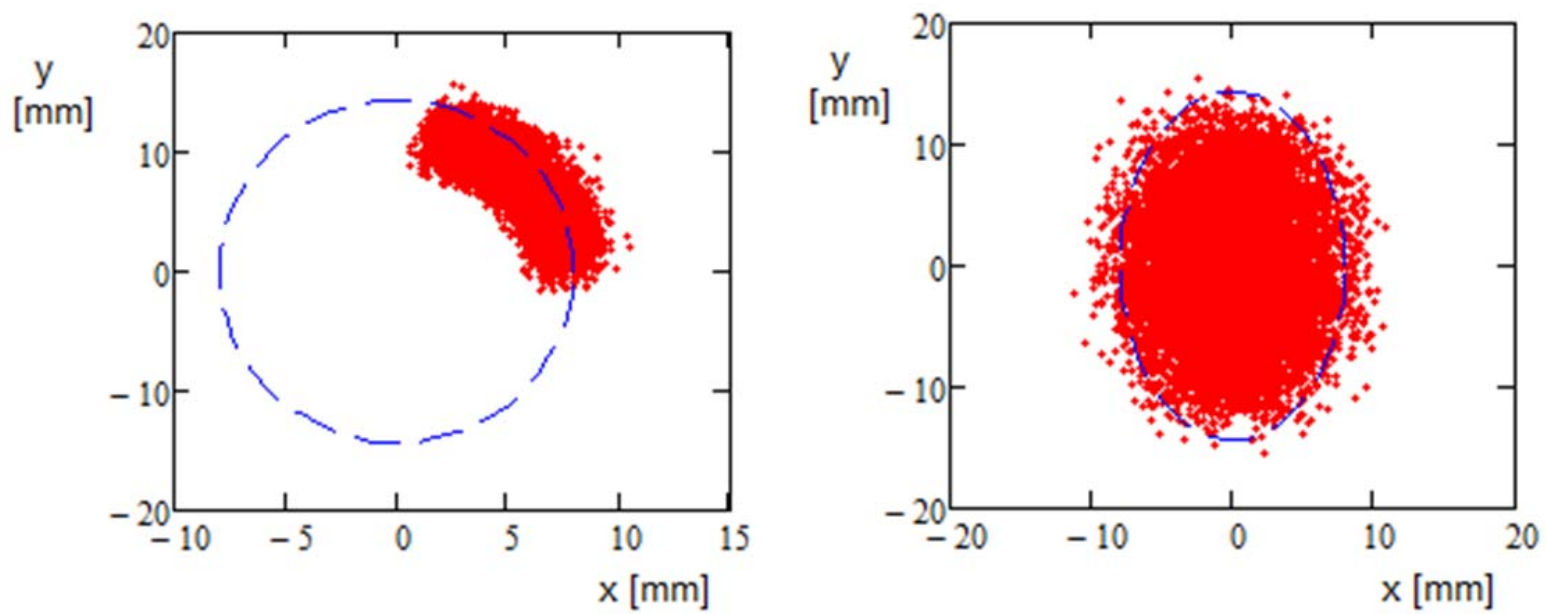

Figure 2.43: $\mathrm{X}$ and $\mathrm{Y}$ coordinates of all injected (in simulations) particles relative to the current orbit position for particles incoming to the Booster (left) and at the end of injection process (right). 
Figure 2.43-2.46 present results of numerical simulation of the strip injection. The simulation includes multiple scattering in the foil, synchrotron and betatron motions and the details of the painting process described above. The beam space charge effects are not taken into account and betatron motion is linear and without $x-y$ coupling. The simulation showed that the betatron tunes have to be different by more than 0.01 for uniform painting. The results were obtained for the following tunes: $Q_{x}=6.8$ and $Q_{y}=6.81$. Tune values have little effect on the result as long as they are different. The left pane in Figure 2.43 presents $x$ and $y$ coordinates of all particles which were injected at their arrival on the foil. The coordinates are referenced to the reference orbit position at the arrival time. The right pane presents particle coordinates at the end of the injection process. The left pane in Figure 2.44 shows the particle distribution in Courant-Snyder invariants (single particle emittances). One can see that the distribution is somewhere between the Gaussian and $\mathrm{K}-\mathrm{V}$ distributions. The latter would be represented by the $\delta$-function, $\delta\left(\varepsilon_{4 D n}-\varepsilon_{b n}\right)$, in the 4dimensional space, where $\varepsilon_{4 D n}=\varepsilon_{x n}+\varepsilon_{y n}$, and $\varepsilon_{b}$ is the normalized boundary emittance of KVdistribution. The right pane in Figure 2.44 shows the integrals of the particle distributions presented in the left pane normalized to 1 . One can see that $95 \%$ of particles are within $17 \mathrm{~mm} \mathrm{mrad}$ and almost $100 \%$ within $23 \mathrm{~mm}$ mrad. Similarly, Figure 2.45 presents the longitudinal distribution and its integral. As one can see $100 \%$ of particles are within $0.06 \mathrm{eV} \cdot \mathrm{s}$.
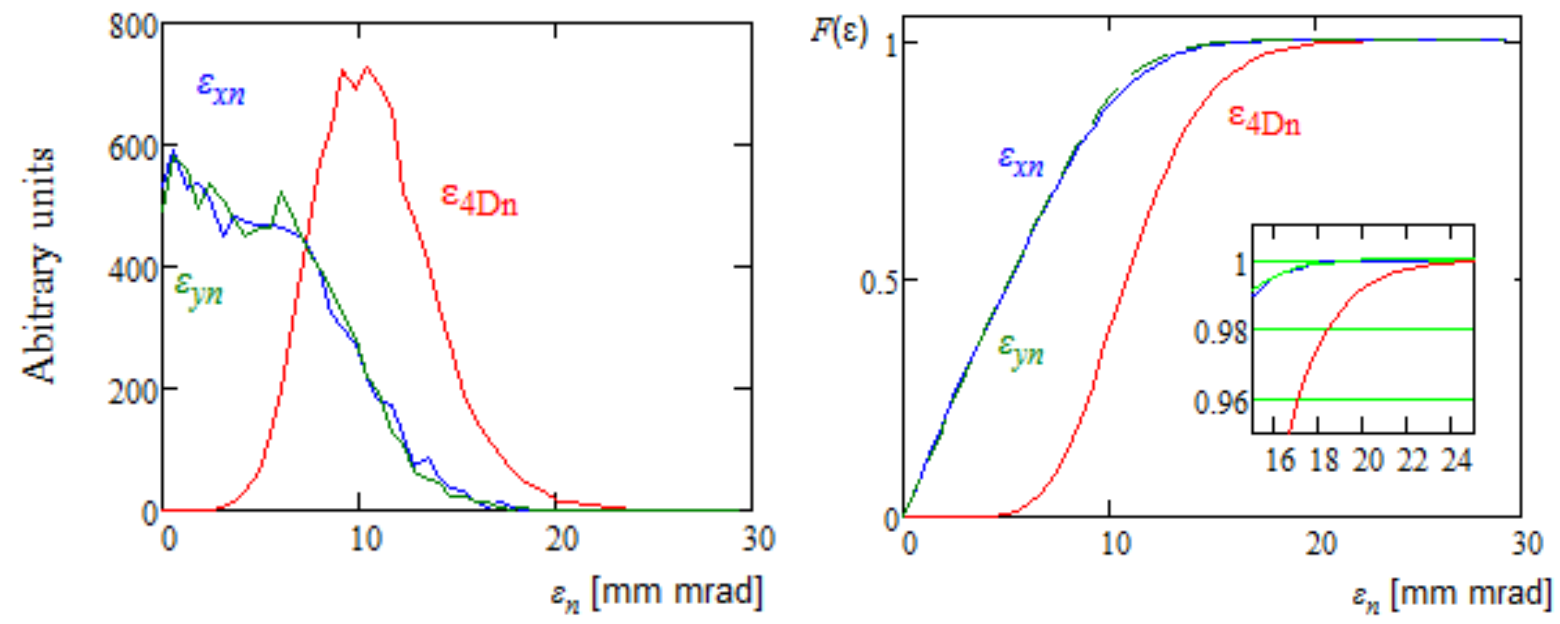

Figure 2.44: Left pane - the particle distribution over particle normalized Courant-Snyder invariant: $\varepsilon_{x n}=\beta \gamma\left(x^{2} / \beta_{x}+2 \alpha_{x} x \theta_{x}+\left(1+\alpha_{x}^{2}\right) \theta_{x}^{2} / \beta_{x}\right)$, (and similar for $y$ plane); blue - horizontal plane, green - vertical plane, red - the distribution over sum of invariants, $\varepsilon_{4 D}=\varepsilon_{x}+\varepsilon_{y}$. Right pane - the integrals of particle distributions (normalized to unity) presented in the left pane. The insert shows detail near the top of the picture.

Figure 2.46 presents the distribution of secondary and primary hits of the surface of stripping foil. The peak of secondary hits is located at the foil corner and is equal to 63 hits per particle per $\mathrm{cm}^{2}$. The average number of secondary foil hits is 6.1 per injected particle. The distribution of primary hits is peaked at the center of incoming linac beam and is about 4 times smaller (15.4 hits per particle per $\mathrm{cm}^{2}$ ). As can be seen from the right pane in Figure 2.46 the peak of the total (summed for primary and secondary hits) hit distribution is determined by secondary hits. 

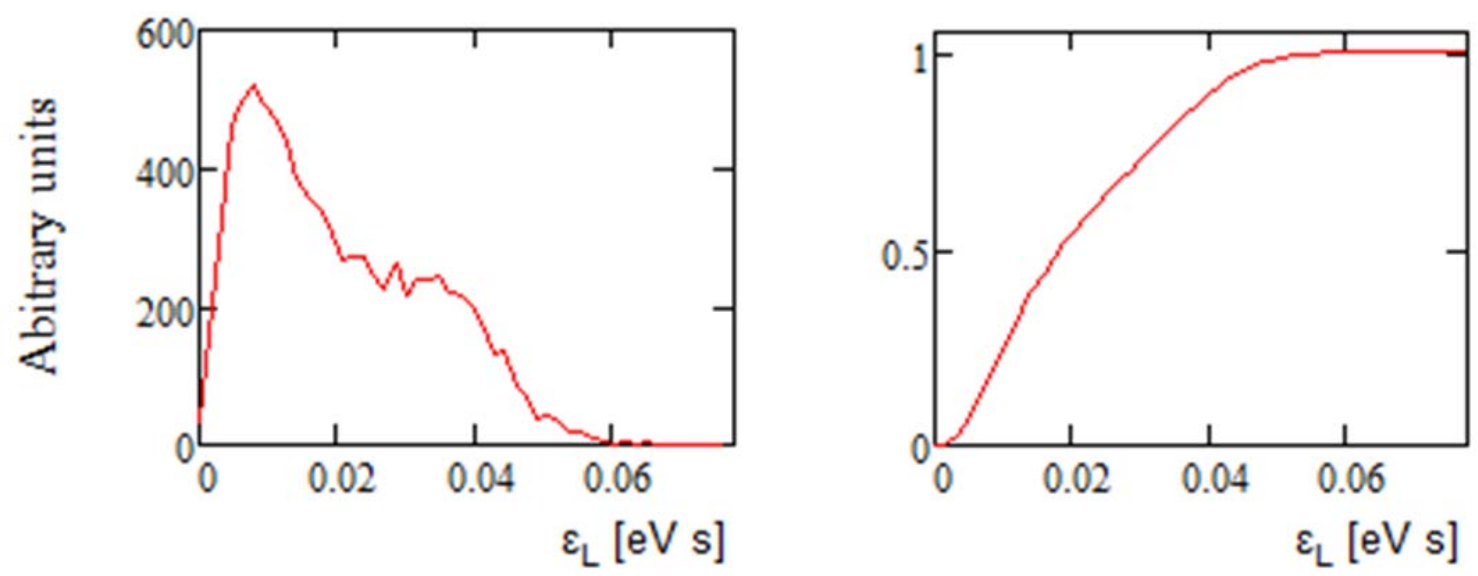

Figure 2.45: Left pane - the particle distribution over particle longitudinal emittance (phase space area subtended by particle trajectory). Right pane - the normalized to one integral of particle distribution presented in the left pane. The horizontal axis ends at the bucket boundary.

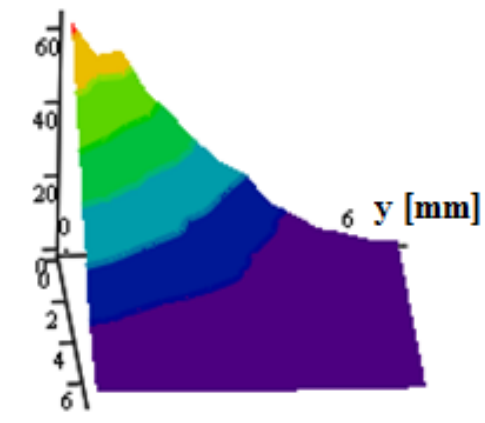

$\mathbf{x}[\mathrm{mm}]$

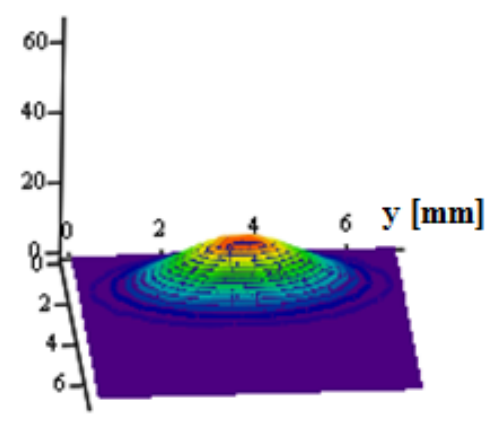

$\mathbf{x}[\mathrm{mm}]$

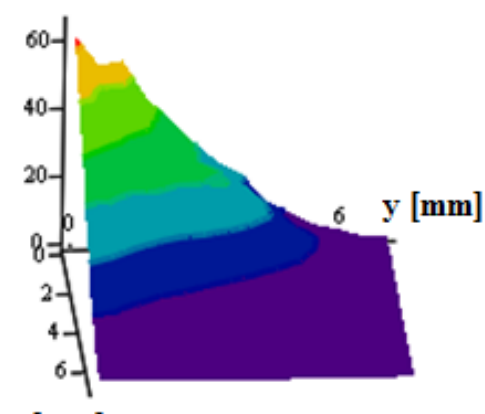

$\mathbf{x}[\mathrm{mm}]$

Figure 2.46: The distribution of particle hits on the stripping foil (hits per injected particle per $\mathrm{cm}^{2}$ ): left - secondary hits, center - primary hits if incoming linac beam, right - sum of primary and secondary hits.

The beam passing through the foil results in its heating. Although the total deposited power of $33 \mathrm{~mW}$ is small, the power density is still considerable due to small size of the injected beam. At its peak in the foil corner the power density is about $2 \mathrm{~W} / \mathrm{cm}^{2}$. The major cooling mechanism for the foil is the black body radiation. Conservatively assuming the foil emissivity of $50 \%$ one obtains the peak temperature of $640 \mathrm{C}^{\circ}$ at the foil corner. This temperature is sufficiently small to guarantee a long lifetime for the foil. Figure 2.47 presents a dependence of the hottest spot temperature on time after initiation of beam operation. The temperature reaches its peak after the fourth pulse. An estimate shows that accounting for the foil thermal conductivity yields a quite small correction and it was neglected in the above calculation.

The total power of the injected beam is about $18 \mathrm{~kW}$. About $1.2 \%$ of these particles are expected to be lost during injection: $\sim 1 \%$ miss the foil, $0.1 \%$ are not completely stripped in the foil, and $0.1 \%$ are lost due to single scattering in the foil. In normal operating conditions the resulting heat load on the injection beam dump is about $200 \mathrm{~W}$ with $20 \mathrm{~W}$ of uncontrolled beam loss mostly intercepted in the first two dipoles.

Stripping of $\mathrm{H}^{-}$also yields two $400 \mathrm{keV}$ electrons for each stripped $\mathrm{H}^{-}$. These electrons carry a 
power of about $18 \mathrm{~W}$ that needs to be intercepted by the electron beam dump. After leaving the foil the electrons are reflected from the downstream dipole where they are bent by its magnetic field. It results in their bending by $180 \mathrm{deg}$. and displacement by a few centimeters in the vertical plane. The design of the electron dump must prevent the interaction of secondary electrons with the circulating beam.

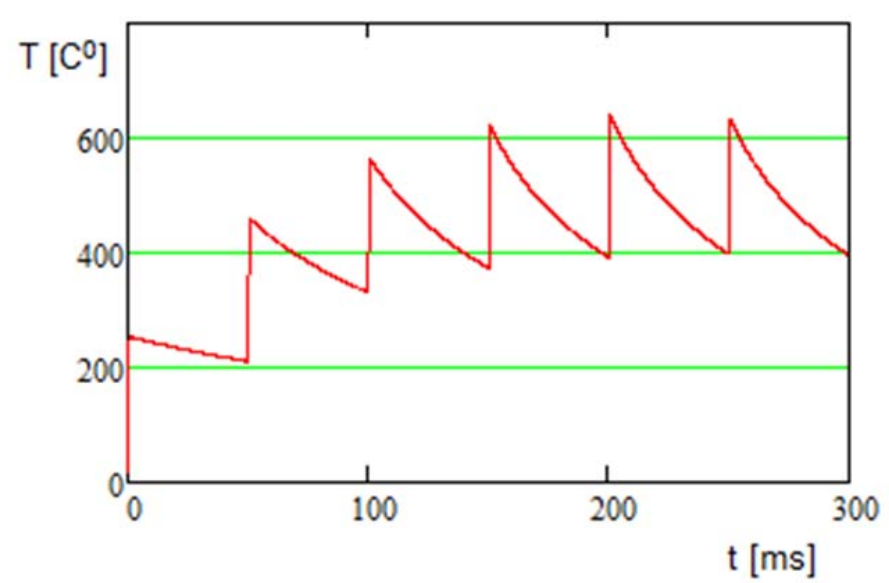

Figure 2.47: Dependence of temperature on time for hottest place on the foil; $t=0$ corresponds to the first injection pulse.

The injection to the Recycler, and, subsequently, to MI requires injection gaps of 3 buckets in the bunch structure of Booster beam. Removal of these bunches will be performed by the bunchby-bunch chopper located in the linac MEBT.

\subsubsection{Beam Acceleration in the Booster}

The longitudinal emittance of the Booster beam is limited by the RF bucket size in the Recycler. Although the RF bucket size in the Recycler will be larger by 33\% due to an increase of Booster repetition rate from 15 to $20 \mathrm{~Hz}$ (see details below) the longitudinal rms emittance of the Booster beam is expected to be the same. A larger ratio of bucket size to the beam emittance should enable a reduction of beam loss by factor more than 2 , tentatively from $5 \%$ to $2 \%$. Thus, the RF bucket size in the Booster is expected to be the same as for PIP. An increase of the Booster ramp rate increases the magnetic field ramping rate and the RF voltage required for acceleration. However, an increase of the RF voltage is not necessarily required if the RF bucket area is the same. Actually, to keep a desirable size of RF bucket during acceleration in the present Booster the maximum RF voltage is required at the cycle beginning ( $\sim 5 \mathrm{~ms}$ after injection) when the accelerating rate is still comparatively small. The decrease of the slip factor with higher injection energy of the PIP-II reduces RF voltage required for the longitudinal beam focusing, thus resulting in about the same requirements for the peak RF voltage. Figure 2.48 presents time dependences of beam and RF system parameters in the course of acceleration. The dependencies were computed assuming small beam current and adiabatic longitudinal motion in the course of acceleration. The latter is not true in the close vicinity of transition and therefore the divergences for the bucket area and the bucket height do not describe actual beam behavior. The plots of the bottom row were computed by turn-by-turn particle tracking and therefore they describe the transition crossing accurately if the beam intensity is sufficiently small. Note that the dependence of RF voltage on time does not include voltage manipulations necessary for successful transition crossing with high intensity beam. As will be shown below these manipulations require up to $1 \mathrm{MV} /$ turn RF voltage. 
Reliable operation with such voltage requires $22 \mathrm{RF}$ cavities (stations). This is 3 more than expected to be present at the onset of PIP operation. The present RF cavities and power amplifiers are adequate for the beam acceleration although minor modifications to the power amplifiers will be required due to 1.5 times larger beam power. Detailed simulations of transition crossing are presented in the two following subsections.

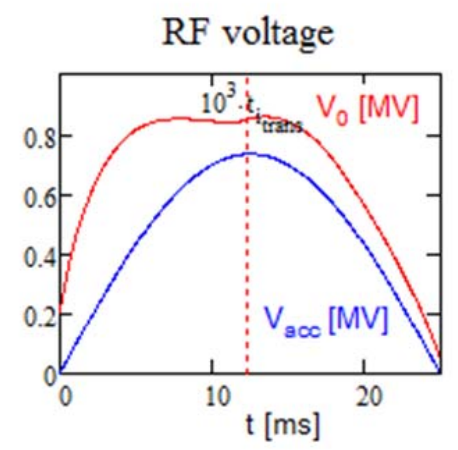

Bucket height, $\Delta \mathrm{P} / \mathrm{P}$
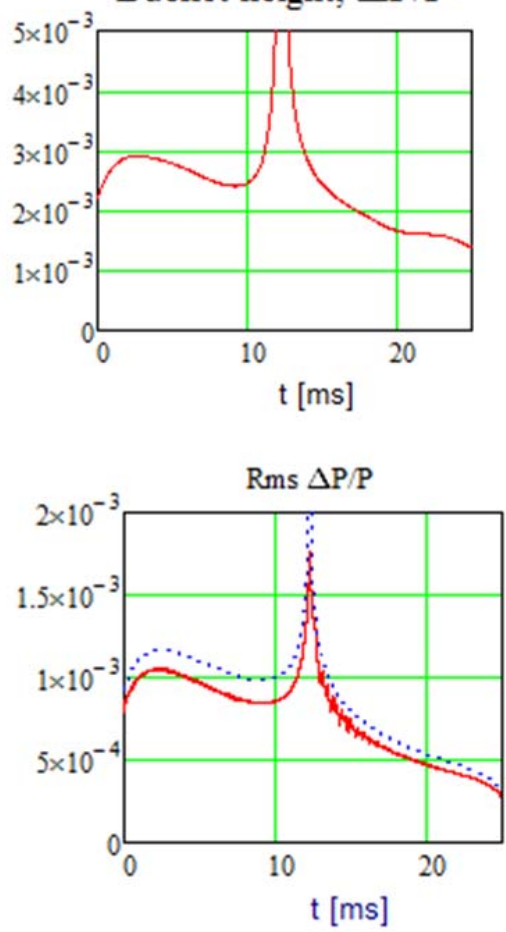

Accelerating phase, deg

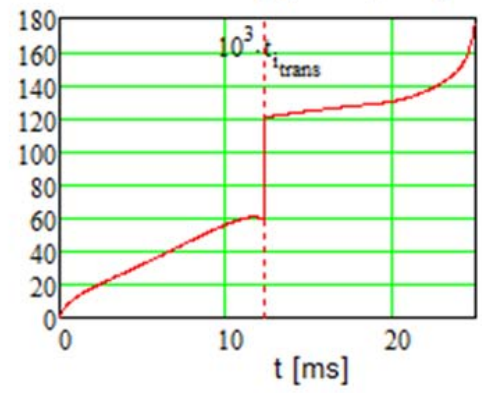

Bucket length, deg

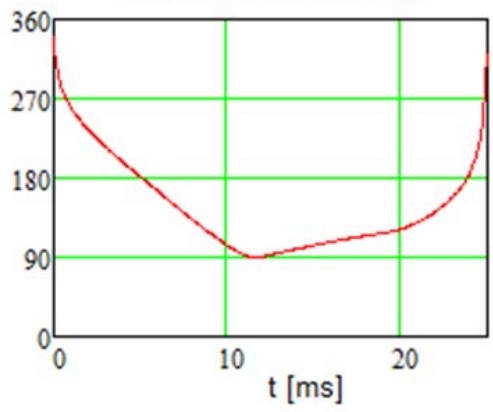

Rms bunch length, deg

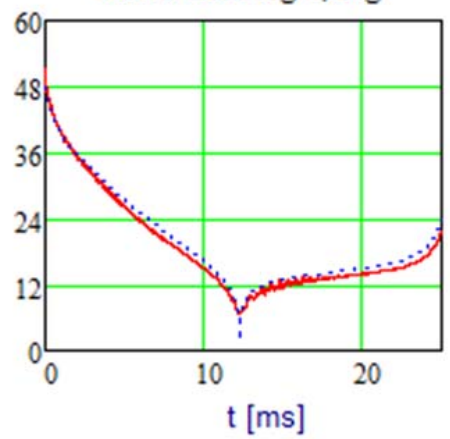

Bucket area, eV s

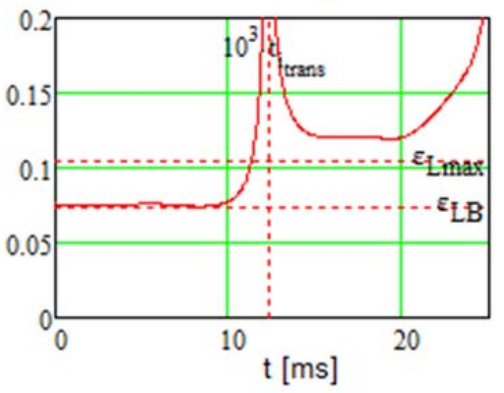

Synchrotron tune

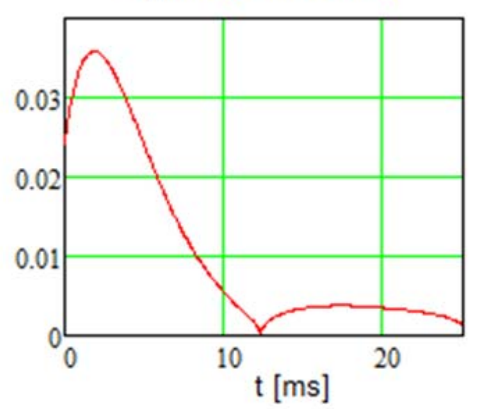

Bunching factor

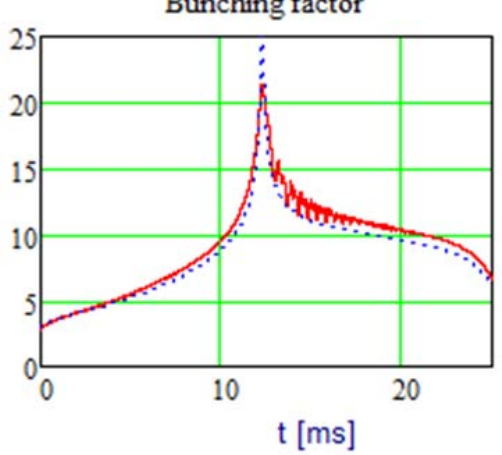

Figure 2.48: Beam and RF system parameters during acceleration. The initial RF bucket size is $0.075 \mathrm{eV} \cdot \mathrm{s}$, and the $100 \%$ initial longitudinal emittance is equal to $0.06 \mathrm{eV} \cdot \mathrm{s}$. The red lines in the bottom row present the tracking results for a small intensity bunch, and the blue lines the results of adiabatic approximation.

Compared to the present Booster operation, the injection energy increase combined with the beam painting result in a significant decrease of the incoherent tune spread due to beam space charge. This effect is usually characterized by the space charge tune shift for particles with small betatron and synchrotron amplitudes, which, for a Gaussian bunch, is equal to:

$$
\delta v_{S C_{x, y}}=-\frac{r_{p} N_{b} B}{2 \pi \beta^{2} \gamma^{3}}\left\langle\frac{\beta_{x, y}}{\sigma_{x, y}\left(\sigma_{x}+\sigma_{x}\right)}\right\rangle \frac{C}{L_{b}} .
$$


Here $N_{b}$ is the number of particles per bunch, $\beta$ and $\gamma$ are relativistic factors, $\sigma_{x}=\sqrt{\varepsilon_{x} \beta_{x}+D_{x}^{2} \sigma_{p}^{2}}$ and $\sigma_{y}=\sqrt{\varepsilon_{y} \beta_{y}}$ are the rms beam sizes, $\beta_{x}$ and $\beta_{y}$ are the beta-functions, $D_{x}$ is the ring dispersion, $\varepsilon_{x}$ and $\varepsilon_{y}$ are the rms emittances, $\sigma_{p}$ is the rms relative momentum spread, $<>_{\mathrm{s}}$ denotes averaging along the ring circumference, and $B$ is the bunching factor defined as: $B=\left.(d N / d s)\right|_{\max } /\left(N_{b} q / C\right)$ with $C$ being the ring circumference and $q$ the harmonic number. The painting simulations discussed above result in a particle density in the bunch center which coincides with the particle density of Gaussian beam with horizontal and vertical normalized rms emittances equal to $5 \mathrm{~mm}$ mrad. This value is approximately two times larger than for a Gaussian beam with the same $95 \%$ emittance, i.e. $16 \mathrm{~mm}$ mrad (corresponding rms emittance is equal to $16 / 6 \approx 2.7 \mathrm{~mm} \mathrm{mrad}$ ). Consequently, it decreases the space charge tune shifts by about two times. Figure 2.49 presents the dependence of the space charge tune shifts on time within accelerating cycle. As one can see the space charge tune shifts do not exceed 0.2 .

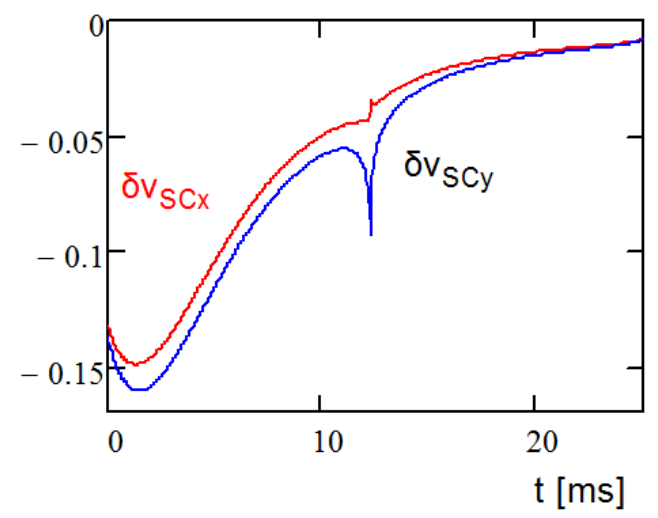

Figure 2.49: The betatron tune shifts due to beam space for horizontal and vertical planes within accelerating cycle. The reduction of tune shifts due to non-Gaussian shape of the particle distribution (see details in Section 2.3.2.3) is taken into account.

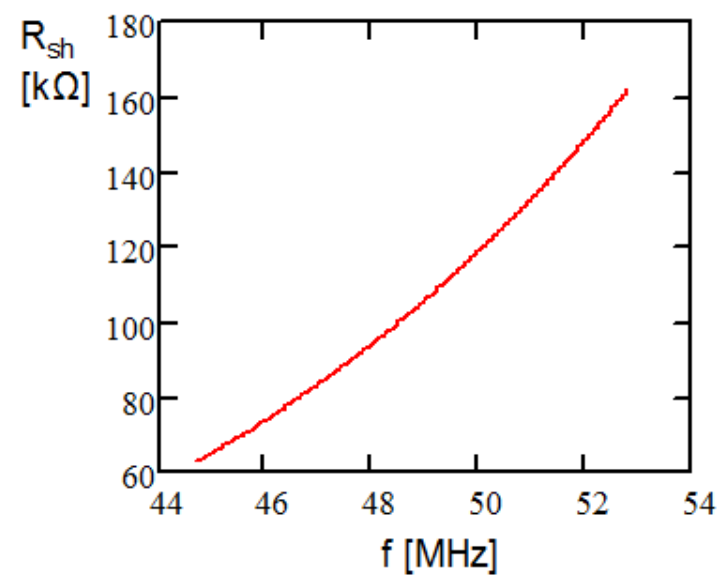

Figure 2.50: The shunt impedance of the Booster RF cavity in the frequency range required for PIP-II operation.

For the present RF system the beam-induced voltage significantly exceeds the RF system voltage required for beam acceleration and capture. Figure 2.50 presents the measured shunt impedance of the present Booster cavities, and Figure 2.51 the beam induced voltage and corresponding powers. As one can see, at the RF voltage maximum, the beam induced voltage (at 
resonance) exceeds the required RF voltage by about 2 times. This ratio achieves its maximum of about 30 at the end of the accelerating cycle.

Note that the power loss in the cavity walls presented in Figure 2.51 assumes equal voltage distribution in all cavities and their perfect phasing. A more practical way of obtaining small RF voltages in the presence of large beam current is paraphasing of two groups of cavities. This technique is presently used in the Booster and will be used in the future for PIP-II. In this case a small voltage is achieved by operating two groups of cavities with comparatively large and equal voltages with an RF phase difference close to $180 \mathrm{deg}$. That implies that power loss in the cavity walls will be significantly larger at the cycle beginning and the cycle end, where the RF voltage is small, than the value presented in Figure 2.51.

Power/cavity, $\mathrm{kW}$

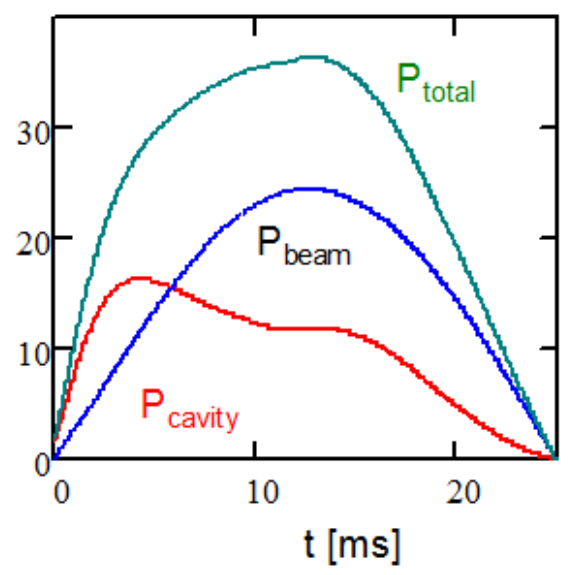

Beam induced RF voltage/cavity

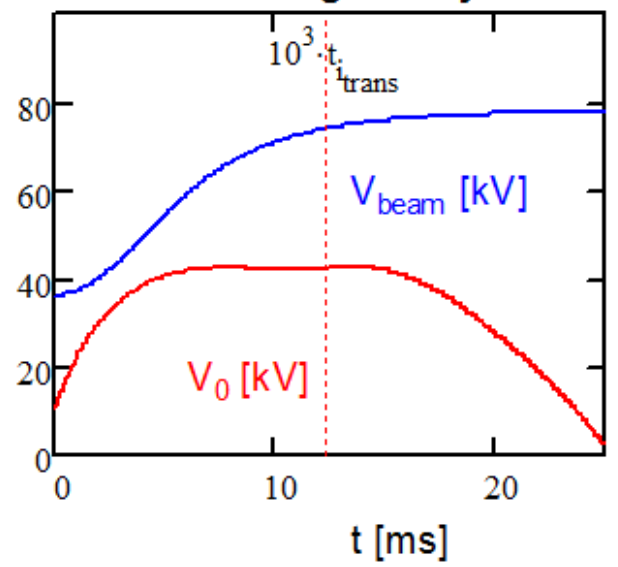

Figure 2.51: Left pane: dependences on time within accelerating cycle for the power loss in the cavity walls (red, $P_{\text {cavity }}=V_{0}^{2} / / R_{s h}$ ), beam power transferred to the beam (blue) and total RF power to the cavity (green). Right pane: dependences on time within accelerating cycle for the total RF voltage per cavity (red) and the beam induced voltage. 20 accelerating cavities are implied. Voltage jumps at transition are not shown.

An additional reduction of the space charge betatron tune shifts can be achieved with a double harmonic RF system. In this case the voltages of the fundamental (first harmonic) RF system and an additional RF system operating at the second harmonic can create a longitudinal potential well with a flat bottom resulting in a reduction of the bunching factor. The installation of the second harmonic RF system is planned for the presently proceeding Proton Improvement Plan (PIP). Note that relative misphasing of the first and second harmonic RF systems deteriorates the flat bottom of the potential well. That results in an increase of longitudinal density and, subsequently, leads to a particle loss. The required accuracy of relative phasing is about $5 \mathrm{deg}$. of the first harmonic frequency. Addressing the voltage stability and relative phasing will require a sophisticated low level RF.

\subsubsection{Booster Longitudinal Impedance}

To exclude the eddy currents excited in a vacuum chamber by fast changing magnetic field the Booster does not have a vacuum chamber in the usual sense of this word, rather its beam aperture is formed by poles of laminated combined function dipoles. That greatly amplifies its longitudinal impedance. 
To estimate the longitudinal impedance of such "laminated" beam aperture we use the model considered in Ref. [41] which derives the longitudinal impedance of flat laminated dipole with constant gap between poles. The chamber geometry is presented in Figure 2.52. The results of Ref. [41] (see Eqs. (5.12) and (5.19) in there) can be rewritten in the following form, presenting the longitudinal impedance per unit length as a function of frequency:

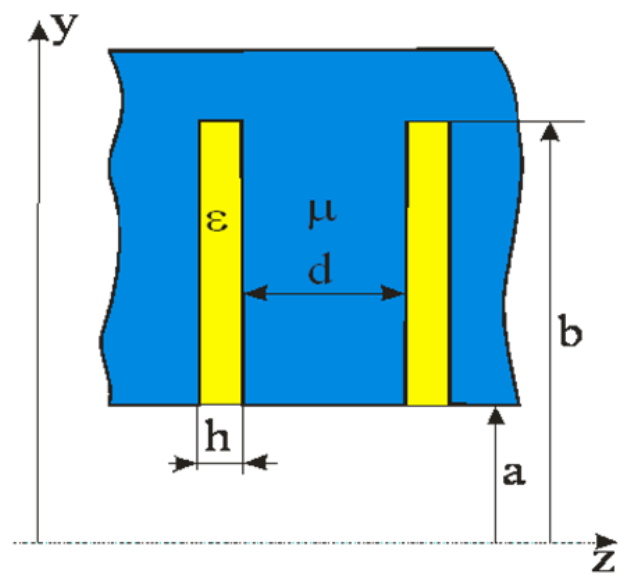

Figure 2.52: Geometry of the laminated beam aperture (or "vacuum chamber"). The beam moves in the $\mathrm{z}$-direction. The chamber is infinite in $\mathrm{x}$-direction (normal to the picture plane).

$$
Z_{\|_{L M}}(\omega)=i Z_{0} \frac{\omega}{2 \pi c} \int_{0}^{\infty} \frac{F_{L}(\xi)}{1+F_{L}(\xi) \tanh \xi} \frac{d \xi}{\xi \cosh ^{2} \xi},
$$

where

$$
\begin{aligned}
& F_{L}(\xi)=\frac{h}{d+h} \frac{\xi}{k_{y}(\xi)}\left(1+(1-i) \frac{\mu \delta_{S}}{h}\right) \tan \left(k_{y}(\xi)\left(\frac{b}{a}-1\right)\right), \\
& k_{y}(\xi)=\sqrt{\frac{\varepsilon \omega^{2} a^{2}}{c^{2}}\left(1+(1-i) \frac{\mu \delta_{S}}{h}\right)-\xi^{2}},
\end{aligned}
$$

$\mathrm{Z}_{0}=4 \pi / \mathrm{c} \approx 377 \Omega, c$ is the light velocity, $a$ is the half-gap between dipole poles, $(b-a)$ is the depth of laminations, $\varepsilon$ is the effective dielectric constant of the filling (epoxy plus insulating oxide layer), $h$ is the distance between laminations ${ }^{5}, \delta_{S}=c / \sqrt{2 \pi \sigma_{R} \omega \mu}$ is the skin depth, $\sigma_{R}$ is the steel conductivity, and $\mu$ is the steel permeability. At frequencies of interest the skin depth is smaller than, or about the same as, the magnetic domain size, which greatly reduces the magnetic permeability and makes it complex. For an estimate we use the measured magnetic permeability of soft steel presented in Ref. [42]. The measurements for the intermediate values of magnetic field were fitted to a simple expression which plot is presented in Figure 2.53. Other parameters are presented in Table 2.18. The steel conductivity was taken from Ref. [42]. Results of the numerical integration of Eq. (2.9) are presented in Figure 2.54. We assume here that the distance between laminations is constant, while in reality it is changing within each gap and from gap to gap in some uncontrolled way. Consequently, Eq. (2.10) has a quite limited accuracy and measurements of the dipole impedance are highly desirable. As shown in Ref [42] the high frequency magnetic permeability, the same as for DC case, depends on the magnetic field in a dipole. That results in a

\footnotetext{
${ }^{5}$ The distance between laminations was estimated from the known packing factor.
} 
dependence of the dipole impedance on its magnetic field.

Two types of measurements were used. The first method is based on the stretched wire measurements [43] allowing measurements of a single dipole impedance, and the second one on a shift of accelerating phase with beam intensity allowing an indirect measurement of the entire Booster impedance.

Table 2.18: Parameters of laminations used for the impedance estimates of the Booster laminated dipoles

\begin{tabular}{l|c|c|l}
\hline \hline Dipole type & $\mathrm{F}$ & $\mathrm{D}$ & \\
\hline Dipole length & \multicolumn{2}{|c|}{2.89} & $\mathrm{~m}$ \\
\hline Number of dipoles & 48 & 48 & $\mathrm{~cm}$ \\
\hline Half-gap, $a$ & 2.1 & 2.9 & $\mathrm{~cm}$ \\
\hline Lamina half-height, $b$ & \multicolumn{2}{|c|}{15.2} & $\mathrm{~cm}$ \\
\hline Lamina thickness, $d$ & \multicolumn{2}{|c|}{0.64} & $\mathrm{~mm}$ \\
\hline Dielectric crack width, $h$ & \multicolumn{2}{|c|}{20} & $\mu \mathrm{m}$ \\
\hline Conductivity, $\sigma$ & $2.07 \cdot 10^{16}\left(2.3 \cdot 10^{6} \Omega^{-1} \mathrm{~m}^{-1}\right)$ & $\mathrm{s}^{-1}$ \\
\hline Dielectric permittivity, $\varepsilon$ & \multicolumn{2}{|c|}{4.75} & \\
\hline \hline
\end{tabular}

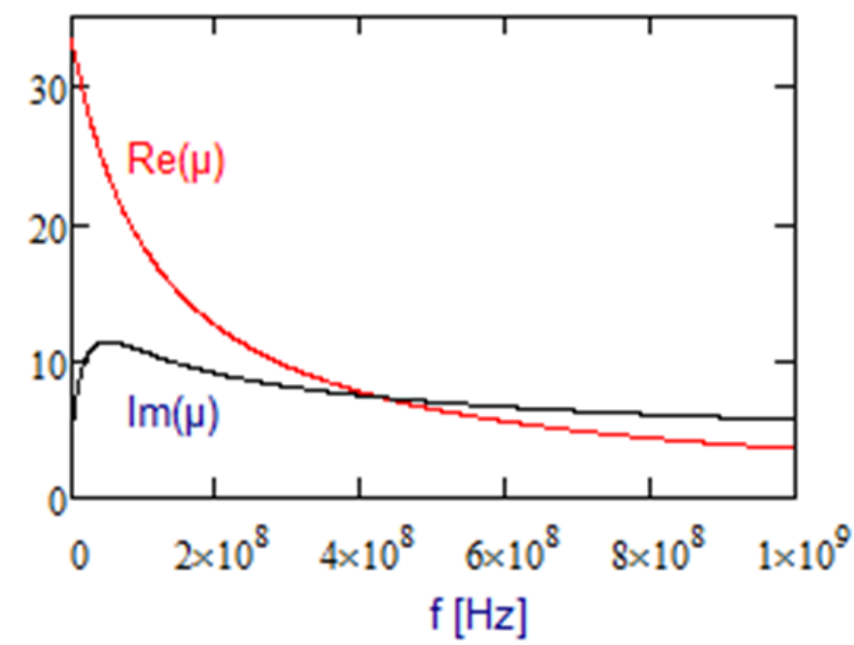

Figure 2.53: The dependence of magnetic permeability on frequency used for computation of the Booster longitudinal impedance.

Figure 2.55 presents results of the longitudinal impedance measurements for two spare Booster dipoles [43] performed with the stretched wire. As on can see the measurements and the calculations are in a reasonable agreement for F-dipole. However an agreement is much worse for D-dipole. It is important to note that the measured impedance of the D-dipole is larger than that of the F dipole while theory predicts the opposite - the dipole with larger aperture should have smaller impedance. Most probably it is related to a difference in details of lamination packing in these two dipoles. Thus, we should expect that each dipole has its unique impedance. Consequently, beam based measurements of Booster impedance is the only reliable way to obtain the Booster impedance. 


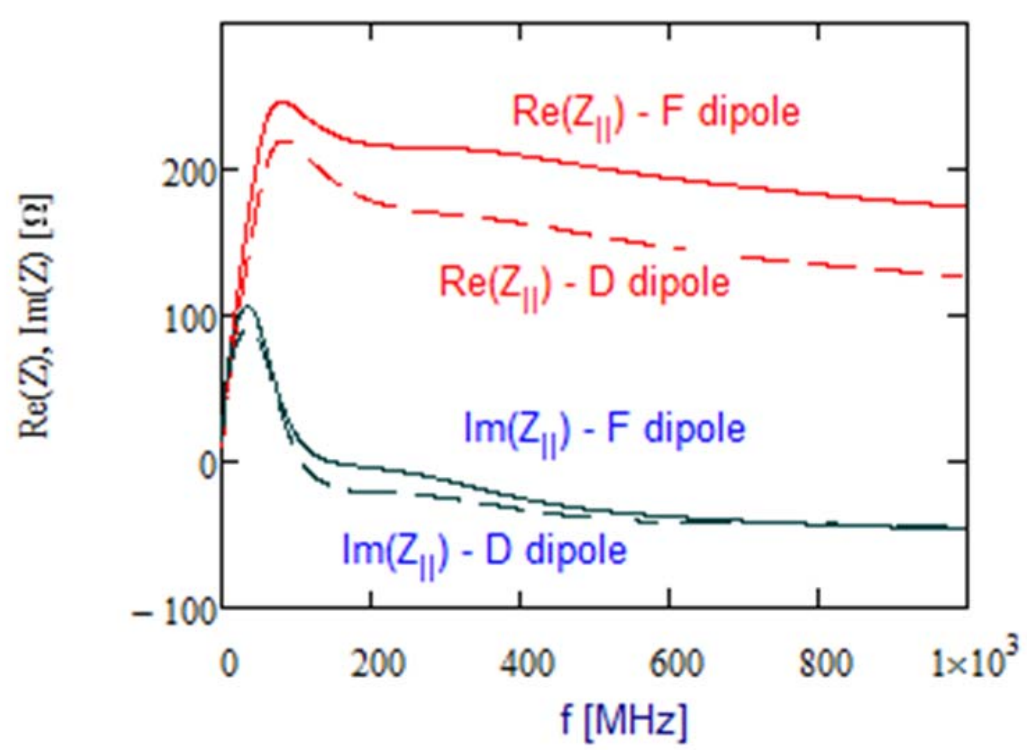

Figure 2.54: The dependences of longitudinal impedance on frequency computed with Eq. (2.9) for the Booster $\mathrm{F}$ and $\mathrm{D}$ dipoles.
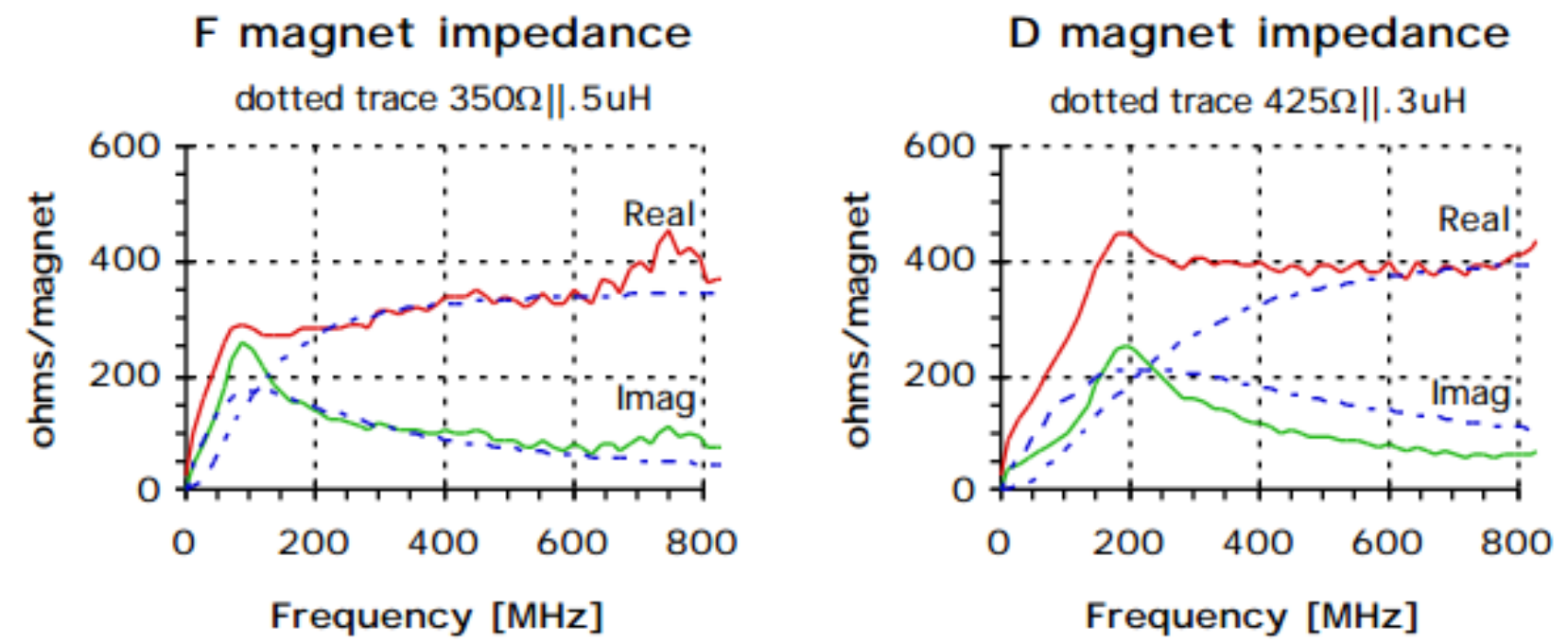

Figure 2.55: Dependence of longitudinal impedance of Booster dipole on the frequency measured with the stretched wire method for F and D dipoles [43]. 


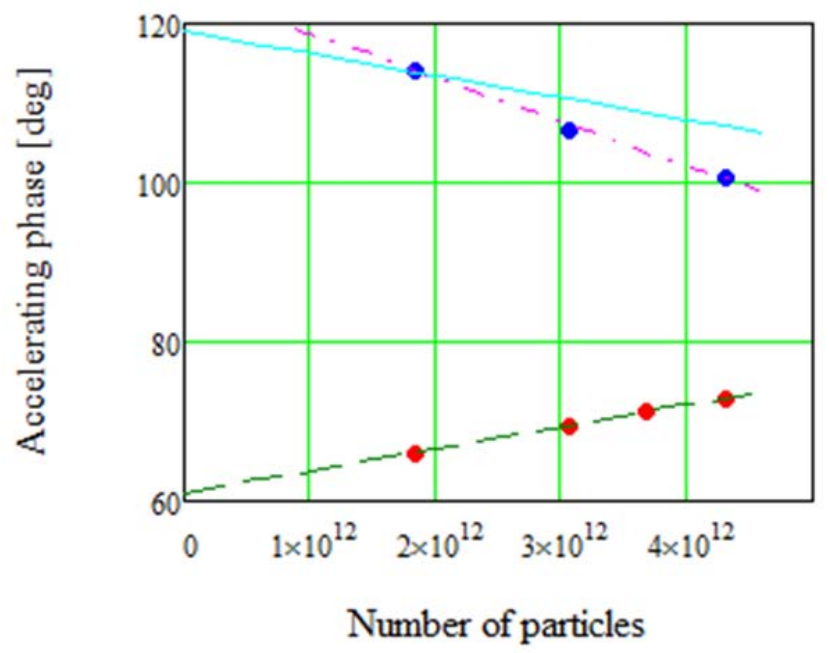

Figure 2.56: The dependence of accelerating phase on the number of particles in the beam before (red dots) and after (blue dots) transition; 82 bunches in 84 RF buckets: rms bunch length of 0.75 $\mathrm{ns}, \mathrm{RF}$ voltage at transition of $670 \mathrm{kV}$ and the accelerating phases for zero current before and after transition are 61 and 119 deg., respectively.

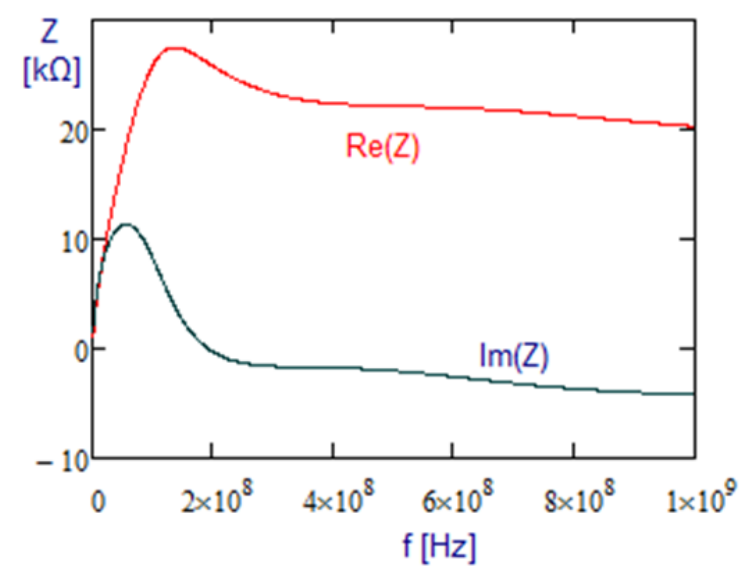

Figure 2.57: The contribution of Booster laminated dipoles to its longitudinal impedance; based on the theoretical model of Eq. (2.9) with $\varepsilon_{d}=2.3$ and other parameters presented in Table 2.18.

The direct beam-based measurements of the effective Booster impedance were based on the measurements of the accelerating phase shift with beam current [44]. The data was acquired in the vicinity of transition crossing and only data before the transition were used, because the phase manipulations at transition result in an additional intensity dependent energy loss, consequently, yielding a steeper dependence of the phase shift on the intensity after transition. The measurement results are presented in Figure 2.56. They yield the phase shift of $11.9 \mathrm{deg}$. for the beam intensity of $4.3 \cdot 10^{12}$ while the theoretical estimate considered above predicts $9.9 \mathrm{deg}$. The $20 \%$ difference looks as a quite good coincidence taking into account a poor knowledge of the parameters used in the theoretical estimate. For the transition crossing simulations considered below we decrease the dielectric permittivity from 4.75 to 2.3 (see Table 2.18). It results in only minor changes in the shapes of the impedance curves but increases the impedance by about $20 \%$ resulting good coincidence between the prediction based on the impedance model and the measurements. Figures 
2.57 and 2.58 present the corresponding contribution from the Booster dipoles to the entire Booster impedance and the corresponding decelerating voltage for the nominal PIP-II beam intensity and bunch length at the transition crossing. As one can see the peak of deceleration is achieved near beam center and achieves $140 \mathrm{kV} /$ turn.

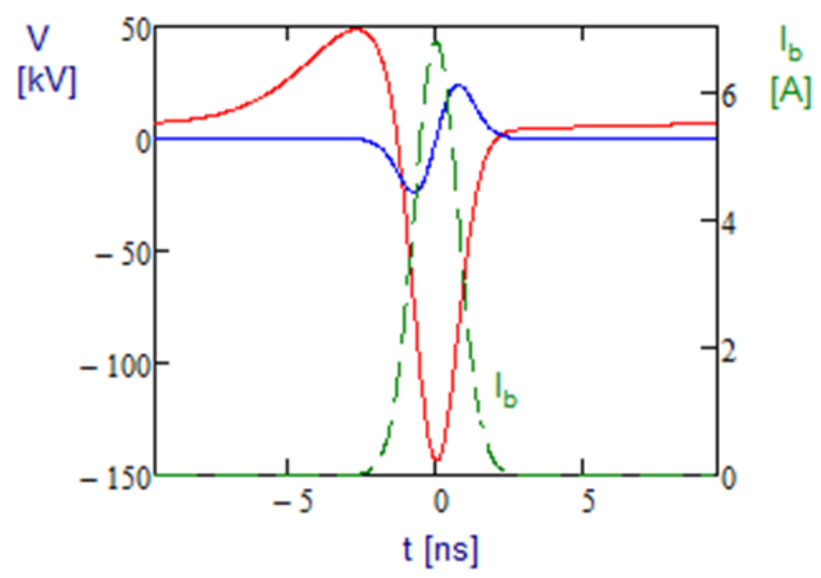

Figure 2.58: The beam induced voltage due to impedances of the laminated dipoles (red) and the space charge (blue) for $6.5 \cdot 10^{12}$ particles and rms bunch length of $0.75 \mathrm{~ns}$; the bunch head is at positive coordinates, effect of preceding bunches is accounted. The green dashed line shows the bunch current.

The longitudinal impedance due to bunch space charge is:

$$
Z_{\|_{s c}}(\omega) \approx-i Z_{0} \frac{\omega}{\beta \gamma^{2} \omega_{0}} \ln \left(\frac{r_{c}}{1.06 \sigma_{t r}}\right), \frac{r_{c}}{\sigma_{\perp}} \geq 2,
$$

where $\beta$ and $\gamma$ are the beam relativistic factors, $\omega_{0}$ is the revolution frequency, $r_{c}$ is the vacuum chamber radius and $\sigma_{\perp}$ is the rms transverse beam size. It diminishes fast with beam acceleration however, as will be seen in the next chapter, it makes the major contribution to the longitudinal emittance growth excited by transition crossing. Figure 2.58 compares the beam space charge induced voltage to the voltage excited by resistivity of dipole laminations. Other sources of the impedance are small and can be safely omitted in simulations of beam acceleration.

\subsubsection{Transition crossing}

The longitudinal force of the beam space charge changes its "sign" at transition, in the sense that the particle repulsion before the transition is replaced by particle attraction after it. This results in longitudinal quadrupole oscillations leading to the longitudinal emittance growth. Presently, a longitudinal quadrupole damper is used to suppress these oscillations. It keeps longitudinal emittance growth at manageable level. This effect will be much more pronounced with higher intensity. To prevent the longitudinal emittance growth a faster suppression of quadrupole motion is required. Taking into account that the process is repeatable we plan to add a powerful feedforward system to the existing quadrupole damper. It will suppress quadrupole oscillations much faster and, consequently, will result in significantly smaller longitudinal emittance growth. Figure 2.59 shows how two RF voltage bumps suppress the quadrupole oscillations after transition. This technique is called the voltage jump technique. Another option that was considered is a $\gamma_{t}$ jump. However simulations show that it is significantly less effective that the voltage jump. 

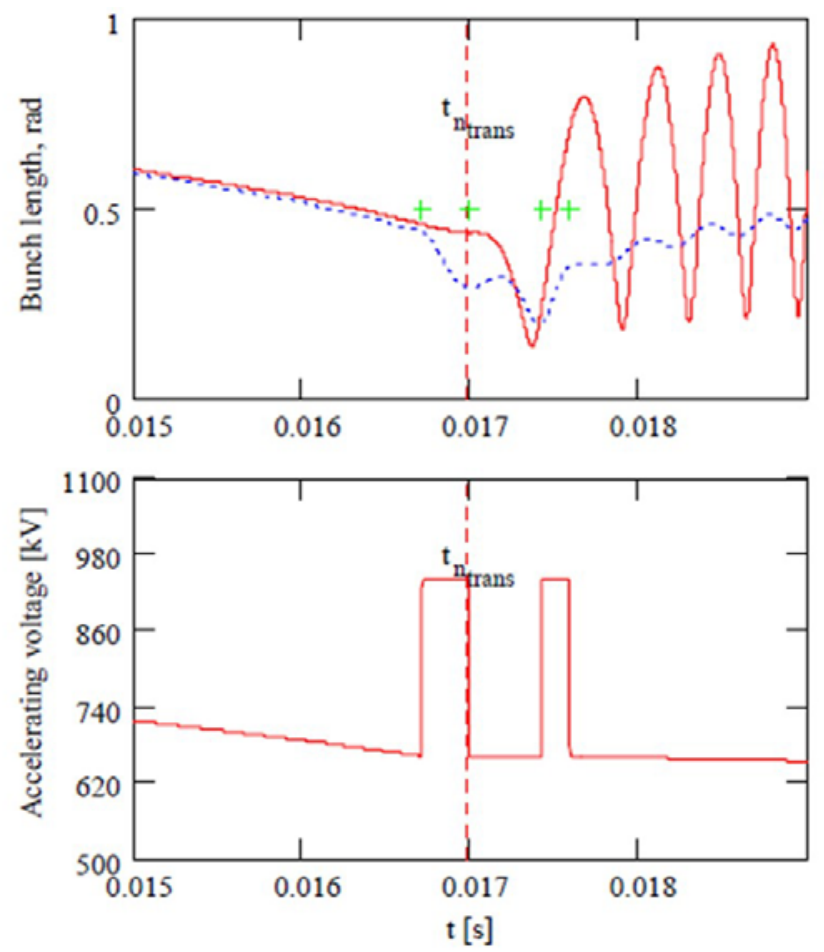

Figure 2.59: Voltage jumps to control quadrupole oscillations at transition. The upper figure shows quadrupole (bunch length) oscillations following transition without (red) and with (blue) the RF voltage jumps shown in the lower figure.

\subsubsection{Modifications to the Magnet System Required for $20 \mathrm{~Hz}$ Operation}

The present system has 96 magnets in a 24 cell arrangement (see Figure 2.60). These are driven by four power supplies that are the MR (Main Ring) style, $720 \mathrm{~Hz}$ update rate SCRs. Regulation is based on magnetic field measurements in a reference magnet with B-dot coil and transductor electronics. A sinusoidal drive signal excites the resonant system with a quality factor of about 40 for $15 \mathrm{~Hz}$ operation. Corrections for losses and line voltage variations are done by a card in a VXI crate. Regulation is good to about a part in 4000 .

The conversion of GMPS (Gradient Magnet Power Supply) controls from 15 to $20 \mathrm{~Hz}$ does not look difficult. The system was designed to run at $10 \mathrm{GeV}$ and therefore it is capable to operate at higher voltage and power compared to the present $8 \mathrm{GeV}, 15 \mathrm{~Hz}$ operation.

To verify that the Booster dipoles can operate at $20 \mathrm{~Hz}$ an experimental test was carried out [45]. Measurements were performed on both a Booster gradient magnet and a Booster choke with the intent to compare the $15 \mathrm{~Hz}$ losses with the $20 \mathrm{~Hz}$ losses for the proposed Booster upgrade. The analysis carried out after the measurements suggests that running the Booster at $20 \mathrm{~Hz}$ with magnet current ramping in the same range as for the present operation will require about $3.9 \%$ more power. To increase the resonant frequency the resonant capacitor at each "Girder" must decrease from $\sim 8.33 \mathrm{mF}$ to $\sim 4.69 \mathrm{mF}$. As result the capacitor voltage will increase by about $32 \%$ with a subsequent slight increase in the rms current for the choke, magnets and capacitors. This also implies that the rms current per $\mu \mathrm{F}$ will increase as well. The girder drive voltage will increase by about $9.2 \mathrm{~V}(\mathrm{p}-\mathrm{p})$. The present magnet power system runs on 4 power supplies but can operate with only 3 supplies. Booster at $20 \mathrm{~Hz}$ would require all 4 PS to operate. 


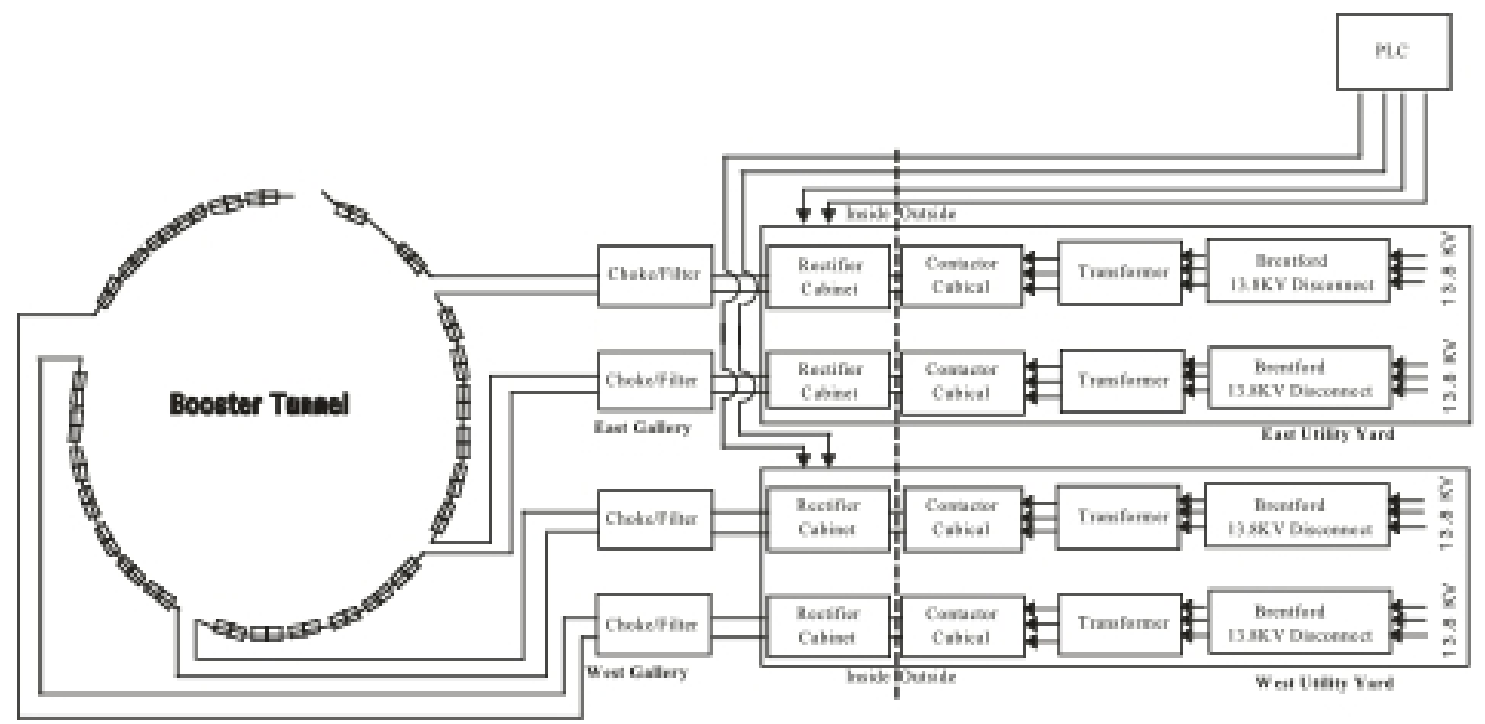

Figure 2.60: Schematic of the Booster magnets power system.

\subsubsection{Beam Instabilities}

The main challenges in achieving beam stability in the Booster are associated with transverse instabilities at the injection and transition. The longitudinal instability at transition is considered elsewhere [46] and is not discussed below. Table 2.19 provides the main beam parameters used in the following estimates.

The Booster wide band impedances are known to be dominated by impedances of the laminated magnets. The corresponding equations were derived in Ref. [47, 48]. The magnet parameters used for the impedance calculations are presented in the Table 2.20.

The transverse impedance and wake function for a round laminated chamber (magnet) with the radius $a=2.1 \mathrm{~cm}$ are presented in Figures 2.61 and 2.62. The wake functions for the horizontal and vertical degrees of freedom can be approximated by accounting for the Yokoya factors equal to 0.4 and 0.8 , respectively. The gaps for $\mathrm{F}$ and $\mathrm{D}$ dipoles are different. That makes the wakes of the defocusing magnet approximately two times smaller than for the focusing one. Accounting for the filling factor (fraction of the orbit taken by magnets), $\eta_{m}=0.58$, leads to the machine weighted horizontal and vertical wakes:

$$
\begin{aligned}
& \bar{W}_{x}(s)=0.4 W_{1}(s) C\left(\frac{\beta_{x F}}{\bar{\beta}}+0.5 \frac{\beta_{x D}}{\bar{\beta}}\right) \frac{\eta_{m}}{2} \equiv q_{x} \eta_{m} W_{1}(s) C, \\
& \bar{W}_{y}(s)=0.8 W_{1}(s) C\left(\frac{\beta_{y F}}{\bar{\beta}}+0.5 \frac{\beta_{y D}}{\bar{\beta}}\right) \frac{\eta_{m}}{2} \equiv q_{y} \eta_{m} W_{1}(s) C .
\end{aligned}
$$

Here $C=2 \pi R$ is the machine circumference, $\beta_{x F}$ and $\beta_{x D}$ are the horizontal beta-functions averaged over focusing and defocusing magnets and similar $\beta_{y F}$ and $\beta_{y D}$ for the vertical betafunctions. and $\bar{\beta}$ is the average ring beta-function ${ }^{6}$. Substituting numerical values one obtains: $q_{x}$ $\approx 0.50$ and $q_{y} \approx 0.45$.

\footnotetext{
${ }^{6}$ The same $\bar{\beta}$ is used in the instability equations and will be cancelled in the final result, i.e. this value is used for normalization, and its exact value is irrelevant for the final result.
} 
Table 2.19: Beam parameters used in estimates of Booster instabilities

\begin{tabular}{l|c|l}
\hline \hline & Requirement & \\
\hline Bunch population, $N$ & $8.3 \cdot 10^{10}$ & \\
\hline Transverse emittance, norm. rms, $\varepsilon_{n \perp}$ & 2.7 & $\mathrm{~mm} \cdot \mathrm{mrad}$ \\
\hline Longitudinal emittance, $\mathrm{rms}, \varepsilon_{\|}=\sigma_{\mathrm{z}} \sigma_{\mathrm{E}}$ & 3.2 & $\mathrm{meV} \cdot \mathrm{s}$ \\
\hline Maximal RF Voltage, $V$ & 0.75 & $\mathrm{MV}$ \\
\hline Maximal acceleration rate, $\dot{\gamma}$ & 0.5 & $\mathrm{~ms}^{-1}$ \\
\hline Transition gamma, $\gamma_{t}$ & 5.47 & \\
\hline \hline
\end{tabular}

Table 2.20: Magnet parameters

\begin{tabular}{l|l|l}
\hline \hline Half-gap F/D, $a$ & $2.1 / 2.9$ & $\mathrm{~cm}$ \\
\hline Lamina thickness, $d$ & 0.64 & $\mathrm{~mm}$ \\
\hline Dielectric crack width, $h$ & 20 & $\mu \mathrm{m}$ \\
\hline Conductivity, $\sigma$ & $4.5 \cdot 10^{16}$ & $\mathrm{~s}^{-1}$ \\
\hline Dielectric permittivity, $\varepsilon$ & 4.75 & \\
\hline Magnetic permeability, $\mu$ & 50 & \\
\hline Average $\beta$-functions, $\beta_{x F}, \beta_{y F}, \beta_{x D}, \beta_{y D}$ & $30,7,11,18$ & $\mathrm{~m}$ \\
\hline \hline
\end{tabular}

For multi-bunch beams with strong space charge [49], the modes are characterized by two indices: the single-bunch (or head-tail, or intra-bunch) index $n$, and the coupled-bunch (or interbunch) index $\mu$. At the first order of the perturbation theory over the wake function, the coherent tune shift $\Delta v_{n \mu}$ of the mode $|n, \mu\rangle$ is proportional to the sum of single- and coupled-bunch diagonal matrix elements of the wake function $[49,50]$ :

$$
\begin{aligned}
& \Delta v_{n \mu}=-\frac{N r_{p} \bar{\beta}\left(\hat{W}_{n}^{\mathrm{SB}}+\hat{W}_{n \mu}^{\mathrm{CB}}\right)}{4 \pi \beta^{2} \gamma} \equiv \Delta v_{n \mu}^{\mathrm{SB}}+\Delta v_{n \mu}^{\mathrm{CB}} ; \\
& \hat{W}_{n}^{\mathrm{SB}}=\iint d s d s^{\prime} \bar{W}\left(s-s^{\prime}\right) \exp \left(i \zeta\left(s-s^{\prime}\right)\right) \rho(s) \rho\left(s^{\prime}\right) y_{n}(s) y_{n}\left(s^{\prime}\right) ; \\
& \hat{W}_{n \mu}^{\mathrm{CB}}=W_{\mu}^{\mathrm{CB}}\left|I_{n}(\chi)\right|^{2} ; I_{n}(\chi)=\int d s \exp \left(i \chi s / \sigma_{s}\right) \rho(s) y_{n}(s) ; \\
& W_{\mu}^{\mathrm{CB}}=\sum_{k=1}^{\infty} \bar{W}(k C / M) \exp \left(i k \psi_{\mu}\right) ; \quad \psi_{\mu}=2 \pi\left(\left\{Q_{b}\right\}+\mu\right) / M ; \quad \mu=0,1, \ldots, M-1 .
\end{aligned}
$$




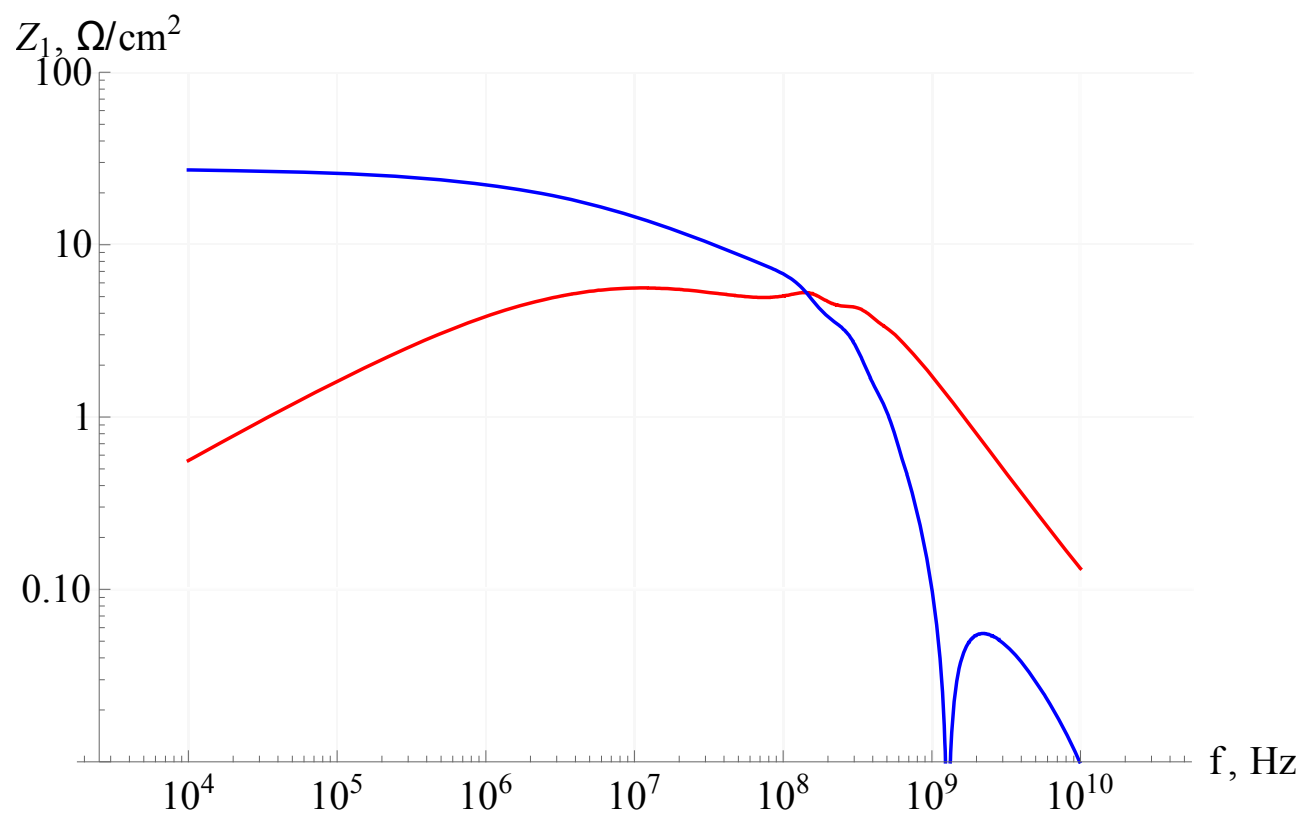

Figure 2.61: Transverse impedance per unit length $(\operatorname{Re} Z, \operatorname{Im} Z)$ of a round laminated magnet with radius $a=2.1 \mathrm{~cm}$.

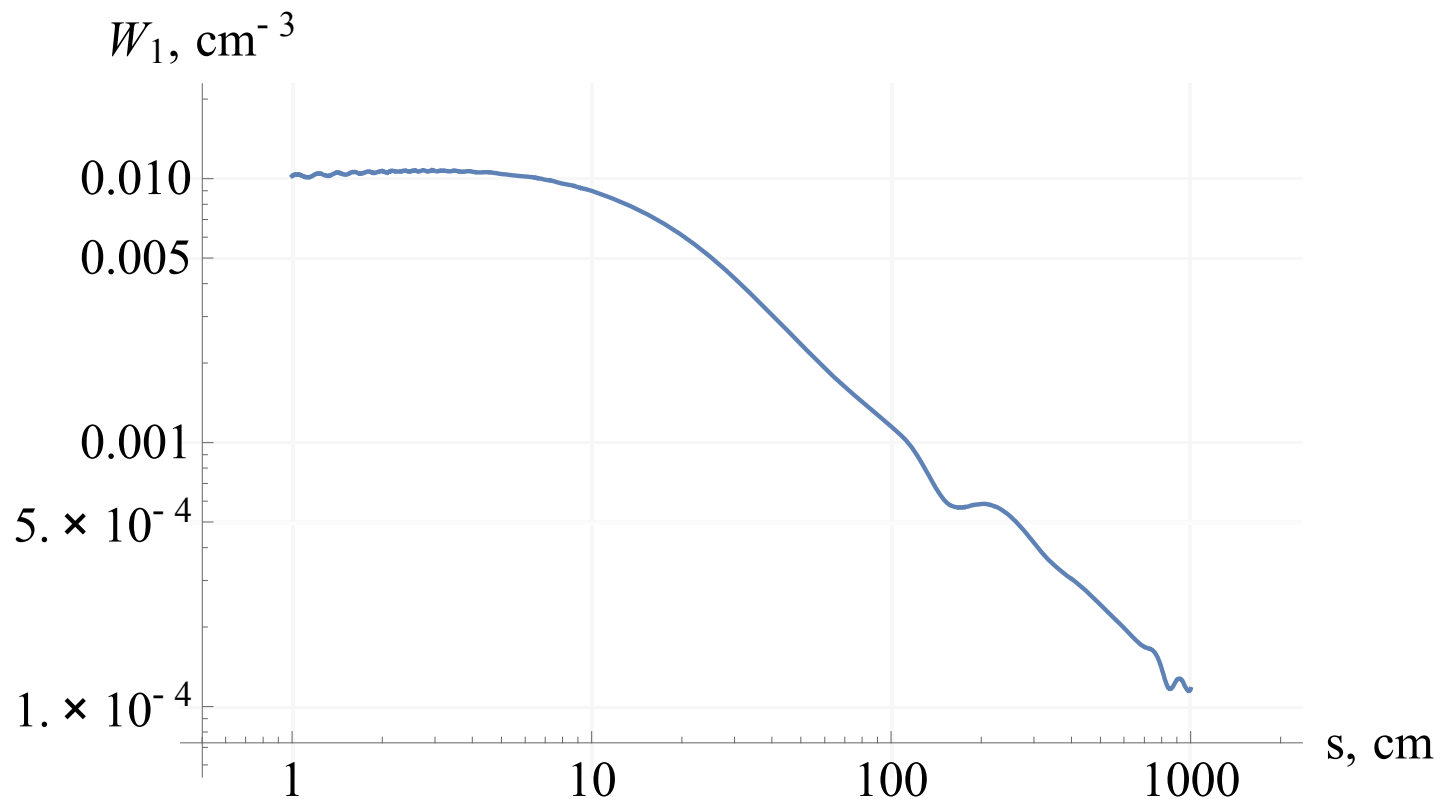

Figure 2.62: The transverse wake function per unit length of the same magnet as in Figure 2.61.

Here $N$ is the number of particles per bunch, $r_{p}$ is the classical radius, $\beta$ and $\gamma$ are the relativistic factors, $\chi=\zeta \sigma_{s}=\xi \sigma_{s} /(R \eta) \equiv \omega_{\xi} \sigma_{\tau}$ is the head-tail phase (see Eq. 6.187, p. 339 in Ref. [51]) with $\xi$ as the chromaticity and $\eta$ as the slip-factor, $\rho(s)$ is the normalized line density, $y_{n}(s)$ is the $n$-th head-tail eigen-function, $M$ is the number of bunches, and $\left\{Q_{b}\right\}$ is a fractional part of the betatron tune. At sufficiently small head-tail phase, the single-bunch growth rate can be neglected, while the coupled-bunch growth time is calculated as $\left[\omega_{0} \operatorname{Im}\left(\Delta v_{00}^{\mathrm{CB}}\right)\right]^{-1}=80 \mu \mathrm{s}$, in agreement with Ref. [52]. Feedback can be taken into account similarly to the coupled-bunch wake [50]; for a bunch-by- 
bunch damper with a gain $g$ this yields the damping rate

$$
\operatorname{Im}\left(\Delta v_{n \mu}\right)=-g\left|I_{n}(\chi)\right|^{2} \text {. }
$$

To make the description complete, the Landau damping has to be taken into account. To find it with good accuracy, the order-of-magnitude estimates of Landau damping suggested in Ref. [49] have to be compared with dedicated tracking simulations, e.g. with the Synergia [53, 53]. Performing this will complete the simulation scheme, yielding accurate predictions and recommendations for various operation scenarios.

At transition, the strong head-tail instability is suppressed by the chromaticity with a threshold value that is proportional to the bunch population. Thus, the increase of the latter by a factor of 1.5 compared with the current value would require a similar increase of the former. 


\subsection{Recycler and Main Injector Modifications}

\subsubsection{Technical Requirements and Scope}

The performance requirements of the Main Injector/Recycler complex are summarized in Table 2.21. The Recycler has recently been reconfigured as a proton accumulation ring in support of the NOvA experiment. For PIP-II an increase in beam intensity of $50 \%$ over current operations is required. It will be accompanied by a modest (10\%) decrease in the Main Injector cycle time for $120 \mathrm{GeV}$ operation. The primary requirement on the Recycler is to slip-stack twelve Booster batches and to deliver this accumulated beam to the Main Injector in a single turn. In order to maintain losses at current levels the efficiency of this operation has to be at least $97 \%$.

Table 2.21: Main Injector/Recycler requirements for 0.9-1.2MW operations at 60-120GeV

\begin{tabular}{l|c|l}
\hline \hline Performance Parameter & Requirement & \\
\hline Particle Species & Protons & \\
\hline Injection Beam Energy (kinetic) & 8.0 & $\mathrm{GeV}$ \\
\hline Extracted Beam Energy (kinetic) & $60-120^{*}$ & $\mathrm{GeV}$ \\
\hline Protons per Pulse (injected) & $7.7 \times 10^{13}$ & \\
\hline Protons per Pulse (extracted) & $7.5 \times 10^{13}$ & \\
\hline Slip-stacking Efficiency & 97 & $\%$ \\
\hline Controlled 8 GeV losses to Abort & 0.8 & $\%$ \\
\hline Controlled 8 GeV losses to Collimators & 1.7 & $\%$ \\
\hline Uncontrolled 8 GeV losses & 0.5 & $\%$ \\
\hline Transition Losses & 0.2 & $\%$ \\
\hline Cycle Time & $0.8-1.2$ & $\mathrm{sec}$ \\
\hline Beam Power & $0.9-1.2$ & $\mathrm{MW}$ \\
\hline Beam Emittance (95\%, normalized) & 20 & $\mathrm{~mm}-\mathrm{mrad}$ \\
\hline Bunching Factor & 0.5 & \\
\hline Laslett Tune Shift (Injection) & -0.06 & \\
\hline \hline
\end{tabular}

* The Main Injector is capable of maintaining beam power of $1.2 \mathrm{MW}$ for energies as low as $80 \mathrm{GeV}$.

In order to provide the RF power required to accelerate $7.5 \times 10^{13}$ protons three options could be considered:

1. Operate the current RF cavities with two power tubes instead of one in a push-pull configuration. This will require doubling of the number of modulators and solid state drivers.

2. Use a new more powerful power tube, such as the EIMAC 4CW250,000B. This will require a new mounting configuration (to accommodate the much longer tube), new modulators, and upgraded power amplifier cooling.

3. Replace the entire RF system with a new one (new cavities and PAs). The advantage of this solution is that it can accelerate enough intensity to reach $2.3 \mathrm{MW}$ in the next round of Accelerator complex upgrade. 
Options 1 and 2 will be considered for PIP-II in more details as they are substantially less expensive than Option 3.

\subsubsection{Slip-stacking in Recycler}

To be ready for the next Booster injection in time, the beam separation for slip stacking in the Recycler has to be $1680 \mathrm{~Hz}$ or $32 \mathrm{MeV}$ (one Booster batch slippage in one Booster tick). The figure of merit in slip stacking is the parameter alpha that relates the frequency separation in synchrotron frequency unit $\left(f_{s}\right)$ to the energy separation in bucket height unit $\left(H_{B}\right)$ :

$$
\alpha \equiv \frac{\Delta f}{f_{s}}=2 \frac{\Delta E}{H_{B}}
$$

For $\alpha=2$, the hypothetical independent buckets overlap 50\% in energy, and the single particle motion is chaotic everywhere within them. The case of $\alpha=4$ gives tangent boundaries for the hypothetical buckets and in the case of $\alpha=8$ there is space for a complete empty bucket between the upper and lower hypothetical buckets. In practice we have found that a value of $\alpha$ greater than 5 is adequate. Note that for the given frequency separation further increase of $\alpha$ does not yield larger RF bucket size (area of stable motion). A plot of $\alpha$ as a function of the RF voltage for $1680 \mathrm{~Hz}$ separation is shown in Figure 2.63. From that figure we can see that an RF voltage of $140 \mathrm{kV}$ meets our requirements.

The choice of RF voltage determines the area of longitudinal phase space where particles will survive sufficiently long time. Figure 2.64 shows the particles in the initial matching beam contours that survive after $300 \mathrm{msec}$. The largest beam contour with no particle loss corresponds to an emittance of $0.10 \mathrm{eV}$-sec $( \pm 4.2 \mathrm{nsec}, \pm 8.0 \mathrm{MeV})$. If we want slip stacking with $97 \%$ efficiency then $97 \%$ of particles from the Booster should be included in that matching contour.

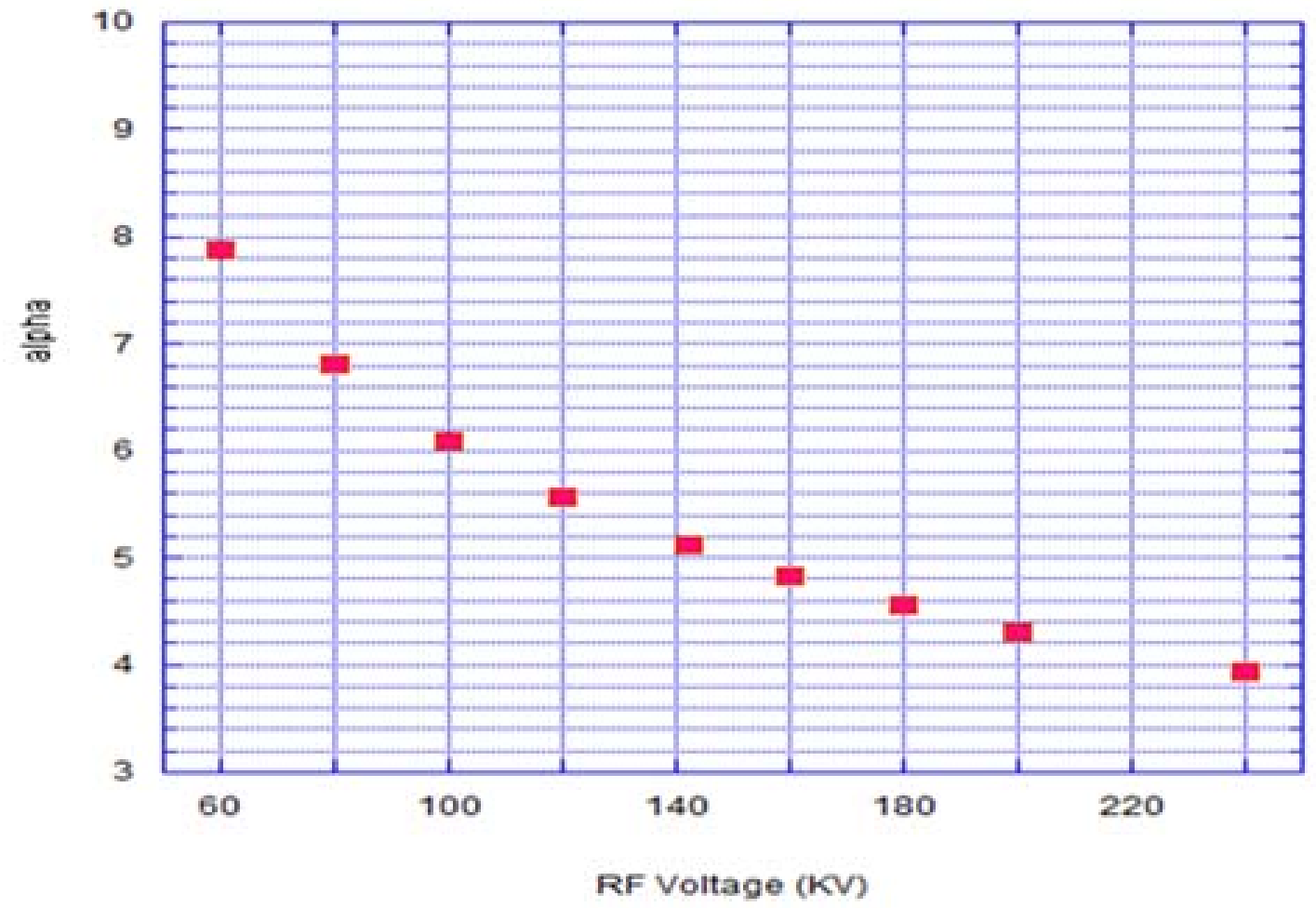

Figure 2.63: $\alpha$ vs. RF voltage for $1680 \mathrm{~Hz}$ separation. 


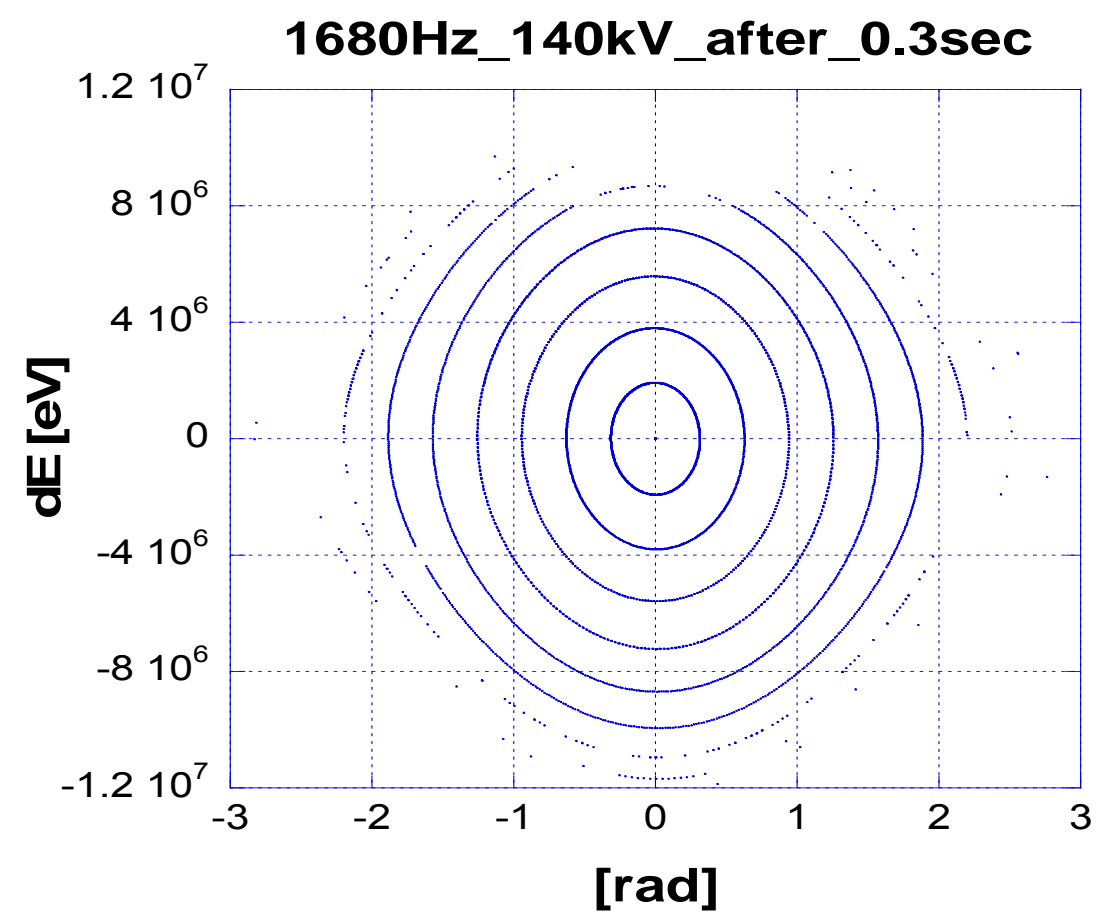

Figure 2.64: Particles on initial matching contours in a $140 \mathrm{KV}$ bucket after $300 \mathrm{~ms}$ of slip stacking with $1680 \mathrm{~Hz}$ separation.

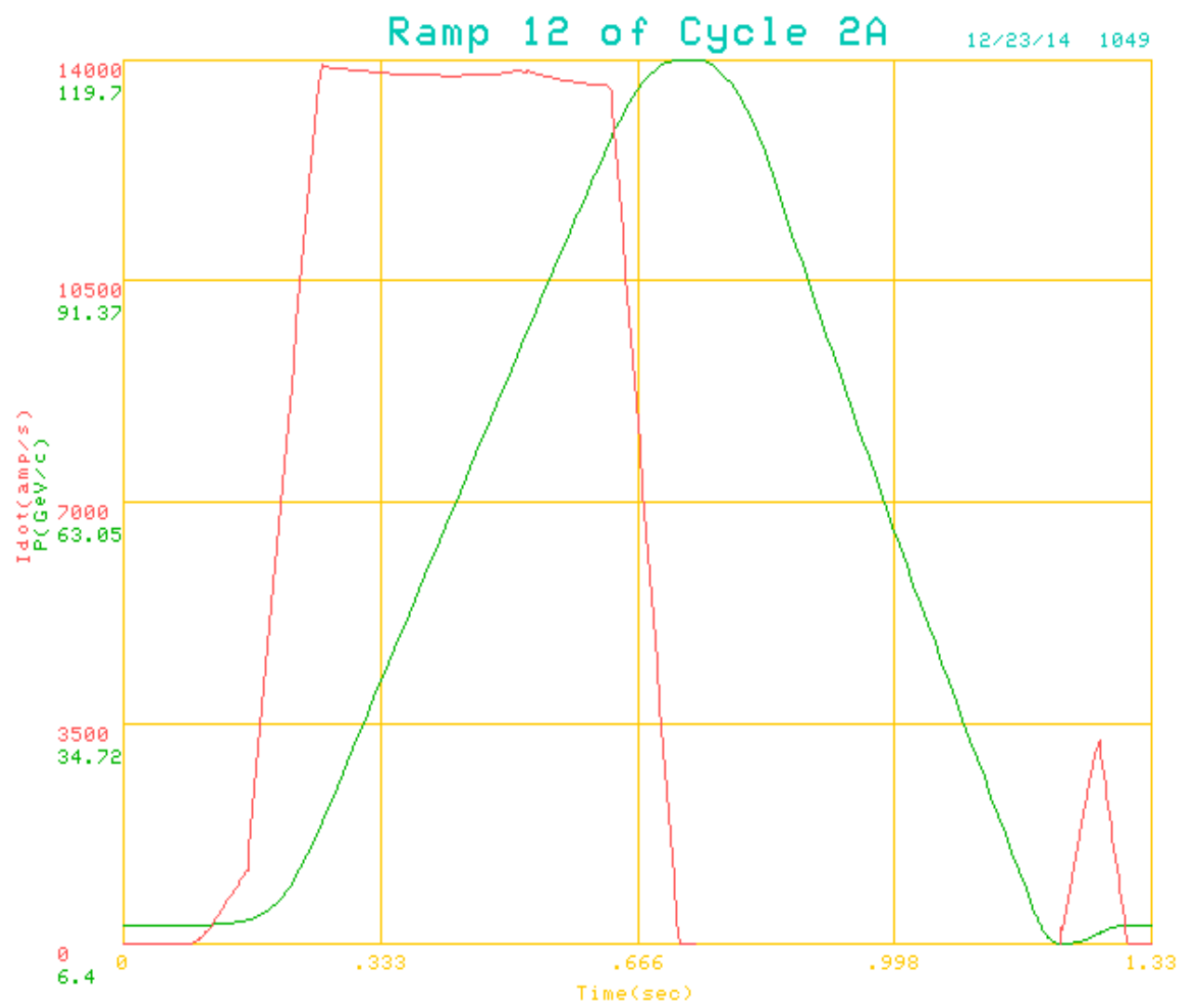

Figure 2.65: The current MI accelerating cycle. The momentum and the rate of dipole current change (acceleration rate) vs time are shown. 


\subsubsection{Acceleration in the MI}

The current (NOvA) MI Acceleration cycle is shown in Figure 2.65. The total cycle duration is $1.33 \mathrm{~s}$. The cycle time can be reduced to $1.2 \mathrm{~s}$ by optimizing the 8.9 and $120 \mathrm{GeV}$ dwell times. In the same figure the acceleration rate, Idot, vs time is also plotted. From the conversion coefficient of $57.754 \mathrm{~A} / \mathrm{GeV}$ we can see that the maximum acceleration rate is $240 \mathrm{GeV} / \mathrm{s}$. The MI RF has to to provide enough voltage to support the above acceleration rate and also to provide the required bucket area. From operational experience with slipped stacked beam a bucket area of at least 2.0 $\mathrm{eV} \cdot \mathrm{s}$ is required above transition to contain the beam tails and avoid losses. The total RF voltage of 4.4 $\mathrm{MV}$ is needed to accelerate the beam and provide the required bucket area above transition. Since the maximum operational voltage from each RF station is $240 \mathrm{kV}$, all $18 \mathrm{RF}$ stations originally installed in the MI are required. During the NOvA shutdown two spare MI RF stations were installed giving us some margin and allowing us to run with up to $2 \mathrm{RF}$ stations down. The RF voltage and bucket area during the current MI ramp are shown in Figure 2.66.

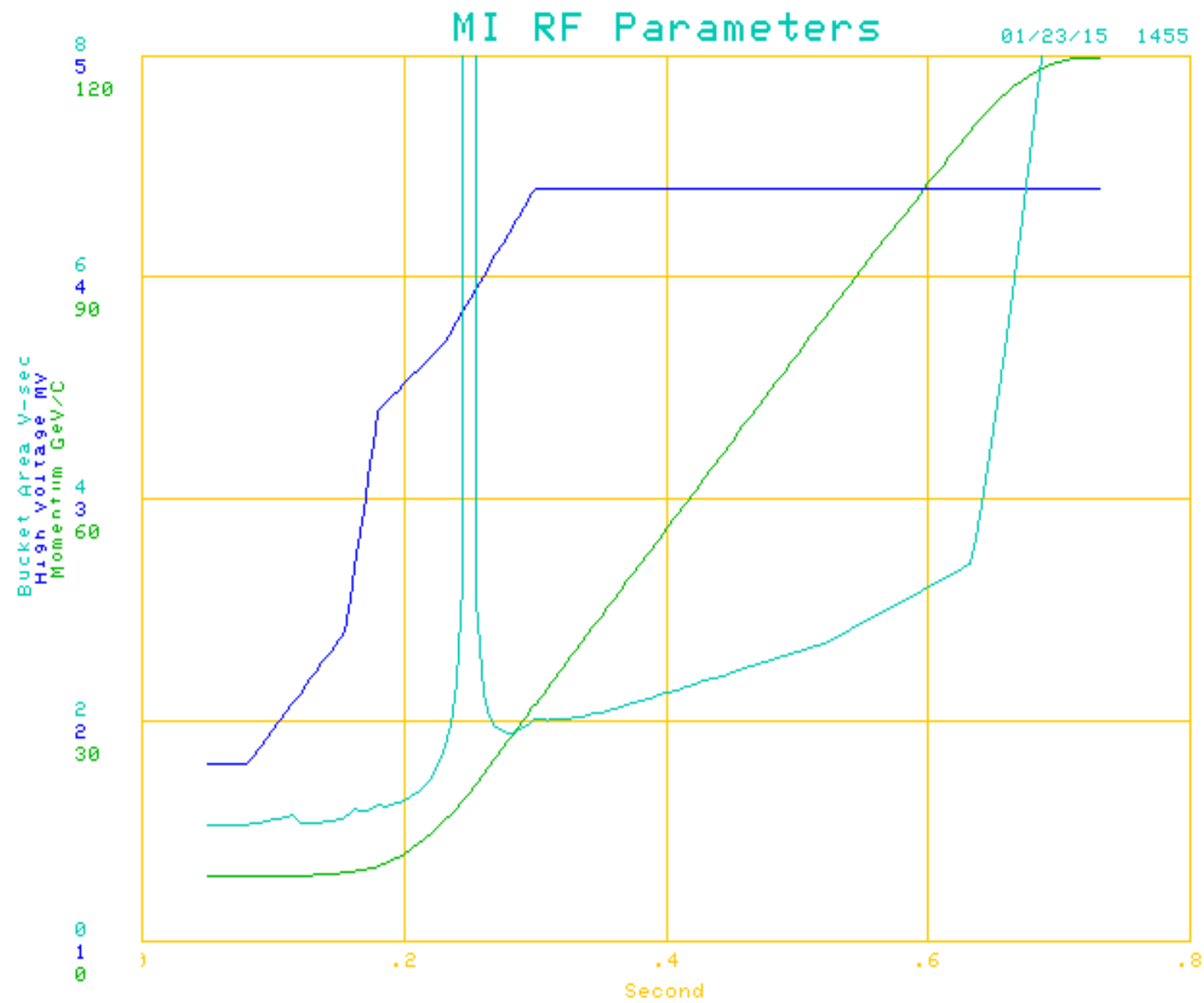

Figure 2.66: RF Voltage and bucket area during the MI acceleration with present cycle.

The MI ramp can be configured for different momenta. Figure 2.67 presents the MI cycle time vs beam momentum for PIP-II operation. The cycle times have been rounded up to the nearest 1/20 s. Based on the cycle times the calculated MI beam power vs beam momenta is shown in Figure 2.68 . 


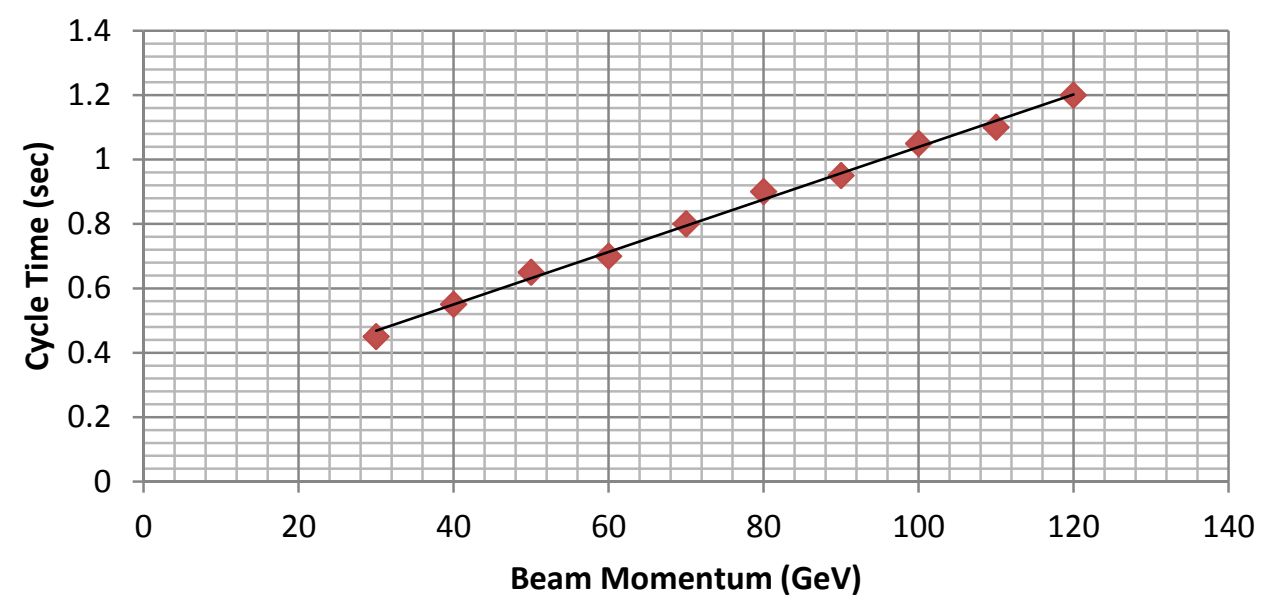

Figure 2.67: MI cycle time vs beam momenta.

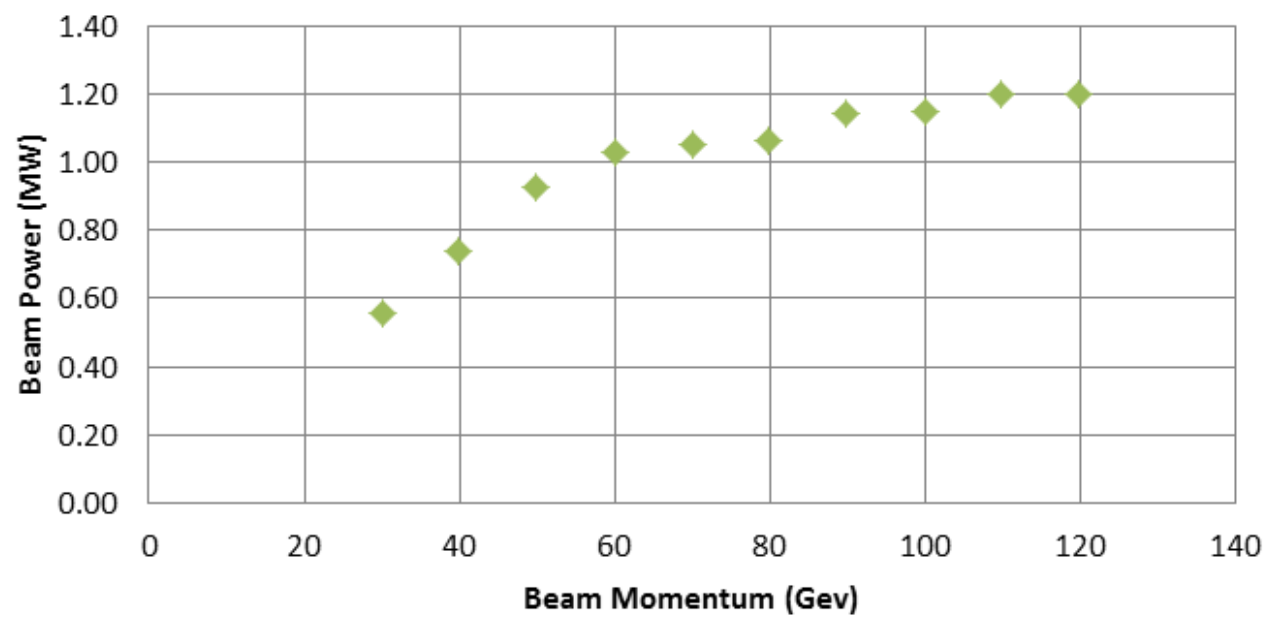

Figure 2.68: MI beam power vs beam momenta.

\subsubsection{Transition Crossing}

MI transition crossing is dominated by non-linear effects because of the large longitudinal emittance. Since the Booster will be upgraded to run at $20 \mathrm{~Hz}$ instead of $15 \mathrm{~Hz}$ the frequency separation for slip stacking will be increased to $1680 \mathrm{~Hz}$ from present $1260 \mathrm{~Hz}$ resulting in larger longitudinal phase space area at injection and, consequently, larger longitudinal emittance after recapture in the MI. To reduce the effects of the transition crossing a first order gamma-t jump has been considered.

For MI the non-adiabatic time $T_{c}$ which represents the time during which the longitudinal motion of the synchronous particle is not well represented by a slowly varying Hamiltonian, is around $1.5 \mathrm{msec}$. The nonlinear time $T_{n l}$ which parameterizes the Johnsen effect [56, p. 285], in which particles with different momenta cross the transition at different times, is $2.3 \mathrm{~ms}$. The nominal bipolar jump illustrated in Figure 2.69 maintains a clearance of

$$
\left|\gamma-\gamma_{t}\right| \geq 1 \sim 2 \gamma^{\prime} T_{n l}
$$


except for about $0.5 \mathrm{~ms}$. Transition is crossed at $d\left(\gamma-\gamma_{t}\right) / d t=4000 \mathrm{~s}^{-1}$ almost sixteen times faster than without the jump.

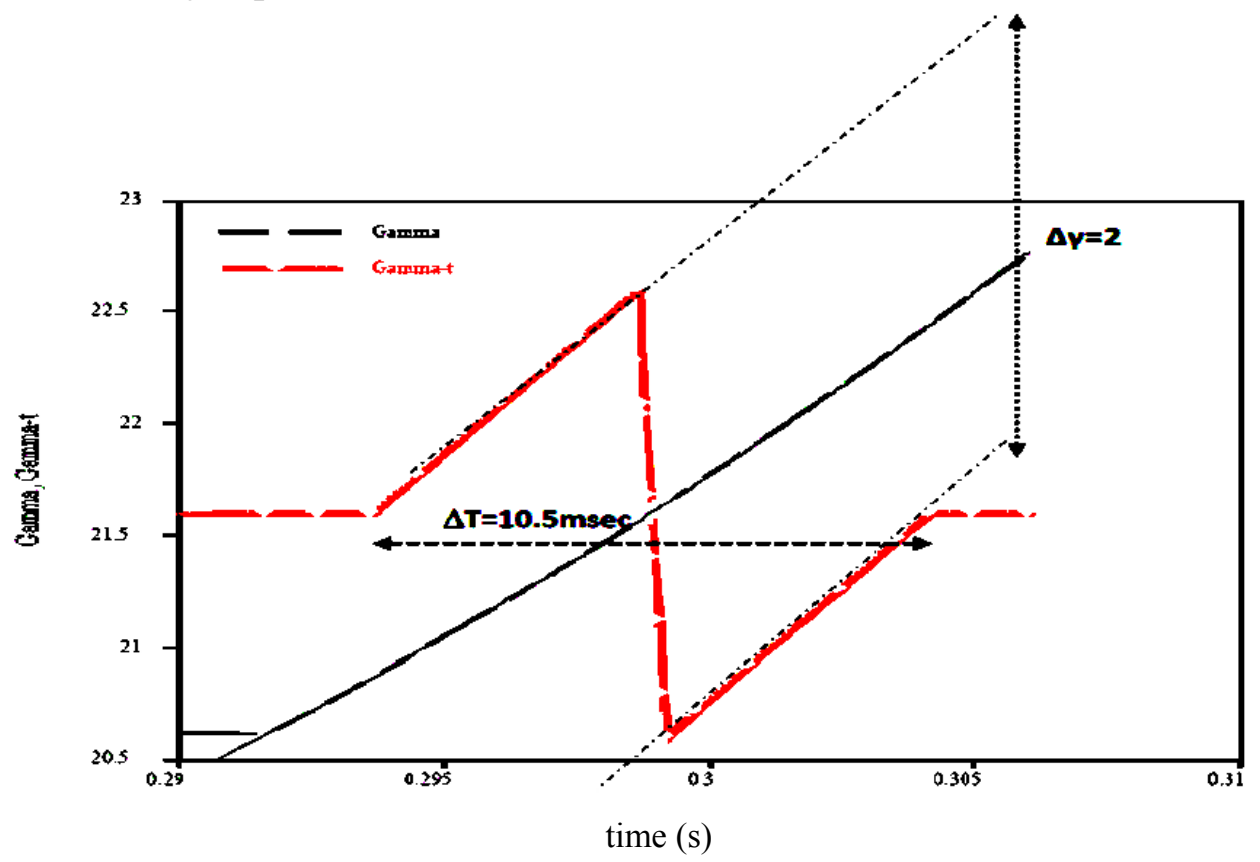

Figure 2.69: MI bipolar gamma-t jump. Transition is crossed 16 times faster with the transition jump than without.
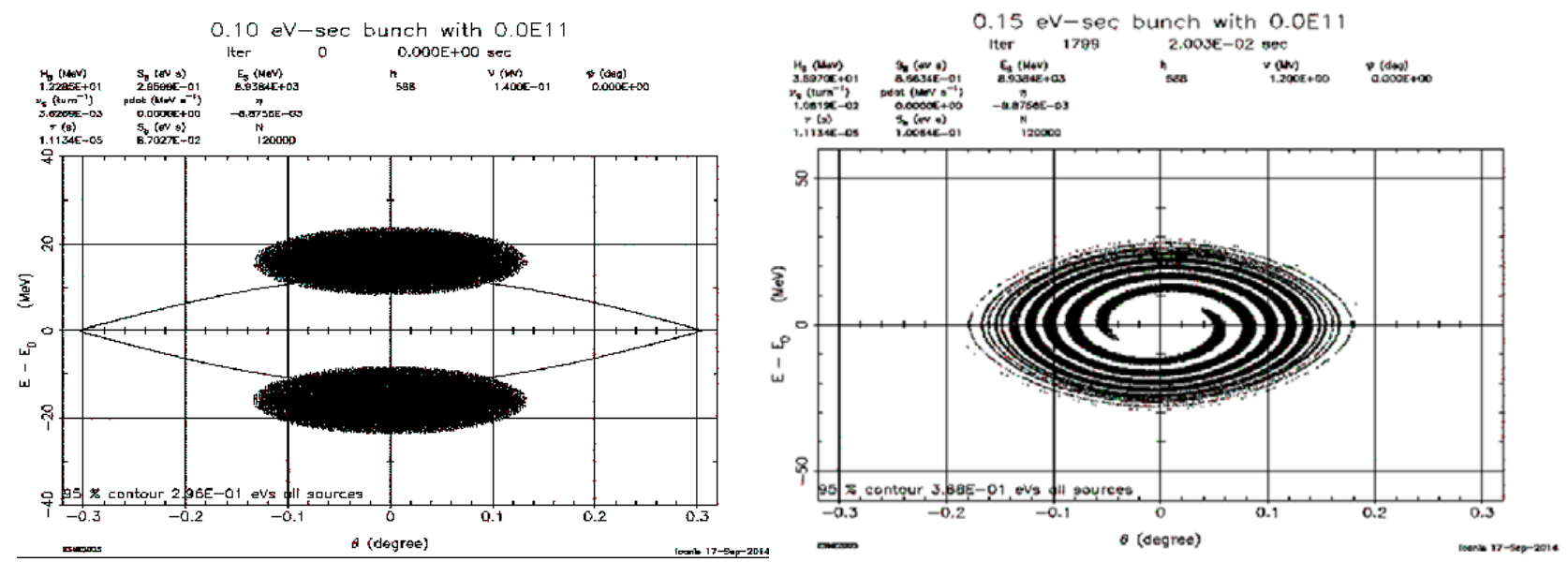

Figure 2.70: Initial condition for the ESME simulation. In the left picture two $0.1 \mathrm{eV}$-sec bunches captured in $145 \mathrm{kV}$ buckets separated by $1680 \mathrm{~Hz}$ are shown. In the right picture the resulting distribution after recapture with 1.2 MV in MI is shown.

A series of ESME simulations were performed. They accounted for the space charge impedance, $Z / n$ and the gamma-t jump. The initial conditions are shown in Figure 2.70. The final bunch distribution after recapture in MI has a bunch area of $0.5 \mathrm{eV}-\mathrm{sec}(100 \%)$. Figure 2.71 presents the pdot and the RF voltage curve used for the MI ramp in the ESME simulations.

The beam distributions after transition with and without gamma-t jump are shown in Figures 2.72 and 2.73. From Figure 2.72 it can be seen that without a gamma-t jump the low energy tail of the bunch that develops after transition is similar in the cases with zero charge and full charge 
indicating that it is non-linear effects and no space charge that is dominating the transition crossing in MI. The low energy tail exceeds the momentum aperture of MI leading in beam scraping and beam loss. With the full gamma-t jump the low energy tail is eliminated while with half the jump the beam is contained within the MI momentum aperture as presented in Figure 2.73.
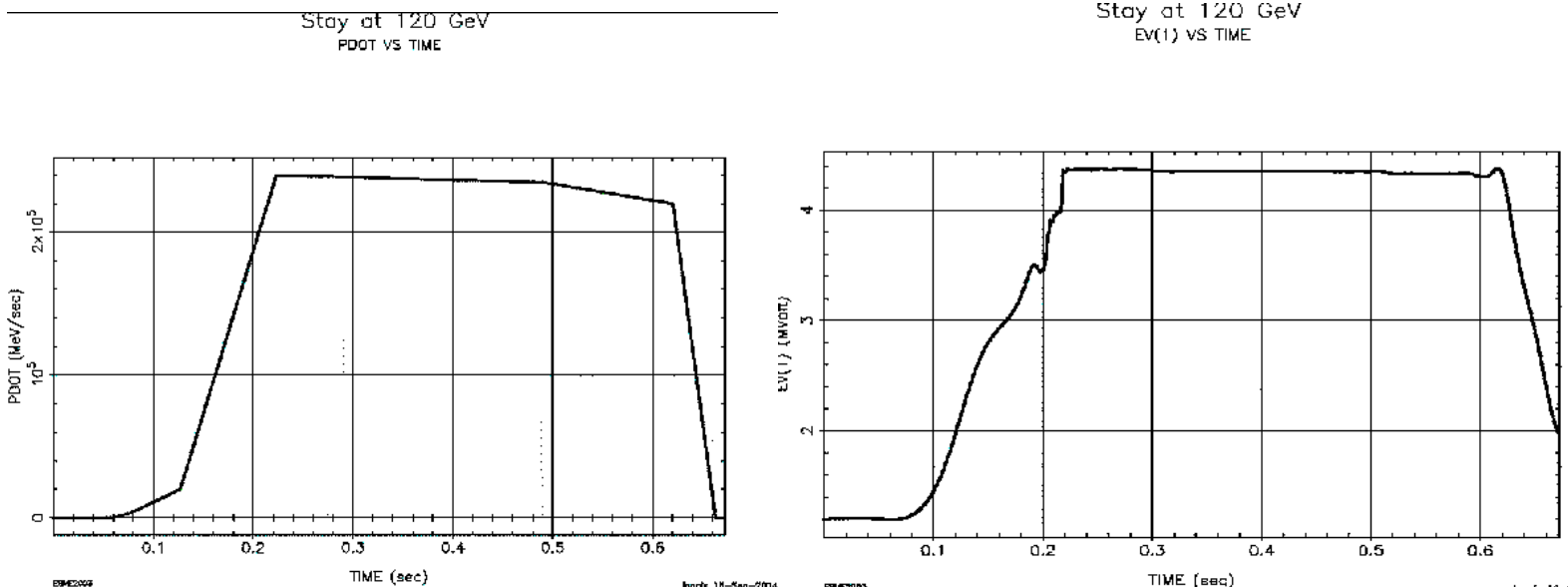

Figure 2.71: Pdot and RF voltage during the MI ramp used in ESME simulations.

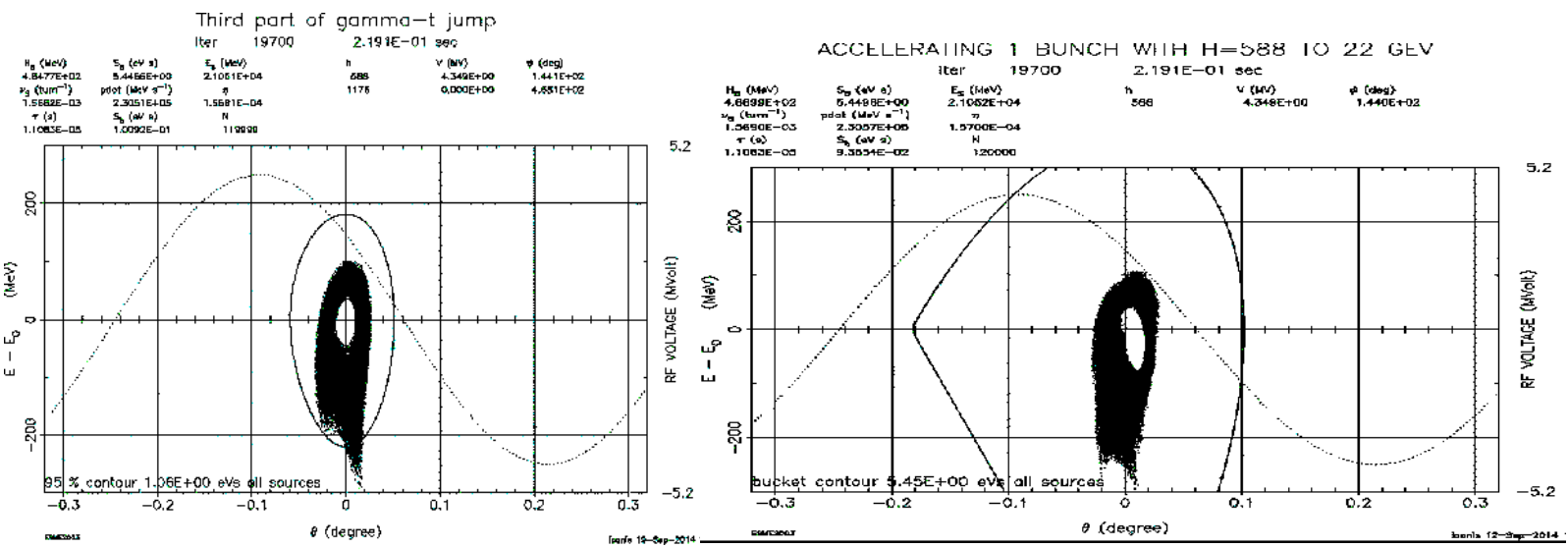

Figure 2.72: Phase space distribution after transition in MI with zero charge (left) and full charge (right).

\subsubsection{Beam Stability in the Recycler and MI}

The main beam parameters used for in the Recycler stability estimate are listed in Table 2.22. At injection $(\gamma=9.5)$ the space charge is strong transversely, i.e. the tune shift $\Delta Q_{s c} \approx 0.1$ is much larger than the synchrotron tune $Q_{s} \approx 0.0034$; the space charge is also important longitudinally, leading to the synchrotron tune depression of about $20 \%$ [51], and to a possibly of longitudinal instability similar to the "dancing bunches" [54]. The longitudinal instability can be significantly exacerbated by coupled-bunch interaction through high order modes (HOM) in the cavities, leading to the growth rate [55]:

$$
\begin{aligned}
\tau_{\|}^{-1} & =\frac{M N r_{0} \eta R_{s} \omega_{r} \rho_{\omega_{r}}^{2}}{\gamma C Q_{s} Z_{0}} ; \\
\rho_{\omega_{r}}^{2} & =\exp \left(-\omega_{r}^{2} \sigma_{\tau}^{2}\right) ; \quad Z_{0}=377 \Omega .
\end{aligned}
$$


Here $R_{s}$ is the shunt impedance, $\omega_{r}$ is the HOM frequency, and $\sigma_{\tau}$ is the rms bunch length in time units. For $R_{s}=35 \mathrm{k} \Omega, \omega_{r} /(2 \pi)=150 \mathrm{MHz}$, and $\sigma_{\tau}=1.9 \mathrm{~ns}$ this yields a rather high frequency suppression factor $\rho_{\omega_{r}}^{2}=0.03$, leading to $\tau_{\|}=30 \mathrm{~ms}, \tau_{\|}^{-1}=0.02 \omega_{s}$. A narrow-band damper could suppress such slow coupled-bunch motion if it will be required.
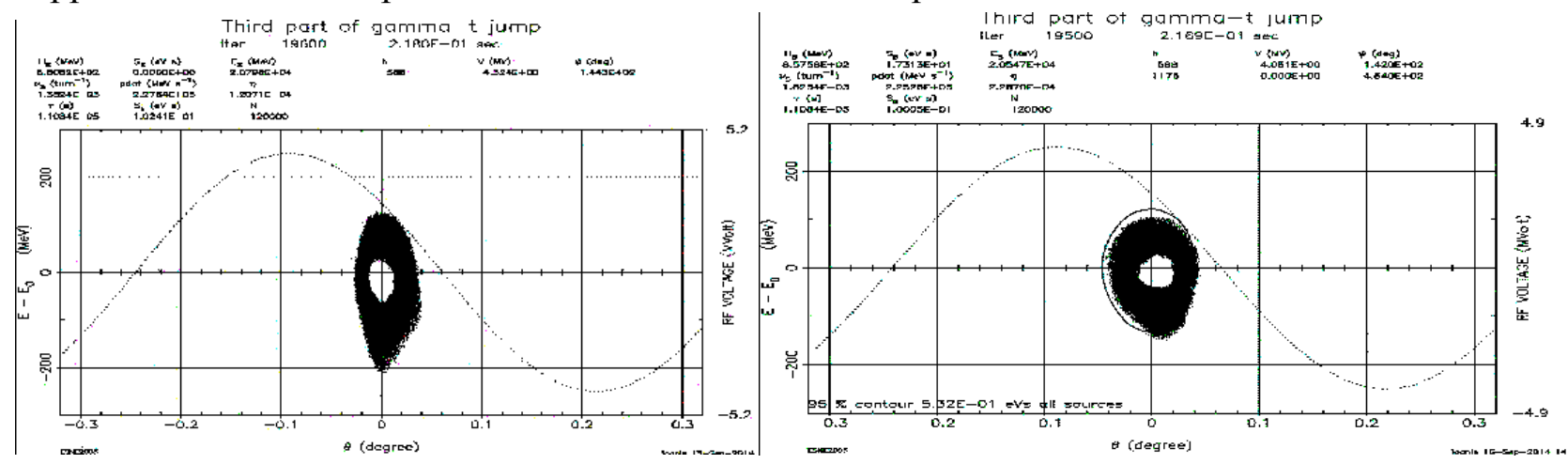

Figure 2.73: Phase space distribution after transition with full charge and two different sizes of the gamma-t jump. Half jump (left) and whole jump (right).

Table 2.22: Beam parameters in the Recycler used in beam stability estimates

\begin{tabular}{l|l|l}
\hline Bunch population, $N$ & $8.2 \cdot 10^{10}$ & \\
\hline Number of bunches & $81 \cdot 6 \cdot 2=972$ & \\
\hline Transverse emittance, norm. rms, $\varepsilon_{n \perp}$ & 2.5 & $\mathrm{~mm} \mathrm{mrad}$ \\
\hline Longitudinal emittance, rms, $\varepsilon_{\|}=\sigma_{\tau} \sigma_{E}$ & 3.6 & $\mathrm{meV} \mathrm{s}$ \\
\hline Maximal RF Voltage, $V$ & 0.125 & $\mathrm{MV}$ \\
\hline Transition gamma, $\gamma_{t}$ & 21.6 & \\
\hline \hline
\end{tabular}

The transverse single-bunch instability is described by a growth rate [49]

$$
\tau_{\perp \mathrm{SB}}^{-1}=F_{\mathrm{SB}}(\chi) \frac{N r_{0} \bar{W}_{\mathrm{B}} \bar{\beta}}{4 \pi \gamma}
$$

where $F_{\mathrm{SB}}(\chi) \leq 0.1$ is the chromaticity factor determined by the head-tail phase $\chi=\zeta \sigma_{s}, \bar{W}$ is a bunch-averaged wake function, $\bar{\beta}$ is the average beta-function. For $\chi \geq 1$, the chromaticity factor saturates at its maximum, $F_{\mathrm{SB}}(\chi) \simeq 0.1$. For the resistive wall case, with the half-gap $b$ and the conductivity $\sigma$, the average wake is estimated as

$$
\bar{W}_{\mathrm{SB}}=\frac{\pi^{2}}{12} \frac{2 C}{\pi b^{3} \sqrt{\sigma \sigma_{\tau}}}
$$

Altogether, this leads to single-bunch instability with a growth time $\tau_{\perp \mathrm{SB}}=20 \mathrm{~ms}, \tau_{\perp \mathrm{SB}}^{-1}=0.03 \omega_{\mathrm{s}}$.

The coupled-bunch transverse impedance due to wall resistivity is:

$$
Z_{\mathrm{CB}}=Z_{0} R \frac{\pi^{2}}{12} \frac{\delta}{b^{3}},
$$


where $\delta$ is the skin depth at the corresponding coupled-bunch frequency. The corresponding instability growth rate is:

$$
\tau_{\perp \mathrm{CB}}^{-1}=\left|I_{n}(\chi)\right|^{2} \frac{M N r_{0} \bar{\beta}}{\gamma T} \frac{Z_{\mathrm{CB}}}{Z_{0} R},
$$

where the coupled-bunch chromatic form-factor $I_{n}(\chi)$ is given by Eq. (2.13). For the parameters of Table 2.22, this yields the growth rate close to the synchrotron frequency, $\tau_{\perp \mathrm{CB}}^{-1}=1.2 \omega_{s}=1.8 \cdot 10^{3} \mathrm{~s}^{-1}$. Suppression of that fast instability most likely would require both the transverse damper and rather strong octupoles.

Currently, the Recycler performance is limited by the electron cloud instability. While beam scrubbing is gradually elevating the thresholds, a future need for the chamber surface coating is questionable.

\subsubsection{Electron Cloud Mitigation}

Electron cloud generation could be a possible instability source for the intensities in the Recycler and Main Injector.

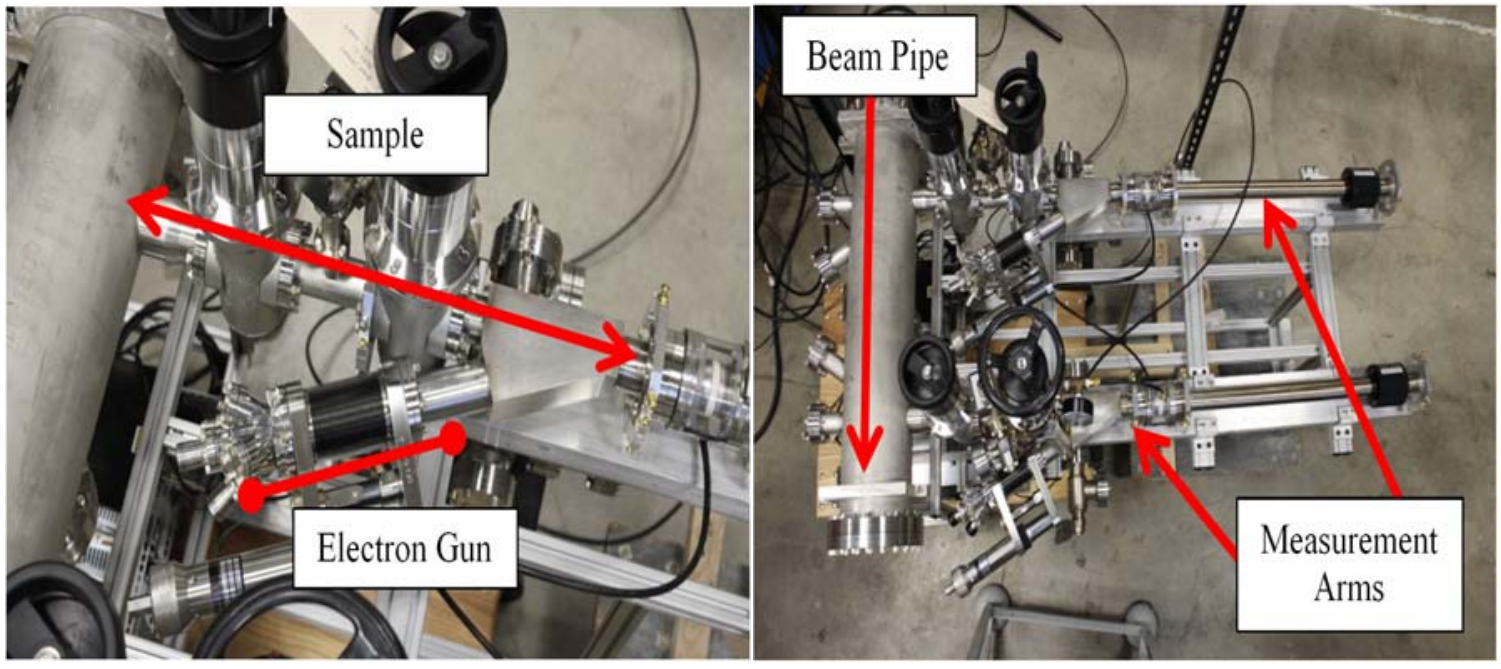

Figure 2.74: SEY Measuring Stand.

The best approach is to mitigate the generation of the cloud itself. There have been a series of measurements in the Main Injector, looking at secondary electron yield and cloud generation. A dedicated measurement setup now exists at MI-52, with newly developed RFA detectors. Both TiN and $\mathrm{C}$ coated beam pipes have been installed and measurements made. Both coatings show significant reductions in secondary electron generation when compared to an uncoated stainless steel pipe. VORPAL simulations are being benchmarked against these measurements. There is a plan to install a SEY (Secondary Emission Yield) stand in MI in order to measure the effect of scrubbing in situ for different kinds of stainless steel. A picture of the SEY measuring stand is shown in Figure 2.74.

Research continues into the coating process. The Main Injector beam pipe is captured in the dipole magnets, so coating needs to take place in situ. A coating (sputtering) facility is set up in E4R and has successfully coated with $\mathrm{TiN}$ a $6 \mathrm{~m}$ long piece of round MI pipe and measured the 
coating thickness. It will be used to coat test coupons for SEY measurements in MI. The experience from our coating facility will be used to estimate the effort required to in-situ coat the MI beam pipe with TiN. 


\section{DESIGN CONCEPTS OF MAJOR SUBSYSTEMS}

\subsection{SC Linac}

\subsubsection{Warm Frontend}

The PIP-II frontend consists of an ion source, Low Energy Beam Transport (LEBT), Radio Frequency Quadrupole (RFQ), and Medium Energy Beam Transport (MEBT). The $\mathrm{H}^{-}$beam originates from a nominally $5 \mathrm{~mA}$ (nominal, $10 \mathrm{~mA}$ peak) DC ion source and is transported through the LEBT to a CW normal-conducting RFQ, where it is bunched and accelerated to 2.1 $\mathrm{MeV}$. In the MEBT a bunch-by-bunch chopper provides the required bunch patterns, removing 60$80 \%$ of bunches according to a pre-programmed timeline. To foresee possible upgrades, all elements of the frontend are designed for beam currents of up to $10 \mathrm{~mA}$. The beam energy of 2.1 $\mathrm{MeV}$ is chosen because it is below the neutron production threshold for most materials.

\subsubsection{Ion Source}

The ion source assembly is a $\mathrm{DC}, \mathrm{H}^{-}$source capable of delivering up to $10 \mathrm{~mA}$ of beam current at $30 \mathrm{keV}$ to the LEBT. The ion source specifications are listed in Ref. [57]. The present scenario assumes the volume cusp ion source presently commercially available from D-Pace Inc. ([58], Figure 3.1). This source is capable of delivering up to $15 \mathrm{~mA}$ with a satisfactory transverse emittance of $<0.2 \mu \mathrm{m}$ (rms, normalized), but its life time is relatively short. The main reason is the source filament, which needs to be replaced every 300 hours. To improve the beam uptime, two ion sources are planned to be installed (see Figure 2.2). Each source can be removed for repairs, installed back, and conditioned without interrupting the operation of the other source.

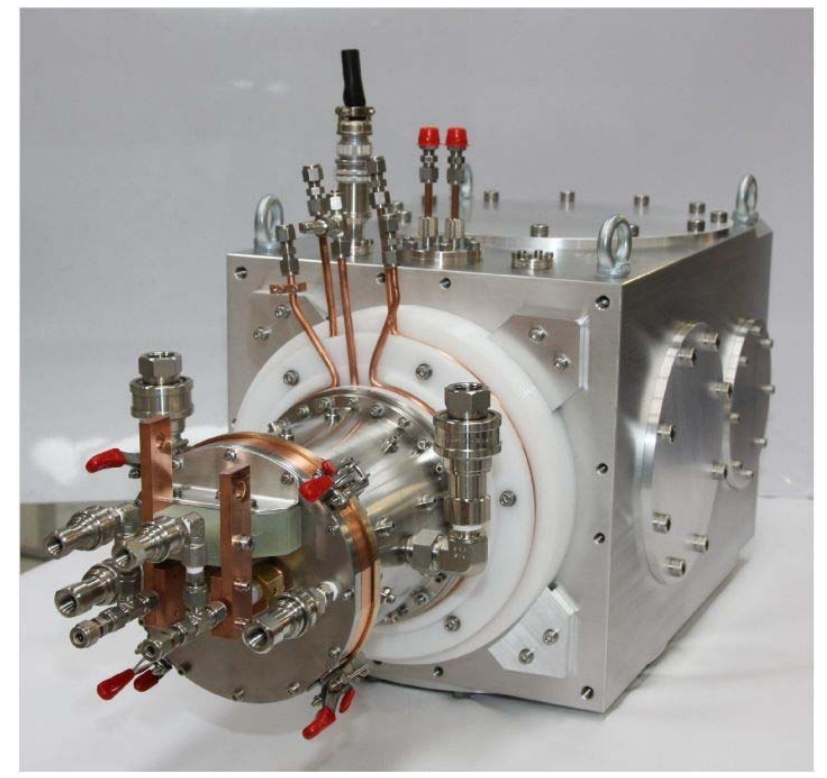

Figure 3.1: Photograph of the D-Pace ion source (foreground) with the vacuum chamber.

\subsubsection{LEBT - Low Energy Beam Transport}

The LEBT transports the beam from the exit of the ion source to the RFQ entrance and matches the optical functions to those of the RFQ. In addition, the LEBT forms a low-duty factor beam 
during commissioning and tuning of the downstream beam line and interrupts the beam as part of the machine protection system.

The functional requirement specifications are listed in Ref. [15], and an LEBT layout is shown in Figure 2.2. The LEBT includes 3 solenoids (for each leg), a dipole magnet to switch ion sources, a chopper assembly (a kicker followed by a beam absorber), and diagnostics to characterize and to tune the beam. The length of the beam line, $\sim 2 \mathrm{~m}$, insures that the gas migration from the ion source to the RFQ is tolerable.

The beam diagnostics include an emittance scanner (see specifications in [59]) at the exit of each ion source, a DCCT, and a toroid. In addition, fixed electrically-insulated diaphragms are installed inside the solenoids. Moving the beam by dipole correctors, built into each solenoid, and measuring the current at the downstream diaphragm enables estimating the beam size and its position. The scraper in front of the RFQ is a movable, electrically insulated, water-cooled plate that can be moved into three positions: completely removed, partially inserted so that a round opening in the plate is concentric with the RFQ entrance diaphragm, and fully inserted to completely intercept the beam. The scraper serves several purposes: the size of the opening is chosen to scrape the halo particles that otherwise would be lost in the RFQ or MEBT; the variation of beam current intercepted by the scraper while moving the beam across the opening with upstream dipole correctors gives information about the beam position and core size; and the fully inserted scraper works as a beam stop and an auxiliary beam current monitor.

Fast machine protection and pulsed beam operation are achieved via the chopper assembly, which comprises an electrostatic kicker followed by an absorber. In some scenarios, it can also be used as a pre-chopper to assist the MEBT chopping system. The primary machine protection mechanism is to disable the beam from the ion source by turning off its extraction and bias voltages, with the LEBT chopper serving as a fast beam switch during ion source turn-off.

For the PIP-II LEBT the optics design incorporates two regions (see Figure 2.3). First, the beam is nearly fully neutralized from the exit of the ion source to immediately upstream of the kicker. Further downstream, the beam can be either neutralized or un-neutralized. In the un-neutralized mode, the secondary ions created in that area are removed by a constant electric field on the kicker plates, and the ions from upstream are stopped by a positive voltage on the insulated diaphragm \#2. In the neutralized mode, the kicker plates as well as the insulated diaphragm \#2 have normally ground potential, while the insulated diaphragm \#3 or the scraper is biased positively to prevent the ion escape longitudinally. The LEBT scheme is flexible enough to accommodate both versions by adjusting potentials and solenoid currents (see Figure 2.4) for simulations of a fully neutralized transport case). The transport with an un-neutralized section is beneficial for decreasing the transition effects of the kicker pulse but results in an emittance increase. The relative benefits of each scenario can be clarified during PXIE experiments.

\subsubsection{RFQ - Radio-Frequency Quadrupole Accelerator}

The $162.5 \mathrm{MHz}$ CW RFQ will accelerate an $\mathrm{H}^{-}$ion beam with currents of up to $10 \mathrm{~mA}$ from 30 $\mathrm{keV}$ to $2.1 \mathrm{MeV}$ (see Ref. [60] for specifications). Presently the PIP-II RFQ is assumed to be identical to that being developed for PXIE by LBNL [61]. This 4.45-m long, four-vane structure consists of four longitudinal modules and uses a $60 \mathrm{kV}$ vane-to-vane voltage. Most of the RF input power is dissipated in the cavity walls resulting in $\sim 12 \%$ beam loading at $5 \mathrm{~mA}$. A series of 32 water-cooled pi-mode rods provides quadrupole mode stabilization, and a set of 80 evenly spaced fixed slug tuners is used for the final frequency adjustment and local field perturbation corrections. The design incorporates selected portions of the technology validated by the Spallation Neutron 
Source (SNS) Front End RFQ [62] designed and constructed at LBNL. An overall view of the full four-module RFQ is shown in Figure 3.2.

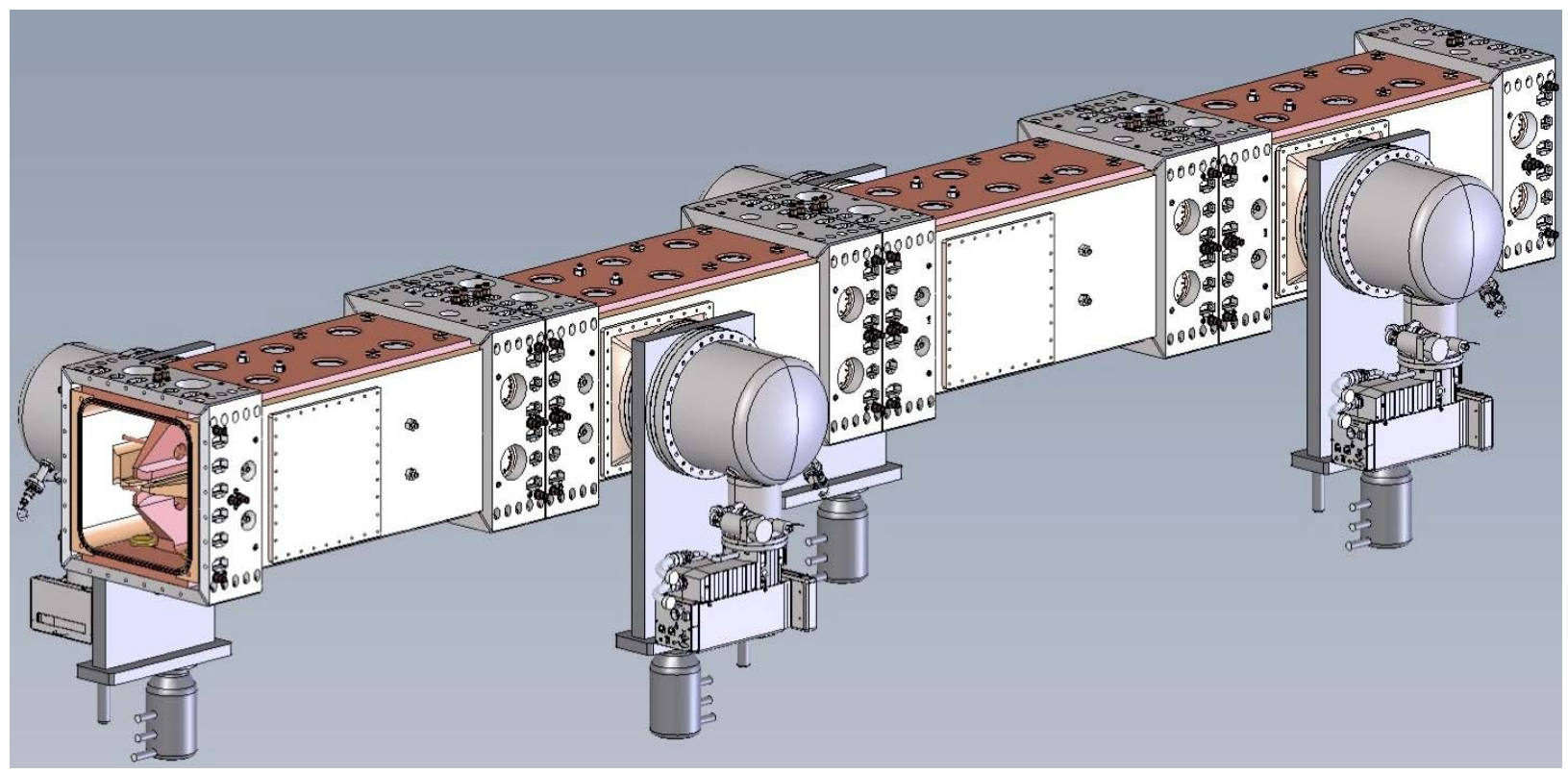

Figure 3.2: CAD model of the full four-module RFQ.

The beam dynamics design of the RFQ provides over $96 \%$ transmission for beam current from 1 to $15 \mathrm{~mA}$ (see Figure 2.7). At $5 \mathrm{~mA}$ nominal current, $99.8 \%$ beam capture is achieved with transverse and longitudinal emittance (rms, norm) of $0.15 \pi-\mathrm{mm}-\mathrm{mrad}$ and $0.64 \mathrm{keV}$-nsec, respectively. The RF design studies [63] include mode stabilization, field flatness, radial matching, and entrance and exit terminations. Table 3.1 summarizes the RF design results.

Table 3.1: Main parameters of the PIP-II RFQ electromagnetic design

\begin{tabular}{l|l}
\hline \hline Parameter & Value \\
\hline Frequency, $\mathrm{MHz}$ & 162.493 \\
\hline Frequency of dipole mode, $\mathrm{MHz}$ & 181.99 \\
\hline Q factor & 14660 \\
\hline Max power density, $\mathrm{W} / \mathrm{cm}^{2}$ & 7.9 \\
\hline Total power loss, $\mathrm{kW}$ & 74.6 \\
\hline Beam power $@ 5 \mathrm{~mA}, \mathrm{~kW}$ & 10.5 \\
\hline Total RF power from source, $\mathrm{kW}$ & 150 \\
\hline \hline
\end{tabular}

A series of RF and thermal finite-element models of the RFQ have been developed using ANSYS $^{\circledR}$. An example of the temperature contour plots for the cavity body and vane cutback region is shown in Figure 3.3. From the RF analysis, the average linear power density was determined to be $137 \mathrm{~W} / \mathrm{cm}$ with a peak heat flux on the cavity wall of only $0.7 \mathrm{~W} / \mathrm{cm}^{2}$. With $30^{\circ} \mathrm{C}$ water in the vane and wall cooling passages, the resulting temperature profile in the cavity body ranges between 32 and $37^{\circ} \mathrm{C}$ at full RF gradient. 
Additional modeling has been carried out. It includes stress and displacement analyses, thermal analyses of the tuners, pi-mode rods and vane cutbacks, and prediction of the frequency shift of the RFQ cavity due to thermal loading and changes in the cooling water temperature.

The RFQ cooling scheme will use differential water temperature control in the vane and wall passages. This technique provides active tuning of the RFQ by holding the wall water temperature constant and adjusting the vane water temperature up and down. The frequency of the RFQ can be shifted by $-16.7 \mathrm{kHz}$ for every $1{ }^{\circ} \mathrm{C}$ rise in the vane cooling water temperature. For uniform water temperature control, the shift would only be $-2.8 \mathrm{kHz} /{ }^{\circ} \mathrm{C}$.
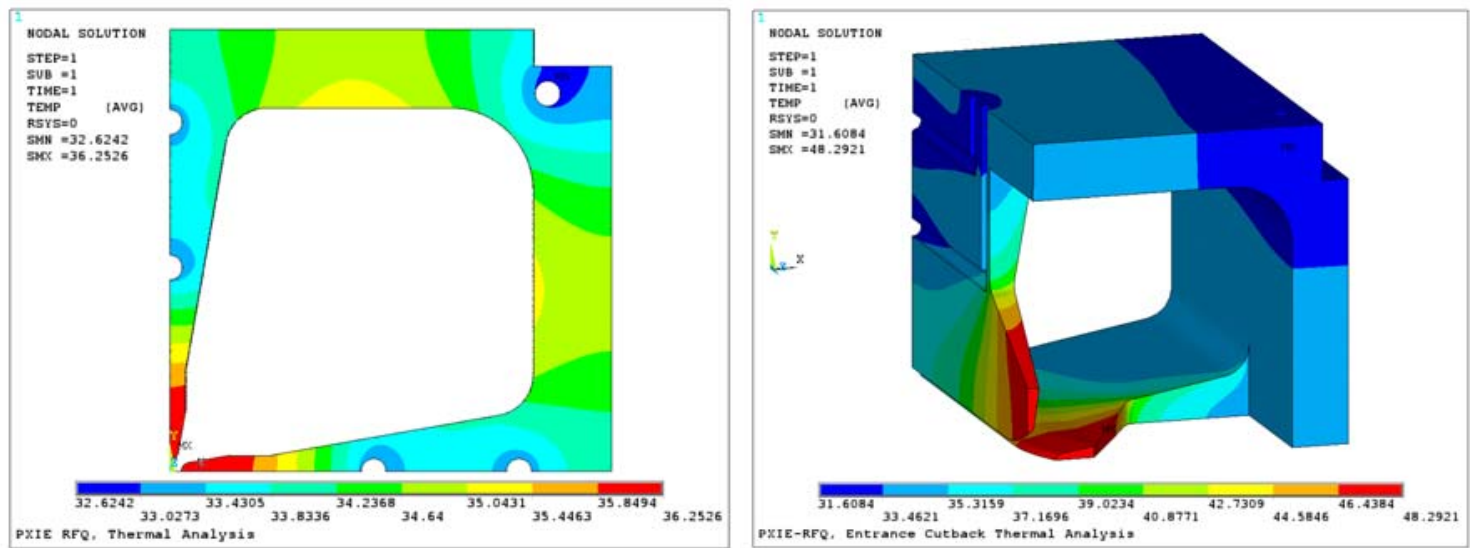

Figure 3.3: Temperature distribution in one RFQ quadrant body (left) and cut-back (right). The color scheme (degrees $\mathrm{C}$ ) is at the bottom of each plot.

\subsubsection{MEBT - Medium Energy Beam Transport}

The MEBT layout is given in Figure 2.11. The MEBT generally provides beam transport and focusing (both transverse and longitudinal), beam chopping, chopped beam absorption, vacuum pumping, and diagnostics. Transverse focusing is provided primarily by equidistantly placed quadrupole triplets; the only exception is two doublets at the RFQ exit. Each triplet or doublet is followed by a pair of dipole correctors. The specifications for the quadrupoles and correctors are listed in [64]. The spaces between neighboring triplets or doublets are referred to as MEBT sections, with each section providing a particular service. The section separation in the regular part of the MEBT is $1140 \mathrm{~mm}$, which leaves a 650-mm long (flange-to-flange) space for various equipment ( $350 \mathrm{~mm}$ in the section between doublets labeled $\# 0$ ).

The undesired beam bunches will be removed in the MEBT by a chopping system, represented in Figure 2.11 by red boxes. The system consists of two identical $50 \mathrm{~cm}$ long kickers separated by a $180^{\circ}$ transverse phase advance and an absorber ( $90^{\circ}$ from the last kicker). In the broadband, travelling-wave kicker, the transverse electric field propagates with the phase velocity equal to the speed of $\mathrm{H}^{-}$ions $(\sim 20 \mathrm{~mm} / \mathrm{ns}, \beta=0.0668)$ so that the ion vertical velocity changes sufficiently to displace the ion bunches, designated to be removed, to the absorber. The separation between the kicker plates is $16 \mathrm{~mm}$. The aperture is limited by protection plates on both sides of the kicker to $13 \mathrm{~mm}$ so that in the case of a mismatched transport, the intercepted bean current on these protection plates would trigger the beam turn-off. Detailed specifications for the kicker can be found in Ref. [65]. The simulated transverse beam envelopes in the MEBT for both passing and chopped bunches are presented in Figure 2.12.

Presently two versions of the kicker, which differ by the structure's impedance, are being 
investigated [23]. In the $50 \mathrm{Ohm}$ version, the kicker plates are connected in vacuum by cable delay lines (Figure 3.4 purple loops). Compared to the helical structure discussed below, this arrangement considerably reduces coupling between neighboring turns and consequently dispersion. Each kicker is driven by two commercially available linear amplifiers. Signal distortion caused by the imperfections of the amplifier characteristics, cabling, and dispersion in the structure are corrected by the corresponding pre-distortion of the amplifier's input signal.

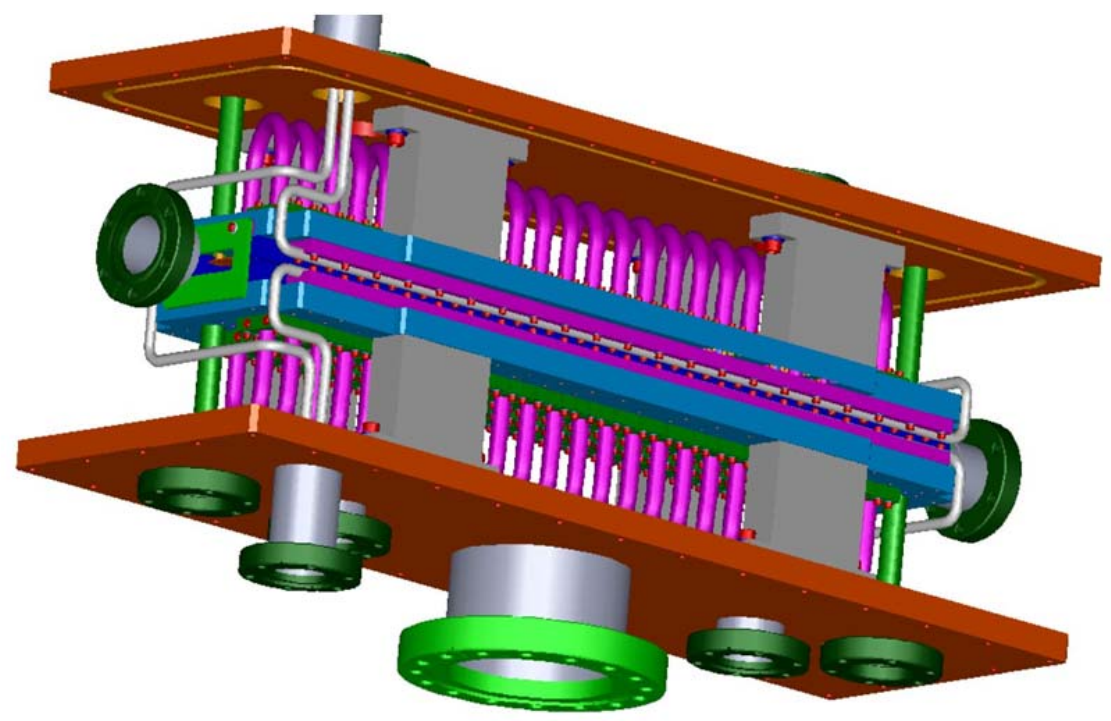

Figure 3.4: 3-D model of the 50-Ohm kicker structure (side walls of the vacuum box are removed for presentation purpose).

The 200-Ohm structure comprises two helical windings around grounded cylinders with plates attached to windings (Figure 3.5). In this scheme, the kicker driver is a fast switch being developed in-house. In addition, this scheme requires custom made feedthroughs, transmission lines, and current loads. While the $50 \mathrm{Ohm}$ version has many elements commercially available and the design of its structure is more mature, the $200 \mathrm{Ohm}$ is potentially less costly and has a lower power loss in the structure. The selection of the kicker technology for PIP-II will be based on beam tests at the PXIE.
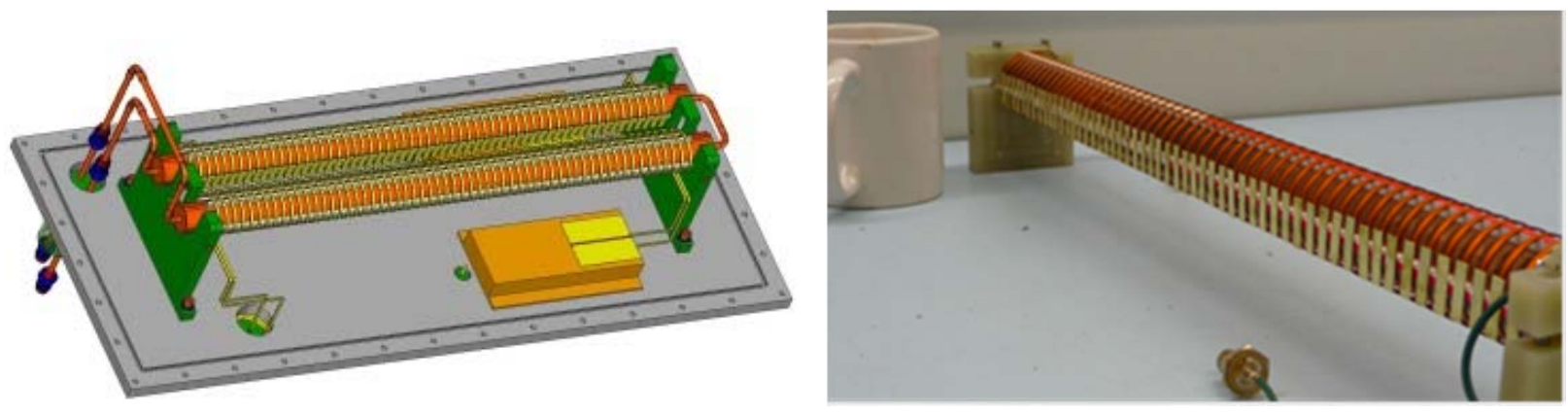

Figure 3.5: Conceptual design (left) and photograph (right) of a single-helix model of the $200 \mathrm{Ohm}$ dual-helix kicker. 
The undesired bunches are directed to an absorber that is displaced vertically from the beam trajectory. To accommodate the entire beam that the upstream system is capable to deliver, the absorber is being designed for the maximum beam power of $21 \mathrm{~kW}$ which corresponds to a $10-\mathrm{mA}$ beam completely diverted to the absorber (see specifications in Ref. [66]). The power density in the beam with a $\sim 2 \mathrm{~mm}$ rms radius exceeds by an order of magnitude what is technically possible to absorb without melting the surface. To decrease the surface power density, the absorber is positioned at a small angle, $29 \mathrm{mrad}$, with respect to the beam (Figure 3.6).
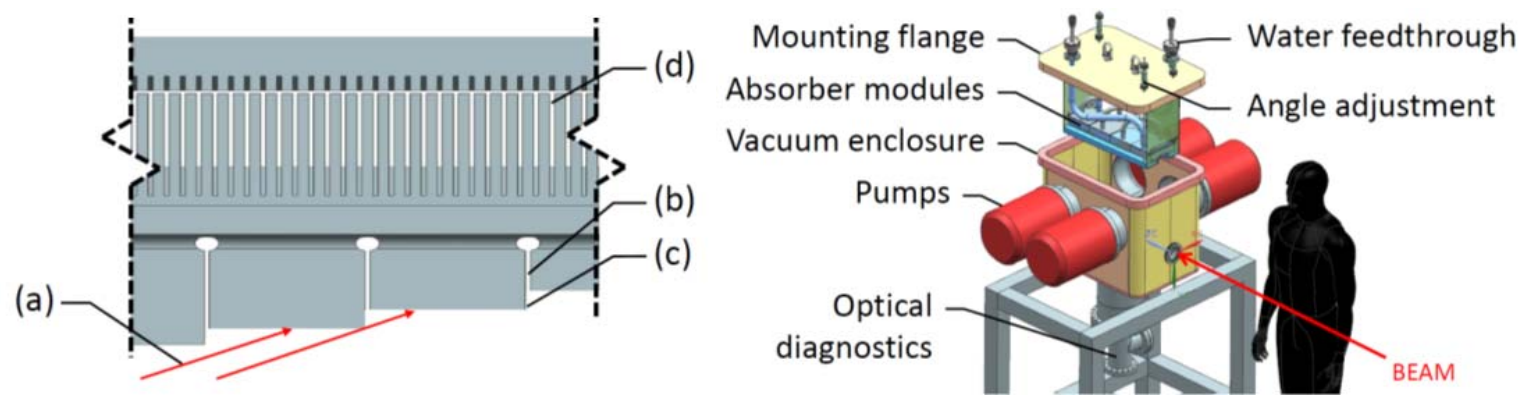

Figure 3.6: A conceptual design of the MEBT absorber. Left: a side-view of the absorber showing (a) beam incident on surface, (b) axial stress relief slits, (c) shadowing step increment (magnitude exaggerated), (d) $300 \mu \mathrm{m}$ wide by $1 \mathrm{~mm}$ pitch water cooling channels. The horizontal scale is exaggerated. Right: an exploded view.

Challenges in the absorber design include maintaining vacuum quality, managing surface effects (sputtering and blistering), containing secondary particles, accommodating radiation effects, spreading energy deposition, and the survival at high temperatures with temperature-induced mechanical stresses. Presently the design choice is an absorber with an absorbing surface that is composed of multiple pieces made from the molybdenum alloy TZM and pressed against a watercooled aluminum block [67].

To keep the beam properly bunched and to match the longitudinal phase space to the first superconducting cryomodule, the MEBT includes 3 identical (room temperature) bunching cavities $[68,69]$. Each cavity is a quarter-wave $162.5 \mathrm{MHz}$ resonator with the nominal accelerating voltage of $70 \mathrm{kV}$ (at $\beta=0.0668$ ). A conceptual view of the cavity being designed for PXIE and some results of its simulations are shown in Figure 3.7.
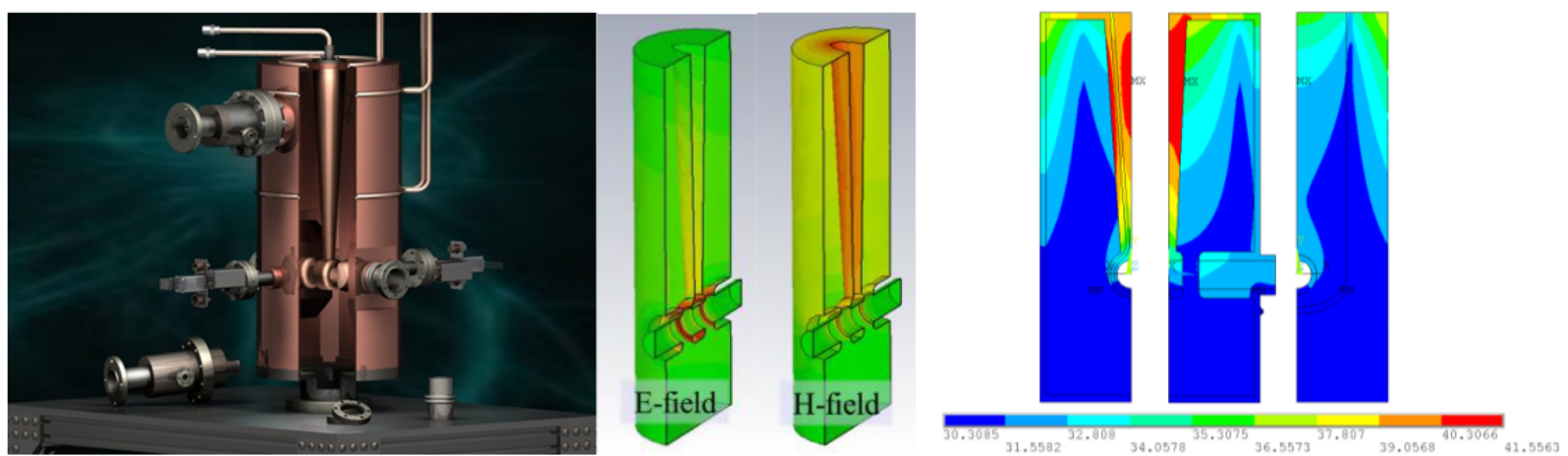

Figure 3.7: A conceptual design of the MEBT bunching cavity (left), simulated surface field distributions (center), and the temperature distribution (right) (from Ref. i). 
Each of the sections \#0, 1, 6, and 7 in Figure 2.11 contains a set of 4 scrapers. Each of these 16 scrapers is envisioned as an electrically insulated, $100 \mathrm{~W}$-rated plate precisely movable across the half-aperture [70]. The scrapers will be used for several purposes: for beam halo measurements and removal, protection of downstream equipment from a beam loss caused by beam envelope and trajectory mismatches, as an auxiliary beam density distribution diagnostics in the pulse mode, and formation of a pencil $\mathrm{H}^{-}$beam for measurements downstream (in pulsed mode). The scraper sets in the upstream and downstream pairs are separated by $\sim 90^{\circ}$ of betatron phase advance to insure an effective removal of particles with large transverse actions.

The vacuum requirements for the MEBT are determined by the electron detachment in $\mathrm{H}^{-}$beam and by the necessity to have a low gas flow into the HWR cryomodule. Obviously, the electron detachment results in a loss of $\mathrm{H}^{-}$beam intensity. An additional restrictive effect is creation of a flux of neutral hydrogen atoms that may reach the SRF cavities.

For the conceptual design of the vacuum system it is convenient to set a reasonable limit for the integral of the pressure over the distance along the MEBT axis. The selected value of $1 \times 10^{-6}$ Torr $\cdot \mathrm{m}$ corresponds to a relative loss of $\sim 10^{-4}$ and to an additional $\sim 0.1 \mathrm{~W}$ heat load to the SRF by neutral atoms.

Gas flow from the room-temperature MEBT to the $2 \mathrm{~K}$ HWR cryomodule can cause gas deposition on the cryogenic surfaces, which negatively affects the cavity performance. To limit this effect to safe levels, the vacuum pressure upstream of the HWR cryomodule is specified to be below $1 \times 10^{-9}$ Torr (hydrogen). To ensure this level, with a significant gas load coming from the absorber, $\sim 1 \mathrm{mTorr} \cdot 1 / \mathrm{s}$, the absorber section is followed by a differential pumping section and is separated from the SRF by two additional sections.

Most of the diagnostics are listed in Figure 2.11. Their arrangement will allow measuring the properties of the beam coming out of the RFQ. In addition, each triplet and doublet has a BPM inserted between quadrupoles. The BPMs will measure the transverse beam position and the bunch RF phase [71]. 


\subsubsection{Superconducting Accelerating Structures}

The parameters and requirements associated with all of the accelerating structures and cryomodules within the linac have been summarized in Tables 2.3, 2.4 and 2.5. This section describes design concepts for the cavity types required in the linac, and the associated cryomodules.

\subsubsection{Half-Way Resonator (HWR) Cryomodule}

The initial proposal included $325 \mathrm{MHz}$ Single Spoke Cavities of type 0 (SSR0), to accelerate the $\mathrm{H}^{-}$beam from 2.1 to $10 \mathrm{MeV}$. To maintain high beam quality, an adiabatic increase of the accelerating gradient in the SSR0 cavities was necessary, and satisfying the adiabaticity condition required 3 cryomodules comprising 24 SSR0 cavities. After careful consideration, a design based on 162.5-MHz Half-Wave Resonator (HWR) cavities was selected instead. This design has several substantial advantages if compared to the $325 \mathrm{MHz}$ SSR0 option:

- Only $8 \mathrm{HWRs}$ are required to accelerate the beam to $\sim 10 \mathrm{MeV}$ while maintaining high beam quality.

- Reduced RF defocusing due to both the lower frequency and the lower synchronous phase angle results in a much faster energy gain without emittance growth.

- It opens the possibility to use $162.5 \mathrm{MHz}$ rebunchers in the MEBT to allow for longer drift spaces for the fast beam choppers.

- Significant cost reduction due to the reduced component count.

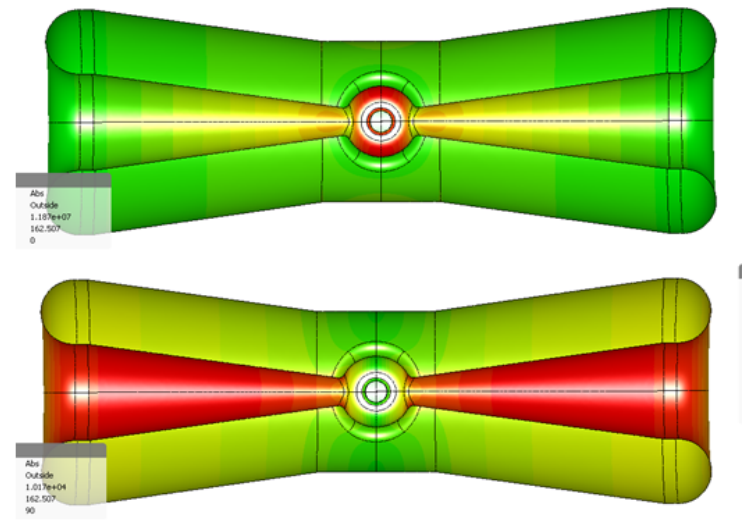

Figure 3.8: Left - Half-wave resonator model in Microwave Studio (MWS). The picture shows electric (top) and magnetic field (bottom) distributions on the surface. Red is high intensity and green is zero.

The beam dynamics optimization determines that a cavity beta of $\beta_{\mathrm{OPT}}=0.112$ is optimal. The cavity design is based on recent advances in SRF technology for TEM-class structures being developed at ANL. Highly optimized EM parameters which maximize the real-estate gradient while maintaining low dynamic cryogenic loads and peak surface fields were achieved using a conical shape for both the inner and outer conductors. A "donut" shaped drift tube in the center conductor (see Figure 3.8) has been developed to minimize the undesirable quadrupole component of the electric field as is shown in Figure 2.28. Utilization of the HWR requires two major subsystems: a $10 \mathrm{~kW}$ RF coupler and a slow tuner. A capacitive adjustable $10 \mathrm{~kW}$ RF coupler prototype has been designed, constructed, and successfully tested in 2014. It will provide RF power through the port which is perpendicular to the beam axis in the center of the cavity (Figure 
3.9). A pneumatically actuated mechanical slow tuner which compresses the cavity along the beam axis is located outside of the helium vessel and will be attached to the SS beam port flanges shown in Figure 3.9. A fast tuner is not required for $\mathrm{CW}$ operation anticipated for the HWR. The power margin (see Table 2.11) was chosen to be sufficient to suppress microphonics (mainly related to helium pressure fluctuations) without fast tuner. The required cavity bandwidth (loaded) is $60 \mathrm{~Hz}$. The main parameters of the HWR are shown in Tables $2.3-2.5$.

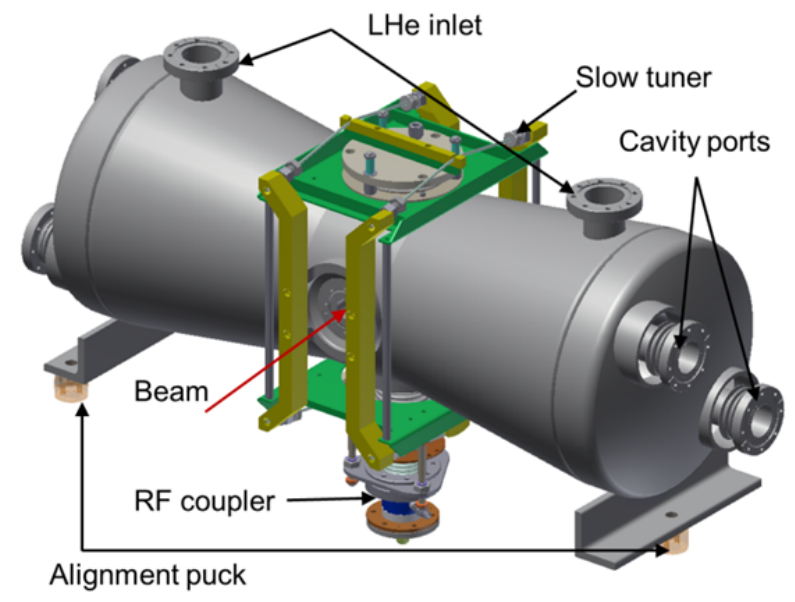

Figure 3.9: HWR cavity 3D model in INVENTOR

Extensive finite element analysis of the cavity included simulations to evaluate the integrity of the cavity per the Fermilab ES\&H manual. The simulations include protection against plastic collapse, local failure, buckling, ratcheting and fatigue failure to ensure that the operating loads are below the maximum allowable limits. The maximum structural load is determined by the pressure set by the operation of the cryogenic system and the safety pressure relief valve. The evaluation was performed for 30 psig at room temperature and 60 psig at $2 \mathrm{~K}^{0}$ in the helium space of the cavity in compliance with the Fermilab requirements. In general, the over pressure condition could occur during the initial cryogenic cooling with the cavity structure at or near room temperature. Since the room temperature strength limits (i.e., yield and ultimate stress) are lower than for cryogenic temperatures and the operating margin is smaller here, the room temperature limits were studied in more detail. The stress analysis was performed in the presence of the slow tuner and other appurtenance loads. The final design exceeds all evaluation criteria for the niobium and the stainless steel (SS) parts, respectively. Two methods have been studied for minimization of the cavity frequency sensitivity to fluctuations of the helium pressure: (1) adding gusseting to reduce the cavity deflections in the high magnetic and electric field regions, and (2) varying the depth of the flat dish located opposite to the RF coupler port. The results of these studies showed that no gusseting is required; a minimal value of $1.4 \mathrm{kHz} / \mathrm{atm}$ was achieved by optimizing the dimensions of the flat dish penetration. Simulations of the slow tuner were performed by applying a force to the SS flanges of the helium jacket. For example, a $10 \mathrm{kN}$ force results in a frequency shift of -120 $\mathrm{kHz}$.

The primary operational parameters for the HWR presented in Tables $2.3-2.5$ are based on experience with the ATLAS energy upgrade cryomodule and its long term operation [72], and recent tests of the first undressed HWR cavities. As shown in Figure 3.10, the tests of ATLAS 72 $\mathrm{MHz}$ Quarter Wave resonators (QWR) show $\sim 2 \mathrm{n} \Omega$ residual surface resistance at $48 \mathrm{mT}$, which readily supports design parameters of the HWRs. Recent measurements of the two first HWR cavities showed $\mathrm{Q}_{0}$ equal to $1.7 \cdot 10^{10}$ at the operating gradient which corresponds to a surface 
resistance of $2.8 \mathrm{n} \Omega$. Although this value is slightly larger it actually is a more optimistic value for the surface resistance if one takes into account the 2.2 times increase in the operating frequency. Thus measured $\mathrm{Q}_{0}$ value provides a margin of more than 3 times relative to a conservative value of $\mathrm{Q}_{0}$ presented in Table 2.6. Note that the ATLAS cavities were measured in a real cryomodule while the HWR cavities in the test-stand. However experience accumulated in recent years assures us that there is no significant $\mathrm{Q}_{0}$ increase when cavity is moved to a cryomodule.
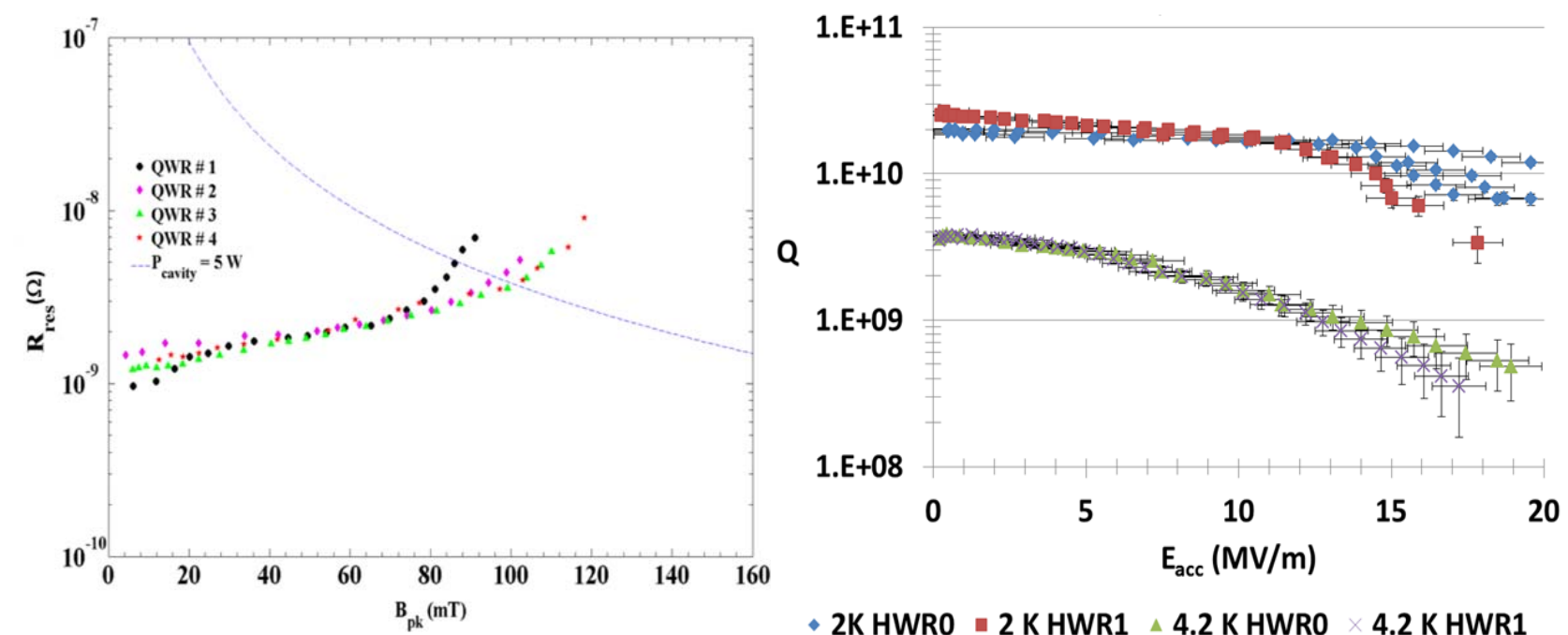

- 2K HWRO $=2 \mathrm{~K}$ HWR1 $4.2 \mathrm{KHWRO} \times 4.2 \mathrm{KHWR1}$

Figure 3.10: Cavity residual resistance measured in the ANL Intensity Upgrade QWR (left) and recent measurements of $\mathrm{Q}_{0}$ dependence on the accelerating gradient for the first two HWR cavities (right); operating accelerating gradient is $9.7 \mathrm{MV} / \mathrm{m}$, and corresponding peak magnetic field - 48 $\mathrm{mT}$.

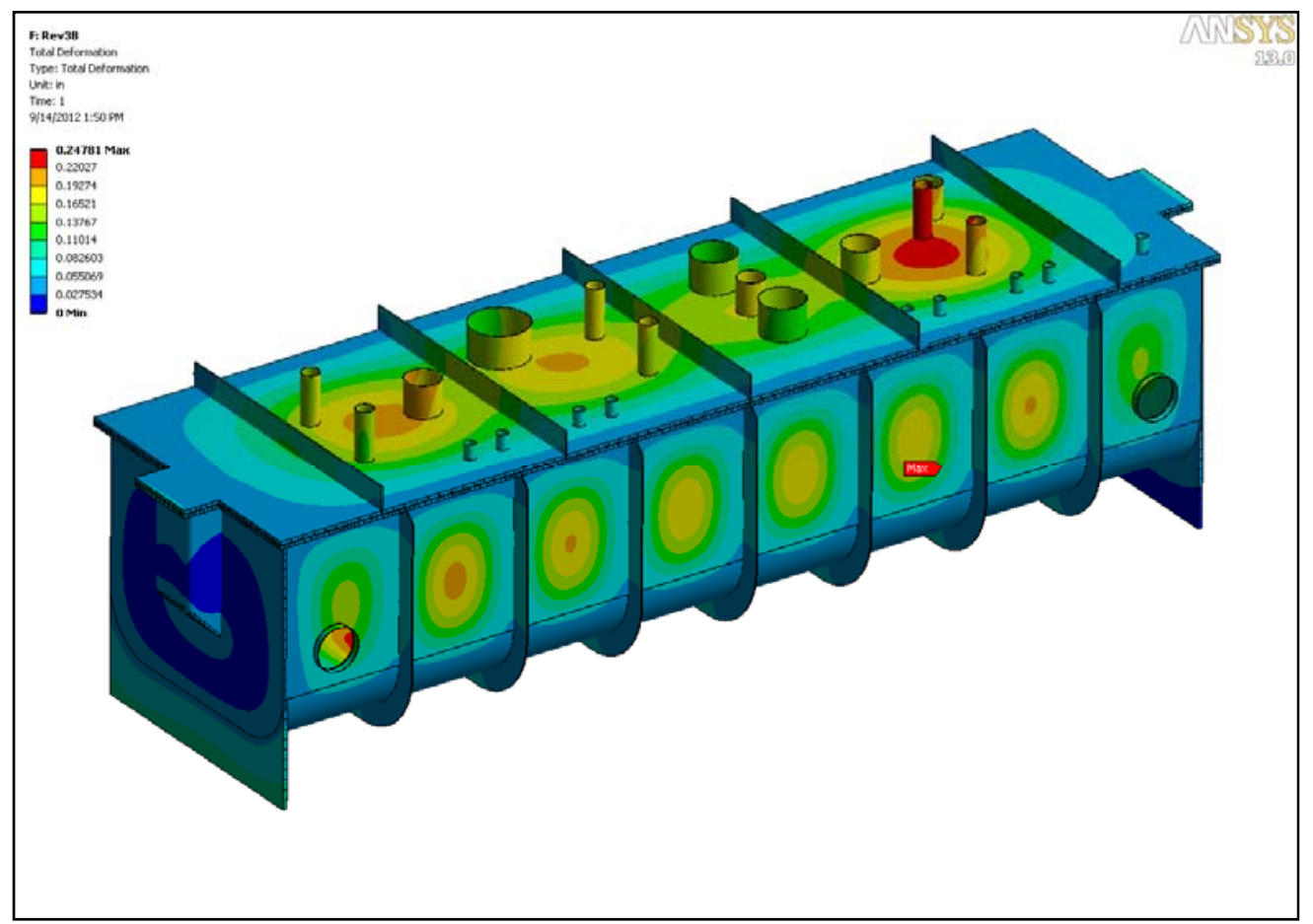

Figure 3.11: ANSYS results of the vacuum vessel deformation due to a 14.7 psi static pressure gradient across the walls. The red color corresponds to displacements greater than 0.6 inches with the maximum being 0.67 inches. 
The cryomodule designs all build upon past ANL experience with box cryomodules. In the HWR case the cryomodule is much wider due to the half-wave cavities being mounted on their sides. To keep a half-cylinder bottom would make the vacuum vessels unacceptably tall. We have arrived at making the vacuum vessel a box which appears to be a good compromise between fabrication cost, structural integrity and minimizing cryostat height. The radii of the rounded corners were chosen to fit the contents of the box minimizing the overall height including the depth of the required gussets. Figure 3.11 shows the results of ANSYS calculations of the structural deformations due to vacuum resulting in walls being pulled to the inside. Notice that the structure pulls in about 0.25 " on average due to evacuation, the maxima are between 0.5 " and 0.67 ". Motion of the vacuum vessel wall moves the internal magnetic shielding and stresses the baton points which may degrade performance. Reducing the maximum displacement to less than 0.25 " will avoid this but it adds the cost of additional gusseting. Future tests are planned to evaluate the magnetic shielding.

The cryomodule houses 8 sets of identical components. Each set forms a focusing period and includes a resonator, a SC solenoid with 4 dipole coils and a Beam Position Monitor (BPM). Beam dynamics requires the solenoids to be aligned to better than $\pm 0.5 \mathrm{~mm}$ peak transversely with $\pm 0.1^{0}$ for all of the rotation angles with similar constraints on the cavities. The beam-line string length is 6 meters and will be supported and aligned on a cryomodule spanning titanium rail system, called the strong-back as shown in Figure 3.12. The strong-back is composed of 2 inch $\times 8$ inch grade 2 titanium plates formed into a box and supported by titanium hangers. Each component is mounted on top of the strong back with its own independent kinematic-alignment hardware.

Table 3.2 summarizes the estimated static and dynamic heat loads at each temperature level in the cryomodule assembly (Figure 3.13) from all sources. The following sources were included in the calculation of $2 \mathrm{~K}$ heat load: cavities, RF couplers, helium manifold, radiation from $70 \mathrm{~K}$ to $2 \mathrm{~K}$, instrumentation, high current leads, strongback hangers, cavity and solenoid cooldown lines, vacuum manifold, slow tuners, and gate valves. Changing the operating voltages by + and $-20 \%$ will result to $28 \mathrm{~W}$ and $21 \mathrm{~W}$ total $2 \mathrm{~K}$ heat load respectively. Currently two HWR prototypes are being fabricated. In addition, a high-power RF coupler, a BPM and SC solenoid were built and cold tested.

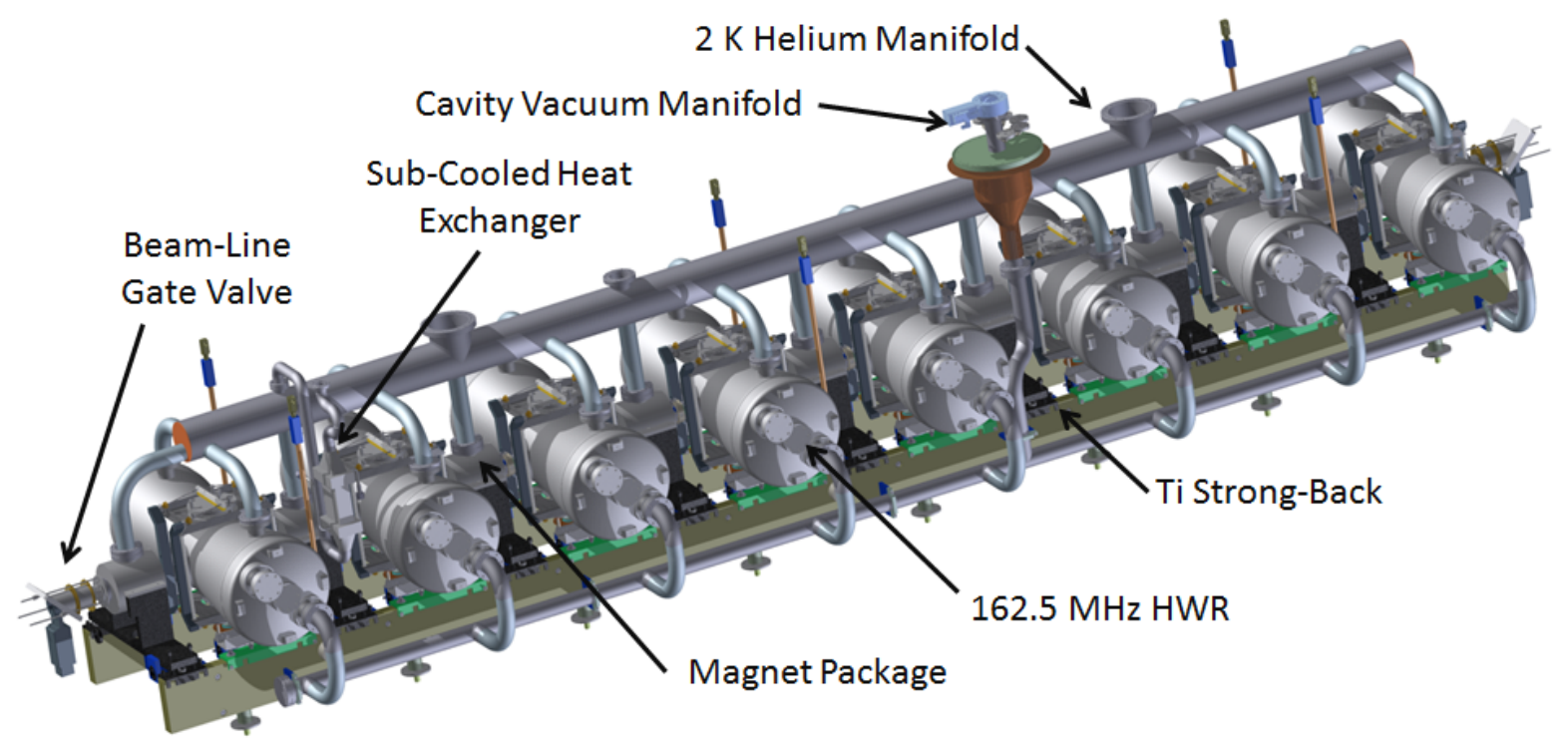

Figure 3.12: HWR Cavity String Assembly. 
Table 3.2: HWR Cryomodule Heat Load Estimate

\begin{tabular}{l|l}
\hline \hline Temperature & Load, $\mathrm{W}$ \\
\hline $2 \mathrm{~K}$, static & 14 \\
\hline $2 \mathrm{~K}$, dynamic & $12^{*}$ \\
\hline $5 \mathrm{~K}$ & 60 \\
\hline $70 \mathrm{~K}$ & 250 \\
\hline \hline
\end{tabular}

*This value takes into account actual voltage distribution on the HWR cavities

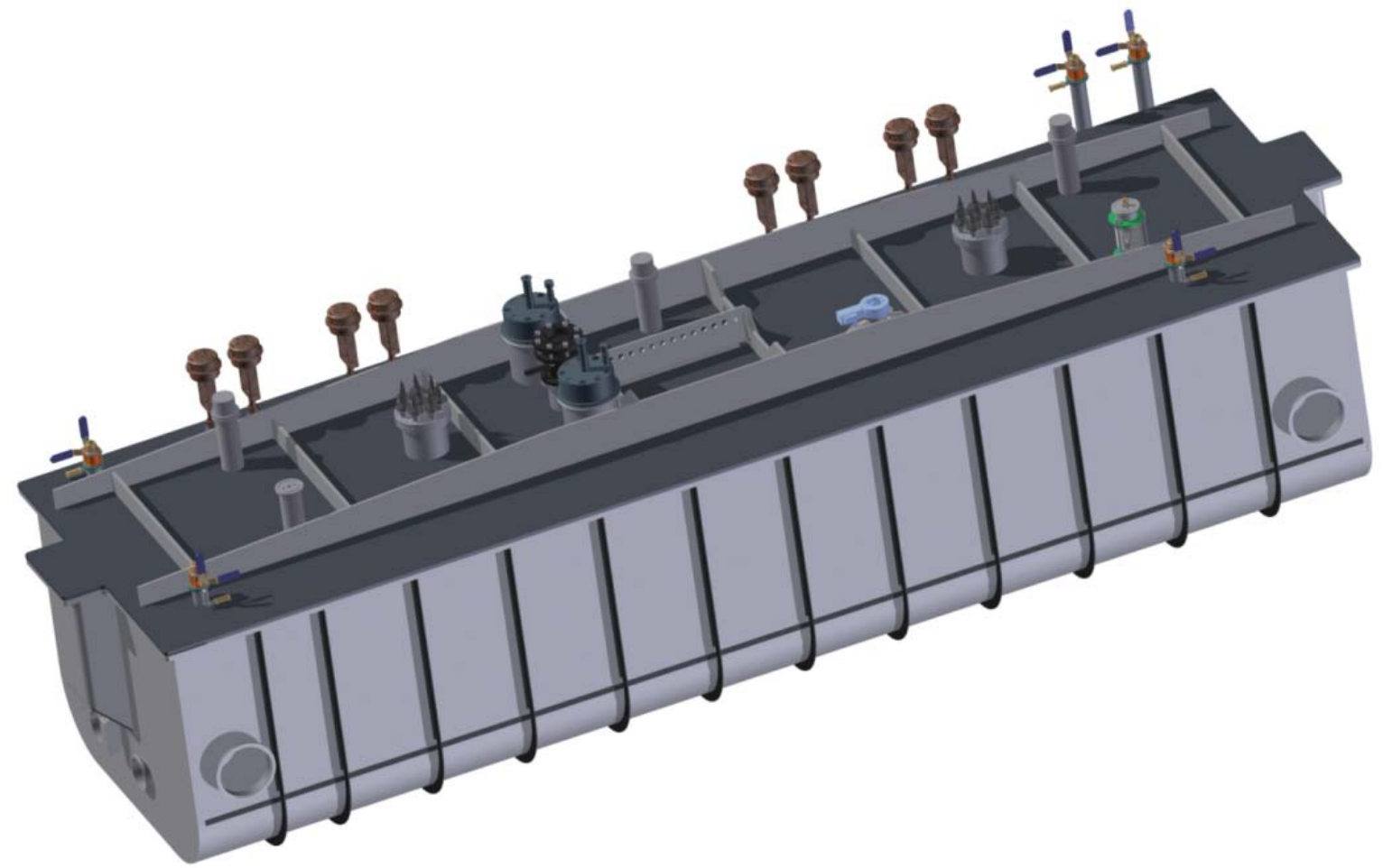

Figure 3.13: HWR cryomodule assembly.

\subsubsection{Single Spoke Resonator I (SSR1) Cavities and Cryomodules}

Two $325 \mathrm{MHz}$ cavity types are required to accelerate beam from 10 to $185 \mathrm{MeV}$ ( $\beta=0.15$ to $0.63)$. They are named SSR 1 and SSR2. The general requirements on their parameters are listed in Tables $2.3-2.5$.

\section{SSR1 Cryomodule}

Acceleration from 10 to $35 \mathrm{MeV}$ utilizes superconducting SSR cavities with $\beta_{\text {opt }}=0.222$ (SSR1). The cavity has geometrical and electro-magnetic parameters shown in Tables $2.3-2.5$. A SSR1 cavity matching these requirements has been designed, fabricated, and tested with RF power as part of the HINS program. The mechanical design, including focusing elements, is displayed in Figure 3.14.

Figure 3.15 shows the first (SSR1-02) cavity fabricated as part of the HINS program. The left photograph shows the bare cavity, the right a "dressed" cavity encased in its He jacket with ancillary slow and fast (piezo) tuners. To date an additional ten bare cavities were fabricated and delivered to Fermilab, (SSR1-05 - SSR1-14). All of them have been tested and showed parameters 
suitable for operation in PIP-II. The measured performance at $2 \mathrm{~K}$ of the bare cavity in a vertical test is displayed in Figure 3.16. Note that the cavities are made from niobium, which is not certified for high-gradient ILC operation due to demonstrated higher losses than material from certified vendors. However, all the cavities show a $\mathrm{Q}_{0}>7 \times 10^{9}$ at the $2 \mathrm{~K}$ at the operating gradient of 10 $\mathrm{MeV} / \mathrm{m}$, which is well above the required value $\mathrm{Q}_{0}>6 \times 10^{9}$. Note that the cavity SSR1-02 made of certified material demonstrated a $\mathrm{Q}_{0}=1.1 \times 10^{10}$ at $2 \mathrm{~K}$ at the operating gradient. The measured surface resistance of this cavity as a function of temperature is shown in Figure 3.16. The cavity operational and test requirements are summarized in Table 3.3.

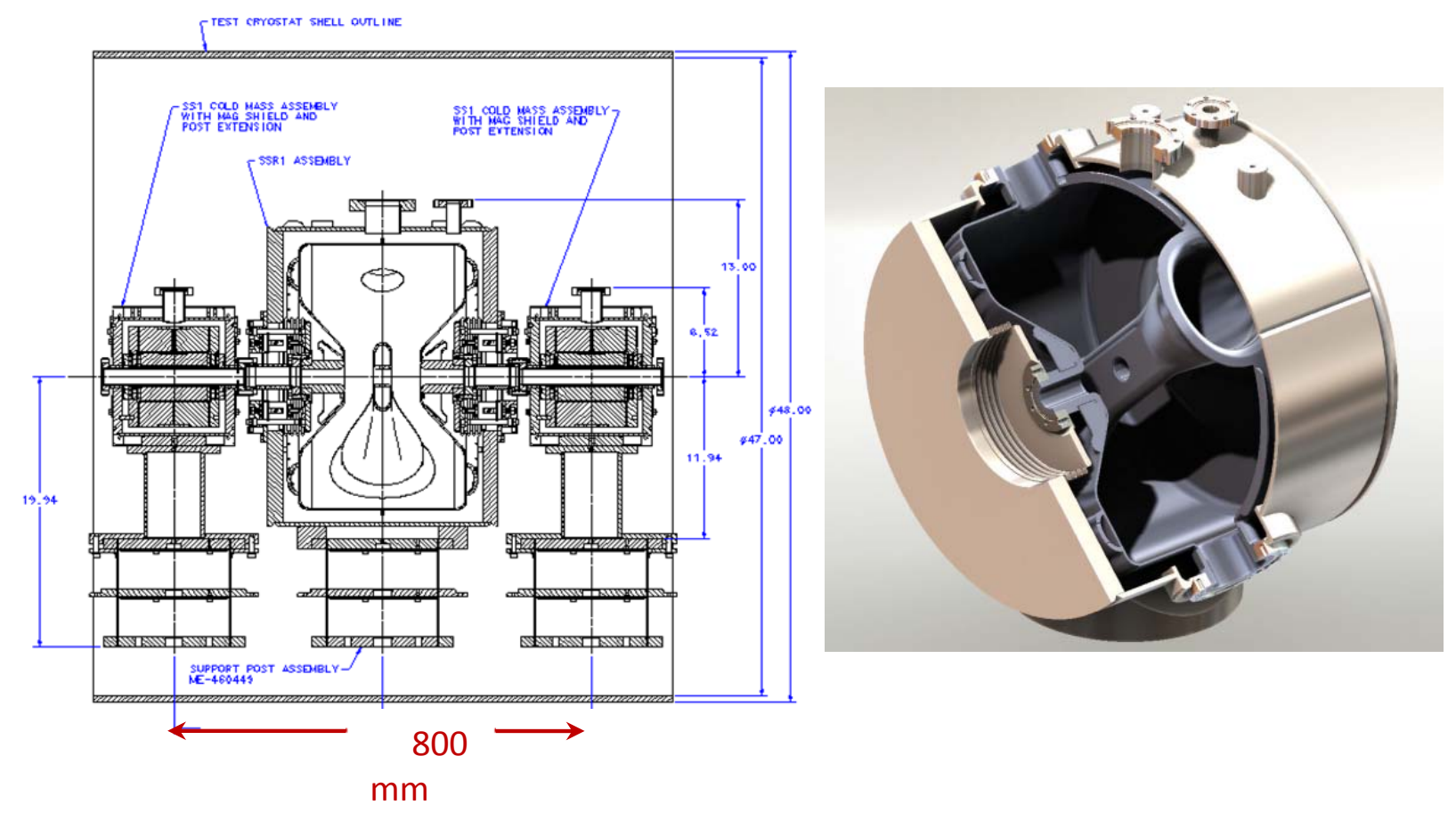

Figure 3.14: SSR1 cavity mechanical design and cutaway view.
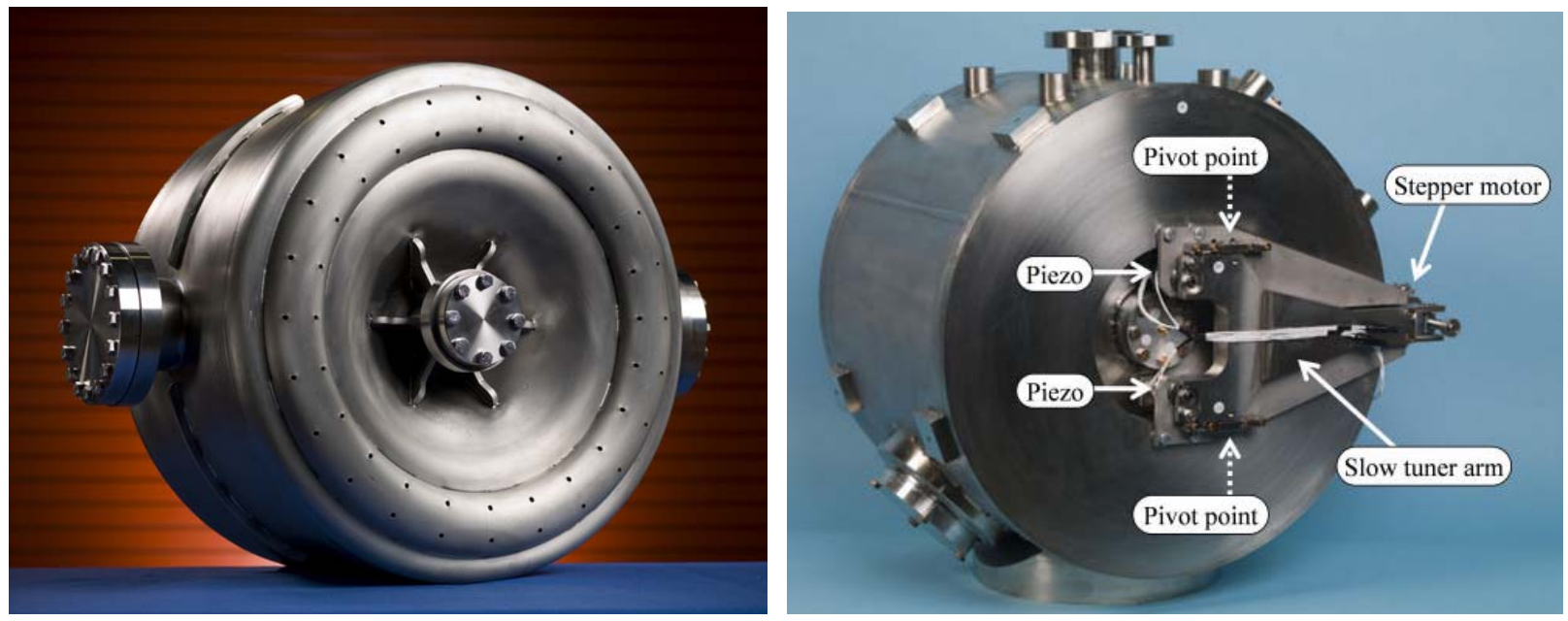

Figure 3.15: Photographs of the bare and dressed prototype SSR1 cavity 


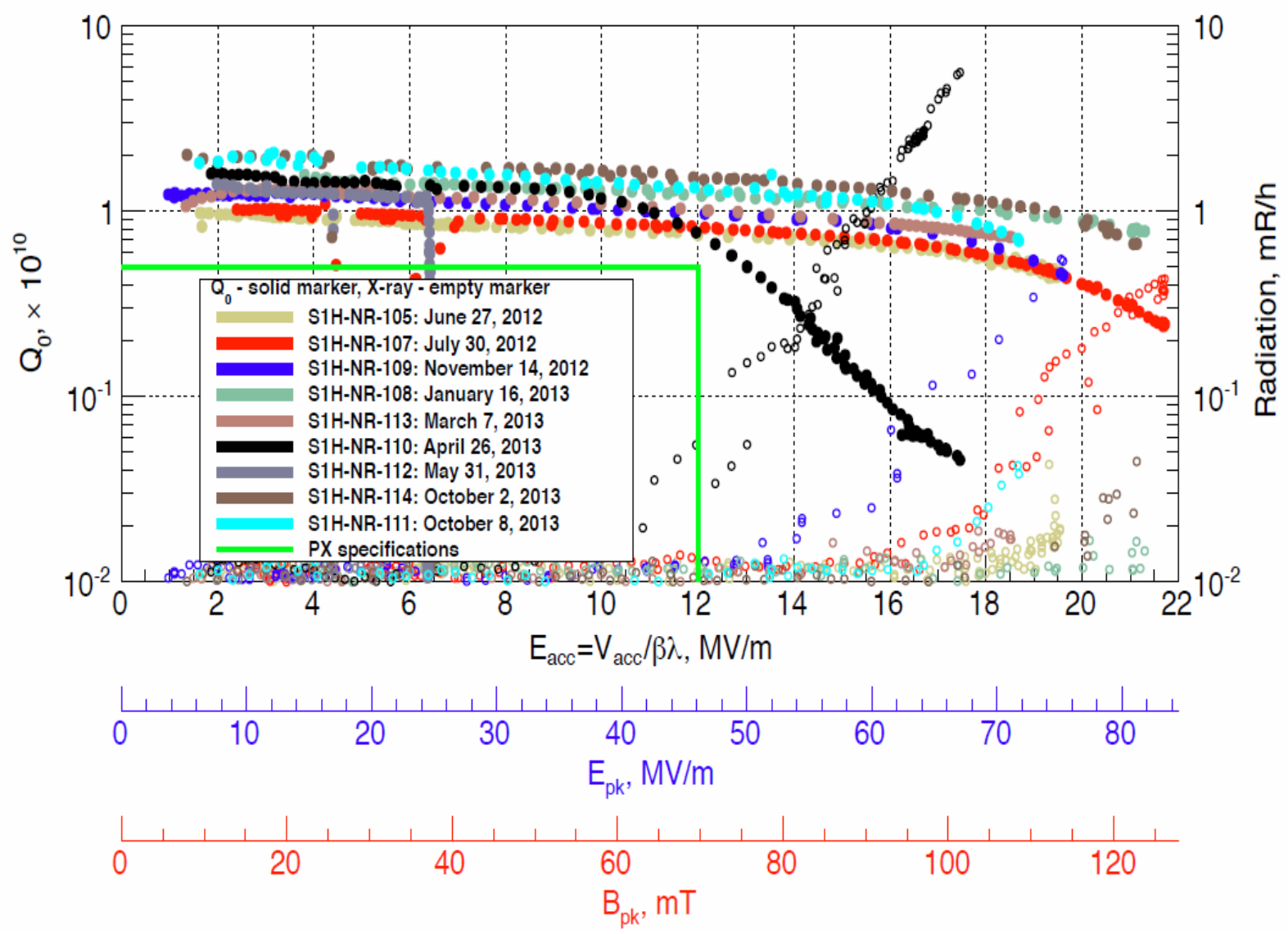

Figure 3.16: $\mathrm{Q}_{0}$ vs. acceleration gradient from the cold test of the nine SSR1 cavities $(\beta=0.222)$. Operating voltage of $2.05 \mathrm{MeV}$ corresponds to $B_{\text {peak }}=58.1 \mathrm{mT}$.

A spoke cavity has no axial symmetry. Therefore its quadrupole component cannot be compensated over the entire range of cavity operation. Figure 2.28 presents the dependence of the quadrupole effect on the beam velocity. Due to engineering limitations, mainly related to the RF couplers, the cavities are rolled by $45^{\circ}$; consequently, their quadrupole field is also rolled and is equivalent to a skew-quadrupole field. The cavity skew-quadrupole fields will be compensated by correction coils located inside nearby focusing solenoids and capable to create dipole and skewquadrupole fields.

In order to attain the requirements for frequency range and resolution (Table 3.4), the tuning systems for cavities of narrow bandwidths such as SSR1 typically integrate coarse and a fine mechanisms engaged in series. The first normally utilizes a stepper motor with large stroke capability and limited resolution, the latter usually contains piezo-electric actuators with limited stroke but virtually infinite resolution.

The coarse tuner is predominantly used to achieve consistently the resonant frequency during the cool-down operations. The range necessary to compensate for cool-down uncertainties is estimated to be $50 \mathrm{kHz}$. In the event that a cavity must be detuned as a result of a malfunction, the coarse tuning system must be able to shift the frequency away from resonance by at least 100 bandwidths, which equals to $\approx 10 \mathrm{kHz}$, so that the beam is not disturbed. The requirement on the range was set arbitrarily considering a safety margin of 2.7 . The requirement on the resolution of the coarse tuning system is set to a value that would allow operation in the event of a failure of the fine-tuning system. Based on other applications, it is believed that such resolution can be achieved 
with a coarse tuning system.

Table 3.3: SSR1 cavity operational and test requirements.

\begin{tabular}{l|l}
\hline \hline Parameter & Requirement \\
\hline Max leak rate (room temp) & $<10^{-10}$ atm-cc/sec \\
\hline Operating gain per cavity & $2.0 \mathrm{MeV}$ \\
\hline Maximum gain per cavity & $2.4 \mathrm{MeV}$ \\
\hline Max. power dissipation per cavity at $2 \mathrm{~K}$ & $5 \mathrm{~W}$ \\
\hline Sensitivity to He pressure fluctuations $d f / d P$ & $<25 \mathrm{~Hz} /$ Torr \\
\hline Field flatness & Within $\pm 10 \%$ \\
\hline Multipacting & None within $\pm 10 \%$ of operating grad. \\
\hline Operating temperature & $1.8-2.1 \mathrm{~K}$ \\
\hline Operating pressure & $16-41 \mathrm{mbar}$ differential \\
\hline MAWP & 2 bar $(\mathrm{RT}), 4$ bar $(2 \mathrm{~K})$ \\
\hline RF power input per cavity & $6 \mathrm{~kW}(\mathrm{CW}$, operating) \\
\hline \hline
\end{tabular}

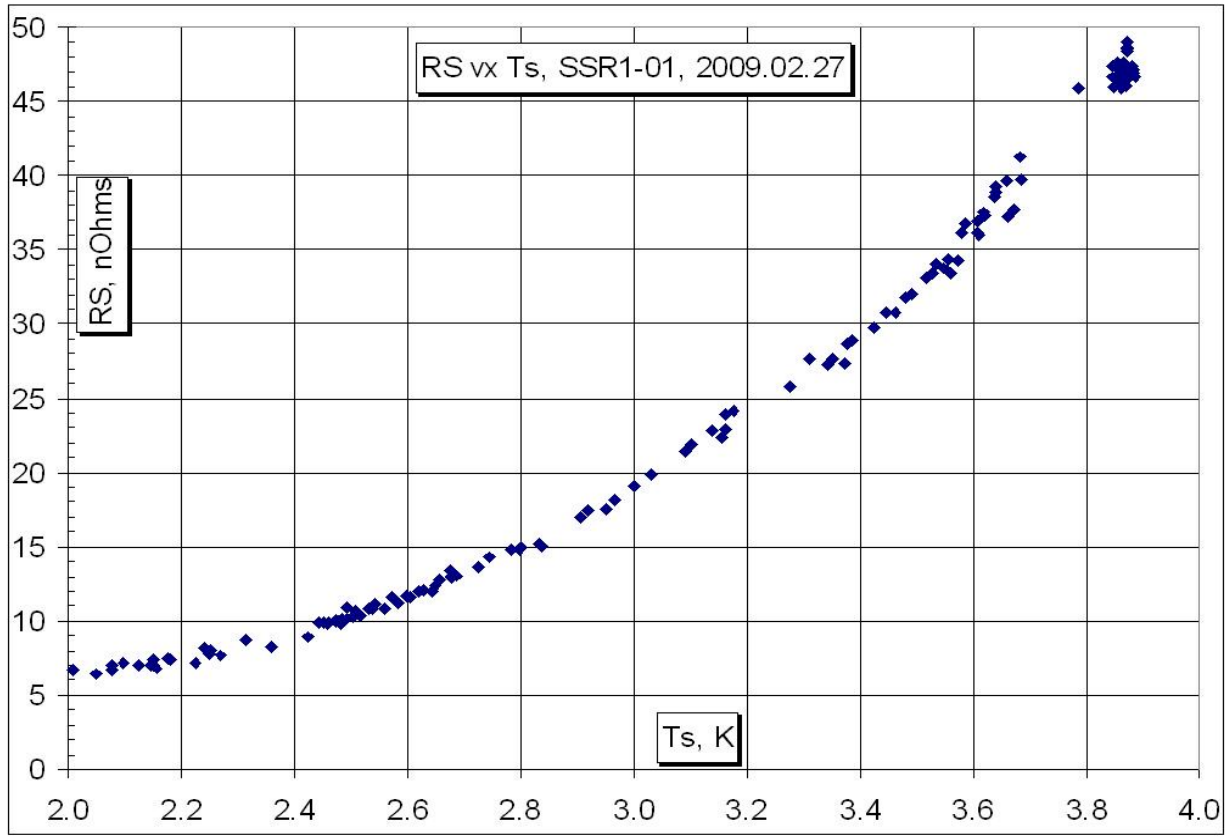

Figure 3.17: Temperature dependence of the surface resistance for SSR1 cavity.

Table 3.4: SSR1 tuning system requirements

\begin{tabular}{l|l}
\hline \hline & Requirement \\
\hline Coarse frequency range & $135 \mathrm{kHz}$ \\
\hline Coarse frequency resolution & $20 \mathrm{~Hz}$ \\
\hline Fine frequency range & $1 \mathrm{kHz}$ \\
\hline Fine frequency resolution & $\leq 2 \mathrm{~Hz}$ \\
\hline \hline
\end{tabular}


It is conservatively assumed that the coarse system cannot be operated during beam acceleration; it is thought that the vibration of a stepper motor may induce vibrations in the cavity severe enough to disrupt the operation. Thus, fine tuners shall be designed to compensate, at a minimum, the frequency shifts of the cavity induced by fluctuations of the helium bath pressure. The use of fine tuners will reduce considerably the hysteresis of the system by limiting the elements in motion during the tracking of the frequency. A particular design effort shall be dedicated to facilitate access to all actuating devices of the tuning system from access ports on the vacuum vessel. All actuating devices must be replaceable from the ports, either individually or as a whole cartridge.

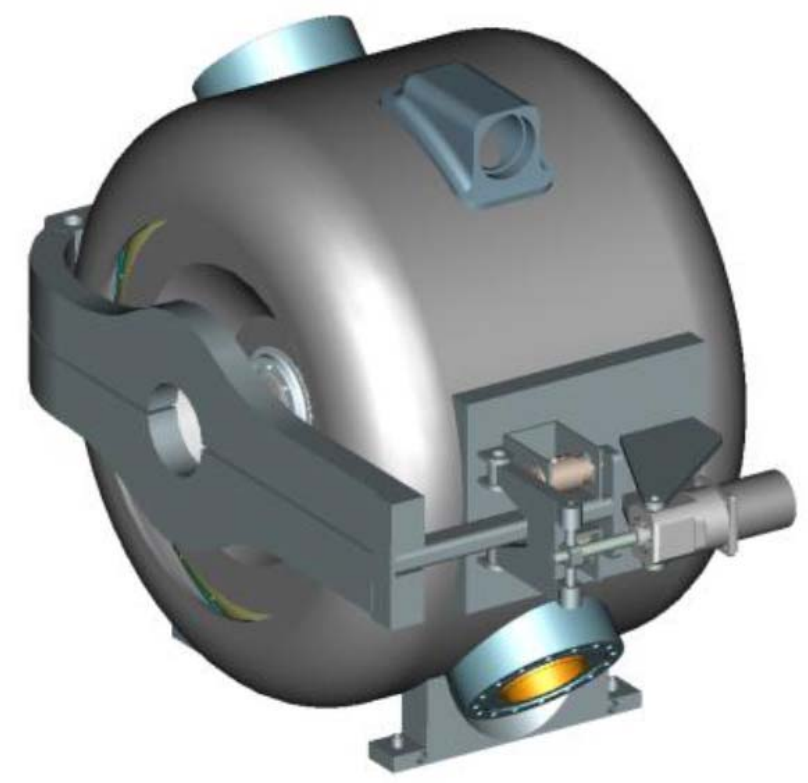

Figure 3.18: SSR1 cavity, helium vessel, and tuner.

The Helium vessel is fabricated from a non-magnetic stainless steel (e.g. 316L) designed to house a $2 \mathrm{~K}$ helium bath sufficient to remove up to 5 watts average dissipated power, with appropriately sized supply and return piping. It meets the requirements of the Fermilab ES\&H Manual for cryogenic pressure vessels and is rated at an MAWP (Maximum Allowable Working Pressure) of no less than 2 bar at room temperature and 4 bar at $2 \mathrm{~K}$. The cavity vessel with tuner system is shown in Figure 3.18.

\section{SSR1 Current Leads}

Each focusing element package contains five coils: the main solenoid, operating up to $100 \mathrm{~A}$, and four coils which can be combined to serve as both $x$ and $y$ steering and skew-quadrupole correctors. Each coil can operate up to $50 \mathrm{~A}$. A conduction cooled current lead design modeled after similar leads installed in the LHC at CERN is being developed for use in the SSR1 cryomodule. Thermal intercepts at $70 \mathrm{~K}$ and at $5 \mathrm{~K}$ help reduce the heat load to $2 \mathrm{~K}$, nonetheless, these current leads represent a significant source of heat at the low temperature end. There will be one lead assembly for each magnetic element.

\section{SSR1 Solenoid and Beam Position Monitor}

The four magnet packages in the cryomodule each contain a focusing solenoid (lens) and four corrector coils all operating in a helium bath at $2 \mathrm{~K}$. The general design requirements for the lenses 
in the SSR1 cryomodule are summarized below.

Requirements essential for the beam dynamics in the linac:

- The integrated focusing strength of the lens must be not less than $4 \mathrm{~T}^{2} \mathrm{~m}$;

- Each lens must contain 4 coils which can be combined into two dipole correctors; bending strength of each corrector must be not less than $0.0025 \mathrm{~T}-\mathrm{m}$;

- The clear aperture in the lens must be not less than $30 \mathrm{~mm}$;

- The uncertainty in the location of the effective magnetic axis in the focusing solenoid of the lens relative to reference points on the outer surface of the device must be better than $0.1 \mathrm{~mm}$ rms.

Requirements essential for proper functioning of the cryomodule:

- Maximum current in the solenoid must be less than $100 \mathrm{~A}$;

- Maximum current in the dipole correctors must be less than $50 \mathrm{~A}$;

- A LHe vessel must be used for cooling the windings down to $2 \mathrm{~K}$;

- The lenses must be quench-protected; the energy deposited in the lenses after quenching must be as low as reasonably achievable;

- The LHe vessel must meet the requirements of the Fermilab's ES\&H manual chapters for pressure vessel;

- The design of the LHe vessel must ensure reliable and reproducible mechanical connection to the alignment fixture of the cryomodule;

- The maximum magnetic field generated by lenses in the cryomodule in the area near the surface of the SSR1 superconducting cavities must not exceed the level that would result in more than two-fold reduction of the intrinsic quality factor after quench event at any point on the surface of the cavity.

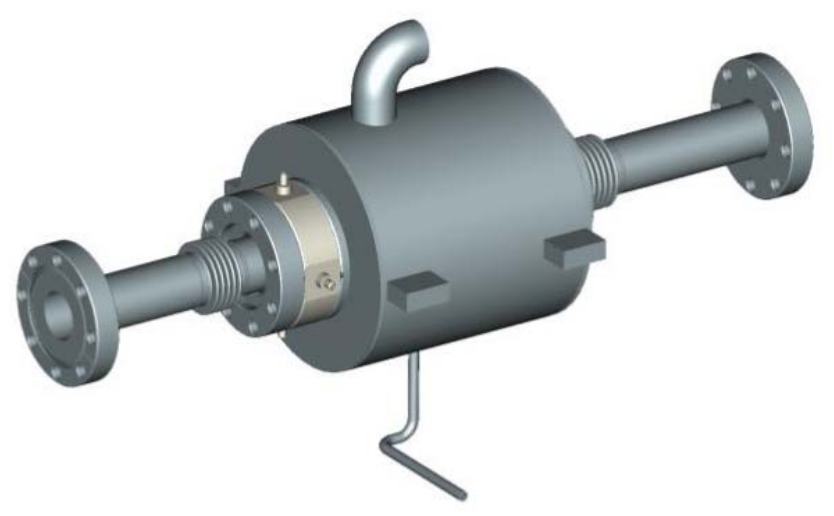

Figure 3.19: Solenoid and BPM assembly.

The linac lattice, especially the low-beta section, provides limited space along the beamline for beam diagnostics either inside individual cryomodules or between adjacent modules. In order to conserve axial space along the beamline a button-type beam position monitor (BPM) has been chosen for installation in the SSR cryomodules. For a non-relativistic beam they also generate larger signal than strip-line BPMs. A total of four BPMs will be installed in the cryomodule, one near each magnetic element. These devices are compact and lend themselves well to incorporation into the solenoid magnet package as shown in Figure 3.19. The bellows at either end of the beam 
tube allow independent alignment of each magnet.

\section{Final Assembly}

The final assembly of the SSR1 cryomodule for SSR1 is shown in Figures 3.20 and 3.21. Figure 3.20 shows the cavity string consisting of the cavities, solenoids, beam position monitors, and internal piping mounted on support posts that are in turn mounted to the strongback. Figure 3.21 shows the entire cryomodule assembly.

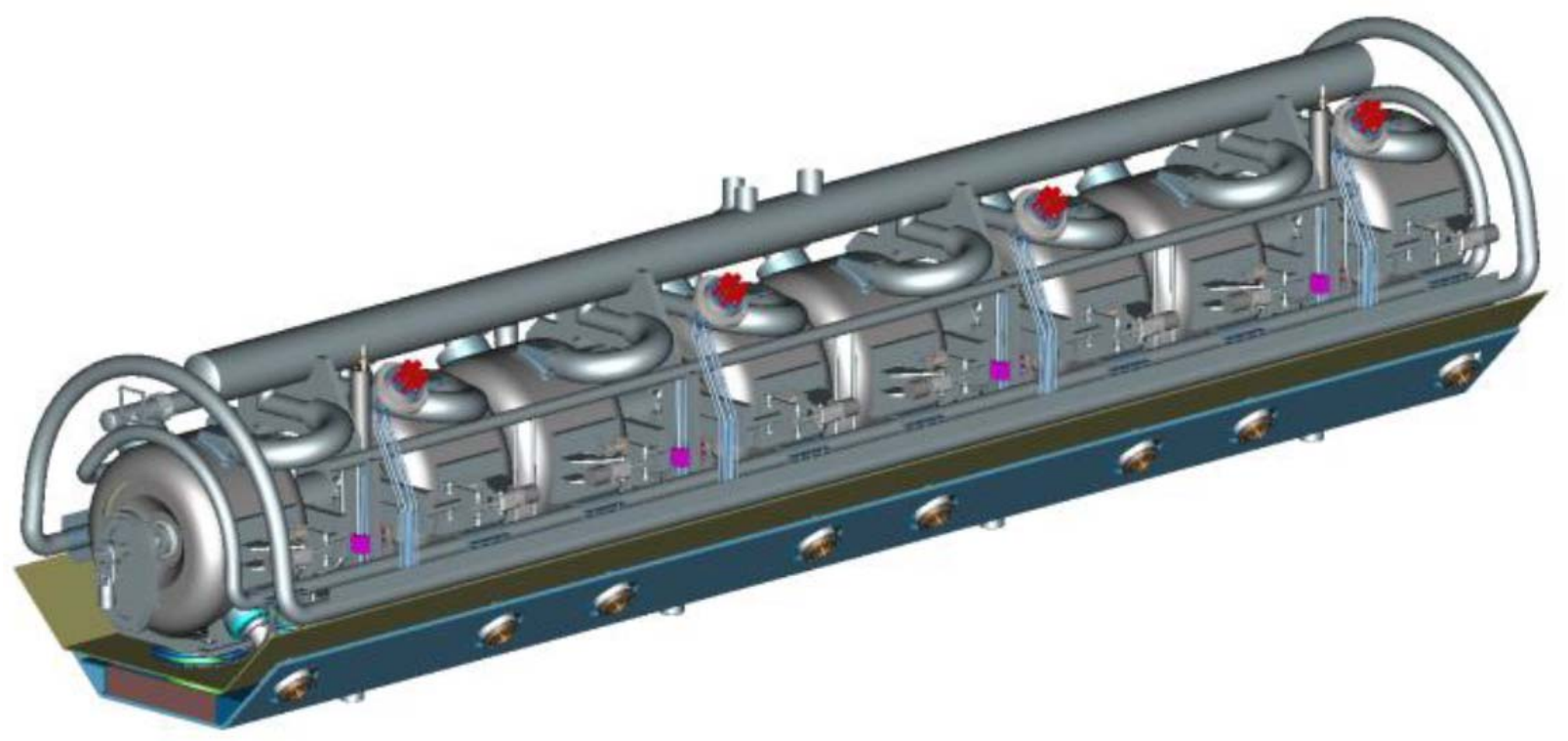

Figure 3.20: SSR1 cavity string assembly.

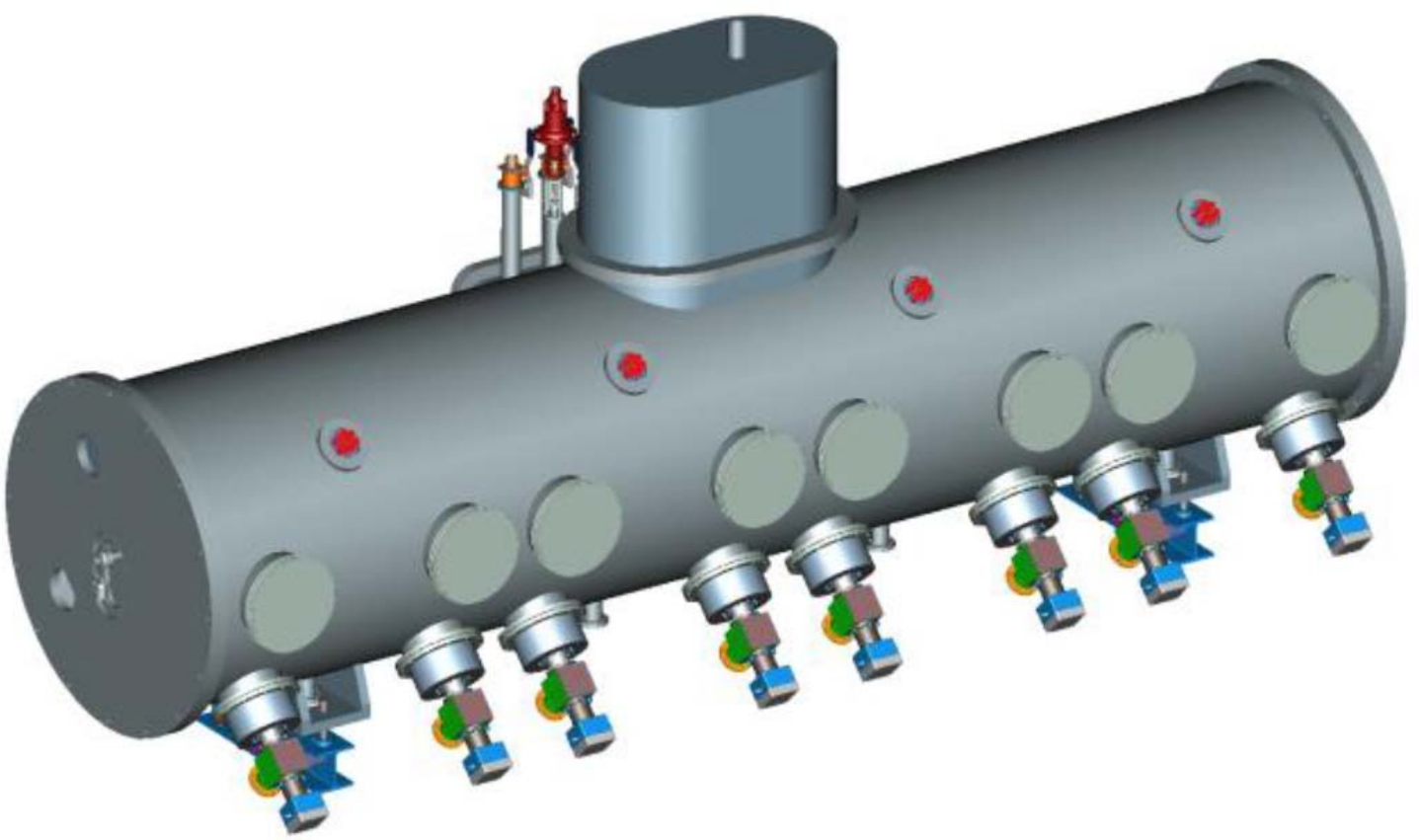

Figure 3.21: SSR1 cryomodule assembly 


\section{SSR1 Heat Load Estimate}

Table 3.5 summarizes the estimated static and dynamic heat loads at each temperature level in the cryomodule assembly from the primary sources. As mentioned earlier, the nominal $70 \mathrm{~K}$ thermal shield and intercepts may operate anywhere between 45 and $80 \mathrm{~K}$.

Table 3.5: SSR1 Cryomodule Heat Load Estimates

\begin{tabular}{l|l|l|l|l|l|l|l}
\hline \multirow{2}{*}{} & \multicolumn{2}{|c|}{ Per Unit (W) } & \multicolumn{2}{l|}{ Units } & \multicolumn{2}{l}{ Total (W) } \\
\cline { 2 - 8 } & $70 \mathrm{~K}$ & $5 \mathrm{~K}$ & $2 \mathrm{~K}$ & & $70 \mathrm{~K}$ & $5 \mathrm{~K}$ & $2 \mathrm{~K}$ \\
\hline Input coupler, static & 5.4 & 2.8 & 0.5 & 8 & 43 & 23 & 4 \\
\hline Input coupler, dynamic & 0 & 0 & 0.25 & 8 & 0 & 0 & 2 \\
\hline Cavity, dynamic & 0 & 0 & 1.8 & 8 & 0 & 0 & 14 \\
\hline Support post & 2.8 & 0.4 & 0.05 & 12 & 33 & 4 & 0.6 \\
\hline Conductor Lead Assembly & 36.8 & 13.2 & 1.2 & 4 & 147 & 53 & 5 \\
\hline MLI & 30.5 & 0 & 1.4 & 1 & 31 & 0 & 1 \\
\hline Cold-warm transition & 0.7 & 0.1 & 0.01 & 2 & 1 & 0.2 & 0.02 \\
\hline TOTAL & & & & & 255 & 80 & 27 \\
\hline \hline
\end{tabular}

* MLI stands for multi-layer thermal insulation.

\subsubsection{Single Spoke Resonator II (SSR2) Cavities and Cryomodules}

Acceleration from 35 to $185 \mathrm{MeV}$ utilizes superconducting SSR cavities with $\beta_{\text {opt }}=0.51$ (SSR2). The cavity geometrical and electro-dynamic and mechanical design parameters are listed in Tables 2.3-2.5. The cavity layout is shown in Figure 3.22.

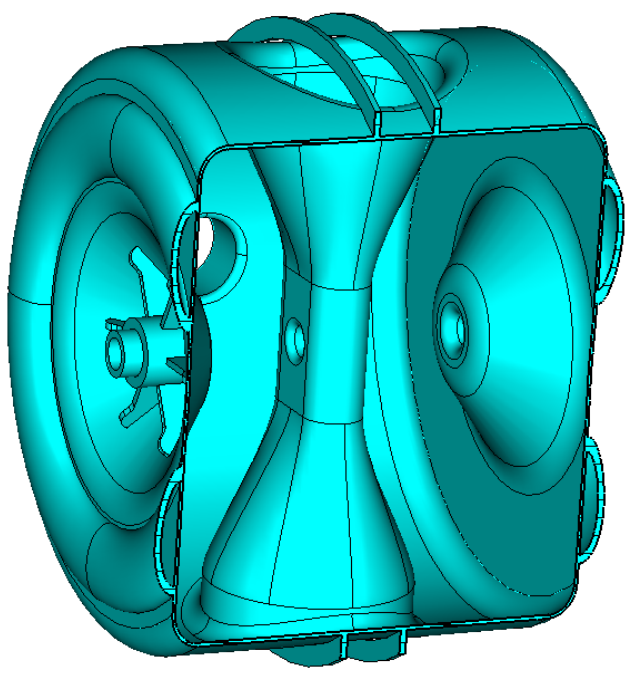

Figure 3.22: SSR2 cavity layout.

Similar to the SSR1 cryomodule, the quadrupole field is compensated by corrector coils which have independent leads. The helium vessel of SSR2 cavity has a design similar to the SSR1 cavity helium vessel. The input coupler and the fine and coarse tuners are the same as for SSR1 cavity. 
Mechanical parameters of the SSR2 cavities are presented in Table 3.6. The SSR2 cryomodule design contains eight identical slots. Each of them can accommodate either an SSR2 dressed cavity or a focusing solenoid with a corrector package and BPM. The SSR2 cryomodule comprises 5 dressed cavities and 3 solenoids. The overall cryomodule length will be approximately $6.5 \mathrm{~m}$. The vacuum vessel diameter will be $1.22 \mathrm{~m}$ (48 inches). Each cryomodule will be configured as a stand-alone unit, i.e. the vacuum vessel ends will be closed and cryogenic connections will be made at each module. Connections for cryogens and cryogenic control valves will be located in a mid-span vacuum vessel extension. The only module-to-module connection will be the beam line. The only beam instrumentation internal to the cryomodule assembly will be BPMs.

Table 3.6: Mechanical parameters of the SSR2 cavities

\begin{tabular}{|l|l|l|}
\hline & Requirements & $280 \mathrm{~mm}, 540 \mathrm{~mm}$ \\
\hline \hline Mechanical & Radius, Length & Stainless Steel \\
\hline & He Vessel Material & 2 bar RT, 4 bar CT \\
\hline & Maximum Allowable Pressure, MAWP & $\leq 25 \mathrm{~Hz} / \mathrm{mbar}$ \\
\hline Coupler & $d f / d p$ & $30 \mathrm{~kW}$ \\
\hline Tuning & Max. design forward power & $135 \mathrm{kHz}$ \\
\hline & Coarse tuning range & $1000 \mathrm{~Hz}$ \\
\hline
\end{tabular}

\subsubsection{Medium-beta Section (LB650 and HB650)}

Acceleration from $185 \mathrm{MeV}$ to $800 \mathrm{MeV}$ will be provided by two families of 5-cell elliptical cavities operating at $650 \mathrm{MHz}$ and designed to $\beta_{G}=0.61$ and $\beta_{G}=0.92$. The cavity shape is optimized to decrease the field enhancement factors (magnetic and electric) in order to improve the interaction between the beam and the cavities. In order to do this, the cavity aperture should be as small as possible subject to the following considerations:

$$
\begin{aligned}
& \text { - } \quad \text { field flatness, } \\
& \text { - } \quad \text { beam losses, } \\
& \text { - } \quad \text { rechanical stability, } \\
& \text { - }
\end{aligned}
$$

The working gradient is chosen to provide a peak surface magnetic field that allows operation below high-field Q-slope, see Figure 2.16. For a frequency of $650 \mathrm{MHz}$ the peak magnetic field should be not greater than $\sim 70 \mathrm{mT}$. In addition we require that the peak surface electric field be lower than $40 \mathrm{MV} / \mathrm{m}$ in order to avoid the risk of strong field emission.

Linear perturbation theory indicates that for given relative errors in the frequencies of cavity cells the field flatness, $\delta E / E$, is determined mainly by the distance, $\delta f$, between the operating frequency and the frequency of the neighboring mode, $\pi(n-1) / n$. Expressing the result in terms of the coupling parameter, $k$, between cells and the number of cells one obtains:

$$
\frac{\delta E}{E} \approx \frac{f_{\pi}}{\left|f_{\pi}-f_{\pi(n-1) / n}\right|} \equiv \frac{f_{\pi}}{\delta f} \approx \frac{(n-1)^{2}}{k} .
$$

Thus, a cavity with fewer cells allows a smaller coupling coefficient, $k$, for a given field flatness. 
For example, the 9-cell ILC cavity has $\delta f / f_{\pi}$ of $6 \times 10^{-4} \quad(k=1.87 \%)$ and for the 5-cell $650 \mathrm{MHz}$ cavity one can take the same $\delta f l f_{\pi}$ at least, yielding $k>0.5 \%$.

The apertures selected for the cavities represents a trade-off between requirements related to the cell-to-cell coupling and beam loss. The $805 \mathrm{MHz}$ superconducting section of the SNS proton linac, which is close to the PIP-II linac in average current, operates with cavities that have an aperture of $83 \mathrm{~mm}$ for the low-beta part and $100 \mathrm{~mm}$ for the high-beta part. Their experience is that these cavities operate with tolerable beam loss at these apertures. Thus, we have adopted similar dimensions for the $650 \mathrm{MHz}$ cavities of PIP-II. In addition, it appears that these apertures will also be adequate to facilitate the required surface processing.

The $650 \mathrm{MHz}$ cavities require sufficient wall thickness to minimize sagging caused by the overall weight. Figure 3.23 shows results of a simulation of the cavity sag caused by its weight as a function of wall thickness for the $650 \mathrm{MHz}$ cavities and the ILC (1300 MHz) cavity. Note that stiffening rings are used for both the ILC and $650 \mathrm{MHz}$ cavities to increase the rigidity of cavities. A requirement of limiting the maximum cavity sag to $120 \mu \mathrm{m}$ (the same as ILC) results in a $4 \mathrm{~mm}$ wall thickness. A small cavity wall slope (designated by $\alpha$ in Figure 3.24) gives more freedom to decrease the field enhancement factors. However, the slope is limited by surface processing and mechanical stability requirements. The chosen slope of about $2^{\circ}$ results in an acceptable value for the field enhancement.

Optimization of the two $650 \mathrm{MHz}$ cavity shapes was based on the constraints discussed above. The cavity performance parameters are summarized in Table 2.5. The physical description of the cavity shapes is displayed in Figure 3.24 and Table 3.7. Requirements for maximum cavity detuning amplitude and cavity sensitivity versus helium pressure fluctuations were discussed in Section 2 (see also Ref. [31]). Note that the $650 \mathrm{MHz}$ cavities have small beam loading, and thus microphonics mitigation is essential. Therefore the cavities are over-coupled; both active and passive means for microphonics compensation are planned to be used [74]. The preliminary mechanical design of the HB650 cavity is shown in Figure 3.25.

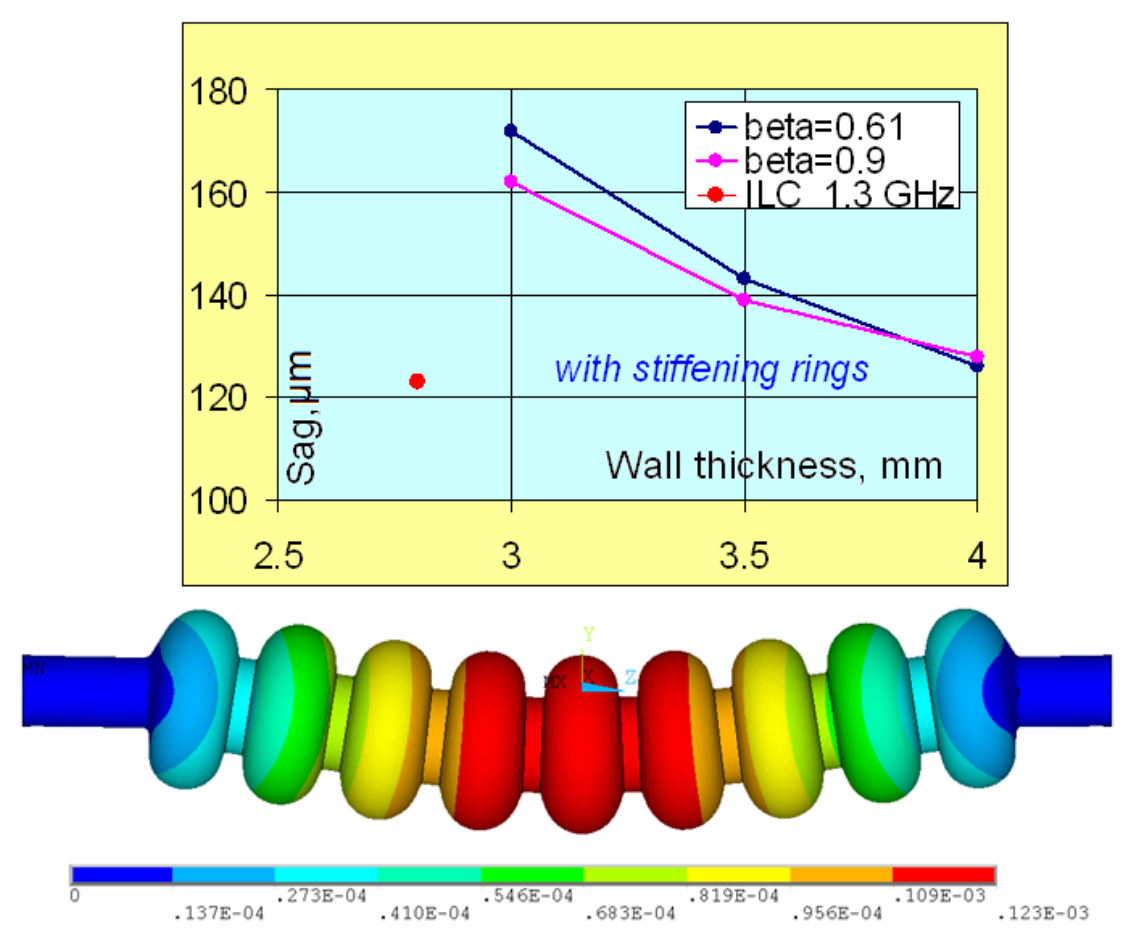

Figure 3.23: The cavity sag versus the wall thickness. 


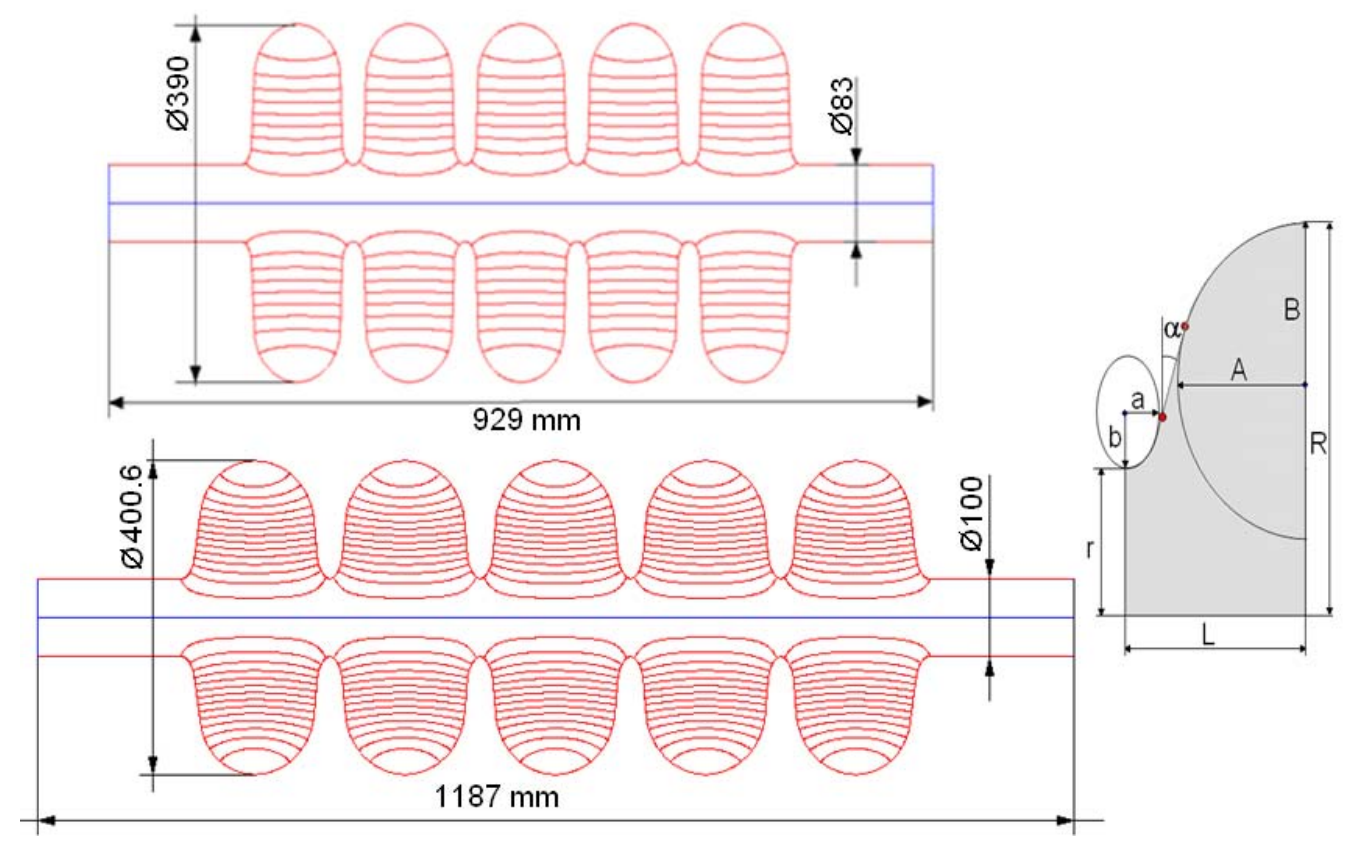

Figure 3.24: Layout of $650 \mathrm{MHz}$ cavities: LB650 - top and HB650 - bottom.

Table 3.7: Dimensions of $650 \mathrm{MHz}$ cavities

\begin{tabular}{l|c|c|c|c}
\hline \hline \multirow{2}{*}{ Dimension* } & \multicolumn{2}{|c|}{ LB650 } & \multicolumn{2}{c}{ HB650 } \\
\cline { 2 - 5 } & Regular cell & End cell & Regular cell & End cell \\
\hline $\mathrm{r}, \mathrm{mm}$ & 41.5 & 41.5 & 59 & 59 \\
\hline $\mathrm{R}, \mathrm{mm}$ & 195 & 195 & 200.05 & 200.05 \\
\hline $\mathrm{L}, \mathrm{mm}$ & 70.3 & 71.4 & 106.1 & 97.6 \\
\hline $\mathrm{A}, \mathrm{mm}$ & 54 & 54 & 85 & 84 \\
\hline $\mathrm{B}, \mathrm{mm}$ & 58 & 58 & 78 & 90 \\
\hline $\mathrm{a}, \mathrm{mm}$ & 14 & 14 & 20 & 13 \\
\hline $\mathrm{b}, \mathrm{mm}$ & 25 & 25 & 33 & 28 \\
\hline$\alpha,^{\circ}$ & 2 & 2.7 & 1.9 & 1.3 \\
\hline \hline
\end{tabular}

* See Figure 3.24 for definition of dimensions.

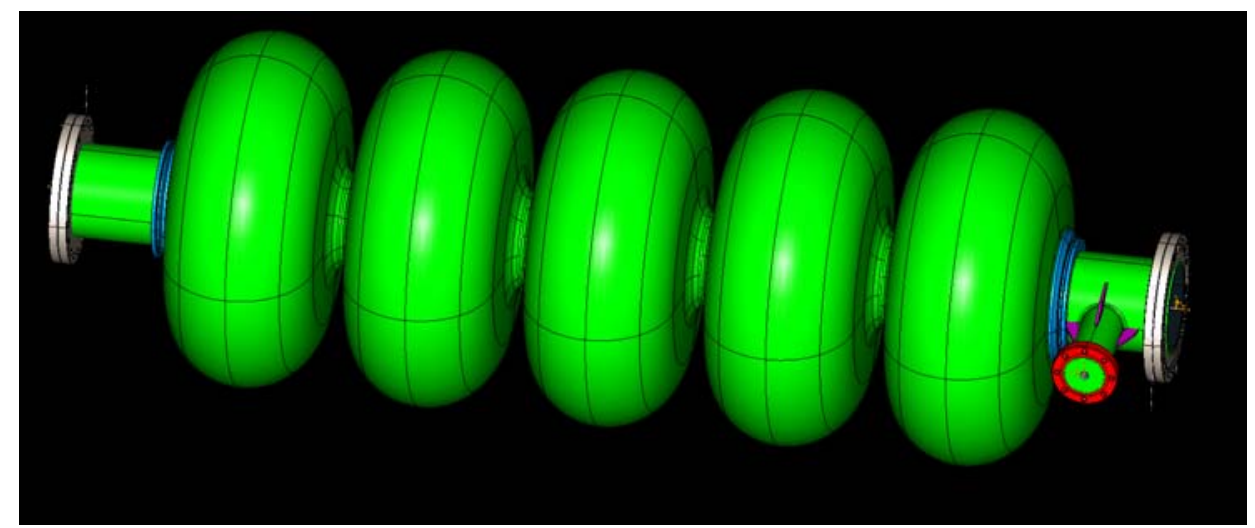

Figure 3.25: Preliminary mechanical design of the HB650 cavity. 


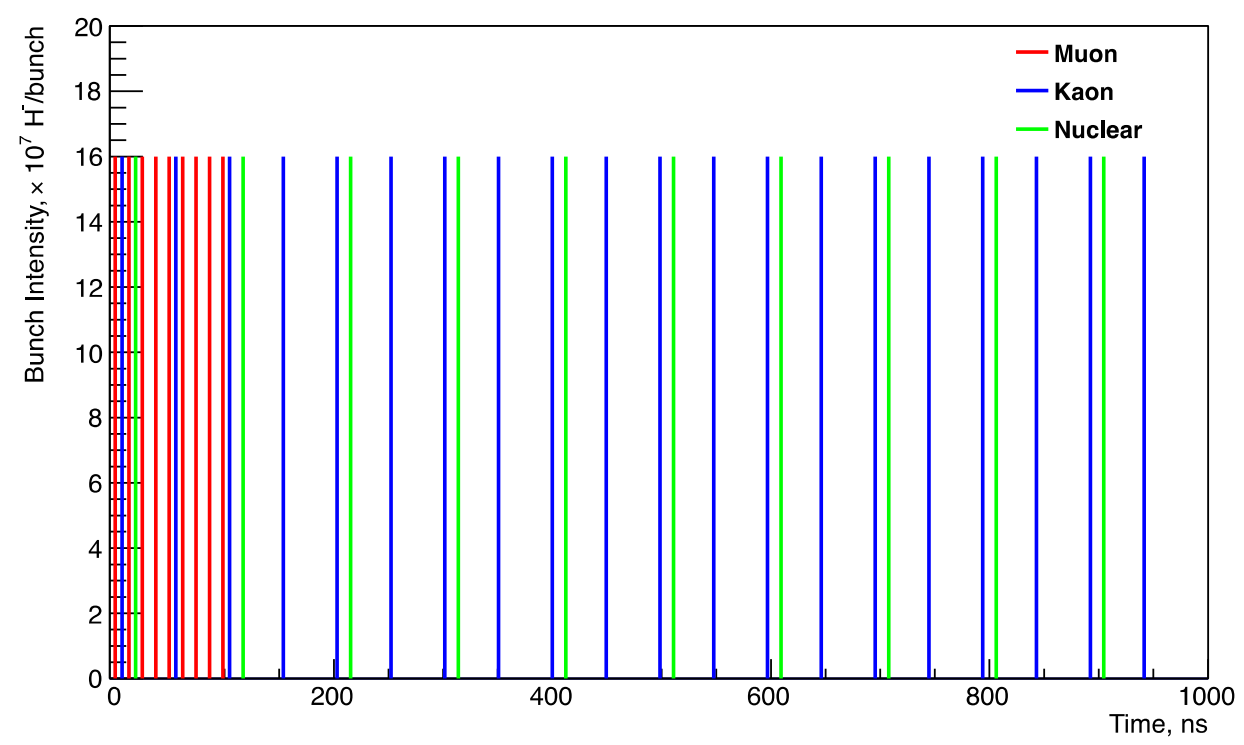

Figure 3.26: Beam structure for $3 \mathrm{GeV}$ program.

Neither HB650 nor LB650 cavities contain HOM dampers - they are not necessary for the required beam current [75]. This choice is also supported by experience accumulated in the SNS [76]. An absence of HOM dampers is more problematic for future PIP-II upgrades. Here we consider the Project $\mathrm{X}$ parameters as an example. Figure 3.26 presents a possible bunch structure considered in Ref. [4] for CW linac operating with $1 \mathrm{~mA}$ average beam current and beam delivery to three experiments running in parallel (muon, kaon, and nuclear experiments) with different beam structure for each experiment. Figure 3.27 shows the corresponding spectrum, assuming very short bunches of equal charge and an absence of timing jitter. The spectrum and $(R / Q)$ values of HB650 cavity are shown in Figure 3.28.

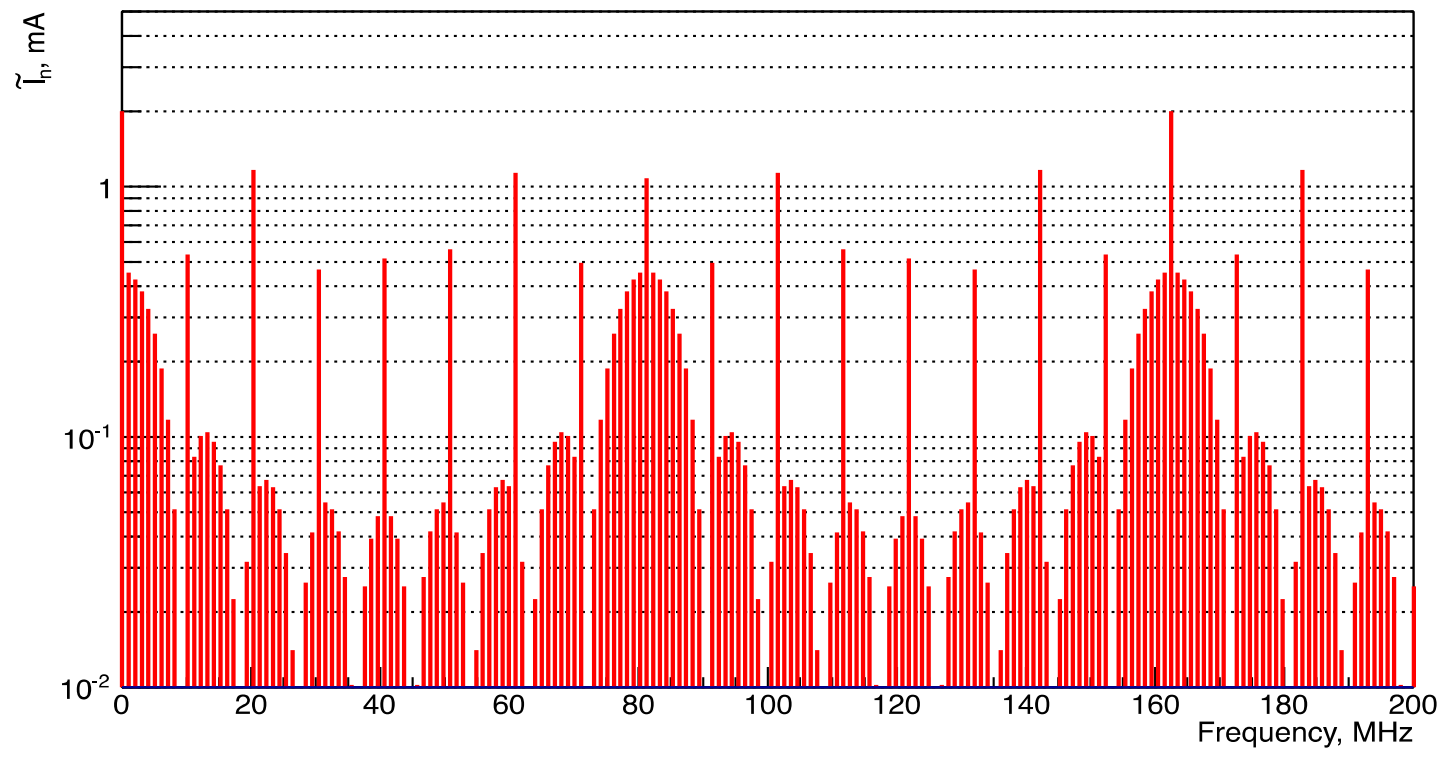

Figure 3.27: Beam spectrum of $3 \mathrm{GeV}$ program. 


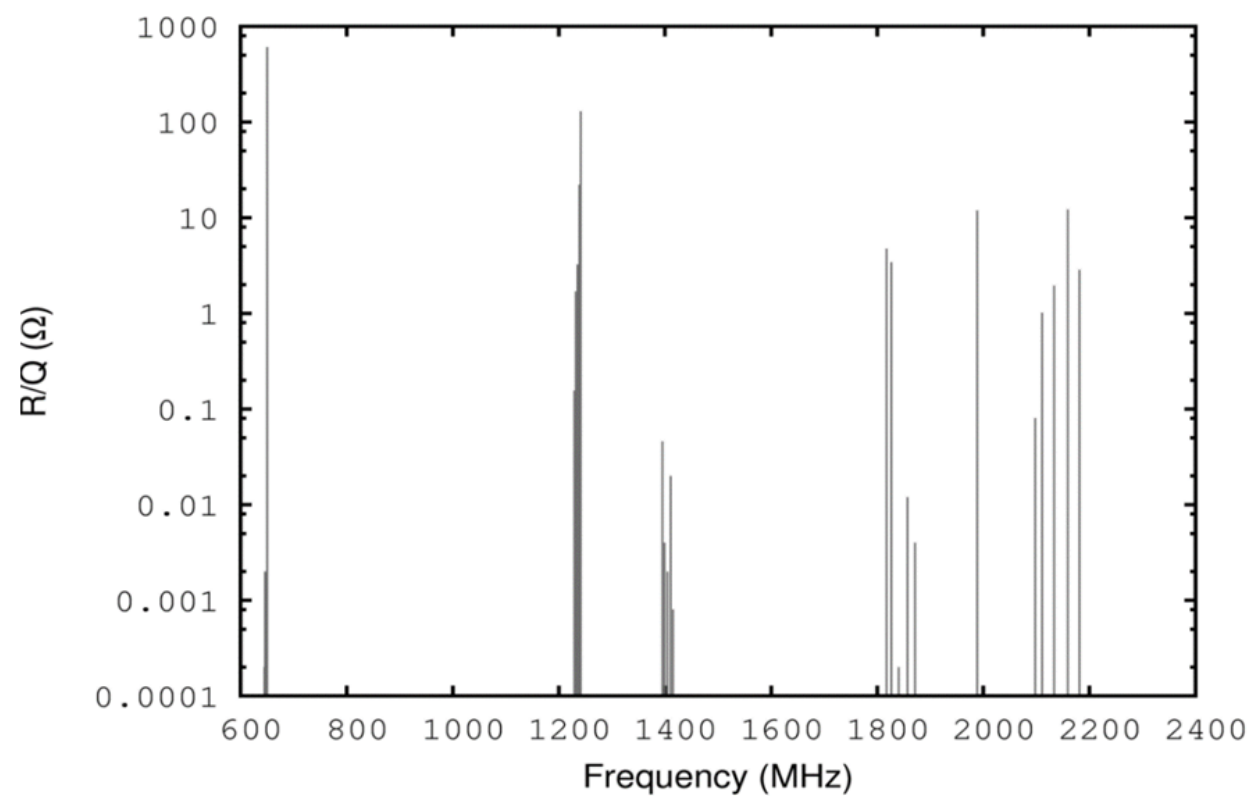

Figure 3.28: (R/Q) of the monopole HOMs in the HB650 cavities.

The amplitude of an excited monopole HOM, $U_{H O M}$, depends on the amplitude of the nearest beam spectrum line, $I$, the detuning $\delta f$, and the distance between the HOM frequency $f$ and the beam spectrum line frequency. It can be estimated for a high $Q$ resonance (assuming $\delta f / f \ll 1 / Q$ ) by the following:

$$
U_{\text {HOM }} \approx \frac{I(R / Q)}{4 \sqrt{2} \delta f / f} .
$$

If the high order mode is exactly at resonance, then

$$
U_{H O M}=\frac{1}{2} I(R / Q) Q_{L},
$$

where $Q_{L}$ is the loaded quality factor of the mode. The cryogenic losses depend on the square of the HOM amplitude:

$$
P_{\text {loss }} \approx \frac{U_{H O M}^{2}}{(R / Q) Q_{0}} .
$$

Requiring $P_{\text {loss }}$ to be much smaller than the sum of the static heat load and the cryogenic losses due to the accelerating mode $(20 \mathrm{~W})$, and assuming the intrinsic quality factor is $Q_{0}=5 \times 10^{9}$ one obtains the maximum allowable value of the monopole HOM loaded quality factor to be: $Q_{L} \ll 6 \times$ $10^{7}$.

Similarly, requiring that excitation of a monopole mode does not increase longitudinal emittance, $\varepsilon_{z} \gg U_{\text {HоM }} \sigma_{z} / c$, an estimation of the safe frequency detuning yields:

$$
\delta f \gg f \frac{I(R / Q) \sigma_{z}}{4 \sqrt{2} \varepsilon_{z} c} .
$$

Here $\sigma_{z}$ is the bunch length, and $c$ is the speed of light. The worst case is at the beginning of $\beta_{G}=0.92$ section, where the bunch length is maximum $\left(\sigma_{z} / c=7.7 \times 10^{-3} \mathrm{~ns}\right)$. Assuming that the second pass-band monopole HOM (1241 MHz and $R / Q=130 \mathrm{Ohm})$ is the nearest beam 
spectrum line $(I=1 \mathrm{~mA})$, and an emittance of $\varepsilon_{z}=1.5 \mathrm{keV} \mathrm{ns}$, one obtains the following estimate for frequency detuning: $\delta f \gg 140 \mathrm{~Hz}$.

A more accurate estimate of coherent HOM excitation in the Project $\mathrm{X}$ linac is performed using statistical analysis based on the expected spread of data for the HOM parameters (frequency, impedance and quality factor). Errors of cavity shape introduced in manufacturing are taken into account by allowing random variations of the cavity profile within $0.2 \mathrm{~mm}$ of ideal shape. In order to estimate the probability of cryogenic losses, and relative change of longitudinal emittance, $10^{5}$ random linacs were generated using predicted deviations of frequency, loaded quality factors and impedances of monopole HOMs. It was found that the probability to have losses above $0.1 \mathrm{~W}$ per cryomodule is extremely small: $10^{-4}$ for an average beam current of $1 \mathrm{~mA}$.

The beam structure, shown in Figure 3.26 consists of three main sub-components $(1 \mathrm{MHz}, 10$ $\mathrm{MHz}$ and $20 \mathrm{MHz}$ ). The phase of the voltage of an HOM excited by the resonance with one of the beam components is random with respect to two other components of the beam. In case of a high- $Q$ resonance such a HOM may introduce a significant energy variation and longitudinal emittance growth along the beam train. Results of statistical analysis show, that the probability of the emittance to double is $10^{-3}$ for the beam current of $1 \mathrm{~mA}$. Based on this analysis the conclusion is made that HOM couplers are not needed in $650 \mathrm{MHz}$ cavities. More details can be found in Ref. [75].

\subsubsection{The $325 \mathrm{MHz}$ and $650 \mathrm{MHz}$ Main Couplers.}

Main RF power couplers have to provide reliable operation of accelerator cavities at the following power levels: $17 \mathrm{~kW}$ at $325 \mathrm{MHz}$ and $64 \mathrm{~kW}$ at $650 \mathrm{MHz}$.

Criteria for coupler design are: reliability, minimizing production and operating costs. The coupler parameters chosen on the base of these requirements are presented in Table 3.8. The views of the couplers are presented in Figure 3.29.

Table 3.8: Parameters of 325 the MHz and 650 MHz Main Couplers.

\begin{tabular}{l|l|l}
\hline \hline Operating Frequency & $325 \mathrm{MHz}$ & $650 \mathrm{MHz}$ \\
\hline Output diameter & $3 ",, \mathrm{SS}$, not coated & $3,,, \mathrm{SS}$, copper coated \\
\hline Antenna diameter & $0.5^{\prime \prime}$, copper & $0.5 '$, copper \\
\hline Antenna cooling & Air & Air \\
\hline Window & Single, $\mathrm{Al}_{2} \mathrm{O}_{3}, 6 \mathrm{~mm}$ & Single, $\mathrm{Al}_{2} \mathrm{O}_{3}, 6 \mathrm{~mm}$ \\
\hline Input & $3-1 / 8^{\prime \prime}$ coaxial & Rectangular waveguide \\
\hline Multipactor suppression & $\mathrm{HV}$ bias & $\mathrm{HV}$ bias \\
\hline Cryo-load, $2 \mathrm{~K}, 0 \mathrm{~kW} / \mathrm{P}_{\max } *$ & $0.06 \mathrm{~W} / 0.5 \mathrm{~W}$ & $0.24 \mathrm{~W} / 0.45 \mathrm{~W}$ \\
\hline Cryo-load, $5 \mathrm{~K}, 0 \mathrm{~kW} / \mathrm{P}_{\max } *$ & $0.58 \mathrm{~W} / 2.8 \mathrm{~W}$ & $1.8 \mathrm{~W} / 2.7 \mathrm{~W}$ \\
\hline Cryo-load, $70 \mathrm{~K}, 0 \mathrm{~kW} / \mathrm{P}_{\max } *$ & $2.0 \mathrm{~W} / 5.4 \mathrm{~W}$ & $4.4 \mathrm{~W} / 6.0 \mathrm{~W}$ \\
\hline \hline
\end{tabular}

$* \mathrm{P}_{\max }=30 \mathrm{~kW}$ traveling wave for $325 \mathrm{MHz}, \mathrm{P}=120 \mathrm{~kW}$ traveling wave for $650 \mathrm{MW}$

To make coupler production more effective, an approach of maximum unification of parts was chosen during electromechanical design. Couplers for both frequencies should contain maximum number of common (shared) parts. 

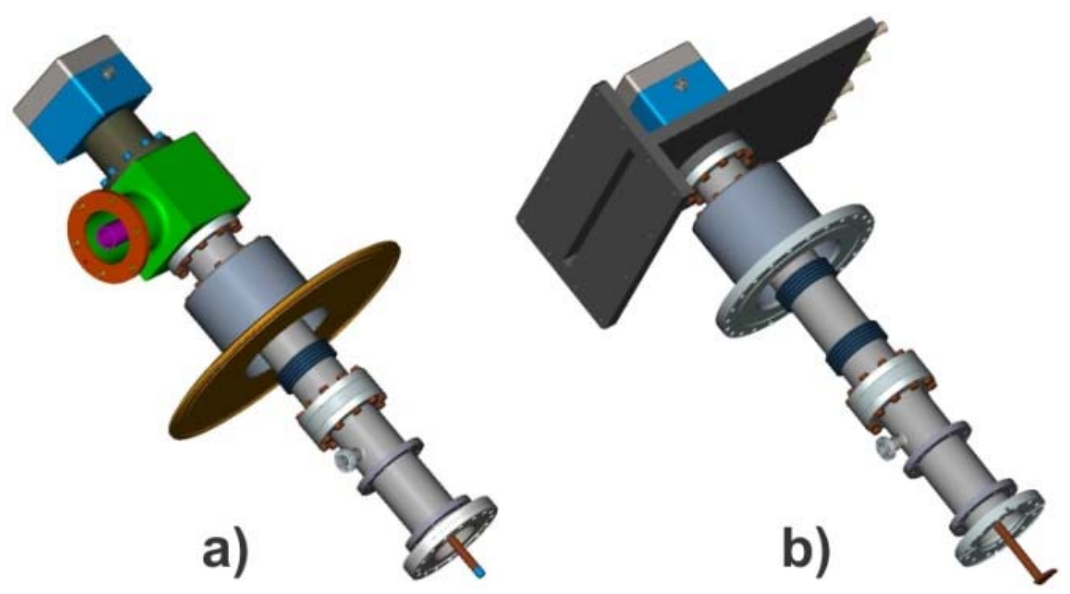

Figure 3.29: General views of a) $325 \mathrm{MHz}$ and b) $650 \mathrm{MHz}$ couplers.

Table 3.9: Design parameters of the SSR1 main coupler.

\begin{tabular}{l|l}
\hline \hline & Requirement \\
\hline CW Power & $30 \mathrm{~kW}$ \\
\hline Multipactor threshold & $25 \mathrm{~kW}$ (Trav. Wave) \\
\hline Passband & $50 \mathrm{MHz}$ \\
\hline Input impedance & $50 \Omega$ \\
\hline Output & $3{ }^{\prime} \times 0.5^{\prime}$, coaxial \\
\hline Output impedance & $105 \Omega$ \\
\hline
\end{tabular}

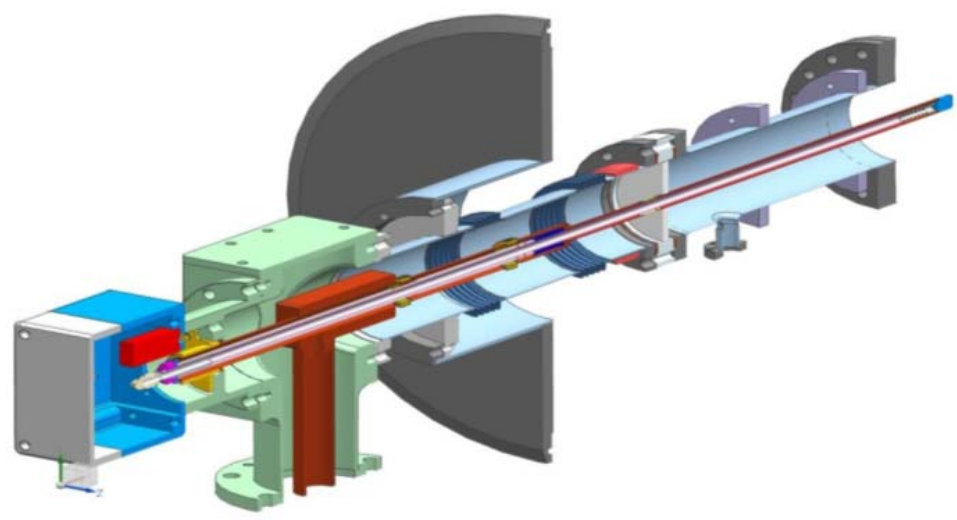

Figure 3.30: View of the SSR1 input coupler.

The coupler for $325 \mathrm{MHz}$ was designed, built and successfully tested. Its design is based on a $105 \Omega$ coaxial line. The coupler will be used for both SSR1 and SSR2 cryomodules. Its maximum power of $30 \mathrm{~kW} \mathrm{CW}$ is determined by requirements for an eventual upgrade of PIP-II to $5 \mathrm{~mA}$ average beam current [73]. The coupler contains a single warm ceramic window that provides separation of the warm and cold coupler sections. During cryomodule fabrication, the cold section can be installed on the cavity in the cleanroom prior to assembly of the string. The warm section can then be installed from outside the vacuum vessel during final assembly. The inner conductor is solid copper with phosphor bronze bellows to accommodate motion due to misalignment and 
thermal contraction. The cold end of the outer conductor is 316L-stainless steel. The warm end is copper with phosphor bronze bellows. Heat load estimates don't suggest a significant penalty for not copper plating the outer conductor. A forced-air cooling tube is inserted into the inner conductor after assembly that supplies air to cool the coupler tip. The coupler parameters are shown in Table 3.9. Figure 3.30 shows details on the coupler design.

\subsubsection{Measures Aimed at Reduction of RF Loss in Walls of SC Cavities}

Cryogenic loss in a cavity is determined by the R/Q value, $\mathrm{G}$-factor and surface resistance. The surface resistance in its turn is a sum of the residual resistance and the BCS resistance.

Through other sections of this document we use a conservative approach based on the Q-values already achieved in operating cryomodules. In this case, the BCS resistance as a function of the frequency and temperature may be estimated using the following formula,

$$
R_{B C S} \approx 2 \cdot 10^{-4} \frac{1}{T}\left(\frac{f}{1.5 \cdot 10^{9}}\right)^{2} e^{-17.67 / T},
$$

where the frequency, $f$, is measured in $\mathrm{Hz}$, and the temperature, $T$, in $\mathrm{K}$. For $650 \mathrm{MHz}$ and $2 \mathrm{~K}$ one obtains RBCS $\sim 2.7 \mathrm{n} \Omega$. Modern surface processing technology provides a residual resistance of $\sim 5$ $\mathrm{n} \Omega$ [27] resulting in the total resistance of $\sim 8 \mathrm{n} \Omega$. Assuming a medium field Q-slope at the peak field of $70 \mathrm{mT}$ of about $30 \%$, this yields a target for $\mathrm{Q}_{0}$ value of the $650 \mathrm{MHz}$ cavity of $\sim 2 \times 10^{10}$. This value was used above in Section 2.1.3.2.

However, there has been recent significant progress in improvement of quality factors of SRF cavities via two breakthroughs: cavity surface doping with nitrogen [77] and manipulation of trapped magnetic flux via cooling [78].

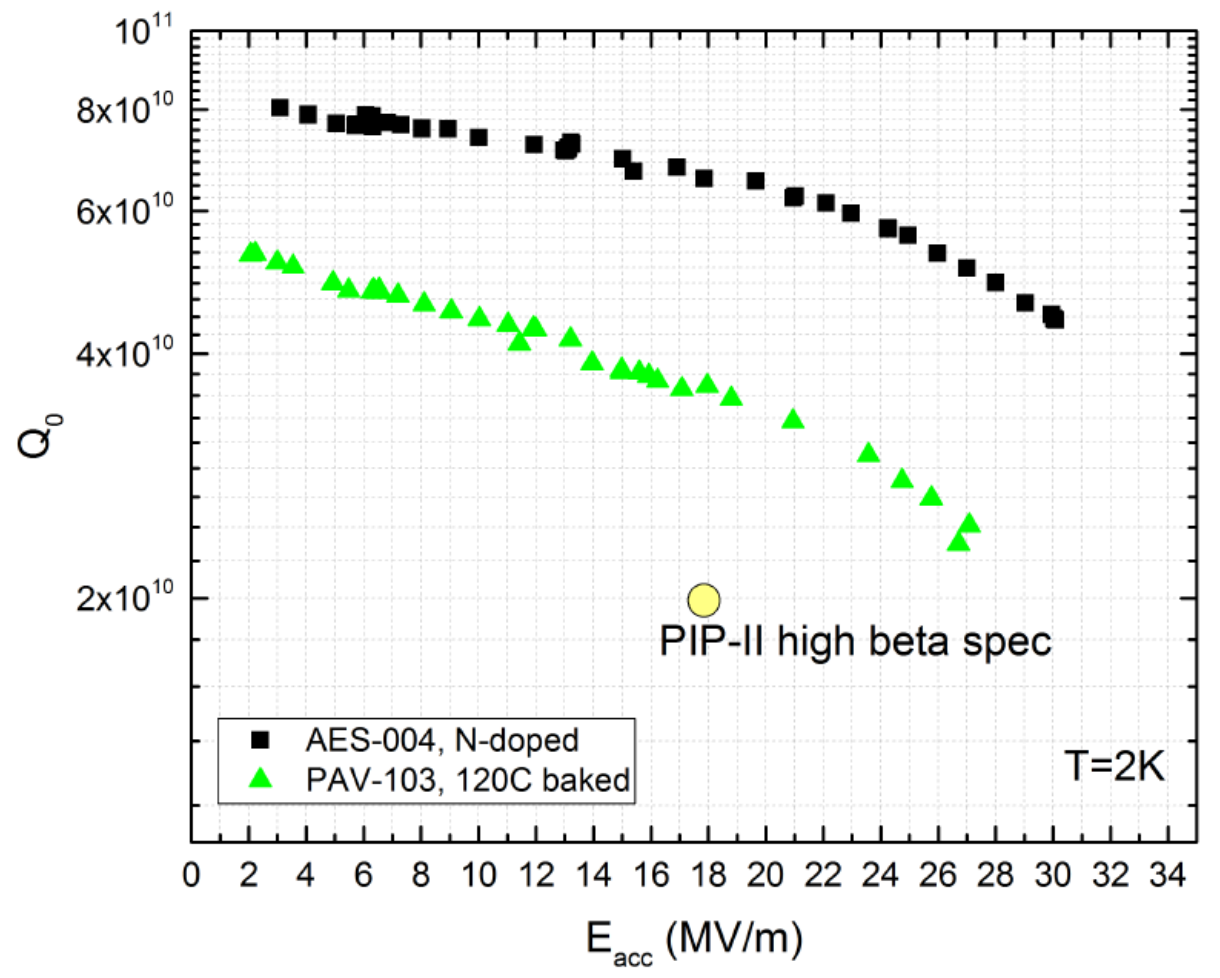

Figure 3.31: The dependence of $\mathrm{Q}_{0}$ on the accelerating voltage for the $650 \mathrm{MHz}, 120 \mathrm{C}$ baked cavity and the $\mathrm{N}$ doped cavity. The first one was manufactured by AES and another one by PAVAC. The measurements were carried out at $2 \mathrm{~K}$. 
The first finding has enabled lowering both BCS and residual resistance components for 1.3 $\mathrm{GHz}$ cavities systematically by a factor of more than two. More than twenty $1.3 \mathrm{GHz}$ cell cavities have been treated with $\mathrm{N}$ doping at Fermilab, Jlab and Cornell, achieving an average $\mathrm{Q} \sim 3.5 \cdot 10^{10}$ at $16 \mathrm{MV} / \mathrm{m}$ and $2 \mathrm{~K}$, which is three times higher than with standard $120 \mathrm{C}$ bake processing (ILC/XFEL recipe). Recently also several $650 \mathrm{MHz}$ cavities have been treated with standard 120C bake processing and $\mathrm{N}$ doping, showing outstanding results far exceeding the current specifications for PIP-2 of $2 \cdot 10^{10}$ at $2 \mathrm{~K}$ and $17 \mathrm{MV} / \mathrm{m}$, as shown in Figure 3.31 . For the $120 \mathrm{C}$ bake cavities the $\mathrm{Q}_{0}$ is $3.5 \cdot 10^{10}$ at $2 \mathrm{~K}$ and $16 \mathrm{MV} / \mathrm{m}$. For the $\mathrm{N}$ doped ones $\mathrm{Q}_{0}$ have reached $7 \cdot 10^{10}$ at $2 \mathrm{~K}$ and $17 \mathrm{MV} / \mathrm{m}$, exceeding by a factor of more than three the current PIP-2 specifications. With such quality factors and the cryo-plant capacity considered in Sections 2.1.3.2 and 3.4 the cryo duty-factor could be increased by about three times from $\sim 5 \%$ to $\sim 15 \%$. That corresponds to an order of magnitude increase of the beam duty-factor from $\sim 1 \%$ to $\sim 11 \%$. That enables experiments with high duty factors even with the present cryo-plant capacity.

Slow versus fast cooling has been demonstrated to significantly deteriorate performance of 1.3 $\mathrm{GHz}$ cavities due to poor flux expulsion efficiency. Recently the same experiment has been performed for $650 \mathrm{MHz}$ cavities and results showed very little degradation with slow cooling versus fast cooling, as shown in Figure 3.32, hinting to a likely weaker losses dependence of trapped flux due to the lower RF frequency. This is promising for full realization of these high quality factors in cryomodule, where magnetic flux manipulation becomes more challenging.

Further studies are aimed to assure that the results achieved in a vertical test can be obtained in an operating cryomodules.

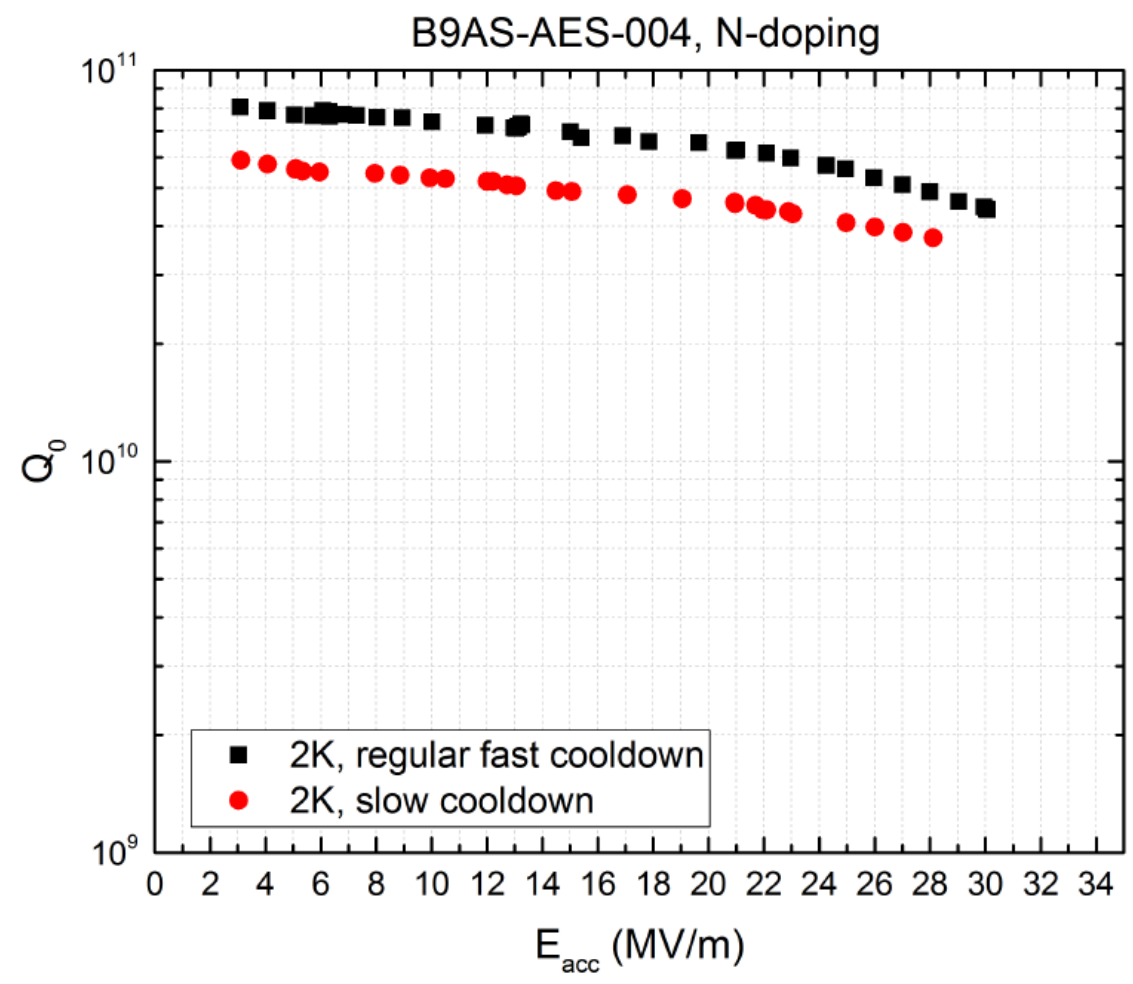

Figure 3.32: The dependence of $\mathrm{Q}_{0}$ on the accelerating voltage for the $\mathrm{N}$ doped $650 \mathrm{MHz}$ cavity for the fast and slow cool downs. 


\subsubsection{RF Power and Low Level RF}

\subsubsection{RF Power}

There are $120 \mathrm{RF}$ systems included in the linac. With the exception of the RFQ they are deployed as one amplifier per cavity. Parameters of the RF amplifiers are presented in Table 3.10. In comparison to the power requirements presented in Table 2.11 the RF amplifier powers were rounded to about $\sim 10 \%$ larger values to get an allowance for operation with some cavities operating at reduced accelerating gradient. All RF systems will utilize continuous wave (CW) amplifiers, although SSR1, SSR2, LB650 and HB650 will operate in the pulsed regime to reduce $\mathrm{RF}$ cryogenic power. They will be used in $\mathrm{CW}$ mode later when the SC Linac will be upgraded to run for muon experiments.

Table 3.10. Parameters of RF amplifiers

\begin{tabular}{|l|c|c|c|c|c|}
\hline & $\begin{array}{c}\text { Frequency } \\
\text { (MHz) }\end{array}$ & $\begin{array}{c}\text { Number } \\
\text { of RF } \\
\text { cavities }\end{array}$ & $\begin{array}{c}\text { Number of RF } \\
\text { amplifiers per } \\
\text { cavity }\end{array}$ & $\begin{array}{c}\text { Regime } \\
\text { of } \\
\text { operation }\end{array}$ & $\begin{array}{c}\text { RF } \\
\text { amplifier } \\
\text { power (kW) }\end{array}$ \\
\hline RFQ & 162.5 & 1 & 2 & $\mathrm{CW}$ & 75 \\
\hline MEBT Bunching cavities & 162.5 & 3 & 1 & $\mathrm{CW}$ & 3 \\
\hline First HWR cavity & 162.5 & 1 & 1 & $\mathrm{CW}$ & 3 \\
\hline Other HWR cavities & 162.5 & 7 & 1 & $\mathrm{CW}$ & 7 \\
\hline SSR1 & 325 & 16 & 1 & Pulsed & 7 \\
\hline SSR2 & 325 & 35 & 1 & Pulsed & 20 \\
\hline LB650 & 650 & 33 & 1 & Pulsed & 40 \\
\hline HB650 & 650 & 24 & 1 & Pulsed & 70 \\
\hline
\end{tabular}

The RFQ has two input ports and is driven by two $75 \mathrm{~kW} \mathrm{CW}$ solid-state amplifiers. Three room temperature buncher cavities and one cryomodule containing eight superconducting HWRs operate at $162.5 \mathrm{MHz}$. They will have one solid-state amplifier each operating at power levels of 3 to 7 $\mathrm{kW}$. The first HWR cavity operates at about half of the accelerating voltage and therefore uses smaller power. Two SSR1 cryomodules operating at $325 \mathrm{MHz}$ will be populated with eight cavities each and powered by $7 \mathrm{~kW}$ solid-state amplifiers. Seven SSR2 cryomodules with 5 cavities each will be powered by $20 \mathrm{~kW}$ solid-state amplifiers. Eleven LB650 cryomodules with 33 cavities and four HB650 cryomodules with 24 cavities will each be powered by IOT amplifiers. It is possible that a solid-state or injection locked magnetron will be used at $650 \mathrm{MHz}$ as R\&D for those technologies mature.

The RF distribution system for the $\mathrm{CW}$ linac will utilize rigid coax commensurate with system power levels, 6-1/8", 3-1/8", or 1-5/8" EIA flanged sections. The final connection to the cryomodules will utilize a section of flexible transmission line to minimize connector location tolerances. Each RF system will have a circulator and a load to isolate the cavity from the power amplifier. This level of protection is essential in SRF systems due to the full power reflection from the cavity in the absence of beam. Cavity and drive sample signals will be provided to the LLRF for vector regulation and frequency control of the cavities. All of the RF amplifiers will be water cooled to minimize the heat load to the building HVAC system.

While each amplifier has built in protection which includes, water flow, water temperature, 
pressure differential, and reflected power monitoring; a global interlock and hardware protection system must be designed for all RF systems. This will include water flow to loads and circulators, spark detection on cavity couplers, and RF leakage detection.

The low level RF (LLRF) system will provide a drive signal on the order of 0 to $+10 \mathrm{dBm}$ for each RF power source. The amplifier(s) will provide sample signals of the pre-driver and final outputs. All amplifiers will be self-contained units complete with integral power supplies, protection circuits, and control interface.

\subsubsection{Active Suppression of Microphonics and Lorentz Force Detuning}

High accelerating gradient and comparatively small beam current result in high values of loaded quality factors, and, consequently, narrow bandwidth and high sensitivity to microphonics (see Chapter 2.1.33). It is difficult to accurately predict uncompensated detuning levels because detuning can be driven by such a variety of different factors. Crude estimates of the range of expected levels can be made by examining the pressure regulation, cavity sensitivity and vibration levels measured in existing machines.

Pressures in large cryogenic systems can be easily regulated to $1 \%$ or better [31]. SNS and JLab are able to maintain steady state pressures to within $100 \mathrm{uBar}$ and $25 \mathrm{uBar}$ respectively but both can experience occasional much larger transients. With careful design cavity pressure sensitivity $(d f / d P)$ can be reduced to a few Hz/Torr. The sensitivity of the Quarter Wave Resonator and Half Wave Resonators of ISAC-II machine at TRIMF was $\sim 3 \mathrm{~Hz} /$ torr and $<1 \mathrm{~Hz} /$ Torr respectively. The sensitivity of the most recent SSR1 prototype developed at Fermilab is $4 \mathrm{~Hz} /$ Torr. This prototype was specifically designed to minimize $d f / d P$. An earlier prototype which was not designed with the explicit goal of minimizing $d f / d P$ exhibited a sensitivity of $150 \mathrm{~Hz} /$ Torr.

Estimates for the expected range of detuning levels due to mechanical vibrations can be extracted from measurements of rms pulse-to-pulse variations in the resonance frequencies of the $1.3 \mathrm{GHz}$ elliptical cavities in the two cryomodules, CM1 and CM2, tested in NML in at Fermilab. Detuning levels ranged between $8 \mathrm{~Hz}$ for cavities at either end of the cryomodules to $2 \mathrm{~Hz}$ for cavities at the center of the cryomodules. The larger values measured for the end cavities were attributed to vibrations caused by nearby vacuum pumps. The levels measured in the NML cryomodules represent only detuning due to mechanical vibrations. The CM1 and CM2 cavities have pressure sensitivities of approximately $50 \mathrm{~Hz} /$ Torr, but the NML detuning control system adjusts the piezo bias voltage on a pulse-by-pulse basis to compensate for variations in the helium bath pressure.

Detuning due to the Lorentz force may vary between cavity types depending on the design. The resonance frequency of the SSR1 prototype varies by about $0.5 \mathrm{kHz}$ as the cavity is ramped to full gradient. Table 3.11 collects the ranges of detuning expected from each individual source and gives a range of peak uncompensated detuning expected from all sources combined, while Table 2.12 lists the parameters relevant to detuning compensation for each PIP-II cavity type.

A comparison of the narrow matched bandwidths planned for the PIP-II cavities to the expected range of uncompensated detuning emphasizes the importance of exploiting all possible passive compensation measures. Cavities must be designed to minimize sensitivity to pressure; the cryogenic system must be designed to minimize pressure variations; and the cryomodule and other systems of the SC Linac must be designed to minimize vibrations transmitted to the cavities.

Even if all passive measures are fully exploited some form of active detuning compensation will almost certainly be required even for future $\mathrm{CW}$ operation of SC Linac. It is absolutely required for the pulsed operation of PIP-II. 
Table 3.11: Expected ranges of uncompensated detuning from random sources for the PIP-II cavities

\begin{tabular}{|c|c|c|c|}
\hline \multicolumn{4}{|c|}{ Pressure Related Detuning } \\
\hline Source & Units & Lower & Upper \\
\hline Peak pressure variation & mbar & 0.02 & 0.3 \\
\hline Mean pressure sensitivity & $\mathrm{Hz} / \mathrm{mbar}$ & 5 & 150 \\
\hline Peak pressure sensitivity variation & $\mathrm{Hz} / \mathrm{mBar}$ & 5 & 5 \\
\hline Peak pressure related detuning & $\mathrm{Hz}$ & 0.2 & 45 \\
\hline
\end{tabular}

\begin{tabular}{|c|c|c|c|}
\hline \multicolumn{4}{|c|}{ Vibration Related Detuning } \\
\hline Source & Units & Lower & Upper \\
\hline RMS detuning due to mechanical vibrations & $\mathrm{Hz}$ & 2 & 8 \\
\hline Peak (6 Sigma) detuning due to mechanical vibrations & $\mathbf{H z}$ & 12 & 48 \\
\hline
\end{tabular}

\begin{tabular}{|l|c|c|c|}
\hline \multicolumn{4}{|c|}{ Lorentz Force Related Detuning } \\
\hline Source & Units & Lower & Upper \\
\hline Peak detuning due to the Lorentz Force & $\mathbf{H z}$ & $\mathbf{1}$ & $\mathbf{5 0}$ \\
\hline
\end{tabular}

\begin{tabular}{|c|c|c|c|}
\hline \multicolumn{4}{|c|}{ Total Uncompensated Detuning } \\
\hline Source & Units & Lower & Upper \\
\hline Peak uncompensated detuning $(\mathrm{Hz})$ & $\mathbf{H z}$ & 13 & 143 \\
\hline
\end{tabular}

\section{Detuning Compensation for PIP-II}

Minimizing detuning in the PIP-II cavities will require a three-pronged approach:

- Fully exploiting all passive compensation measures during cavity and cryomodule design,

- Development and validation of active detuning compensation algorithms, and

- Detuning Control System engineering.

All of the PIP-II cavities types will be designed to minimize $d f / d P$. The pressure sensitivity of the second SSR 1 prototype was designed to minimize $d f / d P$ at $5 \mathrm{~Hz} /$ Torr compared to $150 \mathrm{~Hz} /$ Torr for the first prototype. Prototypes of the other four PIP-II cavity types have not yet been constructed or tested. Steps that may be taken to minimize Lorentz force detuning and mechanical vibrations are under investigation but no design goals have been set as yet.

The first step towards implementing an active detuning compensation system for the PIP-II cavities is the development, demonstration and validation of an appropriate set of algorithms using single cavities and prototype cryomodules. Once the performance of these algorithms has been satisfactorily demonstrated, they can be integrated into the Low Level RF control system.

While considerable progress has been made in the active stabilization of SRF cavity resonance frequencies using piezo actuator over the last decade, the field is still in its infancy. DESY pioneered the use of piezo actuators to compensate for Lorentz force induced detuning of the SRF cavities. Studies in the HoBiCaT test stand at BESSY, Berlin, showed that the resonance frequency of $1.3 \mathrm{GHz}$ elliptical $\mathrm{CW}$ cavities could be actively stabilized to better than $1 \mathrm{~Hz} \mathrm{rms}$. An adaptive feed-forward algorithm developed at Fermilab has been used to successfully stabilize the resonance for both CW and pulsed SRF cavities. While these techniques were able to control detuning in individual cavities over the duration of each respective test, it has yet to be demonstrated that any 
of those techniques can routinely stabilize the resonance frequency for every cavity in a CW or pulsed machine with narrow-bandwidth SRF cavities to the required level over the entire planned machine lifetime. The factors that drive the performance of active stabilization are still not well understood. Until they are, it will be difficult if not impossible to engineer a system capable of meeting the detuning control requirements for such a linac.

\section{Optimal Control and System Engineering}

In sharp contrast to the ad-hoc techniques employed to date, optimal control offers a welldefined systematic approach to the problem of combined electro-mechanical control of SRF cavities. The Lorentz force couples the electromagnetic and mechanical states of the cavities. Knowledge of one should aid in the compensation of the other but the techniques employed to date treat resonance stabilization independently of the control of the cavity gradient and phase. Optimal control techniques were pioneered by Richard Kalman of Stanford in the 1950s. Since then they have found wide use in a variety of areas of biology, economics, ecology, engineering, finance, management, and medicine but only limited use in accelerator control systems despite the performance improvements they could bring.

The first step in implementing an optimal SRF cavity control system involves characterizing the electro-mechanical response of each individual cavity via a series of stimulus-response measurements including detuning due to piezo response and detuning response to reactive power modulation. Models of the response suitable for use in subsequent steps can be extracted using the minimal state-space realization (MSSR) system-identification algorithm of Kalman and Ho.

The second step involves estimating the cavity electromechanical state at each point in time from real-time measurements of the cavity base-band RF signals. At each time step the Kalman filter determines optimal estimates of the cavity electro-mechanical state by minimizing a quadratic cost function that depends on the measured values of the cavity RF baseband signals, piezo actuator voltage and current, the previous state estimate, the covariance of the signal noise, the covariance of the previous state estimate, and the a-priori cavity response model determined in the first step.

In the final step a Linear Quadratic Gaussian Regulator (LQGR) minimizes at each point in time a cost function similar to that used by the Kalman filter to determine the combination RF and piezo drive signals most likely to keep the cavity gradient, phase and detuning at their target values.

These steps provide a well-grounded chain of deterministic algorithms that can be used to automatically characterize, model and optimally control detuning and field stability of any superconducting cavity or chain of cavities. Steady-state versions of the Kalman filter and LQGR can be implemented in real-time using commercially available FPGA signal processing boards.

The two SSR1 prototype cavities have been used to study microphonics compensation during tests in the STC test stand in the Meson Detector Building (MDB). Tests in 2011 using the first SSR1 prototype showed it was possible to stabilize the resonance frequency to less than $0.5 \mathrm{~Hz}$ over a period of 20 minutes with a quite simple algorithm. Further studies, following quality factor measurements for the second prototype, showed that it was possible to stabilize the resonance frequency of even very narrow band-width cavities for extended periods. Studies in the STC continue with the aim of developing and demonstrating the performance of a set of algorithms appropriate for active compensation of the PIP-II cavities.

Once a well-defined set of detuning compensation algorithms have been developed and their performance has been satisfactorily demonstrated using single cavities and the prototype cryomodules, the algorithms will be integrated into the Low Level RF control system. While the 
development stage will focus on meeting the required performance goals, the system engineering stage will focus on the large scale deployment of those algorithms. Reliable operation of the PIP-II machine will require an efficient and robust implementation capable of compensating detuning for every cavity for the lifetime of the accelerator.

\subsubsection{Low Level RF}

The Low Level RF system encompasses the programming and regulation of the cavity field amplitude and phase as required by the longitudinal beam dynamics in the machine. It also controls or interfaces to the ancillary equipment that is involved in the generation of RF. Hardware and software modules include Cavity Field Controller, Resonance Frequency Controller, Master Oscillator, Phase Reference Line, LO distribution, Transfer Synchronization to Booster, Beam Chopper Waveform Generator, and the interface to interlocks, timing systems and the control system (see Figure 3.33). The LLRF will also be involved in longitudinal phase space painting into the Booster.

The Linac is constructed with accelerator sections with the following frequencies; $162.5 \mathrm{MHz}$, $325 \mathrm{MHz}$ and $650 \mathrm{MHz}$. Additionally $1300 \mathrm{MHz}$ is provided for instrumentation and local clock generation. The basic configuration is that one RF amplifier will drive one cavity with the exception that two amplifiers will drive the RFQ. There is a mixture of warm copper cavities (the RFQ and buncher cavities) and 5 types of SRF cavities. There is also a mixture of CW systems with the HWR and SSR1 cavities and pulsed systems for the rest of the linac.

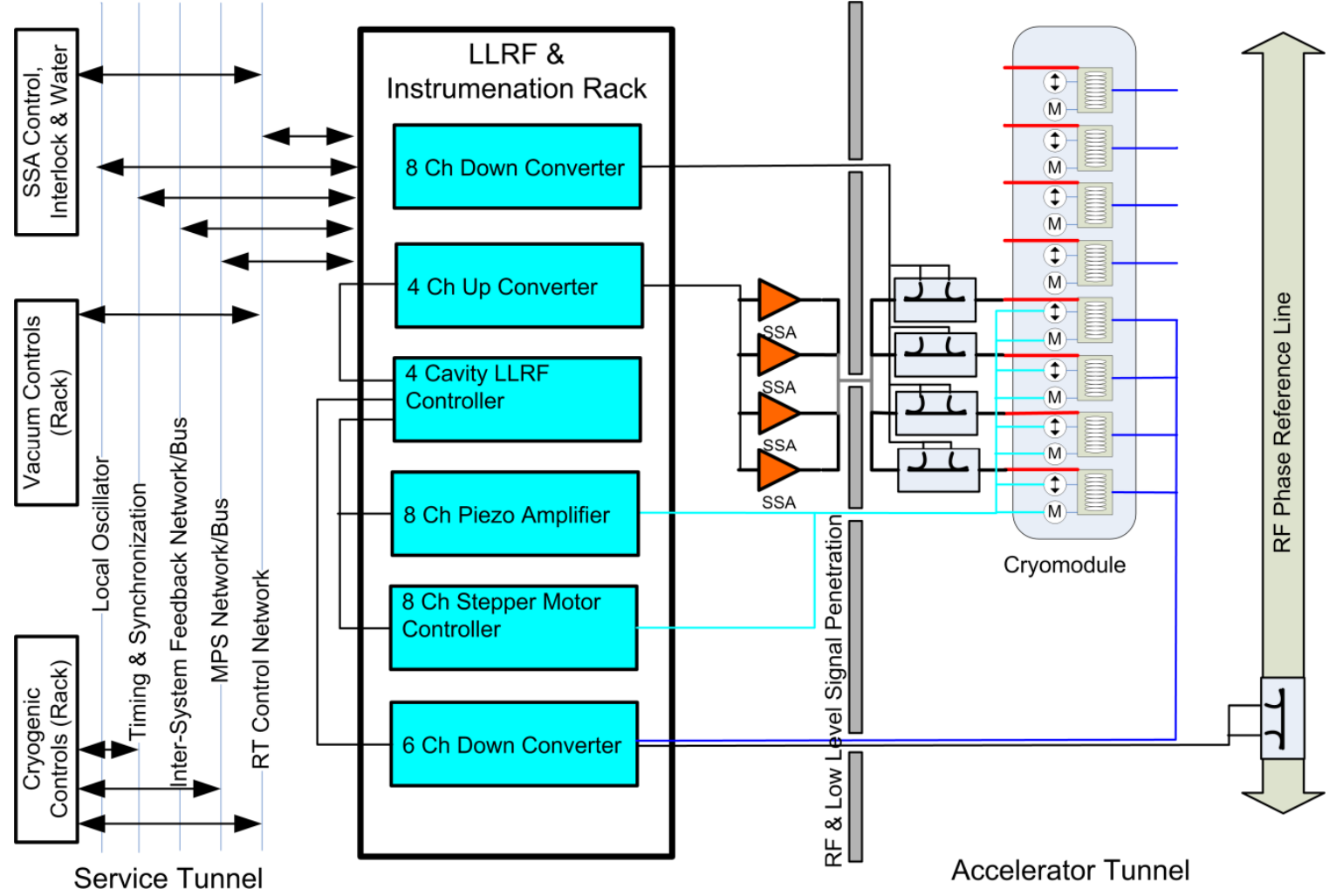

Figure 3.33: Four cavity RF system including LLRF rack.

\section{LLRF Regulation Strategy}

The requirements for the linac beam energy stabilization are determined by the Booster RF bucket height and, requirements related to the static longitudinal painting in the course of multi- 
turn beam injection (see Section 2.3.2.3) which result in the linac rms energy stability being better or about $0.01 \%$. The strategy to reach this extremely tight goal is based on a two-step energy stabilization. First, the voltage of each individual cavity is stabilized to $0.1 \%$ and 0.1 degree. Second, the beam based feedback, which uses a few last linac cavities, stabilizes the linac energy to $0.01 \%$. To achieve that, the beam-based energy feedback has to be sufficiently fast. For given cavity bandwidths it requires that the correcting signal has to be back to the linac cavities with less than 4 microsecond delay. The beam energy and phase measurements are used in two ways, first to make real time corrections using select cavities and, secondly, to provide input to the learning feedforward system. The learning feed-forward system greatly reduces the RF stability requirements for time periods greater than $1 \mathrm{~s}$ by correcting for cable delays and electronic system drifts.

The same regulation accuracy of about $0.01 \% \mathrm{rms}$ is required for the Booster magnetic field at the injection. It can be achieved with the scope extension of the new Booster cogging system which is presently under commissioning. In particular, the feedback system can be based on the magnetic field measurement in the Booster reference magnet with subsequent average magnetic field correction by Booster dipole correctors - similar to the new cogging system.

The beam current, cavity field gradients, Lorentz Force Detuning (LFD) and worst-case microphonics determine the loaded cavity Qs, bandwidths and RF power requirements (see Section 2.1.3.3). Precision corrections of the LFD and microphonic disturbances through Resonance Frequency Control, are required for gradient regulation without exceeding the available RF power overhead. Resonance control to meet these requirements requires a large coordinated engineering effort including both mechanical and electrical designs. Resonance control is covered below in more detail.

\section{Phase Reference Generation and Distribution}

The phase reference system starts at the Linac frontend with a $162.5 \mathrm{MHz}$ Master Oscillator (see Figure 3.34). The $162.5 \mathrm{MHz}$ Master Oscillator is inside a temperature controller chassis and generates the $182.8 \mathrm{MHz}$ local oscillator signals for the LLRF up-converters and down-converters for the $162.5 \mathrm{MHz}$ linac section. The $162.5 \mathrm{MHz}$ is driven into a closed loop phase averaging reference line that is located alongside the accelerating structure. The reference line is tapped providing signal to cables run alongside the cavity probe cables providing first order cable temperature compensation. This first reference line provides signal for the main timing system, instrumentation and the beam chopper controller.

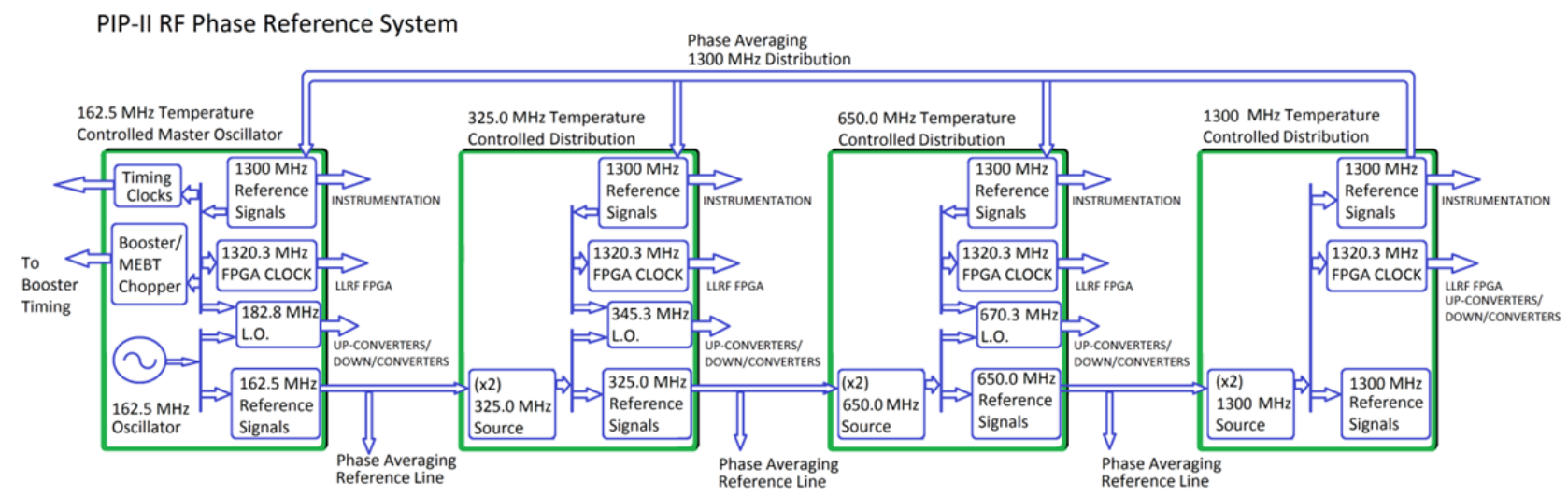

Figure 3.34: Master Oscillator and Multi-frequency Phase Reference Lines

The $162.5 \mathrm{MHz}$ phase reference line also provides signal to the $325 \mathrm{MHz}$ Slave Oscillator that 
tightly tracks the $162.5 \mathrm{MHz}$ phase. The $325 \mathrm{MHz}$ oscillator generates the $345.3 \mathrm{MHz}$ local oscillator signals in the same fashion as is done in the $162.5 \mathrm{MHz}$ master oscillator. A $325 \mathrm{MHz}$ phase averaging reference line is setup in the same fashion as the $162.5 \mathrm{MHz}$ reference scheme. This chain of frequency multiplication is repeated for the $650 \mathrm{MHz}$ sector and finally the 1300 $\mathrm{MHz}$ sector. The $1300 \mathrm{MHz}$ master oscillator also serves as the source for a phase averaging reference line that travels back down to the lower frequency sectors, providing $1300 \mathrm{MHz}$ taps for instrumentation, and it will provide for a single $1320.3 \mathrm{MHz}$ clock for LLRF digital systems distributed through the Linac.

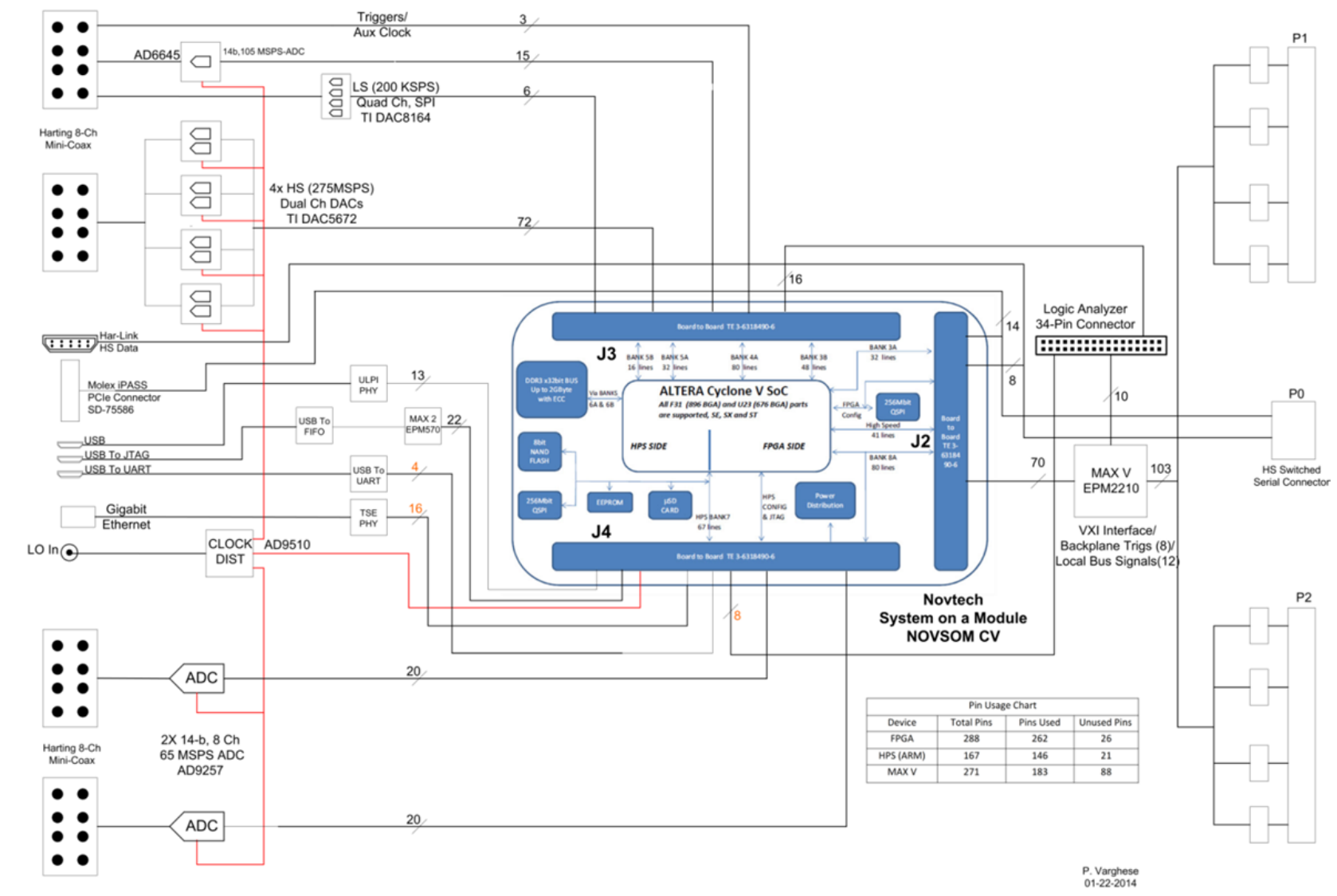

Figure 3.35: LLRF 4 Station Field Controller Module

\section{LLRF Control Rack}

The LLRF is organized in a group of up to four cavities serviced by one rack of electronics as shown in Figure 3.35. It includes control of the cavity voltage and cavity resonance and has the interface to the computer controls, timing and fast feedback systems. The group of four cavities allows for an economy of scale in the hardware design while keeping cable runs as short as possible. The signal path is kept as direct as possible with cables from the accelerator tunnel brought directly to precision receivers that down-convert the RF signals to a standard intermediate frequency (IF) that is common to all RF systems. The IF is digitized and then digitally processed for all control algorithms within Field Programmable Gate Arrays (FPGA). These algorithms generate the RF drive provided to the power amplifiers and the piezo actuator drive for cavity resonance control. Noise levels of lower than $-150 \mathrm{dBc} / \mathrm{Hz}$ and closed loop bandwidths of $\sim 50 \mathrm{kHz}$ are required for precision regulation. The controller is designed to support both $\mathrm{CW}$ and pulsed operation. It is expected that each cavity will be operated CW during some part of commissioning 
and tests. CW operation with low cavity bandwidths requires startup in a self-excited loop with a transition to a generator driven loop to align with the beam phase. Pure pulsed operation requires a complex phase trajectory program to fill the cavity with energy. A prototype system is under development for PIXIE. It includes the LLRF four Station Field Controller Module shown in Figure 3.35. The FPGA, CPU, memory and other interface components are located on a replaceable System on Module (SOM) allowing an easy upgrade path in the future of these rapidly advancing components. The LLRF system provides both RF waveforms and sampled values to the control system that are calibrated and highly linear. These best represent the cavity field and directional RF signals and will be used for all data analysis.

\section{Beam Chopper Waveform Generator and Booster Injection}

LLRF will also generate the waveforms needed for the beam chopper ${ }^{7}$ and the $44.705 \mathrm{MHz} \mathrm{RF}$ signal for the Booster to lock to during the 4 millisecond injection period. The waveforms require complex pre-distortion for the chopper amplifiers which is better implemented with the entire waveforms calculated and played out from tables. There are several advantages to waveform tables: repeatability from pulse to pulse, local storage of beam waveforms in LLRF and instrumentation systems, and learning in the generator and LLRF based on beam loss patterns. A multi-channel 4 GSPS arbitrary waveform generator is specified for this purpose.

\footnotetext{
${ }^{7}$ The beam chopper removes bunches on the boundary of RF buckets and forms a 3 bunch long extraction gap.
} 


\subsection{Booster}

\subsubsection{Radiation Shielding of the Booster Injection Absorber}

The geometry model used for the shielding calculations with the MARS15 code [79-81] for the injection absorber in the tunnel is shown in Figures. 3.36 and 3.37. The absorber consists of a $2^{\prime \prime} \times 2 " \times 12^{\prime \prime}$ tungsten core surrounded with 6 " of iron located on a concrete pedestal.

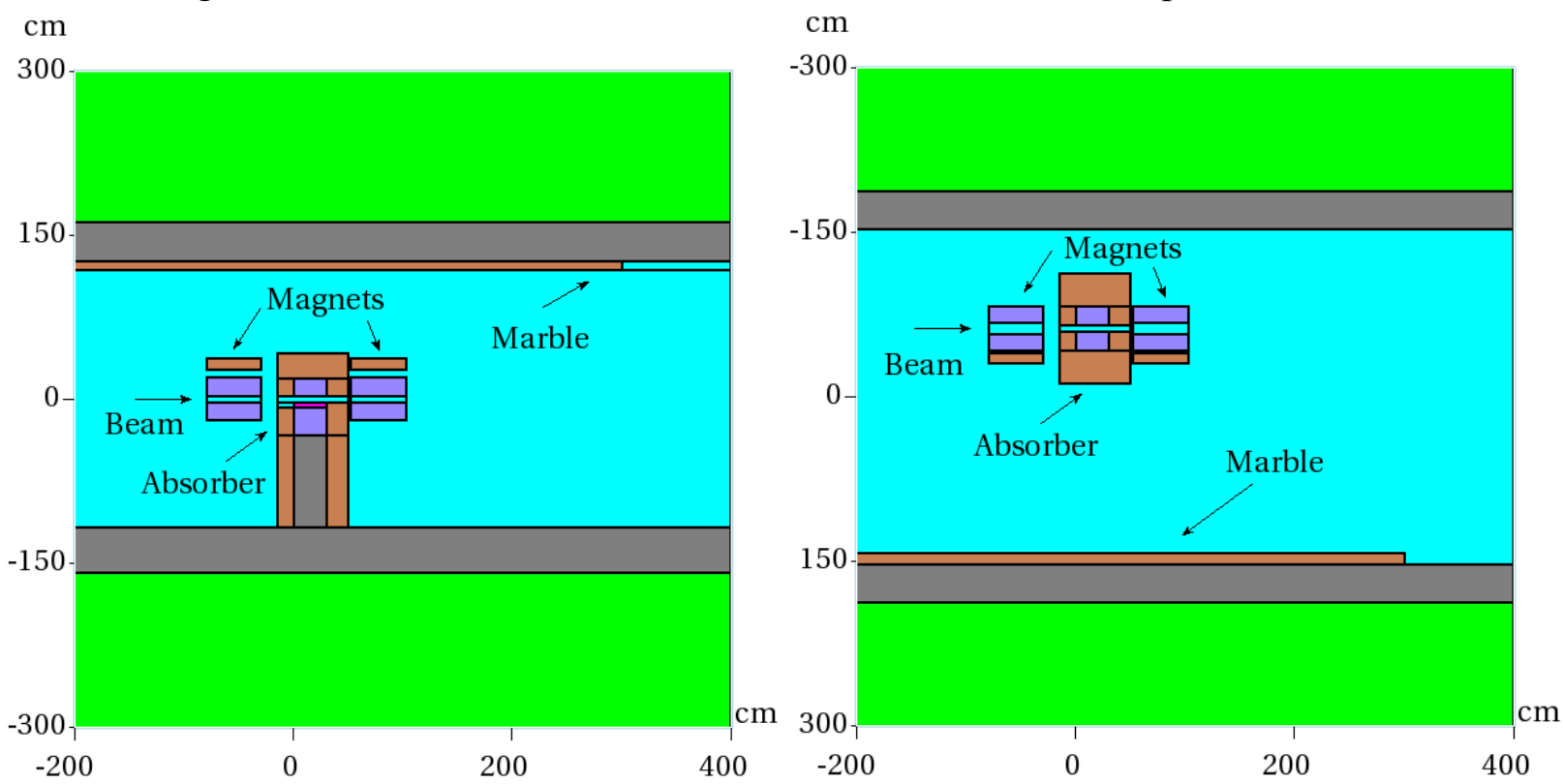

Figure 3.36: Elevation and plan view (left and right, respectively) of the MARS15 model of the absorber.

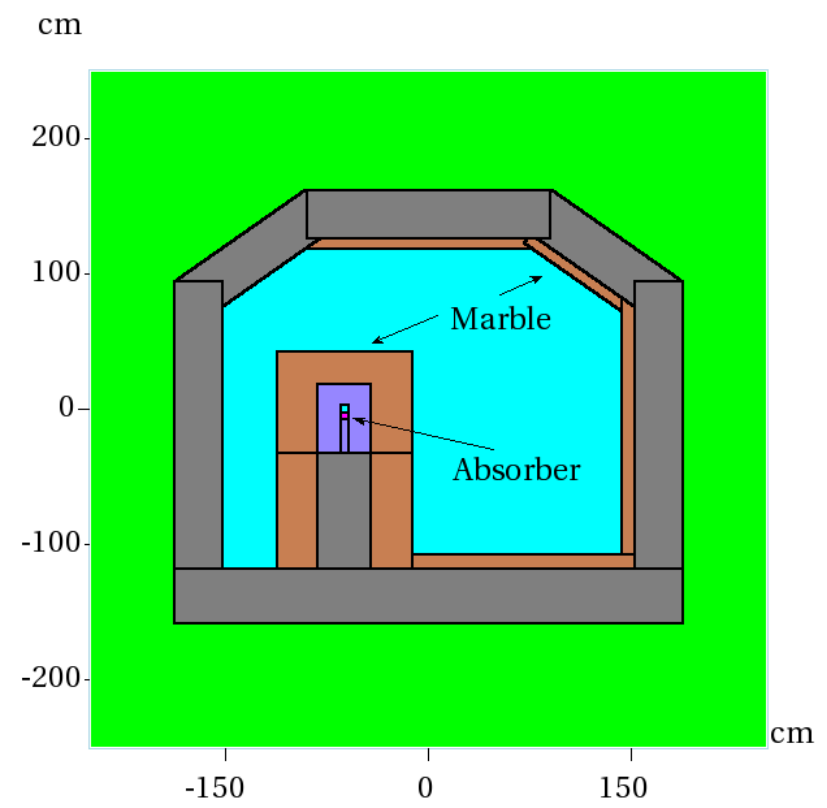

Figure 3.37. Cross section of the MARS15 model of the absorber in the tunnel.

In order to reduce residual activation of the absorber, the absorber core is surrounded with several thick layers of marble. The model also includes magnets upstream and downstream of the 
absorber as well as layers of marble (4" thick and 200" in length) on the tunnel floor, walls and ceiling mostly on the aisle side where the residual dose should be as low as possible. At the same time, comparison of the calculated residual dose on the wall side and aisle side reveals the high efficiency of the marble in terms of residual dose reduction. This model is a result of several iterations performed in order to reach acceptably low levels of surface water activation, residual activation of the absorber itself, tunnel, and magnets both upstream and downstream, as well as absorbed dose in the magnets. A comparison of the calculated residual dose on the wall side and isle side shown below reveals high efficiency of marble in terms of residual dose reduction.

The calculated star density distribution around the absorber has the peak value of about $7.66 \times 10^{-9} \mathrm{~cm}^{-3} \mathrm{p}^{-1}$ and is shown in Figure 3.38. According to the Fermilab concentration model, it means that the activated surface water should be removed with sump pumps approximately once a year.
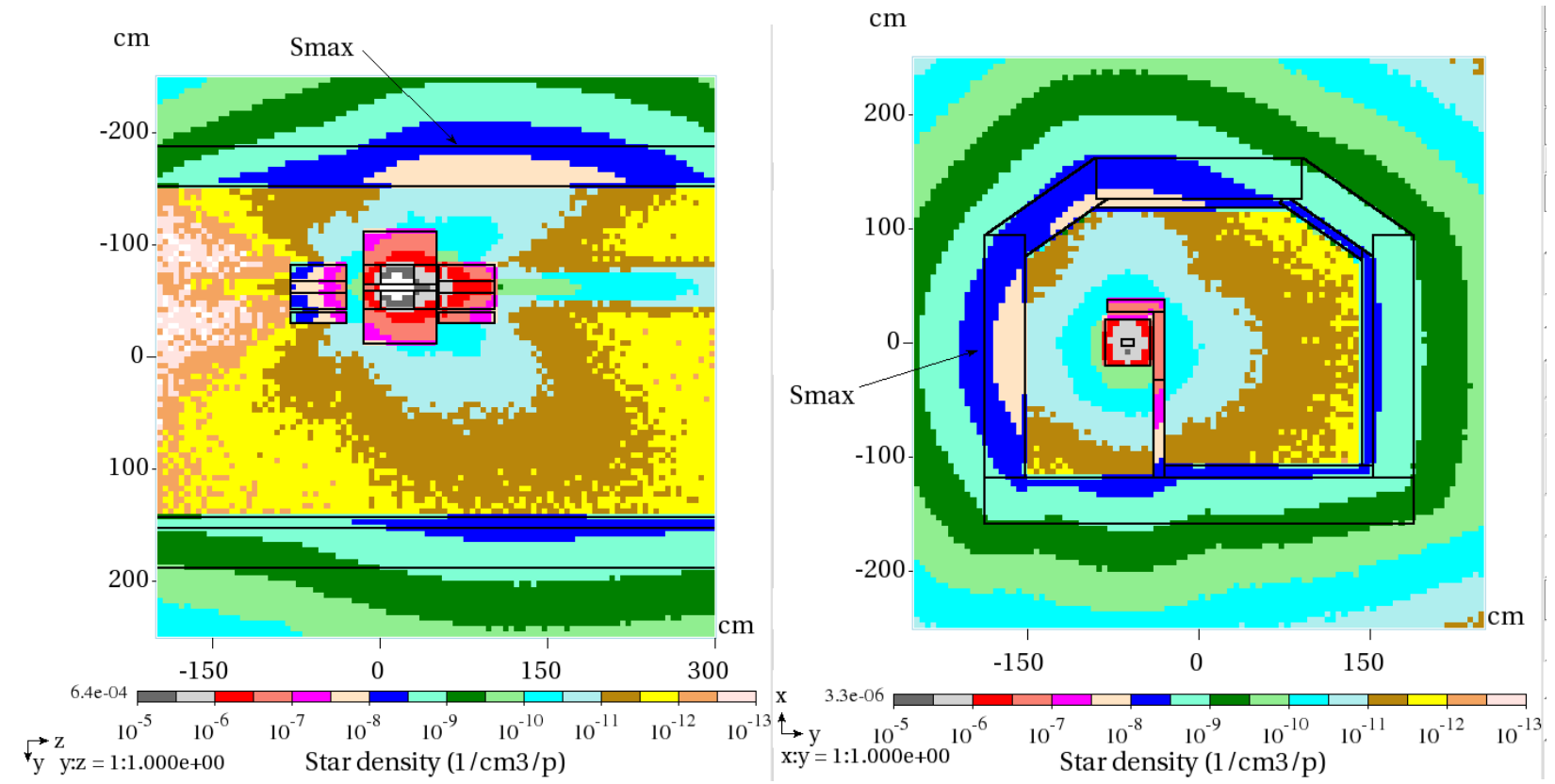

Figure 3.38: Calculated star density distribution around the absorber: plan view (left) and cross section (right).

The calculated distribution of the residual dose is shown in Figure 3.39. One can see that the maximum contact residual dose is below $1 \mathrm{mSv} / \mathrm{hr}$ on surfaces that can be easily or accidentally reached by personnel during routine maintenance procedures. Without the 4 " thick marble layers above and on the isle side of the upstream and downstream magnets, the contact residual dose could be well above $1 \mathrm{mSv} / \mathrm{hr}$. The maximum residual dose on the tunnel wall on the left side of the absorber is about $15 \mathrm{mSv} / \mathrm{hr}$, while on the right of the absorber the dose on the tunnel wall and floor does not exceed $0.3 \mathrm{mSv} / \mathrm{hr}$. The latter allows us to further optimize the shielding, if necessary, and reduce the amount of marble on the tunnel walls and floor as well as around the absorber and magnets upstream and downstream.

The calculated peak absorber dose in the magnet downstream of the absorber is about $4 \mathrm{MGy} / \mathrm{yr}$ (see Figure 3.40) while the expected lifetime of magnet components such as kapton, insulation (G10) and epoxy is 20-30 MGy. In other words, according to the current design the expected lifetime of the first magnet downstream is about 5-7 years. Shielding optimization in order to significantly increase the lifetime looks questionable because this magnet is practically in contact with the absorber. 


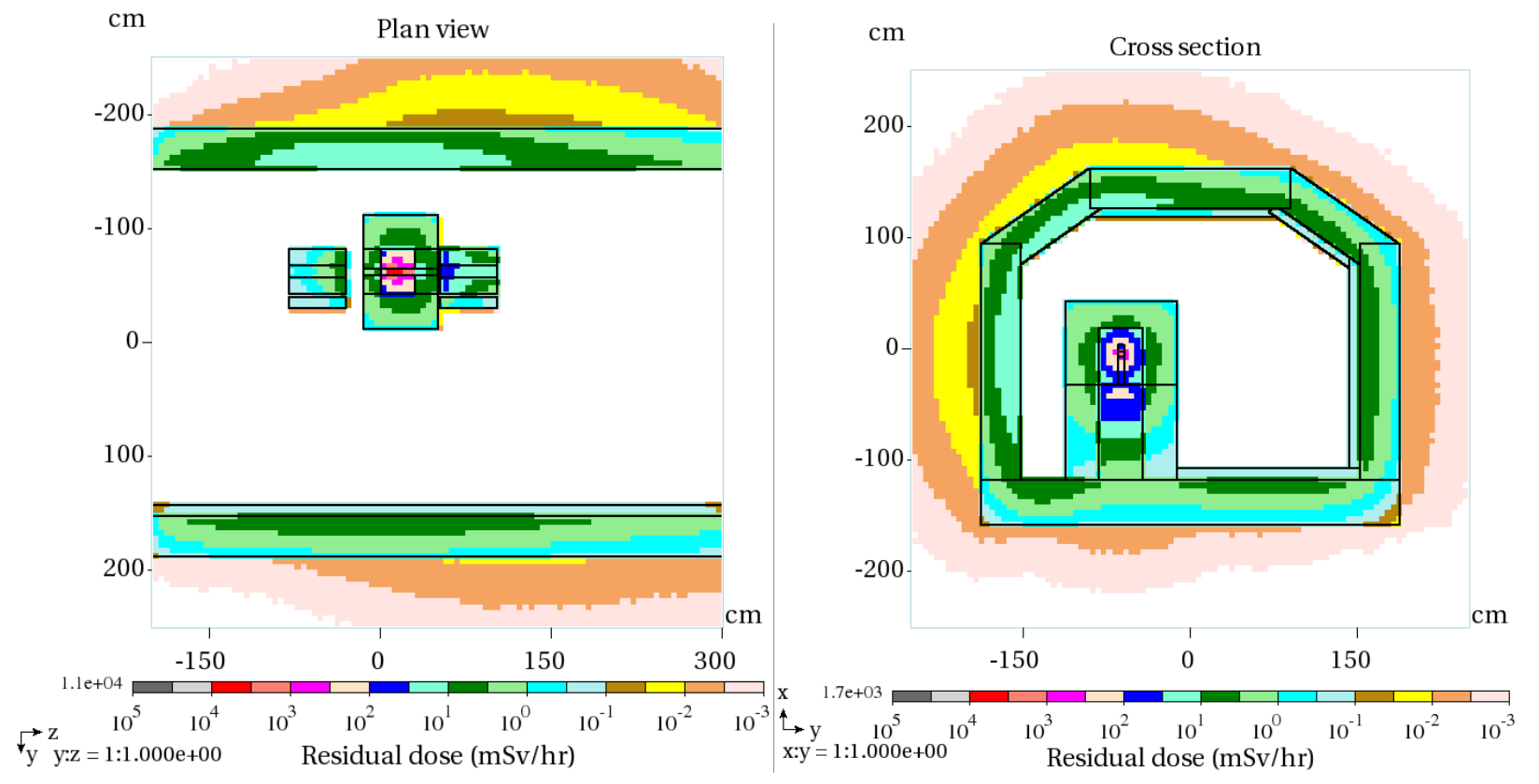

Figure 3.39: Calculated residual dose around the absorber for 30-day irradiation and 1-day cooling.
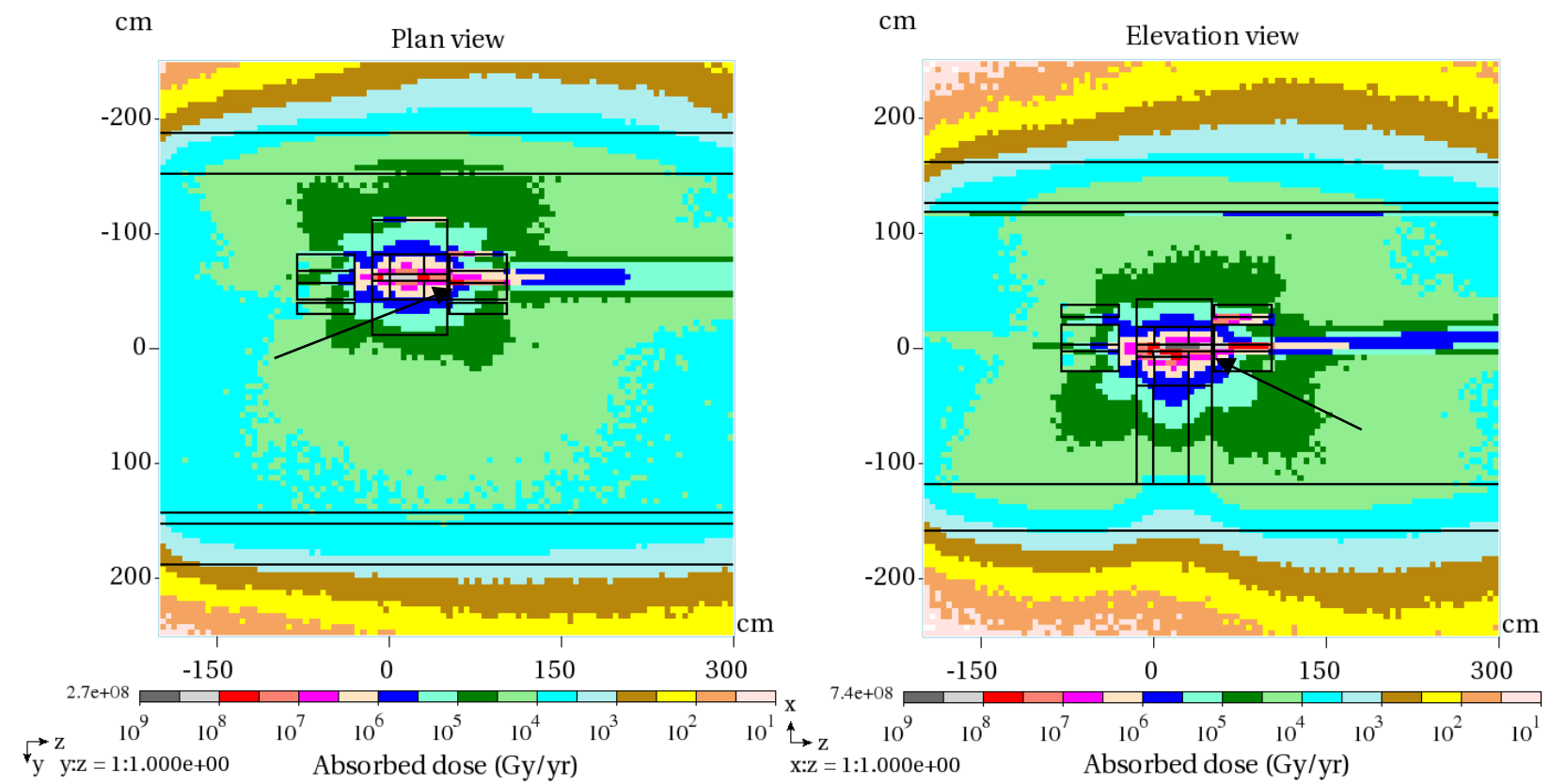

Figure 3.40: The calculated distribution of absorbed dose around the absorber. The peak absorbed dose in the magnet downstream is shown with arrows. 


\subsection{Main Injector and Recycler}

\subsubsection{Hardware for Main Injector Transition Crossing}

Designs for a $\gamma_{t}$-jump in the Main Injector have been studied for the last 15 years. Details can be found in the Proton Driver Design Report [82]; a brief summary is included here. The system consists of 8 sets of pulsed quadrupole triplets. Each triplet has two quads in the arc and one of twice the integrated strength in the straight section, with a phase advance of $\pi$ between each quadrupole. The perturbation to the original lattice is localized. In particular, the dispersion increase during the jump is small $\left(\Delta D_{\max } \approx \pm 1 \mathrm{~m}\right)$, which is the main advantage of a first-order jump system. Each triplet is optically independent from the others and provides roughly $1 / 8$ of the total required jump amplitude (i.e., $\Delta \gamma_{t} \approx \pm 0.25$ per triplet). The power supply uses a GTO (gate turn-off thyristor) as the fast switch and a resonant circuit with a $1 \mathrm{kHz}$ resonant frequency. The beam pipe is elliptical and made of Inconel 718. It has low electrical conductivity $\sigma$ and high mechanical strength so eddy current effects are relatively small. The eddy current effects scale as $\sigma d$, where $d$ is the pipe wall thickness. The $\sigma d$ value of Inconel 718 is about four times lower than that of stainless steel.

The 8 pulsed triplet locations are summarized in Table 3.12. Since the original study was done, there have been changes to the Main Injector and these locations need to be revisited. A set of magnet design parameters has been developed and modeled (see Table 3.13).

Table 3.12: Candidate $\gamma_{\mathrm{t}}$ quad triplet locations

\begin{tabular}{l|l}
\hline \hline Pulsed Triplet & Quad Locations \\
\hline 1 & $104,108,112$ \\
\hline 2 & $226,230,302$ \\
\hline 3 & $322,326,330$ \\
\hline 4 & $334,338,400$ \\
\hline 5 & $404,408,412$ \\
\hline 6 & $526,530,602$ \\
\hline 7 & $622,626,630$ \\
\hline 8 & $634,638,100$ \\
\hline \hline
\end{tabular}

Table 3.13: Pulsed quadrupole magnet parameters

\begin{tabular}{l|l}
\hline \hline & Requirement \\
\hline Integrated Gradient & $0.85 \mathrm{~T}$ \\
\hline Vacuum pipe cross section (elliptical) & $2.4 \times 1.125 \mathrm{in}$ \\
\hline Field Quality, 1 inch radius & $2 \%$ \\
\hline Maximum length & $17 \mathrm{in}$ \\
\hline Maximum Current & $200 \mathrm{~A}$ \\
\hline Maximum Voltage & As low as possible \\
\hline \hline
\end{tabular}




\subsubsection{RF System Modifications}

The Recycler and Main Injector need new $1^{\text {st }}$ and $2^{\text {nd }}$ harmonic RF cavities. The same cavities will be used in both machines. A cavity design has been developed, with perpendicular biased tuners and $\mathrm{R} / \mathrm{Q} \approx 30 \Omega$. A mechanical drawing of the cavity is shown in Figure 3.41 . The cavity parameters are shown in Table 3.14. Higher Order Mode (HOM) Coaxial dampers for the $53 \mathrm{MHz}$ cavities have been designed. The effect of the HOM dampers on the first 2 monopole cavity modes is shown in Figure 3.42. A mock-up of the first harmonic cavity has been constructed and a set of low level RF measurements were taken. A preliminary design of the second harmonic cavity that is a scaled down version of the first harmonic has been completed.

Cavity Material: Copper

Ferrite Material: TCl Ceramics AL-0400

Quantity 30

$32 \mathrm{~cm}$ OD x $12 \mathrm{~cm}$ ID $\times 1 \mathrm{~cm}$ thickness

$0.5 \mathrm{~cm}$ garnet separation

300 gauss to 2250 gauss to change $\mu_{\mathrm{r}}$ from 2.5 to 1.2

Permittivity $\left(\varepsilon_{r}\right)$ of 13.5

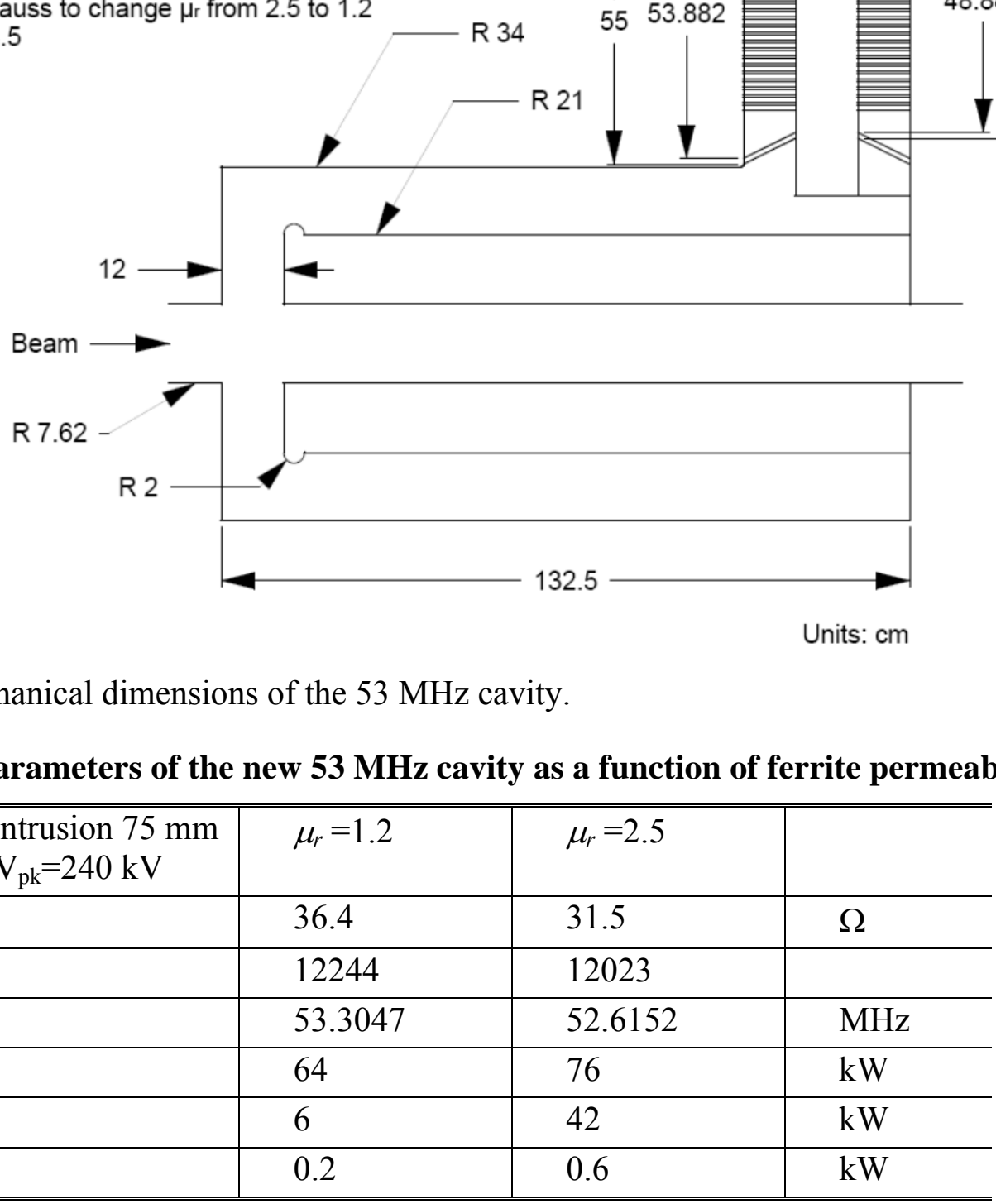

Figure 3.41: Mechanical dimensions of the $53 \mathrm{MHz}$ cavity.

Table 3.14: Parameters of the new $53 \mathrm{MHz}$ cavity as a function of ferrite permeability

\begin{tabular}{l|l|l|l}
\hline $\begin{array}{c}\text { Tuner Intrusion } 75 \mathrm{~mm} \\
@ \mathrm{~V}_{\mathrm{pk}}=240 \mathrm{kV}\end{array}$ & $\mu_{r}=1.2$ & $\mu_{r}=2.5$ & \\
\hline $\mathrm{R} / \mathrm{Q}$ & 36.4 & 31.5 & $\Omega$ \\
\hline $\mathrm{Q}_{0}$ & 12244 & 12023 & \\
\hline$f$ & 53.3047 & 52.6152 & $\mathrm{MHz}$ \\
\hline$P_{\text {wall }}$ & 64 & 76 & $\mathrm{~kW}$ \\
\hline$P_{\text {ferrite }}$ & 6 & 42 & $\mathrm{~kW}$ \\
\hline$P_{\text {ceramic }}$ & 0.2 & 0.6 & $\mathrm{~kW}$ \\
\hline \hline
\end{tabular}

The power source needs to provide greater than $550 \mathrm{kVA}$ of total power and $4.65 \mathrm{~A}$ of current. To simplify operation and maintenance, the source should have enough bandwidth to power both the $1^{\text {st }}$ and $2^{\text {nd }}$ harmonic cavities. The EIMAC 8973 power tetrode amplifier has a maximum 
operating frequency of $110 \mathrm{MHz}$, output power capabilities greater than $1 \mathrm{MW}$, and plate dissipation of $1 \mathrm{MW}$. An 8973 tube has been purchased and a power test stand is being developed.
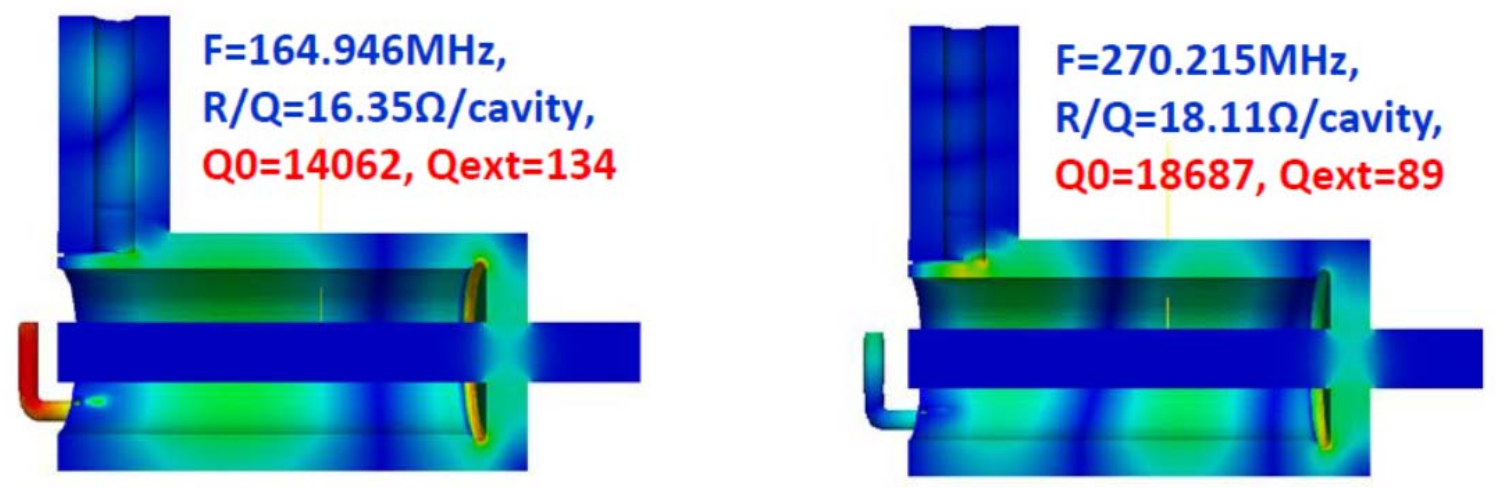

Figure 3.42: Effect of the $53 \mathrm{MHz}$ cavity HOM dampers on the first two monopole modes. 


\subsection{Cryogenics}

\subsubsection{Cryogenic System Configuration}

The Linac cryogenic system (see Figure 3.43) consists of three major subsystems: the cryogenic plant, the cryogenic distribution system, and the associated auxiliary systems. The system is expected to operate for 20 years, with an estimated continuous operation of two to five years without a scheduled shutdown. The expected availability of the cryogenic system is $98.5 \%$. The cryogenic system as a whole is required to perform the following functions:

- Provide sufficient cooling at appropriate temperature levels to enable operation of the SRF cavities and other cryogenic components within their respective operational conditions.

- Ensure that the system shall support controlled cool-down and warm-up of cryomodules.

- Ensure that the system and its components comply with the Fermilab ES\&H manual.

- Provide for proper protection of process fluids from contamination.

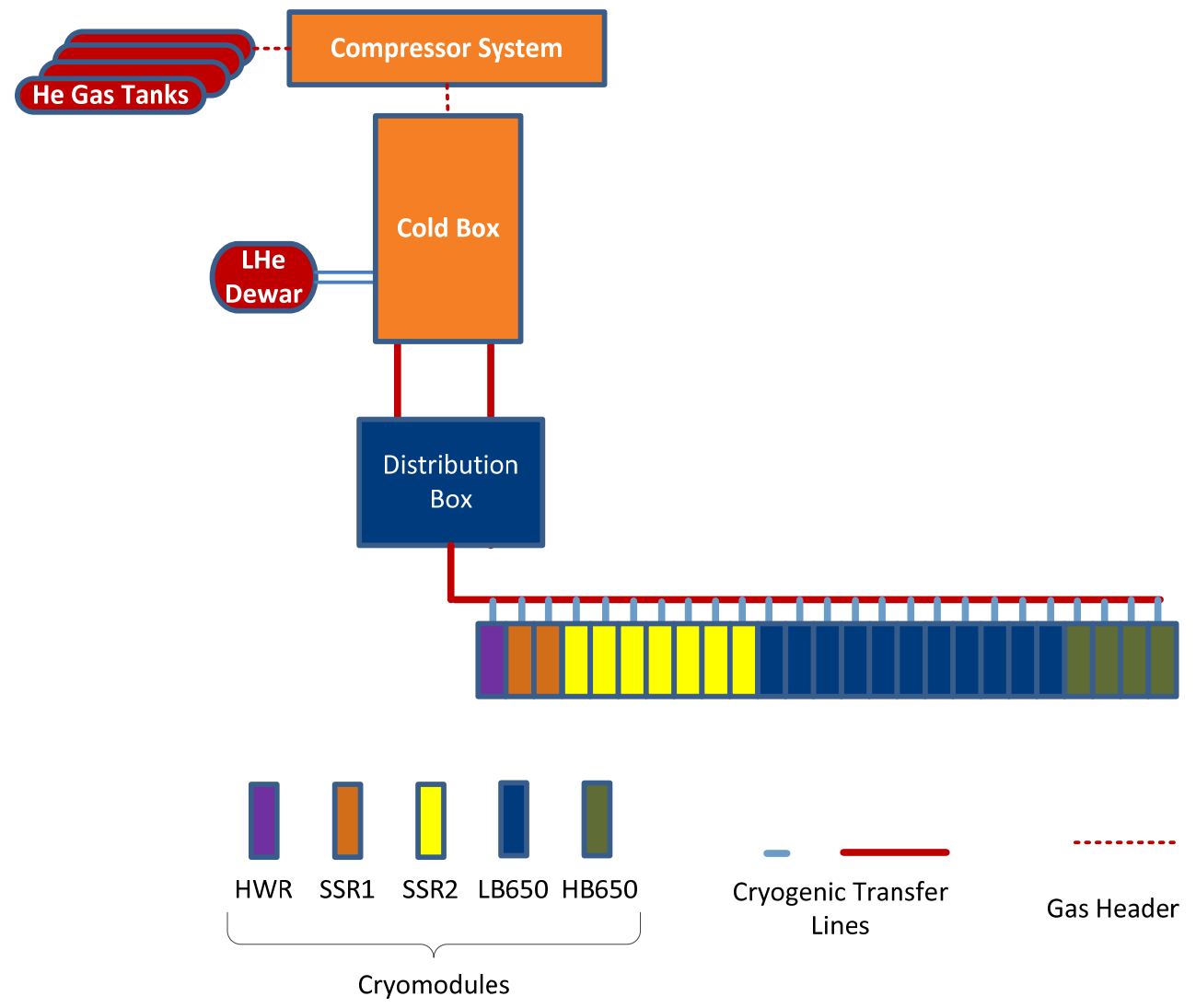

Figure 3.43: Layout of PIP-II cryogenic system.

The cryogenic system will be designed to operate as efficiently as is practical over a wide range of operating requirements [83]. Efficiency will be important for the operating modes that are expected to last for extended periods of time, such as normal Linac operation at $2 \mathrm{~K}, 2 \mathrm{~K}$ standby (RF off) and $4.5 \mathrm{~K}$ standby. Operating procedures for the cryogenic system include: 
1. Controlled linac cool down and warm up.

The cryogenic system must be able to reliably cool down and warm up the cryomodule string within the cool down rate and temperature difference constraints imposed by the cryomodule design.

2. Linac liquid helium fill

This represents the $4.5 \mathrm{~K}$ liquefaction capacity of the cryogenic system and determines the time required to fill the cryomodule string with $4.5 \mathrm{~K}$ liquid helium.

3. $4.5 \mathrm{~K}$ standby

During extended shutdown periods, it is desirable to keep the cryomodule string cold while minimizing the operating cost as well as the risks by operating all circuits at positive pressure.

4. $2 \mathrm{~K}$ standby

During shorter shutdown periods, it is desirable to keep the cryomodule string at $2 \mathrm{~K}$. With the RF off, the heat load to the cryogenic system at $2 \mathrm{~K}$ will be about $50 \%$ of the nominal load in the pulsed regime of linac operation.

5. $2 \mathrm{~K}$ design operation

This represents the normal pulsed operation of the Linac at the estimated heat loads.

The cryogenic system will utilize a mixed compression cycle, as opposed to all cold compression cycle. The mixed cycle has been successfully used in many superfluid helium cryogenic plants including LHC (CERN) and CMTF (Fermilab).

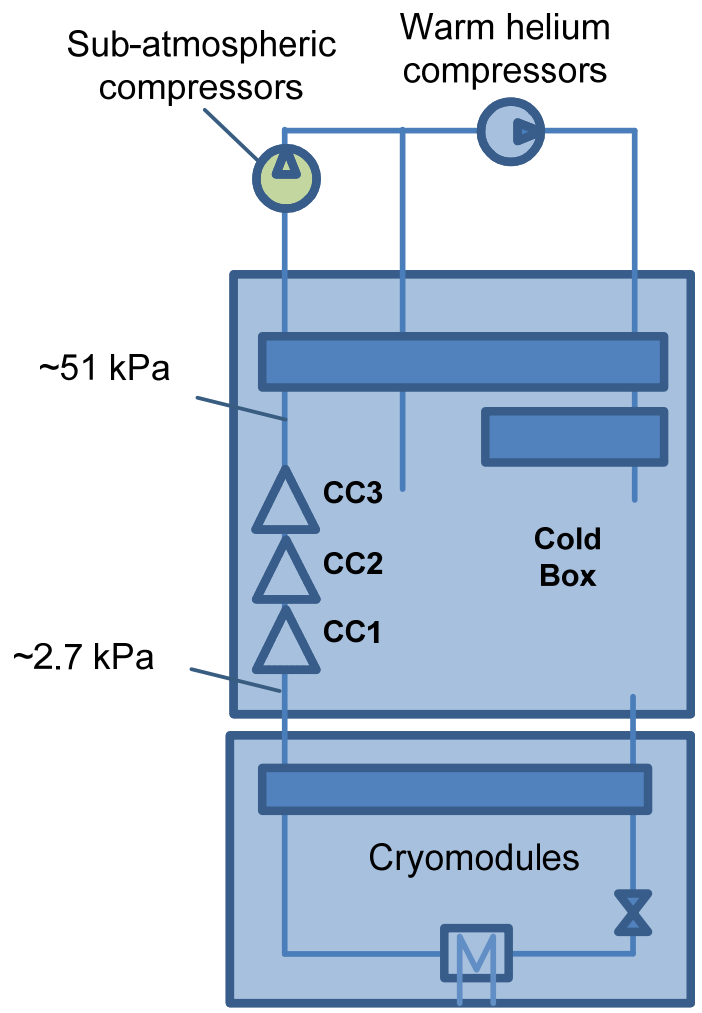

Figure 3.44: Simplified Schematic of a Mixed Compression Cycle.

The cycle was also recently chosen by XFEL (DESY) and European Spallation Source (ESS) 
after independent review of an industrial studies conducted by the helium cryogenic plant manufacturers. Simplified cycle diagram of the cycle is presented in Figure 3.44. The cycle utilizes a combination of three stages cold compressors and a sub atmospheric warm compressor operating in series. The major advantage of this cycle is the wide range of efficient capacity modulation.

The PIP-II cryogenic plant design will incorporate the components to accommodate all required operating modes of the/PIP-II Linac as well as devices necessary for its function verification. The cooling capacity of the cryogenic plant is presented in Table 2.9. The plant will include the following major components and sub systems:

- Warm compressor system (WCS),

- Sub-atmospheric compressor system (SCS),

- Compressor oil removal system,

- Motor control centers and variable frequency drives,

- Gas management panel (GMP),

- A single cold box (heat exchangers, turbines, valves, cold compressors etc.),

- A purge panel,

- Plant control system,

- Plant instrumentation.

The cold box will be procured from industry. It will consist of a vacuum vessel with shell and top plate with clean inner surfaces, covered by multi-layer insulation. The cold box also includes instrument panel with transmitters, switches, process and utility instrumentation, instrument air and cooling water connection and distribution, purge connection, electric cabinet for terminals, transmitters and other hardware, integrated full flow dual bed $80 \mathrm{~K}$ adsorber, integrated full flow single bed $20 \mathrm{~K}$ adsorber, a set of aluminum plate-fin heat exchangers, cryogenic valves with manual or pneumatic actuators, temperature sensors and pressure sensing taps, transfer line connections, cryogenic turbo expanders and three cryogenic cold compressors, and all required safety relief devices. The cold box exterior is painted to protect it from corrosion.

It is assumed that four Mycom 2016-C helium compressors located at the A0 compressor building can be used to support the cold box operation. The compressors are currently assigned to the experimental program at the Muon Campus. It is also assumed that these compressors will become available at the time of PIP-II operations. As an alternative, a new compressor building would be needed and other Tevatron compressors could be moved to it. The latter solution would require additional power and cooling water capability in the vicinity of the Linac.

Each of four compressor skids has a direct-drive two-stage, oil flooded rotary twin helium screw compressor which utilizes asymmetrical rotor profiles and is directly driven by a $300 \mathrm{~kW}, 3600$ rpm motor. Each skid contains helium and oil cooling equipment, bulk oil removal system, oil and gas filters, local instrumentation and controls. The SCS will be procured from industry. The SCS machines will have variable frequency drives.

Cryogenic plant controls system will be equipped with all instrumentation necessary for safe and reliable operation. The instrumentation will allow for flow and pressure measurements, temperature, speed controls and impurity monitoring. The control system will consists of a main process control programmable logic controller (PLC), remote input/output modules at equipment positions and Human-Machine-Interface displays. It will include all software that is required for safe and reliable operation of the system.

Most of the auxiliary equipment, including warm helium storage tanks, liquid helium dewars, 
purification equipment, etc. will be reused from the Tevatron. Chillers for turbines and cold compressors, insulation vacuum pumping system (roughing and diffusion pumps), and associated instrumentation will be procured from industry.

The cryogenic distribution system consists of the equipment needed to feed and return the cryogens via vacuum insulated pipelines to the Linac components needing these services throughout the entire Linac. This equipment includes distribution box, cryogenic transfer lines, bayonet and turnaround boxes.

A new multi-circuit transfer line will run from the new refrigerator building, into the tunnel and along the length of the SC Linac. The design of this transfer line will be consistent with $\mathrm{CW}$ operation. The transfer line requires 25 inline bayonet cans, one for each cryomodule, and a turnaround box at the end. In parallel, there will be a warm helium vent header.

Connection between the distribution boxes and the cold box is accomplished via removable vacuum insulated cryogenic transfer tubes ("U-tubes"), while the connection to the tunnel transfer line is welded. This architecture provides flexibility for positive isolation of tunnel components and strings of cryomodules from the cryogenic plant during installation, commissioning, operation and maintenance, including repairs. Each cryomodule is connected to the transfer line via u-tubes, which provides for maximum segmentation of the Linac.

\subsubsection{Infrastructure and Utilities Requirements}

The PIPI-II cryogenic system requires the following infrastructure/utilities support:

- Building floor space: 15'h x 30'w x 50'1,

- Electrical Power: 1.2 MW, distribution panels, 480V/3ph,

- Cooling water: 800 gpm,

- Ventilation: 4 x 4,500 CFM fans, outside air intake louvers, space heaters,

- General lighting.

The cryogenic system has to be designed to minimize excitation of ground motion which can excite microphonics in the SC cavities. 


\subsection{Instrumentation}

Various beam instrumentation and diagnostics systems are necessary to characterize the beam parameters and the performance in all PIP-II sub-accelerators. For startup and initial beam commissioning we need to provide, at a minimum, beam instruments in order to observe:

- Beam intensity,

- Beam position / orbit,

- Transverse beam profiles,

- Beam phase / timing.

The high beam intensity / power and the presence of superconducting technologies also require a reliable, fail safe machine protection system (MPS) to prevent quenches in cryogenic elements or damage due to an uncontrolled loss of the high power beam. This system will be based on beam loss monitors (BLM) and other beam intensity monitors (e.g. toroids).

Beside these core beam instrumentation systems, more specialized beam diagnostics need to be provided, e.g. to characterize the longitudinal bunch profile and tails, transverse beam halo, bunch-by-bunch chopping efficiency and more advanced beam emittance measures. Many types of beam monitors (e.g. BPMs, toroids, etc.) can be standardized. However some areas in PIP-II, such as the frontend ( $\mathrm{H}^{-}$source, RFQ and MEBT) will demand dedicated beam diagnostics (Allison scanner, fast Faraday cup, e-beam scanner, vibrating-wire, etc.).

For the linac the beam monitoring within the cryogenic environment is limited to beam orbit monitoring with BPMs. Most other beam diagnostics will be located at warm sections between cryomodules. In addition, if the space is available, we will also investigate the possibility of incorporating laser profile monitors between cryo-modules.

In order to develop this beam instrumentation, a complete set of "beam measurement requirements" has to be established. Each sub-accelerator (linac, transport lines, Booster, MI, Recycler) needs to address the operating modes with the nominal, as well as non-standard beam parameters, and all requirements for the different beam measurements (resolution, precision, dynamic range, etc.). We foresee the following general detectors and systems for beam instrumentation and diagnostics.

\section{Beam Position Monitors}

The beam orbit monitoring is the most fundamental measurement and the most powerful diagnostics tool in an accelerator. PIP-II requires a large number $(\sim 100)$ of new warm and cold beam position monitors (BPM), thus making it a complex and expensive measurement system. 37 BPM pickups will be located inside HWR, SSR1 and SSR2 cryo-modules. Their design has to be done to meet UHV, cryogenic and clean room requirements simultaneously. Recently the prototype of the HWR and SSR1 BPM pickups was successfully tested in the Argonne National Laboratory. All linac BPMs are based on four electrode pickups and have to be capable to measure all 3 coordinates, horizontal, vertical and RF phase, as well as a measure of the beam intensity. BPMs in the linac-to-Booster line are single coordinate pickups: horizontal or vertical depending on their location (horizontal near F quad, vertical near D quad). 
The spectrum of BPM signals is concentrated in high frequency (up to a few $\mathrm{GHz}$ ). It requires high quality RF cables to transmit their low-power high frequency signals to the read-out hardware outside the accelerator tunnel. This requirement may impact the arrangement or layout of some conventional facilities.

The major requirements on BPM accuracy come from optics measurements based on the differential orbits. It requires the relative position measurements better than $30 \mu \mathrm{m}$, the relative RF phase measurements better than $0.1 \mathrm{deg}$. and relative beam intensity under $1 \%$,

\section{Beam Profile Monitors}

Profile monitors are required at a few locations in the MEBT, the SC Linac and the Linac-toBooster transfer line for measuring the beam emittances and phase space to match the beam to the Booster injection. Options for transverse profile monitors in the $\mathrm{H}^{-}$sections of PIP-II are the standard multi-wire monitor and the newer laser profile monitor. Laser-based profile monitors are intended as the primary technology choice with standard multi-wires as a fallback technology. Laser profile monitors also allow measurements of the longitudinal beam profile. In addition, profile measurements in the rings will be made using ionization profile monitors and electron wire profile monitors.

\section{Beam Loss Monitors}

Typical fast ionization chambers with a large dynamic range will be utilized for most loss measurements. However, there may be instances where measurements of thermal neutrons or machine activation are desired. The loss monitors will be incorporated in the machine protection system (MPS). Beam loss monitoring with ionization chambers in the low-energy section of the linac are not possible. We intend to measure beam loss, in the linac frontend, through precision beam current measurements as well as by measuring beam current on electrically-isolated scrapper plates.

\section{Beam Current Monitors}

To determine the beam current and the beam loss (in absolute units) a combination of DCCTs and beam toroids will be used. They have to allow the high precision beam current measurements for both pulsed and $\mathrm{CW}$ operation of the linac. In addition, we estimate that, in the linac, we can obtain beam current measurements to better than $1 \%$ through the BPM system.

\section{Special Monitors}

Several types of special beam monitors and diagnostic tools are required to verify the beam quality and minimize beam losses. These include monitoring of the transverse beam halo $(e . g$. vibrating wires, laser wires and scrappers), the measurement of bunch-by-bunch chopping efficiency ( $e . g$. high bandwidth wall current monitors and deflecting cavities) and the detection of longitudinal tails (e. g. laser wires and high bandwidth wall current monitors). A list of special beam monitors and diagnostic tools is not complete but design of generic instrumentation ports in diagnostic sections will allow future instruments to be installed. Development and test of such monitors will be carried out in PXIE. 


\section{Data Acquisition and Timing}

Most beam monitoring systems will use frontend digital signal conditioning and processing methods to extract the wanted beam parameter(s). The generated output data needs to be "time stamped" with respect to the beam event, so beam and other recorded data can be crosscorrelated throughout the entire PIP-II complex. This cross correlation will simplify diagnostics and trouble-shooting on the day-to-day operation.

\section{Instrument Physical Space Issues}

Sufficient physical space is allocated in the linac optics design to accommodate the required beam detection elements. At some critical, real-estate limited locations, e.g. LEBT, MEBT, injection / extraction, and SRF areas, a compromise must be worked out, which enables an acceptable way to sense the beam without compromising its quality in the diagnostic sections. 


\subsection{Controls}

The control system is responsible for control and monitoring of accelerator equipment, machine configuration, timing and synchronization, diagnostics, data archiving, and alarms. PIPII will use an evolution of the Fermilab control system ACNET [84], this is the system that is used in the main accelerator complex and also at the NML/ASTA and PXIE test facilities [85]. ACNET (Figure 3.45) is fundamentally a three tiered system with frontend, central service, and user console layers. Front-end computers directly communicate with hardware over a wide variety of field buses. User console computers provide the human interface to the system. Central service computers provide general services such as a database, alarms, application management, and frontend support. Communication between the various computers is carried out using a connectionless protocol over UDP. Subsystems developed by collaborators based on the EPICS control system can be integrated into the main system.

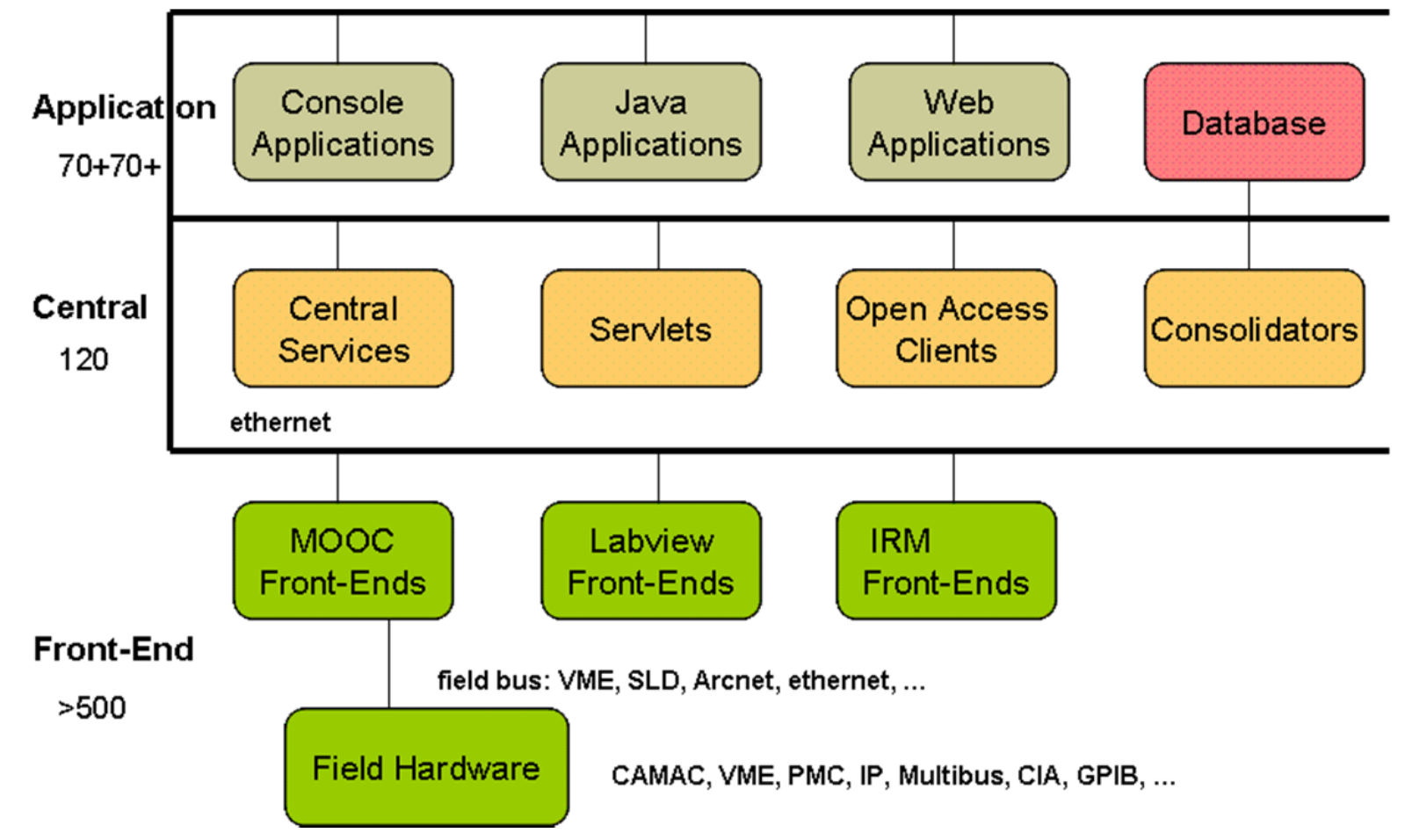

Figure 3.45: PIP-II Controls System architecture.

The scale of the control system is expected to be similar to that of the complex when the Tevatron was operating. The system should support up to $1 \mathrm{M}$ device properties. Time stamping must be provided so that all data from the SC Linac can be properly correlated with that from the existing complex. The control system should contribute less than $1 \%$ to operational unavailability. The high beam power implies the need for a sophisticated machine protection system to avoid damage to the accelerator due to errant beam pulses.

The Fermilab Accelerator Control System makes use of a 2 tiered timing system for the accelerators. The top level is a $10 \mathrm{MHz}$ based clock system (TCLK) that provides basic system co-ordination and data acquisition timing. The second level clock system is specific to each 
accelerator and is synchronous to beam in that machine. The beam sync clocks have base frequencies that are derived from the machine's RF to provide bunch level timing for devices such as intensity monitors, BPMs and kickers. Relevant clock events are reflected from one level of clock system to the other as needed to support operations. A new upper level timing system will be developed that is a major enhancement over the TCLK and MDAT links in the main complex. A prototype has been developed based on a $1 \mathrm{Gbps}$ data link that adds a data payload and cycle stamp to each clock event transmission. The latter will allow reliable correlation of data across different frontends. The prototype design will be updated based on requirements for the SC Linac to serve as its beam synchronous clock.

It is highly desirable to have a single control system operating the entire complex rather than separate systems for the new linac and older parts of the system. There should only be a single copy of core services such as alarms and data archiving. Software applications should be able to access any device in the system. This model simplifies development and operation, and reduces long term maintenance costs of the complex. The current control system covering the Booster, Main Injector and Recycler represents a large investment in both software and hardware and it is not practical to completely replace it by the start of PIP-II operations. The strategy will be to gradually update parts of the system before and during PIP-II construction, and in support of the NML and PXIE test facilities. This upgrade will include modernizing the application software environment as well as replacing obsolete hardware. Upgraded timing and machine protection systems will be developed for the PIP-II linac that will accommodate the legacy hardware in the existing parts of the complex. These systems as well as the linac control software will be prototyped at NML and PXIE.

It is recognized that some equipment will be developed outside of Fermilab by institutions with expertise in the EPICS control system. Also it may be appropriate in some cases to use commercial hardware that comes with embedded EPICS software. It is planned to support integration of EPICS frontends and some core applications in the Fermilab control system. This has been demonstrated in several different ways at different levels of the control system at the HINS and NML test facilities. The control system will specify standard interfaces between its internal components as well as with technical equipment. This will make integration, testing, and software development easier and more reliable and reduce the long term maintenance load. Also standard interfaces allow parts of the system to be more easily upgraded if required for either improved performance or to replace obsolete technologies. Only portions of the system need be changed while the core architecture of the control system remains the same.

\section{Operation at $20 \mathrm{~Hz}$}

Increasing the Booster repetition rate from 15 to $20 \mathrm{~Hz}$ will be a significant change in the control system. The current timing system is based on a $15 \mathrm{~Hz}$ signal derived from the $60 \mathrm{~Hz}$ line voltage along with a $15 \mathrm{~Hz}$ signal generated by the booster GMPS. These are transmitted out to the rest of the complex as TCLK events generated via the Timeline Generator (TLG). These events will have to be changed to $20 \mathrm{~Hz}$ events. The shorter time between events and beam pulses will have to be accounted for by software changes to the TLG which generates the main timing signals for the various accelerators. A variety of systems perform software tasks on each $15 \mathrm{~Hz}$ pulse and each will have to be examined to ensure there is sufficient time to complete their task when the timing moves to $20 \mathrm{~Hz}$. Though this is a major change that impacts many parts of 
the control system, it is currently believed that both PIP-II and the remaining parts of the existing complex should be able to adapt. 


\subsection{Radiation Safety and Radiation Shielding Design}

Design requirements and radiation limits for accelerators and beam transport lines are provided by the Fermilab Radiological Controls Manual (FRCM). The manual requires wellengineered designs that maintain occupational and environmental radiation exposures as low as reasonably achievable (ALARA) and that are compliant with applicable regulations and DOE Orders. The first choice for accelerator shielding designs is to have passive shielding elements designed to enable areas external to shielding to be classified as minimal occupancy. Minimal occupancy is defined to mean any area which is not normally occupied by people more than 1 hour in 8 consecutive hours. Dose rates for potential exposure to radiological workers in areas without continuous occupancy are to be ALARA and such that individuals do not receive more than $20 \%$ of the applicable limits. The design goal for dose rates in areas of continuous occupancy is to be less than an average of $0.05 \mathrm{mrem} / \mathrm{hr}$ and as far below this and ALARA. Reliance on active systems such as radiation safety interlocks and/or beam line instrumentation to achieve radiation safety goals should be chosen only if passive elements cannot, in view of planned accelerator operations, reasonably achieve the level of protection required by the FRCM. Discharges of radioactive liquid to the environment should be kept ALARA. Materials and components should be selected to minimize the radiological concerns, both occupational and environmental. Where removable contamination might be associated with accelerator operations, provisions should be made in facility designs for the containment of such material. Internal radiation exposure is to be minimized in accordance with ALARA principles by the inclusion of engineered controls such as ventilation, containment, filtration systems, where practicable and with appropriate administrative procedures. Efficiency of maintenance, decontamination, operations, and decommissioning should be maximized.

The FRCM specifies the manner in which radiological posting requirements are to be determined. The maximum dose is that which can be delivered under the worst credible accident in that area, taking into consideration circumstances and controls, which serve to limit the intensity of the maximum beam loss and/or its duration. Some examples of accident scenarios are (1) beam intensity significantly greater than the nominal beam intensity; (2) unanticipated beam losses; and (3) single pulse full machine loss on an element. The maximum dose is to be determined through the Safety Analysis, which shall document calculations and measurements of possible radiation exposures, radiation shielding, beam optics and other relevant information. The Safety Analysis must be reviewed and approved by the SRSO prior to construction and/or operation of the beam.

The FRCM specifies requirements for entry controls. Accelerator/beam line areas are to be posted and controlled for the normal operating conditions when the Safety Analysis documents that delivering the maximum dose to an individual is unlikely. Accelerator/beam line areas are to be posted and controlled for accident conditions when the Safety Analysis documents a scenario in which it is likely that the maximum dose may be delivered to an individual.

A Safety Analysis for PIP-II beam operation and the application of the FRCM design requirements to PIP-II are described in the remainder of this section. 


\subsubsection{Radiation Limits}

\section{Safety Analysis}

A PIP-II upgrade will change the operation of SC Linac from the pulsed to $\mathrm{CW}$ regime. That will enable linac operation with megawatt scale beam power. To determine the range of normal and accident beam loss conditions this Safety Analysis considers both the machine operation in the pulsed and $\mathrm{CW}$ regimes as described in Section 1.

Accelerator components such as cryomodules and beam pipes can be damaged or destroyed very quickly by beam power even at the levels envisioned for PIP-II. It becomes much more challenging for the PIP-II upgrade. The PIP-II SC Linac and associated beam lines require unprecedented control of beam orbit, beam optics, and beam losses in order to provide decades of safe operation for experimental programs. Consequently, the control of beam loss through a machine protection system is a primary design consideration for PIP-II.

The principal design features required for the control of beam loss in the SC Linac and Linac to Booster Beam Transport include precision alignment of all accelerator components, precise control of beam focusing, elimination of RF jitter, and precision control of beam orbit. Operation of PIP-II accelerators and beam lines without precision controls could easily result in beam losses exceeding $100 \mathrm{~W} / \mathrm{m}$. Machine protection systems are required to ensure that all beam control features are functional and operating as intended. The loss of any precision control feature will cause the machine protection system to inhibit beam acceleration at the ion source and LEBT. The machine protection systems will be capable of limiting or stopping machine operation within a few microseconds of sensing an abnormal condition.

PIP-II accelerators and beam lines accelerate and transport $\mathrm{H}^{-}$beams. The principal beam loss mechanisms are related to stripping electrons from $\mathrm{H}^{-}$ions; the causes of stripping include $\mathrm{H}^{-}$ ion collisions with residual gas, blackbody photon interactions, Lorentz force (magnetic stripping), and intrabeam stripping. The major contribution to beam loss comes from intrabeam stripping and this has been determined to be below $0.1 \mathrm{~W} / \mathrm{m}$ (see Section 2.1.4). Losses from the remaining mechanisms are significantly smaller.

The beam power delivered by the Booster Accelerator has risen significantly over the lifetime of this accelerator, while the radiation shielding available over the Booster beam enclosure has remained fixed. Improvements resulting from PIP and other machine upgrades have led to increasing beam power while controlling and reducing beam losses. An interlocked radiation detector array has been necessary protective measure at the Booster accelerator to compensate for the fixed shielding inventory. Some remaining PIP upgrades are yet to be implemented which will raise the Booster beam power to $80 \mathrm{~kW}$ while keeping the beam losses at a fixed or reduced levels. Since it is not practicable to increase Booster radiation shielding, it is necessary to continue with reliance upon an interlocked radiation detector system to limit the severity and duration of beam loss conditions. Reliance upon an interlocked radiation detector system makes it implicit that radiation levels are controlled at defined, normal loss conditions. That is, the normal loss condition is the de facto maximum beam loss condition. The maximum normal loss condition, is limited by the FRCM, while the nominal upper limit is set at some lower level by the laboratory's well established shielding review process.

The MI8 line and the Main Injector accelerator are heavily shielded with 24.5 feet of earth 
equivalent shielding. The addition of radiation shielding to the MI8 line and the Main Injector accelerator would be both costly and of limited utility. For example, at a beam energy of $8 \mathrm{GeV}$, a $22.5 \mathrm{~kW}$ beam loss is required to exceed the $1 \mathrm{mrem} / \mathrm{hr}$ on the surface of the shielding berm. Various machine protection features inhibit the continuous loss of beam power at this level. As is the case for the Booster accelerator, the shielding assessment process is employed to ensure the limits of the FRCM are observed for the MI8 line and the Main Injector. If found to be necessary, a comprehensive, interlocked radiation detection system (TLM) described below can be employed to ensure compliance with all requirements of the FRCM.

The shielding prescription required by this Safety Analysis for the SC Linac and the SC Linac to Booster Transfer Line is discussed below.

\section{Facility Design Beam Loss Level}

The conclusion of the above Safety Analysis is that the average beam loss for the SC Linac and the Linac to Booster Beam Transport Line under normal conditions will be of the order of $0.1 \mathrm{~W} / \mathrm{m}$. Machine protection systems will monitor the performance of beam focusing, beam orbit, RF stability, and machine alignment. Machine protection systems will reduce accelerator beam power or inhibit accelerator operation in the event the precision control of the accelerator is lost. The reaction time of the machine protection systems under consideration is on the order of a few microseconds. Therefore, only operation under normal conditions should be possible. For the purposes of the facility shielding design, it is assumed that the peak average beam loss will be $1 \mathrm{~W} / \mathrm{m}$, a factor of 10 higher than what is expected during nominal beam operating conditions.

\section{Facility radiological design goals}

The design goals for the PIP-II SC Linac and the SC Linac to Booster Beam Transport Line meet or exceed the minimum requirements of the FRCM stated above. The design goals are:

1. Permit unlimited occupancy for all service buildings, shielding berms, parking lots, control rooms, and associated areas. By design, radiation levels are to be kept below $0.05 \mathrm{mrem} / \mathrm{hr}$ in all accessible locations outside of the beam enclosures for normal operating conditions, based upon an assumed continuous beam loss of $1 \mathrm{~W} / \mathrm{m}$. The actual nominal beam loss condition described in the Safety Analysis is expected to be about $0.1 \mathrm{~W} / \mathrm{m}$.

2. Permit inspection and maintenance activities within tunnel enclosures while maintaining personnel radiation exposure due to residual activation of accelerator components and beam enclosures at levels as low as reasonably achievable. At 0.1 $\mathrm{W} / \mathrm{m}$, the residual dose rates should not exceed about $15 \mathrm{mrem} / \mathrm{hr}$.

3. Limit radiation exposure due to air activation both within the beam enclosure during inspection and maintenance activities and at the site boundary.

4. Limit ground water and surface water activation to levels well below regulatory standards.

5. Prevent the activation of beam component surfaces to avoid the generation of removable radioactivity.

6. Minimize the activation of accelerator components which can impact their useful 
service life.

\subsubsection{Radiological Design Requirements}

Nominal beam loss throughout the PIP-II accelerator and beam lines is expected to be about $0.1 \mathrm{~W} / \mathrm{m}$. Machine Protection Systems will limit or inhibit beam operations within microseconds of sensing a machine fault. The design requirements for radiation shielding discussed below are based upon an assumed continuous beam loss of $1 \mathrm{~W} / \mathrm{m}$. The consequences of the activation of accelerator components, enclosure structures, air, water, and removable contamination are discussed in terms of the expected nominal beam loss of $0.1 \mathrm{~W} / \mathrm{m}$ as defined in the Safety Analysis.

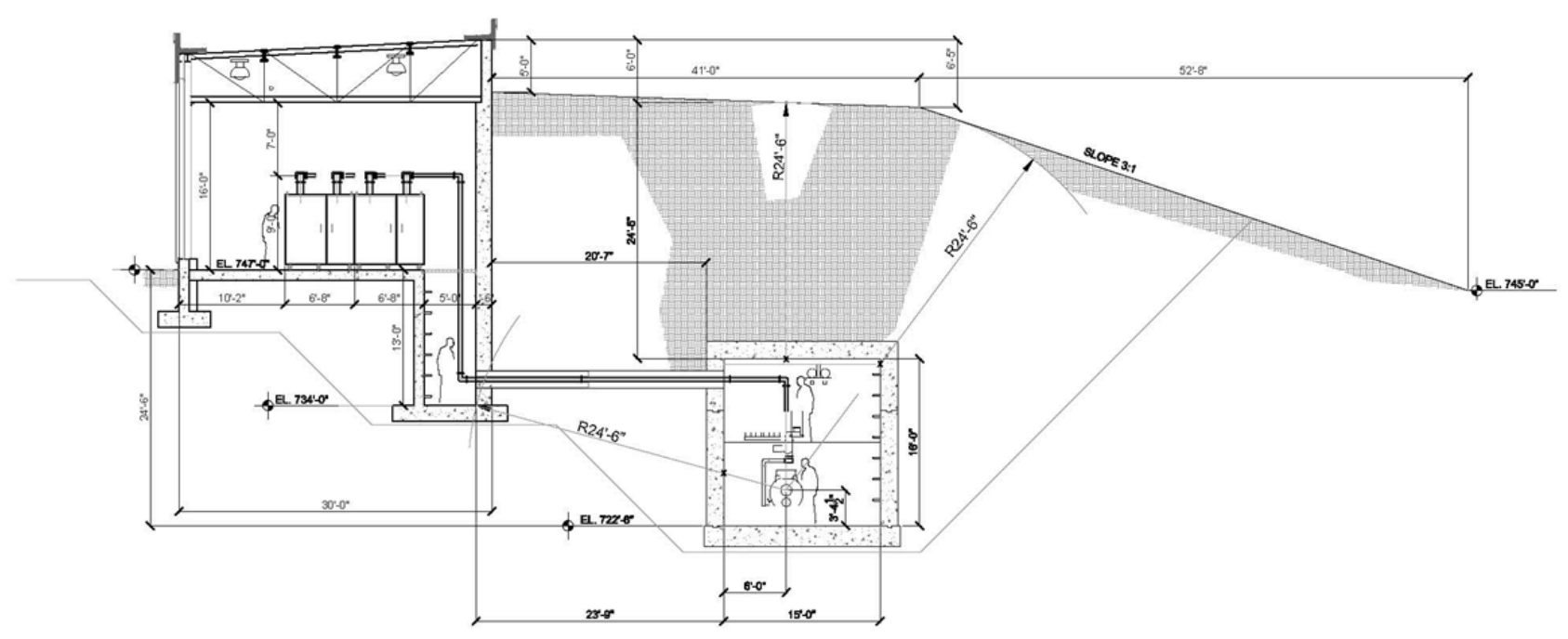

Figure 3.46: An early concept for the cross section of PIP-II linear accelerator enclosure

\section{Radiation Shielding}

An early conceptual design of a PIP-II accelerator enclosure is shown in Figure 3.46. An enclosure height of 16 feet is indicated along with passive shielding of 24.5 feet. An option to transport $0.8 \mathrm{GeV}$ beam to the Booster is shown in Figure 3.47. The transport line will be crossing the beam line delivering the $120 \mathrm{GeV}$ MI beam to the experimental areas (former Tevatron/Main Ring tunnel). The tunnel height is 8 feet and the shield is approximately 20 feet. At this time, details of the PIP-II layout and facility design have not been finalized. It is necessary that the accelerator design precede the shield design, but some shielding design concepts for PIP-II are considered here.

An established parameterization [86] is used to determine the radiation dose equivalent rate as a function of energy $(\mathrm{GeV})$, distance (feet), and angle with respect to incident beam direction (degrees) from a low energy proton beam $(<1 \mathrm{GeV})$ incident upon a target: 


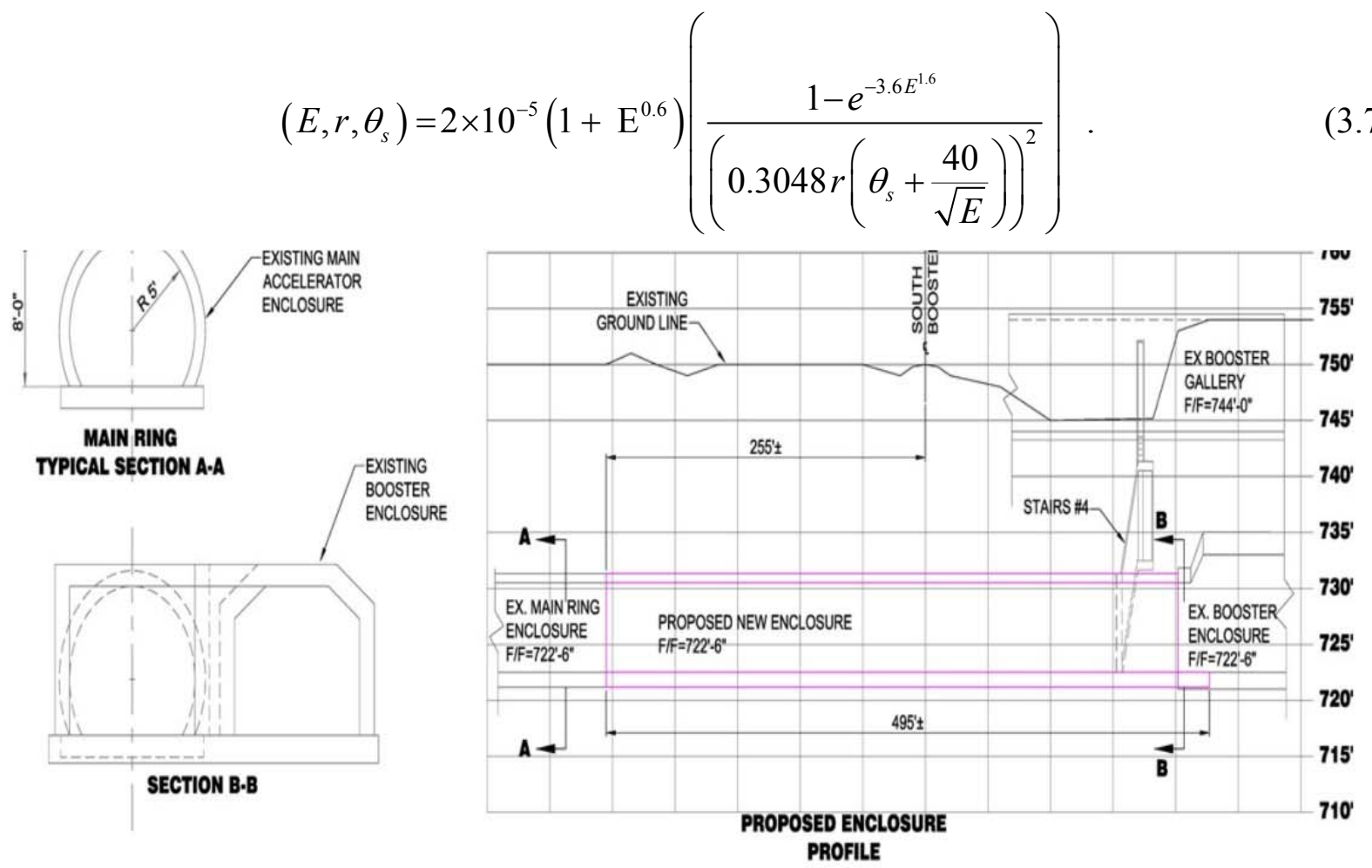

Figure 3.47: $1 \mathrm{GeV}$ transport line to the Booster accelerator

For neutron energies below $100 \mathrm{MeV}$, the attenuation length in concrete is significantly shorter than that for the neutrons considered in higher beam energy based assessments. For example, for high energy shielding problems, 3 feet of concrete provides a reduction factor of 10 in radiation dose rate. The mean free path of low energy neutrons relative to the high energy asymptote has been parameterized [86]:

$$
\frac{\lambda_{L E}}{\lambda_{H E}}=1-0.8 e^{-5^{*} E}
$$

The reduction in radiation dose rate as a function of energy ( $\mathrm{GeV}$ and concrete shield thickness (feet) is:

$$
A\left(E, T_{\text {conc }}\right)=10^{\frac{-T_{\text {conc }} /\left(1-0.8 e^{-5 E}\right)}{3}}
$$

In the dose rate calculations the peak neutron energy, $E(\mathrm{GeV})$, is taken to be equal to the beam energy. This simplification is conservative in that the actual neutron energies are necessarily lower and hence lead to better attenuation provided by the concrete shielding than indicated by Eq. (3.9). In addition, the dose equivalent per neutron conversion factor is taken as a constant value of $40 \mathrm{fSv} / \mathrm{n}$ over the full range of the neutron spectrum. Thus the resulting shielding calculations are implicitly conservative.

Radiation shielding required to limit radiation dose rates to $0.05 \mathrm{mrem} / \mathrm{hr}$ for a $1 \mathrm{GeV}$ linac and beam transport line assuming various beam levels of beam loss is shown in Figure 3.48. The shielding requirement varies with beam energy with the assumed maximum beam power loss for 134 
normal and accident conditions. The choice of shielding thickness will take into account a number of factors including the confidence level given to the Safety Analysis including consideration of the projected loss mechanisms and the machine protection system.

An active protection system, the Total Loss Monitor (TLM), currently under development, could be used to guarantee the limitation of any given beam power loss. The use of a TLM system could help to fix the level of beam power loss, and as a consequence, fix the amount of radiation shielding required.

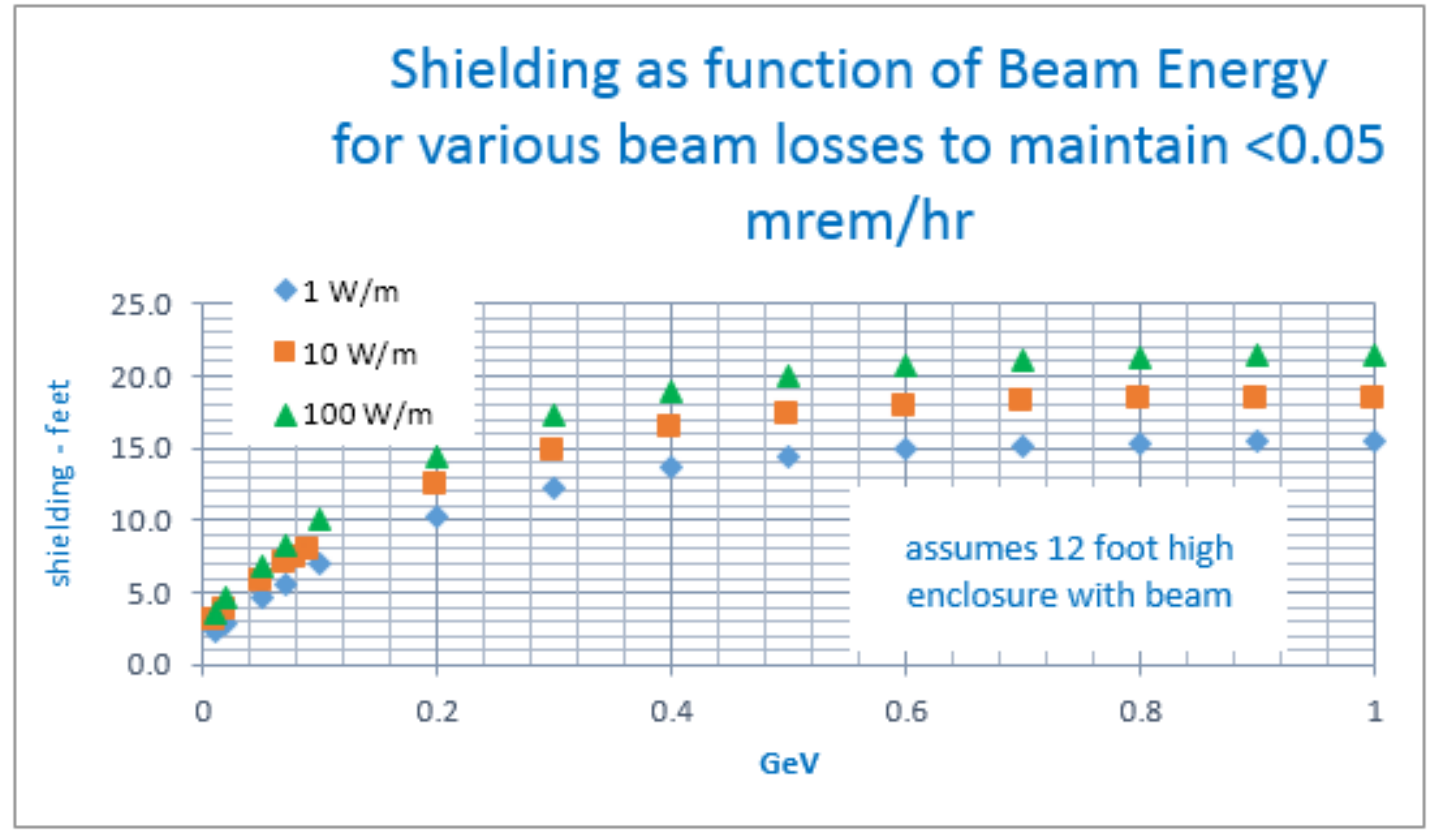

Figure 3.48: Radiation shielding requirements as a function of beam energy and beam power loss for a PIP-II beam enclosure

The TLM is an argon/ $\mathrm{CO}_{2}(80 \% / 20 \%)$ gas filled ion chamber of variable length with an applied bias voltage. Beam loss in the vicinity of the ion chamber produces a charge whose magnitude is proportional to the amount of beam loss. The TLM response to an $8 \mathrm{GeV}$ proton beam loss made under controlled conditions measured over a wide range of bias voltage and over two decades of beam intensity has been determined as shown in Figure 3.49. The response has been shown to be independent of the TLM length. At the nominal bias of 800 volts, the TLM response to $8 \mathrm{GeV}$ proton beam loss is about $3 \mathrm{nC} / \mathrm{E} 10$ protons. Preliminary scaling laws, to be verified in further TLM development work, can be used to predict TLM response at other energies.

The response can be scaled to beam energy down to $1 \mathrm{GeV}$ by the relationship:

$$
\frac{3 \mathrm{nC}}{10^{10} \text { protons }} \times\left(\frac{E}{8 \mathrm{GeV}}\right)^{0.8}
$$

The response to beam energy below $1 \mathrm{GeV}$ remains to be determined.

A feature of the TLM system is that an interlock trip level can be established to limit beam loss to $1 \mathrm{~W} / \mathrm{m}$ or virtually any beam power loss. A TLM, as presently conceived, does not 
distinguish between distributed losses and single point beam losses. The process to set TLM trip levels consists of two steps: (1) establish the total charge to be collected for a distributed loss, e.g., $1 \mathrm{~W} / \mathrm{m},(2)$ evaluate the shielding considering that the total charge is deposited at any single location. If the shielding is sufficient for the maximum charge collection rate at any location, then the TLM can effectively limit both the distributed beam loss and worst case single point beam loss.

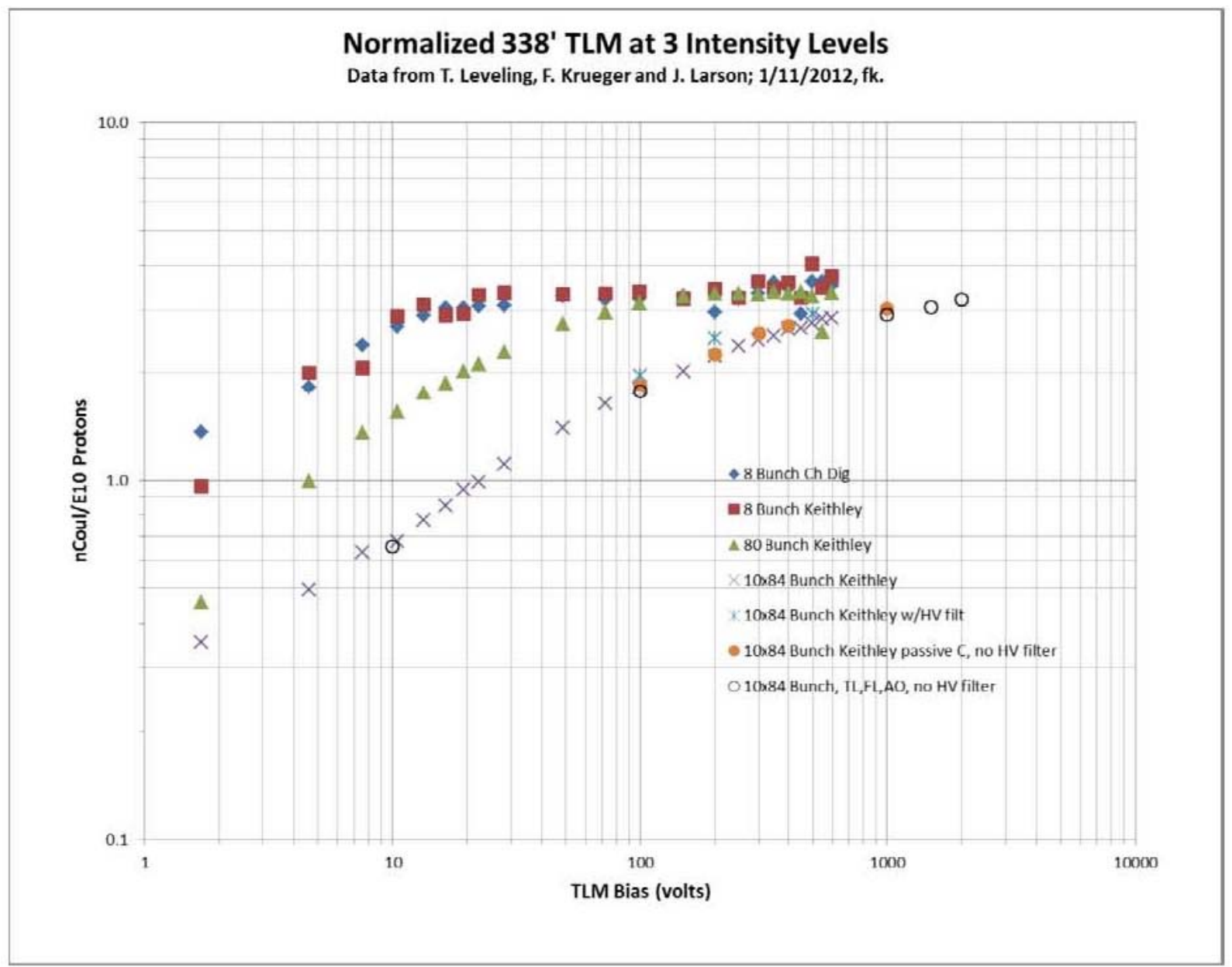

Figure 3.49: Response of 338 foot (103 meter) TLM as a function of applied bias voltage over 2 decades of beam intensity

Based upon preliminary TLM work, it should be possible to limit PIP-II beam loss with a TLM system beginning at the HWR cryomodule and continuing through the entire accelerator and the beam transport chain.

\section{Residual Activation of accelerator components and structures}

Residual radiation levels in beam transport lines and accelerators due to operational beam losses must be controlled in order to conduct maintenance activities while keeping personnel radiation exposure as low as reasonably achievable (ALARA). For 1.6-3 MW beam power, 136 
small fractions of $1 \%$ loss would result in very high residual radiation levels which would render beam enclosure access difficult and maintenance at loss points extraordinarily difficult. A sensitive machine protection system, which inhibits the beam operation when significant losses are present, is required to allow access and maintenance activities as historically enjoyed at Fermilab.

For design purposes, a loss rate of 3 to $10 \mathrm{~W} / \mathrm{m}$ results in a dose rate of about $100 \mathrm{mR} / \mathrm{hr}$ at one foot from beam line components such as magnets and accelerating cavities following a 30 day irradiation period and 1 day of cool down. A loss of 0.25 watts/meter results in a dose rate of about $100 \mathrm{mR} / \mathrm{hr}$ at one foot from low mass components such as beam pipes for the same irradiation/cooling period. Radiation levels considered acceptable are typically at least a factor of 5 less than these levels. For example, for a typical magnet beam loss location at $2 \mathrm{~W} / \mathrm{m}$, the fractional beam power loss is $1 \mathrm{ppm}$. A sensitive machine protection system will be required to quickly identify and suspend operation in the event such losses occur.

In the Safety Analysis, projected normal losses due to intrabeam scattering and other loss mechanisms are below $0.1 \mathrm{~W} / \mathrm{m}$. The machine protection system as presently conceived should limit beam loss to $<1 \mathrm{~W} / \mathrm{m}$. Consequently, residual activation of the accelerator, beam line components, and tunnel structures should be comparable to or less than levels tolerated in existing and previous machines. While the machine protection system would serve to protect the accelerator and beam line components, the TLM system would serve in a parallel role as a personnel safety system to limit residual activation of accelerator components.

\section{Air activation}

Air activation must also be characterized for the projected PIP-II operations. Based upon the anticipated losses described in the safety analysis, the combination of anticipated normal beam loss and the machine protection system should serve to limit the total beam loss levels at or below those produced at existing facilities. Based upon projected losses from the Safety Analysis, no significant air activation is anticipated. While the machine protection system would serve to protect the accelerator and beam line components, the TLM system would serve in a parallel role as a personnel safety system to limit air activation within accelerator enclosures.

\section{Water activation}

The site chosen for the new PIP-II accelerator and beam line enclosures is inside the former Tevatron ring. In order to evaluate surface and ground water activation, a geological survey (core borings) will be required to understand ground water migration rates at this site since no data presently exists. An estimate of surface and ground water activation is necessary in order to ensure compliance with regulatory requirements for surface and ground water. However, based upon losses projected by the Safety Analysis, no significant surface water or ground water activation is anticipated. The machine protection system would serve to limit the total beam loss that would also determine the level of surface water and ground water activation. The TLM system would serve a parallel, redundant role to also limit surface and ground water activation.

\section{Radioactive surface contamination}

Radioactive surface contamination results coincidentally with the activation of accelerator and 
beam line components. Maintenance activities are rendered more complicated when radioactive surface contamination is present due to prescriptions for the use of personnel protective equipment including coveralls, gloves, shoe covers, and other protective measures. It is possible in megawatt beam power machines to produce very significant levels radioactive surface contamination at beam loss locations. However, as indicated in the Safety Analysis, the nominal beam power losses are expected to be approximately $0.1 \mathrm{~W} / \mathrm{m}$, about a factor of 100 below the beam power loss required to produce the onset of measurable radioactive surface contamination. Consequently, radioactive surface contamination on accelerator, beam line components, and tunnel structures should be comparable to or less than levels tolerated in the existing and previous machines.

\section{Lifetime of machine components}

Based upon the level of beam loss projected by the Safety Analysis and also upon experience with existing accelerator and beam line facilities, machine component lifetimes should, in general, be on the order of many decades. 


\subsection{Machine Protection System}

The PIP-II linac will accelerate $2 \mathrm{~mA}$ beam current with $1.1 \%$ duty factor which results in an average beam current of $22 \mu \mathrm{A}$. After a planned upgrade to $\mathrm{CW}$ operation, the total beam current will be greater than in any, present HEP hadron linac. A robust Machine Protection System (MPS) will protect the linac components from direct beam induced damage and excessive radiation damage. The main goals of this MPS are as follows:

- Protect the accelerator from beam induced damage,

- Manage and monitor the beam intensity,

- Safely switch the beam off in the case of failures,

- Determine the operational readiness of the machine,

- Manage and display MPS alarms,

- Provide a comprehensive overview of the machine status,

- Provide high availability,

- Provide fail safe operation where possible,

- Provide post mortem analysis.

Several signals from devices or systems will be monitored and utilized as actuators to inhibit the beam at various stages of the accelerator. The main actuator for the beam is the ion source power supply itself. In addition, the LEBT/MEBT choppers' power supplies, the Radio Frequency Quadrupole (RFQ) amplifier, cavity power amplifiers, beam stops, and gate valves will act as additional control devices. A comprehensive overview of the entire machine will be obtained by careful monitoring all relevant inputs from machine diagnostics and critical systems affecting safe or fail safe operation.

The protection system model is based on experience gained from commissioning and operating the SNS accelerator. Its peak current specifications are about 20 times higher than the PIP-II peak beam current specification, and its copper to SC cavity transition occurs at $187 \mathrm{MeV}$. Above $200 \mathrm{MeV}$ the PIP-II MPS hardware design and placement can be modeled after the SNS system. The PIP-II MPS system will not need response times as stringent as the SNS because of lower peak currents. The challenge for the PIP-II MPS comes from the low energy cryomodule protection $(2.1 \mathrm{MeV}-150 \mathrm{MeV})$ where beam losses have difficulty penetrating the cryomodule and beam pipe.

\subsubsection{MPS Configuration}

The MPS will be considered to be the collection of all subsystems involved in the monitoring and safe delivery of beam to the dump and not limited to any particular subsystem or diagnostic device. It has connections to several external devices and sub-systems. Figure 3.50 shows a conceptual overview diagram of the MPS. The top layer comprises signal providers such as beam loss monitors, beam position monitors, magnet power supplies etc. Systems at this level send alarms or status information to the MPS logic subsystems (permit system) which issues a permit based on the comprehensive overview of all inputs and requests. Only simple digital signals (e.g. on-off, OK-alarm) are transmitted. All devices or subsystems that are determined to be pertinent to protecting the machine or necessary for machine configuration are included. The permit system layer of the MPS will be FPGA based and is thus fully programmable and handles 
complex logic tasks. The logic here will be designed to ensure safe operating conditions by monitoring operational input, chopper performance, the status of critical devices and by imposing limits on the beam power. The final layer of the system shows the main actuators. This will comprise all points where the MPS logic may act on the operation of the machine to prevent beam from being produced or transported.

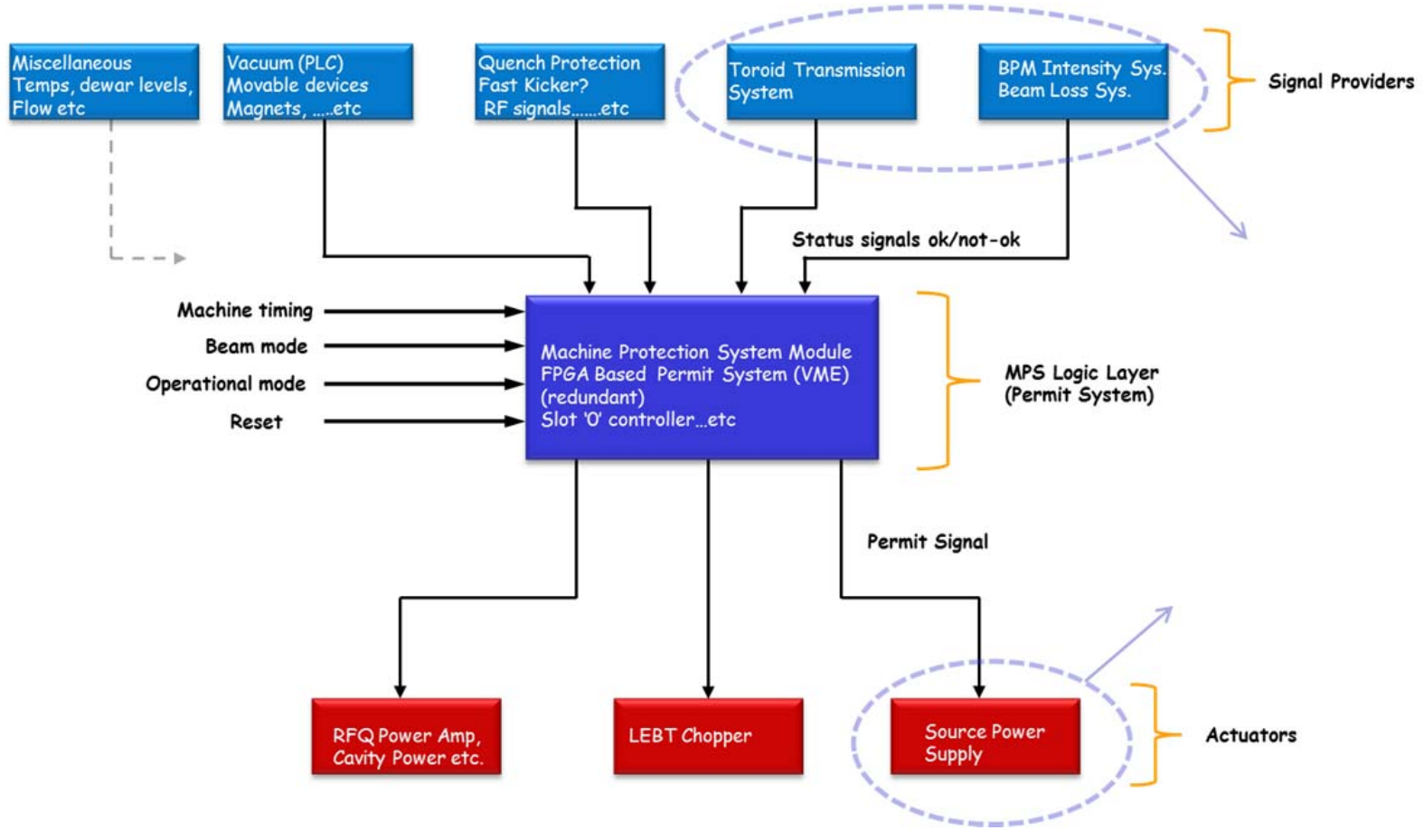

Figure 3.50: MPS Conceptual Layout

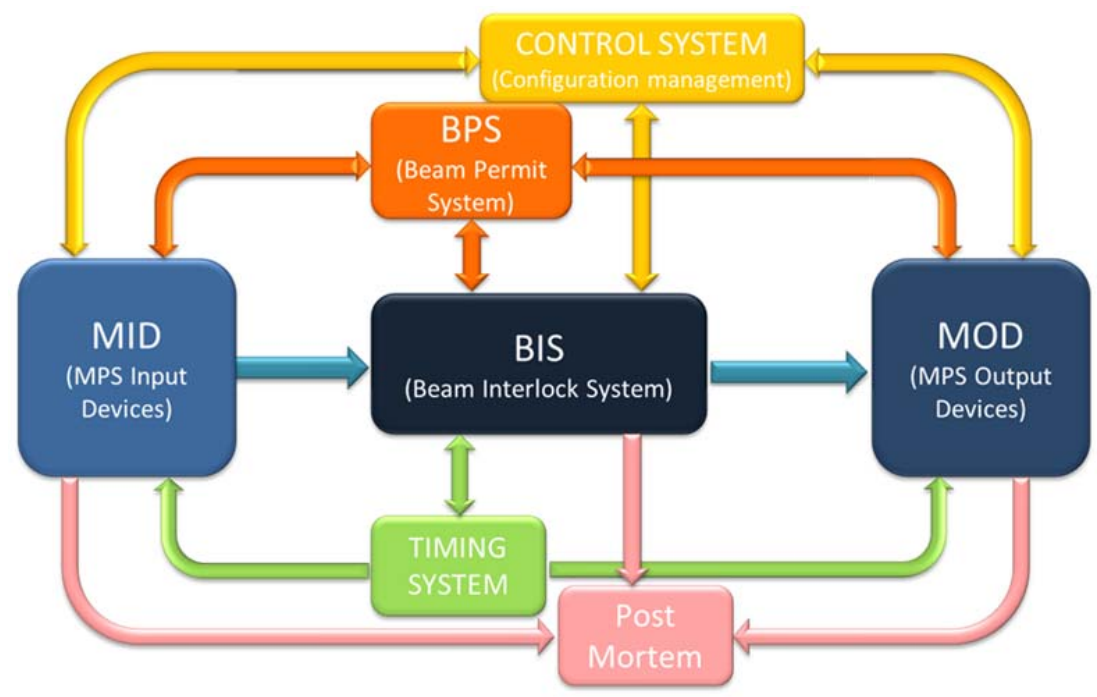

Figure 3.51: Conceptual MPS layout integrated with control system

The entire protection system interfaces with the accelerator control system and machine 
timing system for configuration management, timing and post mortem analysis as shown in Figure 3.51. The operational modes, operational logic, reaction time and complexity of inputs will differ based on the machine configuration and damage potential at various stages of the accelerator complex.

\subsubsection{Protection System R\&D}

Protecting the superconducting cavities from low energy protons losses where the particle energies are too low to produce significant detectable radiation will be a major part of the developmental work needed to effectively inject beam without quenches. To achieve this we will need to research sensitive means for measuring these losses and develop an effective feedback for machine protection. In addition we plan to achieve the following goals as a result of designing, constructing and operating the PXIE MPS:

- Understand and verify acceptable loss rates in the room temperature sections,

- Develop a strategy to monitor chopped beam from the MEBT,

- Estimate particle shielding effect of superconducting cavities and cryomodules,

- Develop effective algorithms for the FPGA based logic system,

- Demonstrate effective integration with controls/instrumentation and all subsystems,

- Understand dark current effects as it relates to protection issues.

In order to protect the accelerator from damage as the beam transitions from the room temperature sections of the machine to the superconducting sections, some specialized instrumentation may be developed at PXIE. Developing an effective algorithm to monitor the beam position as a feedback to machine protection will be of interest for both PXIE as well as PIP-II. 


\section{Siting and Conventional Facilities}

\subsection{Linac Siting}

The Linac site is in close proximity to the Booster, in the Tevatron infield (see Figure 1.1). This location affords direct access to existing electrical, water, and cryogenic infrastructure. It has the added benefit of minimizing the impact to existing wetlands. The proximity of the linac to the Booster is constrained by various existing utilities and buildings along Main Ring Road.

The linac gallery is of similar length as the underground linac enclosure, housing the utilities and support equipment to operate the RF power systems, magnets, vacuum, and controls. It is located adjacent to the linac enclosure.

The linac enclosure is sited at the same elevation as the Booster and the Tevatron tunnel, and the transfer line crosses through the Tevatron tunnel which holds the transfer line directed to the $120 \mathrm{GeV}$ Switchyard. As the Switchyard program is assumed to continue, the linac-to-Booster transfer line must be integrated with the existing $120 \mathrm{GeV}$ beam line. The Booster injection is located in the Long 11 straight section. Using this straight section results in minimal displacements of tunnel equipment and also reduces the interference with electrical utilities.

Surface construction includes buildings, road and parking relocation, and additional roadways and access from the Fermilab Central Campus. Underground construction includes the linac enclosure, the transfer line enclosure, and a beam dump enclosure.

While the working cryogenics arrangement does not necessarily require new building space, a possible siting has been identified at the downstream end of the linac, somewhat further into the Tevatron infield.

\subsection{Technical Requirements and Scope}

The linac enclosure is sized to accommodate the length of a $0.8 \mathrm{GeV} \operatorname{linac}^{8}(\sim 220 \mathrm{~m}, 721 \mathrm{ft})$. The enclosure has to provide adequate space for the linac hardware, penetrations for utilities (power, water, cryogens) and cabling, as well as for equipment installation and maintenance. It also has to include access points for moving equipment in and out of the enclosure. The linac gallery is slightly shorter than the enclosure $(\sim 210 \mathrm{~m}, 689 \mathrm{ft})$.

The current concept for the cryogenics configuration utilizes space in the A-0 high-bay building to host the required compressors and refrigerators. If for some reason (e.g. extended Muon Campus operations) this is not achievable, a new cryogenics building will be constructed.

The existing cryo pipes that run from A- 0 to the MC-1 Building will cross the planned transfer tunnel. See attached Plan View in Figure 4.1 and utility line type legend in Figure 4.2. All existing utilities located above the planned beam enclosure will need to be either supported during excavation; or, shutdown, removed, and reinstalled after the beam enclosure construction.

\footnotetext{
${ }^{8}$ This length also includes additional space for four HB650 cryomodules which can be installed later as an upgrade of the linac energy. In described here PIP-II project this space will be used for the fast beam switch (see Section 2.2.3).
}

142 


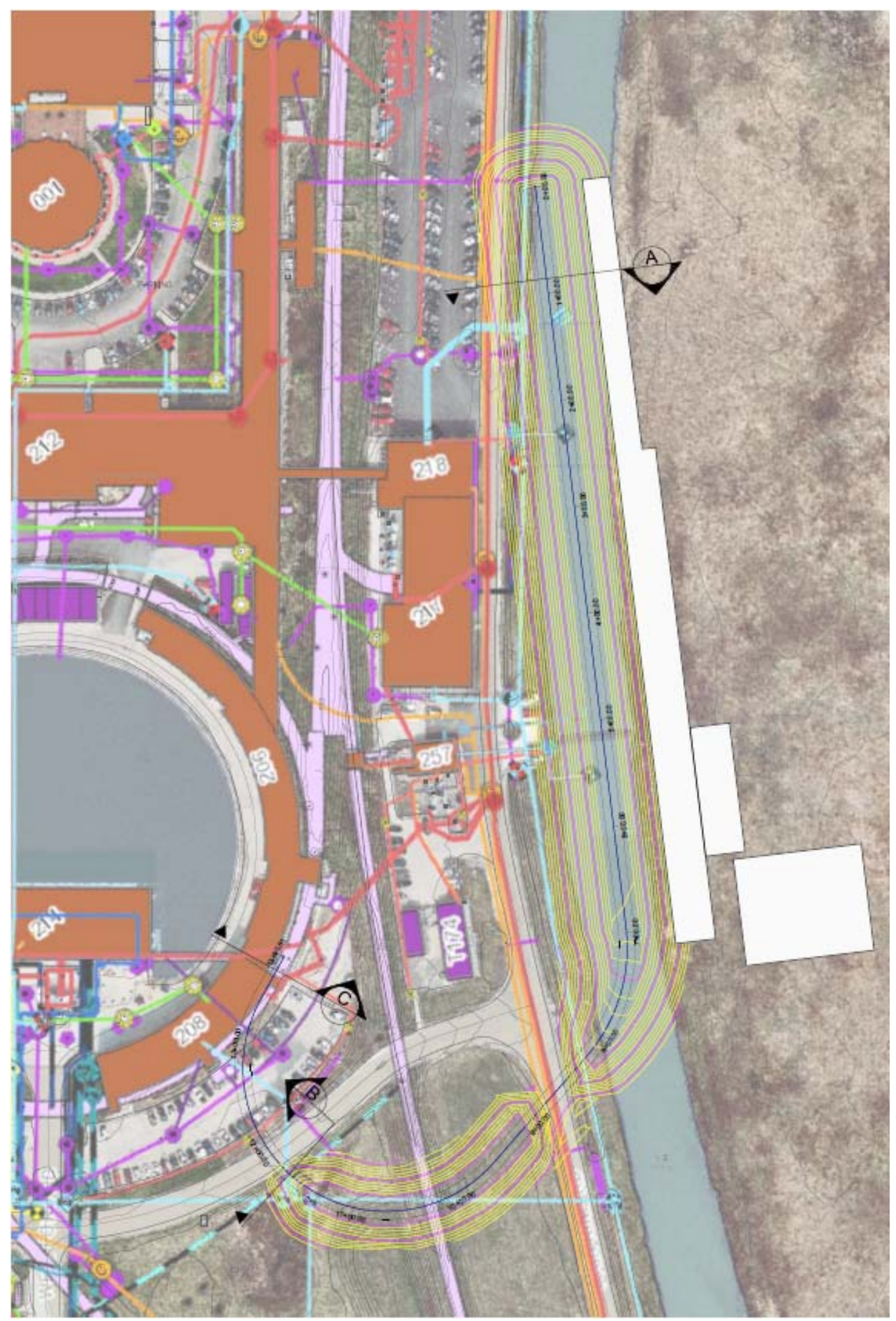

Figure 4.1: Plan view in vicinity of PIP-II linac.

143 


\begin{tabular}{|c|c|c|c|c|c|}
\hline \multicolumn{6}{|c|}{ Fermi Map Legend } \\
\hline $\begin{array}{l}\text { Facilities } \\
\text { Building-Trailer } \\
\text { Property Type } \\
\begin{array}{|l}\text { Bulding } \\
\square \text { Trailer } \\
\square \text { Endosure }\end{array}\end{array}$ & 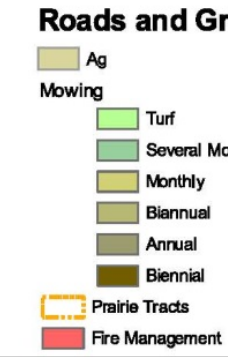 & & & $\begin{array}{l}\text { Land Planning } \\
\diamond \text { Archaeology } \\
- \text { Soil Borings } \\
\quad \text { Monitoring Wells } \\
\square \text { Easements } \\
\square \text { Regulated Area } \\
\square \text { Regulated Area 50ft buffer } \\
\square \text { Prohibited Excavetion Waiver Areas }\end{array}$ & $\begin{array}{l}\text { Basemap } \\
\begin{array}{l}\text { Roads } \\
\square \text { Parking Las } \\
\square \text { Streams } \\
\square \text { Ponds } \\
\square \text { Formi Boundary }\end{array}\end{array}$ \\
\hline 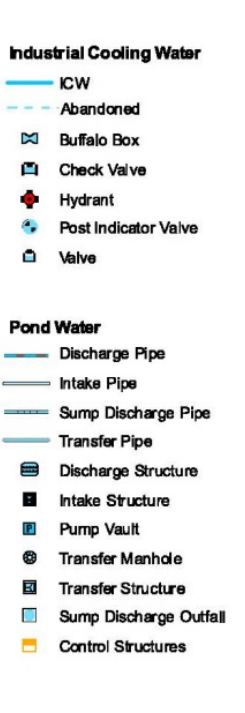 & 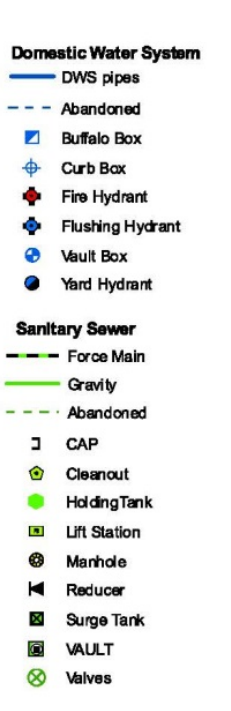 & 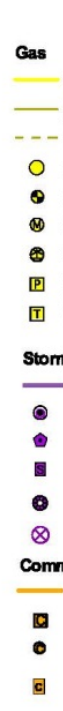 & $\begin{array}{l}\text { Utilities } \\
\text { Natural Gas Pipes } \\
\text { - Non-FNAL Gas } \\
\text { Gas Abandoned } \\
\text { Gas Manhole } \\
\text { Gas Valve } \\
\text { Meter } \\
\text { Press. Reducing Statio } \\
\text { Pressure Regulator } \\
\text { Test Station } \\
\text { m Drain } \\
\text { - Stom Pipe } \\
\text { Catchbasin } \\
\text { Cleanout } \\
\text { Lst Station } \\
\text { Manhole } \\
\text { Valve } \\
\text { munication } \\
\text { - commurication } \\
\text { Pedestal } \\
\text { Manhde } \\
\text { Handhole }\end{array}$ & 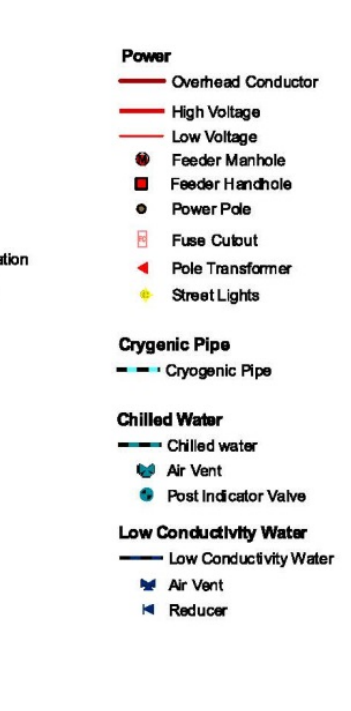 & $\begin{array}{ll}\text { Field Tile } \\
\longrightarrow & \text { CLAY } \\
\longrightarrow & \text { CONCRETE } \\
\longrightarrow & \text { POLYETHYLNE } \\
& \text { nvesigation Trench } \\
& \text { Data Points } \\
- & \text { Une Points } \\
\text { a } & \text { Riser } \\
\times & \text { Abandoned } \\
\text { Q } & \text { Outlet } \\
* & \text { Plug }\end{array}$ \\
\hline
\end{tabular}

Figure 4.2: Linetype Legend for Figure 4.4.

The linac beam elevation is chosen to match the Booster elevation. A concept cross section of the enclosure can be seen in Figure 3.42. It is surrounded by $7.5 \mathrm{~m}(24.5 \mathrm{ft})$ of passive earth shielding to allow unlimited occupancy of the linac gallery and surrounding areas (see Section 3.7). In order to obtain $24.5^{\prime}$ of shielding, the berm elevation over the Linac needs to be at 763.5'. In comparison, the top of the main ring berm is at an elevation of approximately 751 '. Additional steel will be needed at both crossings with existing roads and as the beam gets closer to the Booster tunnel.

The transfer line brings the beam from the linac end to the Booster. The enclosure has a similar cross section to the existing Tevatron tunnel and is $\sim 225 \mathrm{~m}$ (737 ft) in length. The transport line includes two arcs connected by $\sim 27 \mathrm{~m}$ straight line. The radius of arcs is $50.7 \mathrm{~m}$ $(166 \mathrm{ft})$ to minimize magnetic stripping for the $800 \mathrm{MeV} \mathrm{H}^{-}$beam and to have sufficient space for installation of beam instrumentation and if required the beam collimators. The total bend in the line is approximately $217^{\circ}$. The linac dump is connected to the linac enclosure by a short line and handles the full linac beam power of $20 \mathrm{~kW}$.

The Linac and transfer beam enclosures are assumed to have a floor elevation of 722.5', 
matching the main ring and booster tunnel floor elevations. Figures $4.3-4.5$ present three cross sections of the beam enclosure. Section A shows the Linac beam enclosure at Station 0+86.12. Section B shows the transfer tunnel enclosure at the utility crossing with existing ICW and cryo pipes at Station $11+67.87$. Section $C$ shows the transfer tunnel as the beam connects to the existing Booster tunnel. This cross section shows the above-grade section of Booster Tower Southeast.

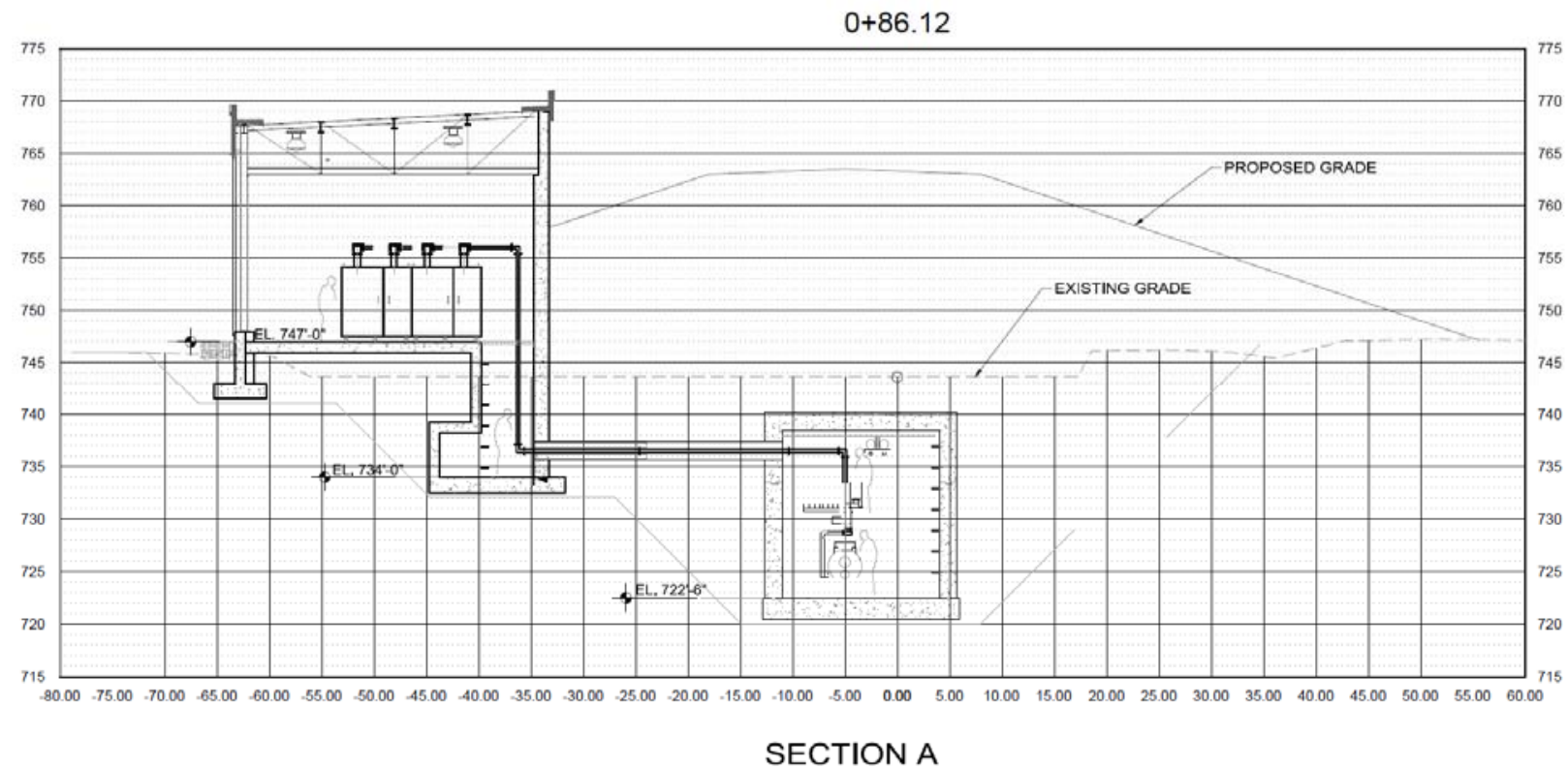

Figure 4.3: Cross sections of the beam enclosure Station 0+86.12.

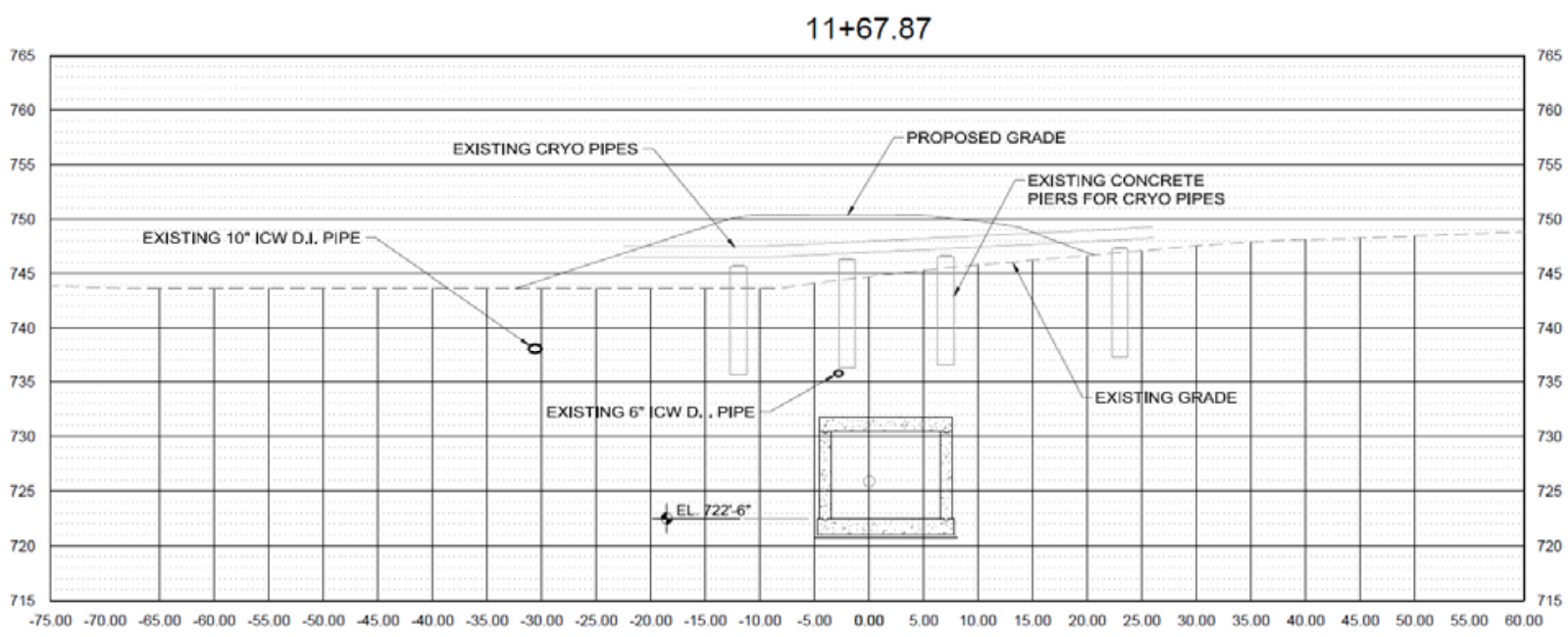

SECTION B

Figure 4.4: Cross sections of the transfer tunnel enclosure at the utility crossing with existing ICW and cryo pipes at Station $11+67.87$. 


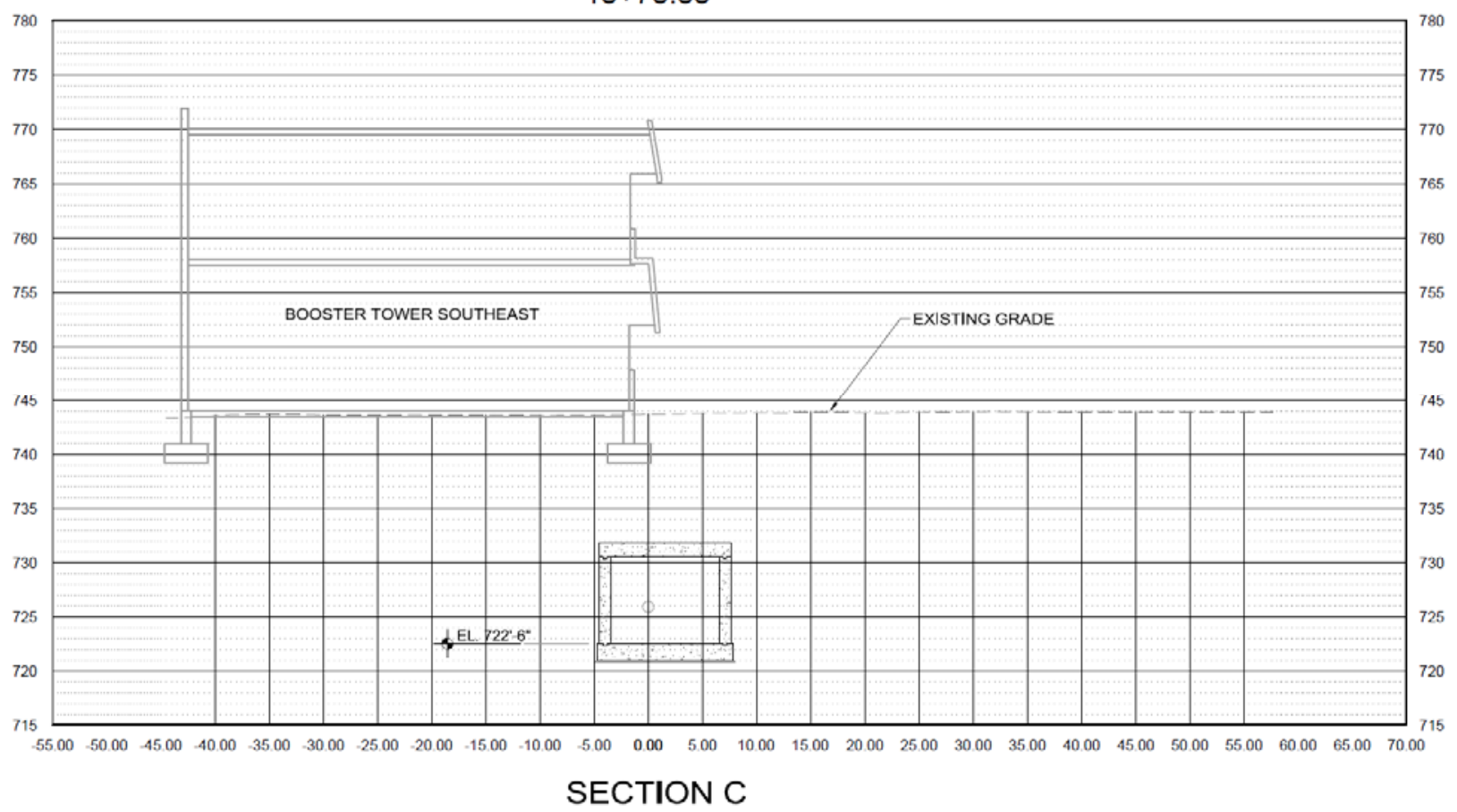

Figure 4.5: Cross sections of the transfer tunnel at location where the beam connects to the existing Booster tunnel.

Also impacted will be the Booster Tower Southeast building. The PIP-II transfer tunnel will approach the existing building's foundation in order to tie into the existing Booster tunnel. A portion of the building may need to be removed and rebuilt in order to excavate and install the transfer tunnel.

The Booster Tower Southeast parking lot will also be impacted. If the parking lot needs to remain, then additional steel shielding will need to be installed over the tunnel. Otherwise, the parking lot could be turned into a grassy berm similar to what was done for the Booster Tower Southwest; however, vehicular access to existing electrical equipment in the northeast corner of the parking lot will need to be provided for maintenance of the electrical equipment. Also, the construction of a berm will modify the existing storm water drainage in this area and will require additional storm structures and conveyance systems in order to properly drain the northeast area of the lot.

\subsection{Conventional Facilities}

This section outlines the conventional facilities required to house and support the SC Linac, its beam dump and the Linac-to-Booster transfer line. Civil construction for the facility includes all below-grade beam-line enclosures. All above-grade buildings, roads, parking, utilities and services to accommodate the equipment for the operation of the facility on the Fermilab site are also included.

Construction of the below-grade linac(s) and beam transport lines as well as the above-grade service buildings are similar to previously utilized and proven construction methods previously 
executed at Fermilab. Construction of all below-grade enclosures consists of conventional opencut type construction techniques. The architectural style of the new buildings reflects, and is harmonious with, the existing buildings. Currently, the layout has been optimized based on accelerator requirements. Future layouts will consider existing topography, sustainability, watersheds, vegetation, natural habitat, and wetlands. All the aspects will be thoroughly addressed in the Environmental Assessment for this project.

\section{Site Construction}

1) Site work

a) Site Drainage will be controlled by ditches and culverts, preserving the existing watershed characteristics both during construction and subsequent operation.

b) Road Construction will provide permanent access to all service buildings.

c) Landscaping includes the restoration of disturbed areas. Construction yards and stockpile areas will be removed after completion of the construction phase of the project. All disturbed areas will be returned to a natural state or landscaped in a similar manner as found at other Fermilab experimental sites. Erosion control will be maintained during all phases of construction.

d) Wetlands Mitigation includes the avoidance or minimization of adverse impacts to wetlands in the project area. Environmental consultants would delineate wetlands, and a Clean Water Act permit application prepared for submittal to U.S. Army Corps of Engineers for impacts that cannot be completely avoided. Compensatory mitigation would be provided according to terms and conditions of the permit. This may be in the form of purchased wetland bank credits, restoration or enhancement of existing wetlands on site, or creation of new wetland areas. The permit would dictate the amount and type of mitigation, which must be in place prior to the initiation of construction. A Floodplain/Wetland Assessment pursuant to 10 CFR 1022 would be incorporated into the Environmental Assessment.

2) Utilities

The following utilities are required to support the operation of the facility. The list incorporates current assumptions and will require further refinement as the design process progresses.

a) Electrical Power includes new duct banks and utilization of existing duct banks from two sources including Kautz Road Substation (KRS) and Master Substation (MSS). Separate high-voltage feeders with backup will be provided for conventional, machine and cryogenic power.

b) Communications include new duct banks tied into the existing communication network along Main Ring Road.

c) Chilled Water (CHW \& CHWR) for machine and building cooling will be supplied via new supply and return lines from the existing Central Utility Building (CUB).

d) Low Conductivity Water (LCW) for machine cooling will be supplied via new supply and make-up water from the existing Main Injector ring LCW system.

e) Industrial Cooling Water (ICW) for fire protection will be supplied via new supply and return lines along Main Ring Road.

f) Domestic Water Supply (DWS) for potable water and facilities will be supplied via new 
supply line from the existing site wide DWS system.

g) Sanitary Sewer (SAN) for facilities will be supplied via new sewer main and lift station from to the existing site wide sanitary sewer system.

h) Natural Gas (NGS) for building heating will be supplied via new supply lines from the existing site wide NGS system.

3) Facilities Construction

Conventional facilities will be constructed with future upgrade capabilities considered in the initial design phase. Equipment galleries, enclosures and surface buildings will be designed to accommodate future expansion of the technical components of the facility. The major elements for the conventional facilities are as follows:

a) Below-Grade Construction

0 to $0.8-\mathrm{GeV}$ SC Linac Enclosure

Linac-to-Booster Line Enclosures

b) Above-Grade Construction

0 to $1-\mathrm{GeV}$ Linac Gallery

Center Service Building

\subsection{Site Power Requirements}

An estimate of site power requirements for the linac operating in the pulsed mode and the beam transfer line to the Booster is given in Table 4.1.

The following items were included into the power estimate:

- RFQ and MEBT RF: includes RF sources powering RFQ and MEBT rebunching cavities operating in $\mathrm{CW}$ regime.

- SC Linac RF: includes RF sources powering SC cavities to accelerate $2 \mathrm{~mA}$ of beam to $0.8 \mathrm{GeV}$ with RF duty factor of $12 \%$. Also included are LLRF, protection circuits and RF controls.

- Magnets: Includes all power supplies for quads, solenoids, dipoles and trim magnets.

- Cryogenic Systems: Based on the CHL upgrade described in Section 3.4.

- LCW (Low Conductivity Water): The primary load is cooling of the RF sources and power supplies for dipoles.

- $\quad$ HVAC (heating, ventilation and air-conditioning): The primary loads are RF power not removed by the LCW system and the removal of heat from equipment galleries.

- Conventional Systems: Power required for other linac/beamline components (vacuum pumps), and for occupied spaces.

The total PIP-II SC linac power is $\sim 6.2 \mathrm{MW}$. It supports its operation in the pulsed regime. 
Table 4.1: Site power estimates.

\begin{tabular}{l|c}
\hline \hline System & Wall-Plug Power (kW) \\
\hline RFQ and MEBT RF & 320 \\
\hline SC linac RF & 1100 \\
\hline Magnets (quads, solenoids, dipoles) & 300 \\
\hline Cryogenic Systems & 3250 \\
\hline LCW & 200 \\
\hline Chilled water & 500 \\
\hline HVAC & 200 \\
\hline Conventional Systems & 300 \\
\hline Total & 6170 \\
\hline
\end{tabular}




\section{References}

[1] Building for Discovery: Strategic Plan for U.S. Particle Physics in the Global Context, http://science.energy.gov/ /media/hep/hepap/pdf/May\%202014/FINAL_P5_Report_05301 $\underline{4 . p d f}$

[2] LBNE Conceptual Design Report Volume 2: The Beamline at the Near Site, October 2012, http://lbne2-docdb.fnal.gov/cgi-bin/RetrieveFile?docid=4317;filename=CDR-beamvolume-101812-reduced.pdf

[3] Proton Improvement Plan website, http://www-ad.fnal.gov/proton/PIP/PIP index.html

[4] Project X: Accelerator Reference Design, S. D. Holmes (editor), http://arxiv.org/abs/1306.5022

[5] Project X: Physics Opportunities, A.S. Kronfeld, R.S. Tschirhart (editors), http://arxiv.org/abs/1306.5009

[6] Project X: Broader Impacts, David Asner/Pacific Northwest National Laboratory, D. Asner, P. Bhat, S. Henderson, R Plunkett (editors), http://arxiv.org/abs/1306.5024

[7] McGinnis, D (editor), "Accelerator Issues of Project X", http://projectx-docdb.fnal.gov/cgi-bin/ShowDocument?docid=143

[8] Derwent, P (editor), "Project X Initial Configuration Document V1.1", http://projectx-docdb.fnal.gov/cgi-bin/ShowDocument?docid $=83$

[9] Derwent, P (editor), "Project X Initial Configuration Document-2", http://projectx-docdb.fnal.gov/cgi-bin/ShowDocument?docid=230

[10] SNOWMASS WORKING GROUP REPORTS, http://www.snowmass2013.org/tiki-index.php

[11] G. E. Krafczyk, " Booster 20 Hz tests”, Beams-doc-4777, http://beamdocs.fnal.gov/AD-public/DocDB/ShowDocument?docid=4777

[12] M. Plum, et.al., "STATUS OF THE SNS RING POWER RAMP UP", Proceedings of EPAC08, p. 3560-3562.

[13] I. Kourbanis, et.al., "Progress Toward Doubling the Beam Power at Fermilab's Accelerator Complex", Proceedings of IPAC 2014 (FERMILAB-CONF-14-187-AD).

[14] J.N. Galayda, "The Linac Coherent Light Source-II Project,” IPAC2014, Dresden, June 15-20, TUOCA01, p.935.

[15] Project X Document 912: "Project X LEBT: Functional Requirement Specifications", http://projectx-docdb.fnal.gov/cgi-bin/ShowDocument?docid=912

[16] Ji, Q and Staples, J, Project X Document 897: "Project X H' Ion Source Acceptance Test and Future Plan", http://projectx-docdb.fnal.gov/cgi-bin/ShowDocument?docid=897

[17] K.R. Crandall et al. "RFQ Design Codes", Los Alamos Report LA-UR-96-1836 (1996, Revised, Dec. 7 2005)

[18] R. Duperrier. N. Pichoff and D. Uriot, "CEA Saclay Codes Review for High Intensities Linacs Computations", Lecture Notes in Computer Science Volume 2331, 2002, pp 411 418.

[19] Diebele, C and Aleksandrov A., "Experimental study of the SNS MEBT chopper 
performance", in Proc. of IPAC'10, Kyoto, Japan, 2010, MOPD063.

[20] Project X Document 938: "PXIE MEBT Functional Requirements Specification", http://projectx-docdb.fnal.gov/cgi-bin/ShowDocument?docid=938

[21] Project X Document 933: "PXIE MEBT quadrupoles specifications", http://projectxdocdb.fnal.gov/cgi-bin/ShowDocument?docid $=933$

[22] Project X Document 977: "PXIE MEBT kicker specifications", http://projectxdocdb.fnal.gov/cgi-bin/ShowDocument?docid $=977$

[23] Lebedev, V, et al, "Progress with PXIE MEBT chopper", in Proc. of IPAC'12, New Orleans, USA, 2012, WEPPD078.

[24] Project X Document 1071: "FRS for Bunching cavity for PXIE MEBT", http://projectxdocdb.fnal.gov/cgi-bin/ShowDocument?docid=1071

[25] Yakovlev, V, "SRF Linac Technology Development", Proceedings of the XXVI Linac Conference (LINAC12), Tel-Aviv, 2012.

[26] Ciovati, G, "Review of High Field Q Slope, Cavity Measurements", in Proc. SRF2009: 90 (2009).

[27] Project X Document 590: “Assumption about Q values in CW linac", http://projectxdocdb.fnal.gov/cgi-bin/ShowDocument?docid $=590$

[28] Reschke, D, "Analysis of the RF results of recent 9-cell cavities at DESY", TTC-Report 2009-01, 2009

[29] Brunner, O, et al, "Assessment of the basic parameters of the CERN Superconducting Proton Linac", PRSTAB 12, 070402, 2009

[30] Technical Specifications for High RRR Grade Niobium Sheet for Use in Superconducting Radio Frequency Cavities, Fermilab Specification: 5500.000-ES-371037 Rev B, Fermilab, Technical Division.

[31] Chase, B., Peterson, Th. and Yakovlev, V.,"A Strategy for Cavity Resonance Control in the Project X CW Linac,” 02.20.2011, Project X Document 827-v1, ProjectX Database, http://projectx-docdb.fnal.gov/cgi-bin/ShowDocument?docid=827.

[32] V. Lebedev, et.al. "Intrabeam stripping in $\mathrm{H}^{-}$linacs", Linac-2010, Tsukuba, Japan, Sep. 1217, 2010.

[33] A. Shishlo, J., et.al. "First Observation of Intrabeam Stripping of Negative Hydrogen in a Superconducting Linear Accelerator" Plum, Phys. Rev. Lett. 108, 114801 (2012).

[34] Krivosheev, O. and Mokhov, N. "Tolerable beam loss at High-Intensity Proton Machines", Fermilab-Conf-00/185, Aug. 2000.

[35] J.-P. Carneiro, "H- Stripping Equations and Application to the High Intensity Neutrino Source", Beams-doc-2740, http://beamdocs.fnal.gov/AD-public/DocDB/DocumentDatabase

[36] G.H. Gillespie, Phys. Rev. A 15, 563 (1977) and 16, 943 (1977), Nucl. Intr. \& Meth. B 2, 231 (1984) and B 10/11, 23 (1985).

[37] J. Lackey, et. al., "Operation and Performance of the New Fermilab Booster H- Injection System”, FERMILAB-CONF-07-248-AD. 
[38] M. Popovic, et. al., "A Proposed H- Injection System for the Fermilab Booster”, Beamsdoc 1784-v1, April 2005.

[39] M.S. Gulley et al., Phys. Rev. A 53, 3201 (1996).

[40] W. Chou, M. Kostin and Z. Tanga, "Stripping Efficiency and Lifetime of Carbon Foils," http://arxiv.org/abs/physics/0611157

[41] V. Lebedev and V. Shiltsev (Eds.), "Accelerator Physics at the Tevatron Collider", Springer, 2014.

[42] Yu. Tokpanov, V. Lebedev, W. Pellico, "MEASUREMENTS OF MAGNETIC PERMEABILITY OF SOFT STEEL AT HIGH FREQUENCIES”, Proceedings of IPAC2012, New Orleans, Louisiana, USA.

[43] James L. Crisp and Brian J. Fellenz, "Measured Longitudinal Beam Impedance of Booster Gradient Magnets", Fermilab-TM-2145, March 22, 2001.

[44] V. Lebedev and C. Bhat, "Booster Longitudinal Impedance," http://www-bdnew.fnal.gov/pxie/PIPIImeetings/BoosterLongImpedance.pdf

[45] W. Pellico, "From PIP to PIP-II," https://beamdocs.fnal.gov/AD/DocDB/0047/004700/002/Booster-PIPII-v1.pdf

[46] King-Yuen Ng, "Longitudinal Coupled Bunch Instability in the Fermilab Booster", FERMILAB-FN-0464; KEK-87-17.

[47] A. Burov and V. Lebedev, FERMILAB-TM-2492-AD (2011)

[48] A. Macridin et al., Phys. Rev. ST-AB, 14, 061003 (2011)

[49] A. Burov, Phys. Rev. ST-AB, 12, 044202 (2009)

[50] A. Burov, Phys. Rev. ST-AB, 17, 021007 (2014)

[51] A. Chao, "Physics of Collective Beam Instabilities in High Energy Accelerators", John Wiley \& Sons, Inc., Eq. (6.58), (1993)

[52] A. Macridin et al., FERMILAB-CONF-13-431-CD (2013).

[53] A. Macridin. Private communication

[54] A. Burov, FERMILAB-PUB-11-174-AD (2011)

[55] See Eq. (6.240), p. 360. in Ref. [51]

[56] J. Wei, "Transition Crossing", in "Handbook of Accelerator Physics and Engineering" (edited by A.W. Chao and M. Tigner), World Scientific, $2^{\text {nd }}$ Printing (2002).

[57] Project X Document 968: "Project X and PXIE Ion Source Functional Requirement Specifications", http://projectx-docdb.fnal.gov/cgi-bin/ShowDocument?docid=968

[58] http://www.dehnel.com/index.html

[59] Project X Document 1077: "PXIE LEBT Beam Transverse Emittance Station FRS", http://projectx-docdb.fnal.gov/cgi-bin/ShowDocument?docid=1077

[60] Project X Document 894: "Project X RFQ functional physics requirements", http://projectx-docdb.fnal.gov/cgi-bin/ShowDocument?docid=894

[61] Virostek, S, et al, "Design and analysis of the PXIE CW Radio-Frequency Quadrupole (RFQ)", in Proc. of IPAC2012, New Orleans, Louisiana, USA, 2012, THPPC034. 
[62] Ratti, A, et al, "The Design of a High Current, High Duty Factor RFQ for the SNS", in Proc. of EPAC '00, Vienna, Austria, 2000, pp. 495-497.

[63] Romanov, G, et al, "Project X RFQ EM Design”, in Proc. of IPAC'12, New Orleans, Louisiana, USA, 2012, THPPP064.

[64] Project X Document 933: "PXIE MEBT quadrupoles specifications", http://projectx-docdb.fnal.gov/cgi-bin/ShowDocument?docid=933

[65] Project X Document 977: "PXIE MEBT kicker specifications," http://projectx-docdb.fnal.gov/cgi-bin/ShowDocument?docid=977

[66] Project X Document 964: "Functional specifications for PXIE MEBT absorber", http://projectx-docdb.fnal.gov/cgi-bin/ShowDocument?docid=964

[67] A. Shemyakin and C. Baffes, High power density test of PXIE MEBT absorber prototype, Proc. of LINAC'14, August 31 - September 5, 2014, Geneva, Switzerland, THPP055.

[68] Project X Document 1071: "FRS for Bunching cavity for PXIE MEBT”, http://projectxdocdb.fnal.gov/cgi-bin/ShowDocument?docid=1071

[69] Romanov, G, et al, "CW room temperature re-buncher for the Project $\mathrm{X}$ front end" in Proc. of IPAC'12, New Orleans, USA, 2012, THPPP063

[70] Project X Document 1067: "Functional specifications for PXIE MEBT scrapers", http://projectx-docdb.fnal.gov/cgi-bin/ShowDocument?docid=1067

[71] Project X Document 1043: "Specifications for PXIE MEBT BPMs", http://projectx-docdb.fnal.gov/cgi-bin/ShowDocument?docid=1043

[72] ATLAS $72 \mathrm{MHz}$ energy upgrade cryomodule

[73] Kephart, R (editor), "Project X Functional Requirements Specification," http://projectx-docdb.fnal.gov/cgi-bin/ShowDocument?docid $=658$

[74] Schappert,W, et al, "Microphonics Control for Project X," PAC2011, New York, March 28, 2011, TUP086.

[75] V.Yakovlev, et al, "HOMs in the Project-X CW Linac," Int. Workshop on Higher-Order Mode Damping in Superconducting RF Cavities, Cornell University, October 11-13, 2010, http://www.Ins.cornell.edu/Events/HOM10/rsrc/LEPP/Events/HOM10/Agenda/MA3 Yako vlev.pdf

[76] S.-H. Kim, "HOM Experiences at the SNS SCL," SPL HOM workshop, CERN, June 2526, 2009. http://indico.cern.ch/event/57247/session/1/contribution/9/material/slides/1.pdf

[77] A. Grassellino, A. Romanenko, O. Melnychuk, Y. Trenikhina, A. Crawford, A. Rowe, M. Wong, D. Sergatskov, T. Khabiboulline, F. Barkov, 2013 Supercond. Sci. Technol. 26 102001 (Rapid Communication).

[78] A. Romanenko, A. Grassellino, A. C. Crawford, D. A. Sergatskov, and O. Melnychuk, Appl. Phys. Lett. 105, 234103 (2014).

[79] N.V. Mokhov, C. James, The MARS Code System User's Guide, http://www-ap.fnal.gov/MARS/

[80] N. V. Mokhov, S.I. Striganov, "MARS15 Overview", in Proc. of Hadronic Shower Simulation Workshop, Fermilab, AIP Conf. Proc. 896 (2007) pp. 50-60. 
[81] N. Mokhov, P. Aarnio, Y. Eidelman, K. Gudima, A. Konobeev, V. Pronskikh, I. Rakhno, S. Striganov, I. Tropin, "MARS15 code developments driven by the intensity frontier needs," Progress in Nuclear Science and Technology 4, 496 (2014).

[82] Foster, GW, Chou W, E. Malamud E (ed), "Proton Driver Study II", Chapter 16, FermilabTM-2169, (May 2002).

[83] P.Treitea, et al., Design, project execution, and commissioning of the $1.8 \mathrm{~K}$ superfluid helium refrigeration system for SRF cryomodule testing, ICEC/ICMC 2014 https://indico.cern.ch/event/244641/session/25/contribution/41.pdf

[84] Cahill, K, et al, "The Fermilab Accelerator Control System”, ICFA Beam Dyn.Newslett.47:106-124, 2008

[85] Patrick, J and Lackey, S, “Control Systems for Linac Test Facilities at Fermilab", Proc. Linac08, Victoria, BC, 2008

[86] Sullivan, AH, "A Guide to Radiation and Radioactivity Levels Near High Energy Particle Accelerators", Nuclear Technology Publishing, (1992). 